\title{
Laser-driven proton acceleration and detection at high repetition rate
}

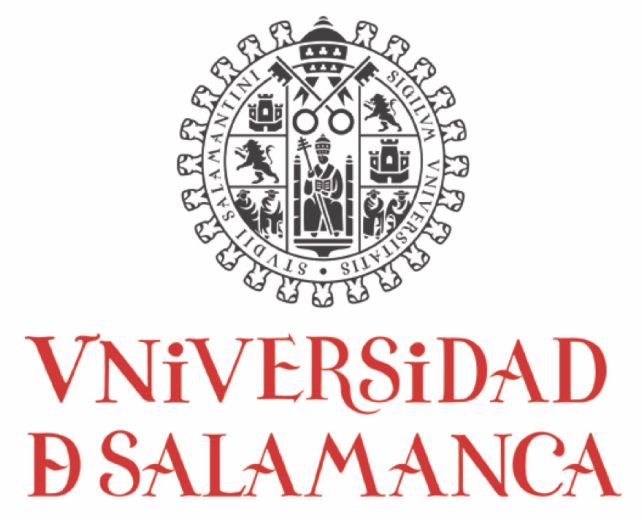

CAMPUS DE EXCELENCIA INTERNACIONAL

Marine Huault

Department of Applied Physic

University of Salamanca

A thesis submitted for the degree of

Doctor of Philosophy at the Universidad de Salamanca

Supervisors: Prof. Luca Volpe \& Prof. Luis Roso 



\section{Declaration}

I, Marine HUAULT, declare that this thesis titled "Laser-driven proton acceleration and detection at high repetition rate" and the work presented in it are my own. I confirm that:

- This work was done wholly or mainly while in candidature for a research degree at this University.

- Where any part of this thesis has previously been submitted for a degree or any other qualification at this University or any other institution, this has been clearly stated.

- Where I have consulted the published work of others, this is always clearly attributed.

- Where I have quoted from the work of others, the source is always given. With the exception of such quotations, this thesis is entirely my own work.

- I have acknowledged all main sources of help.

- Where the thesis is based on work done by myself jointly with others, I have made clear exactly what was done by others and what I have contributed myself.

Signed:

Date: Salamanca, 28-04-2021 
I would like to dedicate this thesis to my parents and my brother. 


\section{Acknowledgements}

I would like to acknowledge the people who had a decisive role in the realization of this thesis work, by sharing their scientific skills and giving me their personal support.

First of all, I would like to thank my PhD directors, Luis Roso and Luca Volpe for all their support and encouragement they provided throughout my $\mathrm{PhD}$, for teaching me physics and giving me the opportunity to be involved in different research activities during my thesis.

Secondly, my thanks go to Giancarlo Gatti, head of the scientific division, for helping me in the realization of the thesis and from whom I learned so much.

I would like also to thank Dimitri Batani, without whom my $\mathrm{PhD}$ in Salamanca wouldn't have started. Special thanks for his support and encouragement during all these years.

Special thanks to all my colleagues of CLPU, from the scientific area and from other departments, in particular Carlos Salgado, Jon Imanol Apiñaniz, Diego De Luis, Ainoha Mantaut, Ana Cives Fernández, Jose Antonio Pérez-Hernández, Pilar Puyuelo Valdes, José Luis Henares and Michael Touati for their precious help during my $\mathrm{PhD}$, for their personal support and for sharing great time at the CLPU.

This work wouldn't have been possible without the collaboration with other research institutions. I would like to thank Carolina Gutiérrez Neira and Nuria Gordillo for their kindness and competence during the scintillator characterization at the CMAM. I also would like to thank Josefine Metzkes-Ng and the HZDR team for their precious help and great work in the realization of the experiment. Special thanks to Diego de Luis for his major role in the realization of these experiments with his incredible skills in building the scintillator detector.

I would like to thank Robert Fedosejevs for always been available to answer my questions, with kindness and patience, and from whom I learned a lot. 
My thanks also go to the engineer, radiation protection and laser team from CLPU, without whom the experiments would not have been possible.

Thanks go to the CLPU for the financial support to the experiments abroad and giving me the opportunity to assist to important conferences and schools during these years at CLPU. My activities within the laboratory have been a great novelty every day and have always represented an opportunity for development, both personal and professional. 


\section{Abstract}

\section{Laser-driven proton acceleration and detection at high repetition rate}

For more than two decades, laser-driven proton acceleration has been an important field of research with a potential interest for several applications in different fields of physics, chemistry and material science as well as biomedical and cultural heritage. However, the laser-matter interaction is a complex process which is still not totally controlled and the resulting interaction depends mainly on laser and plasma parameters. Still many studies are carried out to understand the process behind this mechanism through the characterization of the spectral and spatial properties of the proton beam. With the advent of high power lasers working at high repetition rate, a careful development of particle diagnostics suitable for online shot-to shot analysis is therefore essential.

The $\mathrm{PhD}$ thesis focuses on the generation, transport and detection of laser-driven proton source. The theory of protons and fast electrons driven by the interaction of ultra-intense laser pulse in overdense plasma will be explored in the first part of the thesis. In a second part, the development of a scintillator-based proton detector, able to measure both the proton beam energy and its spatial distribution and capable of being set in a high repetition mode is presented. The detector has been designed and built at the Spanish Center for Pulsed Lasers (CLPU) and tested in collaboration with facilities across the EU. The work related to the development of this new diagnostic, including both theoretical and experimental investigations, is described in the thesis. The final part of the thesis is dedicated to the commissioning experiments of the petawatt laser system VEGA 3, which has recently started the operation phase. Implementation of our scintillator detector and preliminary results of the experiment are presented.

Keywords: Scintillator-based proton detector, Laser-driven proton sources, High Repetition Rate laser system 


\section{Extracto (Spanish Abstract)}

Durante más de dos décadas, la aceleración de protones impulsada por láser ha sido un campo importante de investigación con un interés potencial para varias aplicaciones en diferentes campos de la física, la química y la ciencia de los materiales, así como en el área biomédica y el patrimonio cultural. Sin embargo, la interacción láser-materia es un proceso complejo que todavía no está totalmente controlado y la interacción resultante depende principalmente de los parámetros del láser y del plasma. Se están desarrollando diverses actuaciones para comprender el proceso detrás de este mecanismo a través de la caracterización de las propiedades espectrales y espaciales del haz de protones. Con la llegada de los láseres de alta potencia que funcionan con alta repetición, por lo tanto, es esencial un desarrollo cuidadoso de diagnósticos de partículas adecuados para el análisis en tiempo real de disparo a disparo.

La tesis doctoral se centra en la generación, transporte y detección de una fuente de protones generada por láser. En la primera parte de la tesis se explorará la teoría de los protones y los electrones rápidos generados por la interacción de un pulso láser ultra intenso en un plasma sobredenso. En la segunda parte, se presenta el desarrollo de un detector de protones basado en centelleo, capaz de medir tanto la energía del haz de protones como su distribución espacial y capaz de operar en un modo de alta repetición. El detector ha sido diseado y construido en el Centro de Láseres Pulsados (CLPU) y probado en colaboración con instalaciones europeas. El trabajo relacionado con el desarrollo de este nuevo diagnóstico, incluidas las investigaciones tanto teóricas como experimentales, se describe en la tesis. La parte final de la tesis está dedicada a los experimentos de puesta en marcha del sistema láser de petavatio VEGA-3. Se presentan la implementación de nuestro detector de centelleo y los resultados preliminares del experimento.

Keywords: Detector de protones basado en centelleo, fuentes de protones generadas por láser, sistema láser de alta tasa de repetición. 


\section{Contents}

Publications and research activities $\quad x i$

1 Introduction 1

1.1 Context .......................... 1

1.2 Goal of the thesis . . . . . . . . . . . . . . . . 4

2 Laser-Matter Interaction: State of the art $\quad 7$

2.1 Short Laser Pulses . . . . . . . . . . . . . . . . . 7

2.1.1 Laser Basics . . . . . . . . . . . . . . . . . . . . . 7

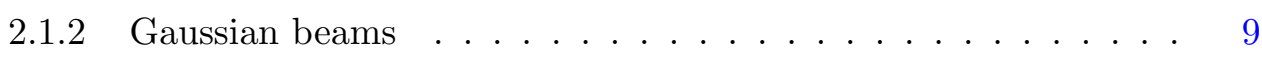

2.1.3 Femtosecond Laser Pulse generation . . . . . . . . . . . . . . 11

2.2 Laser-Plasma processes . . . . . . . . . . . . . . . . 13

2.2 .1 Introduction to plasma . . . . . . . . . . . . . . 13

2.2 .2 Ionization processes . . . . . . . . . . . . . . . . 14

2.2.3 Plasmas characteristic lengths . . . . . . . . . . . . . 15

2.2.4 Collective oscillations: the plasma Frequency . . . . . . . . . 18

2.2.5 Plasma parameters . . . . . . . . . . . . . . . . . 19

2.2 .6 Electromagnetic interaction with plasma . . . . . . . . . 20

3 Laser-Driven proton sources $\quad 23$

3.1 State of the art . . . . . . . . . . . . . . . 2 23

3.2 Laser-driven fast electrons in solid target . . . . . . . . . . . . . 25

3.2.1 Single Electron Motion in an Ultra-High Intense Laser Pulse . 26

3.2 .2 Ponderomotive Force . . . . . . . . . . . . . . . . . . 30

3.2 .3 Heating Mechanisms . . . . . . . . . . . . . . . . . 32

3.2.4 Fast electron beam transport . . . . . . . . . . . . . 37

3.2.4.1 Collisional effects . . . . . . . . . . . . . 37

3.2.4.2 Collective Effects . . . . . . . . . . . . . . . . . 43

3.3 Target Normal Sheath Acceleration . . . . . . . . . . . . . . . . 45

3.3 .1 Mechanism . . . . . . . . . . . . . . . 46

3.3.2 Main experimental achievements . . . . . . . . . . . . 49

3.3.3 Beam optimization . . . . . . . . . . . . . . . . 51

3.4 Proton interaction with matter . . . . . . . . . . . . . 54 
3.4.1 Proton Stopping Power theory _. . . . . . . . . . . . . 55

3.4 .2 Coulomb Multiple scattering . . . . . . . . . . . . 57

3.5 Proton beam Diagnostics . . . . . . . . . . . . . . . 58

3.5.1 Time of Flight techniques . . . . . . . . . . . . . 58

3.5.2 Magnetic spectrometer (Thomson Parabola spectrometer) . . . 62

3.5.3 Passive Ion sensitive detectors . . . . . . . . . . . . . . 65

3.5.4 Toward active diagnostics: Fast scintillators . . . . . . . . . . 67

4 A 2D scintillator-based proton detector for high repetition rate ex$\begin{array}{ll}\text { periments } & 73\end{array}$

4.1 Context and recent developments . . . . . . . . . . . . . 73

4.2 A Full 2D-scintillator-based proton detector . . . . . . . . . . 75

4.2 .1 case $L_{0}=0 \ldots \ldots \ldots \ldots \ldots \ldots \ldots \ldots \ldots$

$4.2 .2 \quad$ case $L_{0} \neq 0 \ldots \ldots \ldots \ldots \ldots \ldots \ldots \ldots \ldots$

4.2.3 Spatial-energy dependence of the proton beam divergence . . . 79

4.2.4 Monte Carlo code for numerical simulation (FLUKA) . . . . . 79

4.2 .5 Discussion . . . . . . . . . . . . . . . . . 80

4.3 Characterization of the scintillator detector at the tandem accelerator

of the CMAM . . . . . . . . . . . . . . . . . 80

$4.3 .1 \quad$ CMAM facility . . . . . . . . . . . . . . . 81

4.3.2 Characterization of the BC-400 plastic scintillator response . . 81

4.3.3 Implementation and preliminary study of the detector with a

collimated and mono-energetic proton beam . . . . . . . 90

4.4 Implementation of the detector in laser-driven proton acceleration ex-

periment at the HZDR facility . . . . . . . . . . . . . 97

4.4 .1 DRACO LASER SYSTEM . . . . . . . . . . . . 97

4.4.2 Experimental set-up . . . . . . . . . . . . . . . . 97

4.4.3 Proton spectrum reconstruction with the scintillator detector . 102

4.4.4 Proton beam divergence reconstruction . . . . . . . . . 107

4.4.5 Experimental results and discussion . . . . . . . . . . . . . 109

4.5 Conclusion . . . . . . . . . . . . . . . . . . . . 112

5 Application to Laser-driven proton acceleration with petawatt class

laser system at CLPU $\quad 115$

5.1 CLPU VEGA system . . . . . . . . . . . . . . . . . 115

5.1 .1 Pulse contrast . . . . . . . . . . . . . . 116

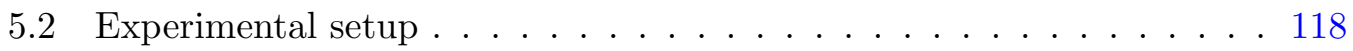

5.2.1 Target and focal spot alignment . . . . . . . . . . . 118

5.2 .2 Overview of the diagnostic system . . . . . . . . . . . . . . 120

5.3 Experimental results . . . . . . . . . . . . . . . . . . . . . . . . 122

5.4 Conclusion . . . . . . . . . . . . . . . . . . 126

6 Conclusions and Perspectives 129 
7 Resumen y conclusiones de la tesis

131

$\begin{array}{ll}\text { Appendix A } & \mathbf{1 3 5}\end{array}$

.1 PN and PIN junction Photodiodes ............... . . 135

$\begin{array}{ll}\text { Appendix B } & 139\end{array}$

$.2 \quad$ Draco laser system . . . . . . . . . . . . . . . . . . . . . . . . . . 139

$\begin{array}{lr}\text { Bibliography } & 141\end{array}$

e 


\title{
Publications and research activities
}

\author{
Articles: (4)
}

- F. Mirani, A. Maffini, F. Casamichiela, A. Pazzaglia, A. Formenti, D. Dellasega, V. Russo, D. Vavassori, D. Bortot, M. Huault, G. Zeraouli, V. Ospina, S. Malko, J. I. Apiñaniz, J.A. Pérez-Hernández, D. De Luis, G. Gatti, L. Volpe, A. Pola and M. Passoni. "Integrated quantitative PIXE analysis and EDX spectroscopy using a laser-driven particle source." Science advances, EABC8660, January 2021. DOI: http://doi.org/10.1126/sciadv.abc8660

Personal contribution: Experimental setup installation, laser alignment, data acquisition during the experiment.

- J. Pasley, G. Andrianaki,J. I. Apiñaniz, A. Baroutsos, D. Batani, E. P. Benis, A. Ciardi, D. Cook, V. Dimitriou, B. Dromey, I. Fitilis, G. Gatti, A. Grigoriadis, M. Huault, J. A. Pérez-Hernández, E. Kaselouris, O. Klimo, M. Koenig, G. Koundourakis, M. Kucharik, J. Limpouch, R. Liska, C. Salgado Lopez, S. Malko, S. Olmos-Migueláñez, Y. Orphanos, V. Ospina, N. A. Papadogiannis, S. Petrakis,J. Psikal, M. Rico, M. Serena Rivetta, M. J Rodríguez-Conde, J. J Santos, M. Sinor, A. Skoulakis, I. Tazes, L. T Pascual, M. Touati, C. Tsitou, P. Vachal, L. Volpe, J. Vyskocil, S. White, M. Yeung, G. Zeraouli, and M. Tatarakis. "Innovative education and training in high power laser plasmas (PowerLaPs) for plasma physics, high power laser matter interactions and high energy density physics: experimental diagnostics and simulations." High Power Laser Science and Engineering, Vol. 8, e9, February 2020. DOI: https://doi.org/10.1017/hpl.2020.12

Personal contribution: Oral presentation at the PowerLaPs training school, March 25th-29th, 2019, held at CLPU, Salamanca, Spain. Title of the talk: "Laser driven ion acceleration at VEGA-II." 
- L. Volpe, R. Fedosejevs, G. Gatti, J. A. Pérez-Hernández, C. Méndez, J. I. Apiñaniz, X. Vaisseau, C. Salgado, M. Huault, S. Malko, G. Zeraouli, V. Ospina, A. Longman, D. De Luis, K. Li, O. Varela, E. García, I. Hernández, J.D. Pisonero, J. García Ajates, J. M. Alvarez, C. García, M. Rico, D. Arana, J. Hernández-Toro and L. Roso. "Generation of high energy laser-driven electron and proton sources with the $200 \mathrm{TW}$ system VEGA 2 at the Centro de Laseres Pulsados." High Power Laser Science and Engineering, Vol 7, e25, January 2019. DOI: https://doi.org/10.1017/hpl.2019.10

Personal contribution: Experimental setup installation, laser alignment, data acquisition during the experiment and analysis.

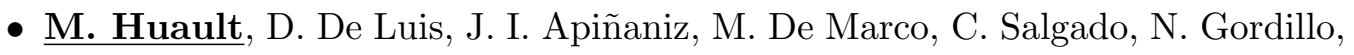
C. Gutiérrez Neira, J.A. Pérez-Hernández, R. Fedosejevs, G. Gatti, L. Roso and L. Volpe. "A 2D scintillator-based proton detector for high repetition rate experiments." High Power Laser Science and Engineering, Volume 7, e60, September 2019. DOI: https://doi.org/10.1017/hpl.2019.43

Personal contribution: Experimental setup installation, carrying out the experiment as Co-PI, data acquisition, analysis and writing of the manuscript.

\section{Oral contributions: (5)}

- M. Huault, L. Volpe, R. Fedosejevs, G. Gatti, J. A. Pérez-Hernández, C. Méndez, J. I. Apiñaniz, X. Vaisseau, C. Salgado, S. Malko, G. Zeraouli, V. Ospina, A. Longman, D. De Luis, K. Li, O. Varela, E. García, I. Hernández, J.D. Pisonero, J. García Ajates, J. M. Alvarez, C. García, M. Rico, D. Arana, J. Hernández-Toro and L. Roso. "Ion diagnostics for High Repetition Rate experiments." $3^{\text {rd }}$ Laser Plasma Summer School (LaPlaSS) - September $14^{\text {th }} 18$ th, 2020 - Salamanca, Spain.

Personal contribution: Experimental setup installation, laser alignment, carrying out the experiment, data acquisition, analysis.

- M. Huault, J. I. Apiñaniz, D. De Luis, M. De Marco, R. Fedosejevs, J.A. Pérez-Hernández, M. Touati, J. Metzkes, U. Schramm, K. Zeil, N. Gordillo, C. Gutiérrez Neira, G. Gatti, L. Roso and L. Volpe. "A $2 D$ scintillator based proton detector for High Repetition Rate experiments." Beam Line and Instrumentation Workshop (BLIN4) - June 2020 - Salamanca, Spain.

Personal contribution: Experimental setup installation, carrying out the experiment as Co-PI, data acquisition during the experiment, analysis and oral presentation.

- L. Volpe, M. Huault, J. I. Apiñaniz, D. De Luis, M. De Marco, R. Fedosejevs, J. A. Pérez-Hernández, J. Metzkes, U. Schramm, K. Zeil, N. Gordillo, C. Gutiérrez Neira, G. Gatti, L. Roso. "Two-dimensional high Repetition Rate Scintillator-based Proton detector." JRA Meeting - October 2019 - Florence, 
Italy.

Personal contribution: Experimental setup installation, carrying out the experiment as Co-PI, data acquisition during the experiment and analysis.

- S. Malko, W. Cayzac, V. Ospina, X. Vaisseau, J. I. Apiñaniz, D. Batani, M. Barriga-Carrasco, R. Fedosejevs, M. Huault, P. Neumayer, G. Prestopino, C. Verona, J.A. Pérez-Hernández, R. Ramis and L. Volpe. "Stopping power measurements of ions in a moderately coupled and degenerate plasma." $61^{\text {st }}$ Annual Meeting of the APS Division of Plasma Physics - October, 2019 - Fort Lauderdale, Florida, USA.

Personal contribution: Experimental setup installation, laser alignment, data acquisition during the experiment.

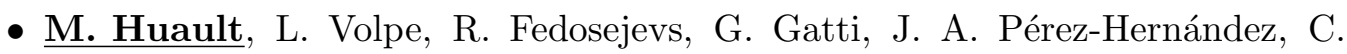
Méndez, J. I. Apiñaniz, X. Vaisseau, C. Salgado, S. Malko, G. Zeraouli, V. Ospina, A. Longman, D. De Luis, K. Li, O. Varela, E. García, I. Hernández, J.D. Pisonero, J. García Ajates, J. M. Alvarez, C. García, M. Rico, D. Arana, J. Hernández-Toro and L. Roso. "Ion diagnostics for High Repetition Rate experiments." $2^{\text {nd }}$ Laser Plasma Summer School (LaPlaSS) - September $16^{\text {th }}-20$ th, 2019 - Salamanca, Spain.

Personal contribution: Experimental setup installation, laser alignment, carrying out the experiment, data acquisition, analysis.

- C. Salgado, M. Ehret, V. Ospina, J.A. Pérez-Hernández, M. Huault, M. De Marco, J. I. Apiñaniz, F. Hannachi, D. De Luis, J. HernándezToro, C. Méndez, J.D. Pisonero, O. Varela, A. Debayle, E. dHumières, L. Gremillet, T.-H. NguyenBui, E. Olivier, G. Revet, C. Brabetz, J. Caron, T. Ceccotti, R. Nuter, D. Raffestin, M. Roth, G. Schaumann, X. Vaisseau, G. Gatti, L. Volpe, and J.J. Santos. "Near-critical density plasmas from supersonic gas jets for enhanced ion acceleration by ultra-intense laser interaction." 11th International Conference on Inertial Fusion Science and Applications (IFSA2019) - September 2019 - Osaka, Japan.

Personal contribution: Experimental setup installation, laser alignment, data acquisition during the experiment.

- S. Malko, W. Cayzac, V. Ospina, X. Vaisseau, J. I. Apiñanizaniz, D. Batani, M. Barriga-Carrasco, R. Fedosejevs, M. Huault, P. Neumayer, J.A. PérezHernández, G. Prestopino, R. Ramis, C. Verona and L. Volpe. "Ion stopping power measurements in a moderate and degenerate plasma." International Conference on High Energy Density (ICHED2019) - March 2019 - University College Oxford, UK.

Personal contribution: Experimental setup installation, laser alignment, data acquisition during the experiment.

- M. Huault, L. Volpe, R. Fedosejevs, G. Gatti, J. A. Pérez-Hernández, C. Méndez, J. I. Apiñaniz, X. Vaisseau, C. Salgado, S. Malko, G. Zeraouli, V. 
Ospina, A. Longman, D. De Luis, K. Li, O. Varela, E. García, I. Hernández, J.D. Pisonero, J. García Ajates, J. M. Alvarez, C. García, M. Rico, D. Arana, J. Hernández-Toro and L. Roso. "Laser Driven Ion Acceleration at VEGA-II." Innovative Education \& Training in High Power Laser Plasmas (PowerLaps) March $25^{\text {th }}-29^{\text {th }}, 2019$ - Salamanca, Spain.

Personal contribution: Experimental setup installation, laser alignment, carrying out the experiment, data acquisition, analysis. 


\section{Chapter 1}

\section{Introduction}

\subsection{Context}

For more than 50 years, the interest in laser technology has not stopped evolving, motivated in particular by the field of experimental research requiring always greater laser intensities, today exceeding $I_{L} \lambda^{2}>10^{21} \mathrm{~W} \cdot \mu \mathrm{m}^{2} . \mathrm{cm}^{-2}$. These high intensities have been achieved thanks to the discovery of powerful Q-switching and mode-locking techniques, enabling ultrashort pulse generation low to femtosecond. A breakthrough in laser technology was achieved by Donna Strickland and Gerard Mourou in 1985 (Nobel Prize 2018) [Strickland \& Mourou, 1985], by developing the chirped pulse amplification (CPA) technique, then making possible to obtain ultra-short laser pulses down to the femtosecond range with an ultra-high power from terawatt to petawatt.

Beyond an intensity of $10^{18} \mathrm{~W} . \mathrm{cm}^{-2}$, the laser-matter interaction regime becomes relativistic and electrons are able to follow the oscillations of the laser field by moving at a quiver velocity close to the speed of light. The interaction of a high intensity laser with a thin target (mainly solid) creates a plasma, a partially or completely ionized state of matter, with interesting properties allowing it to withstand extreme currents and fields that conventional accelerators can't sustain due to electrical breakdown. At such high intensities, high-energy proton acceleration is possible. Interest in laser driven proton acceleration began with three main independent experiments done in 2000 [Clark et al., 2000; Maksimchuk et al., 2000; Snavely et al., 2000] where proton energy close to $60 \mathrm{MeV}$ have been registered [Snavely et al., 2000]. The latter highlighted that this acceleration was the result of complex mechanisms relying on the energy transfer from the electrons, acting as intermediaries in the process. The mechanism responsible for this effect will be later introduced as Target Normal Sheath Acceleration (TNSA) [Wilks et al., 2001], where protons are accelerated up to energies of several tens of $\mathrm{MeV}$ in strong electric fields exceeding $\mathrm{TV} / \mathrm{m}$ over short ( $\mu \mathrm{m}$-scale) distances. This is around 3 orders of magnitude higher than the ones produced by conventional accelerators, with an electric field limited to about $100 \mathrm{MV} / \mathrm{m}$ due to breakdown voltages. Further studies, such as target geometry development led 
to reach proton energy records of $67.5 \mathrm{MeV}$ [Gaillard et al., 2011], $85 \mathrm{MeV}$ [Wagner et al., 2016] and lastly $>94 \mathrm{MeV}$ [Higginson et al., 2018]. Favorable properties of laser driven ion source include small source size, high brightness, small divergence, short bunch duration and broad band energetic spectrum thus making laser-based techniques ideal candidates for the development of compact, economical particle acceleration techniques, complementary to the particle beam sources typically produced in conventional accelerators.

These unique properties open the way to several applications for laser-driven ion beams. One of the most targeted stays the oncological hadrontherapy [Bulanov \& Khoroshkov, n.d.]. Indeed, proton and ions have the property to deliver most of their energy close to the end of their path (in the so-called Bragg peak). Therefore, at the difference with electron or X-rays, the dose can be delivered precisely to the tumour, avoiding unnecessary irradiations of surrounding healthy cells. For this property, laser-driven ion beams have also been proposed for the use in fast ignition inertial confinement fusion. In particular, the team of [Roth et al., 2001] proposed to use laser-accelerated proton beam to ignite the hot spot in the fuel pellet.

Again for medical interest, the interaction of $\mathrm{MeV}$ protons with special targets can produce short-lived isotopes for positron emission tomography (PET) diagnostics [Fritzler et al., 2003; Santala et al., 2001]. PET produces three-dimensional image of physiological processes happening in the body. It consists of the detection of gamma rays emitted indirectly by a positron-emitting radionuclide, known as tracer. Laserdriven ion accelerators represent a more simplified approach to isotope production with a capability similar to that of a cyclotron and allowing on-site installations at the therapy centers.

Interest in laser driven proton sources for material science applications keep growing. For example, the laser-based particle induced X-ray emission (Laser-PIXE) analysis technique is a non-destructive material characterization method and is very useful for multi-elemental analysis. Nowadays, it is widely used in several fields including (but not limited to) biology, cultural heritage, agriculture, geology, petrology, environmental study, etc...Several groups are working on this technique and recent studies have been published by the team of [Barberio \& Antici, 2019] and recently by[Mirani et al., 2021] (performed at the Spanish Center for Pulsed Lasers CLPU with the VEGA 2 laser), presenting the first quantitative laser-driven PIXE analysis.

However, one of the main difficulty existing on laser installations is the non-linearity of the phenomena involved in the laser-matter interaction, thus limiting the shotto-shot stability of the produced beams. In order to be able to use such sources of proton produced by laser-plasma interaction for application, it is essential to properly characterize both the angular and energy distributions of the protons, shot by shot. The development of the CPA technique extended the possibility to have femtosecond pulses reaching the same laser intensities obtained with previous laser systems (ranging from nanoseconds to picoseconds time domain), with the possibility to work 
at HRR. This last point is crucial, since it allows to obtain a sufficient high particle flux driven by laser to be comparable to that generated by conventional accelerators. With the advent of HRR laser facilities, the main challenge is the diagnostic adaptation for real-time particle characterization. Nowadays, several laboratories among which CLPU (VEGA), HZDR (DRACO), ELI (L3 HAPLS), RAL (Gemini), INRS (ALLS), Berkeley LAB (BELLA) are in possession of ultra-intense and ultra-short laser pulse with the capacity of delivering several joules in the 1-10 $\mathrm{Hz}$ system regime.

CLPU is a facility specialized in ultra-short and ultra-intense laser pulses. The main activity of the center is to provide high power laser to the scientific community with an open access to national and international users, as well as to promote scientific, technical and innovation development by collaborating with international laboratories. The main laser system of CLPU is VEGA, a Ti:Sa laser system recently operative and providing a full Petawatt VEGA-3 $(30 \mathrm{~J}, 30 \mathrm{fs}, 1 \mathrm{~Hz}), 200$ TW VEGA-2 (6 J, 30 fs, $10 \mathrm{~Hz})$ and $20 \mathrm{TW}$ VEGA-1 $(600 \mathrm{~mJ}, 30 \mathrm{fs}, 10 \mathrm{~Hz})$ laser outputs.

The VEGA 2 laser started to be operative for external users in early 2018. As a convenience, it is able to provide to the users secondary sources of particles and radiations, as well as the necessary diagnostics for their studies and characterizations. VEGA 2 can be focused onto solid target of few microns (3-10 $\mu \mathrm{m}$ thick target) and protons up to $8 \mathrm{MeV}$ have been generating via TNSA mechanism [Volpe et al., 2019] . The commissioning experiment on VEGA-3 has been recently done with solid target and preliminary results will be presented in this thesis.

The CLPU directs its own research and expertise for online particle detection and characterization, in order to fully use the VEGA HRR performance. For example, a Kirkpatrick-Baez microscope for X-ray detection [Zeraouli et al., 2019], a multi-pin hole Thomson Parabola spectrometer and magnetic proton energy selector [Apiñaniz et al., 2021] have been developed by the CLPU team.

Although Thomson parabola spectrometer or TOF diagnostics available at CLPU can provide an online high resolution proton spectra, only a small part of the proton beam can be collected due to their small solid angle of detection. In addition, in order to evaluate our laser system performance and characterized the mechanisms responsible for the proton acceleration, absolute calibrated diagnostics are required. The radiochromic film (RCF) is an ideal tool to measure fine structures in proton beam profiles since it gives information of angular and spectral distribution of the entire beam. When calibrated, it is possible to obtain the total particle number of the accelerated proton pulse. However, RCF is a passive detector and becomes inappropriate when a direct feedback of the results at HRR is needed since it cannot be readout online.

CLPU is still working on targetry and diagnostic developments to match the VEGA laser system repetition rate potential. Indeed, target movement and alignment still need to be enhanced for working modes above $0.1 \mathrm{~Hz}$ while current diagnostics for the HRR acquisition still cannot reproduce the performance of conventional passive detectors such as RCF. The possibility of extending this technique to the HRR mode 
of operation is nowadays a challenge in the laser-plasma community, and several laboratories and research groups are working on this. The first $2 \mathrm{D}$ scintillator-based proton detector for high repetition rate experiments has been developed at CLPU and will be presented in this thesis. The detector design has been done by considering an extension at HRR of the RCF stack passive detector by using scintillator layers capable of converting the proton energy deposition into light emission to be then collected by a CCD camera. Such detector is able to give direct feedback on both the proton energy and its transversal spatial distribution along the propagation axis [Huault et al., 2019]. It paves the way for a new class of on-line detectors in laser-plasma physics experiments. The detector design, construction and operation is a relevant part of the presented work.

\subsection{Goal of the thesis}

The main goal of this work is to establish a proton source generated by laser and working at High Repetition Rate (HRR) for several applications. The proton source is produced at the CLPU where a multi Petawatt-class laser VEGA is installed. Proton diagnostics methods are investigated with a strong emphasis on the development of HRR compatible diagnostics such as scintillator-based proton detector able to characterize simultaneously energy spectrum and spatial distribution.

The scintillator-based detector has been designed and built at the CLPU and tested in collaboration with facilities across the EU. The work related to the development of this new diagnostic, including both theoretical and experimental investigations, is described in the first part of the thesis as well its operation in different laser facilities. In addition, the development and optimization of a stable proton source driven by the VEGA 3 laser system is presented and explained in the thesis.

The structure of this thesis is as follows:

\section{- Chapter 2}

In this chapter, the physical principles of generation of short and intense laser pulses is firstly exposed. We will then look over the theoretical aspects of lasermatter interaction with a brief description of the plasma generated by laser, its characteristics and fundamental parameters.

\section{- Chapter 3}

Chapter 3 presents an overview of laser-driven proton source. It includes some theoretical background about electron heating mechanisms and their transport in solid target, responsible of the charge separations and the acceleration of the ionic species. In the continuity, a brief overview of laser-driven proton beams generation by TNSA and their transport in matter will be introduced to finally get into the main proton diagnostics used during the thesis study. 


\section{- Chapter 4}

This chapter is dedicated to the theoretical and experimental study of a scintillatorbased detector able to measure the proton energy and the spatial distribution at HRR. The first test of the detector done with a conventional proton source at the tandem accelerator from the Centro de Micro-Análisis de Materiales (CMAM) located in Madrid and its application to laser-driven proton experiment, carried out at the HZDR facility located in Dresden will be presented.

\section{- Chapter 5}

Finally, the last part of the thesis is dedicated to the commissioning experiments of VEGA-3 Petawatt laser system, which has recently started the operational phase. Implementation of our scintillator detector in the experiment and preliminary results are presented. 


\section{Chapter 2}

\section{Laser-Matter Interaction: State of the art}

The interaction of high intensity light with matter has been studied since the first invention of the laser technology in the early 60's [Maiman, 1960]. Since then, lasers are in constant development, particularly in industrial applications, communication, medicine, fundamental physics, etc...with the discovery and development of CPA (Chirped Pulse Amplification) technology in 1985 by [Strickland \& Mourou, 1985] (2018 Nobel prize), progress in ultra-short pulse laser technology has only increased, opening the field of relativistic laser-plasma physics with intensities $>10^{18} \mathrm{~W} . \mathrm{cm}^{-2}$ with pulses in the ps, sub ps and fs range. In this chapter, the physical principles of generation of short and intense laser pulses is firstly exposed. We will then look over the important laser parameters in the context of laser-plasma interaction and we will end up the chapter with a brief description of the plasma generated by laser, its characteristics and fundamental parameters.

\subsection{Short Laser Pulses}

\subsubsection{Laser Basics}

The acronym "LASER" stands for Light Amplification by Stimulated Emission of Radiation. The laser emission is based on the interaction between an electromagnetic wave and a material medium (amplifying medium), where, in the presence of the electromagnetic field, an excited atom from the medium emits a photon identical to the incident photon (same energy, phase and direction as the incident photon). This process is called "Stimulated Emission" and was discovered by [Einstein, 1917] in 1917. In 1950, [Kastler, 1950] proposed a technique for the optical pumping and population inversion and 3 years later, the first Maser (Microwave Amplification by Stimulated Emission of Radiation) of Ammoniac gas was built by Gordon, Zeiger and Townes [Gordon et al., 1955] followed in the next months by [Basov \& Prokhorov, 1956]. The first laser was built in 1960 by [Maiman, 1960], based on theoretical work 
by [Schawlow \& Townes, 1958]. To obtain the stimulated emission, three main parts are needed: the amplifying medium, a pumping system and a cavity.

\section{Amplifying medium}

An amplifying medium can be made of very diverse composition (atom, molecule, ion, liquid, solid, semiconductor, gases, etc...) and this is where the amplification of radiation occurs. Let's consider two electronics levels 1 and 2 with the energy $E_{1}$ and $E_{2}$ respectively the fundamental and excited state (see figure 2.1). Three mechanisms are essential in the process of amplification: the first one, called absorption occurs when a photon of energy $h \nu=E_{1}-E_{2}$ transfers its energy to an electron of an atom in state $E_{1}$. The electron is now in an excited state $E_{2}$, with a probability to loose spontaneously its energy and fall back to the lower state $E_{1}$, while emitting a photon of energy $h \nu$. This process is known as spontaneous emission. Einstein came out in 1917 with the hypothesis that two types of transition were not enough to describes the radiation equilibrium, considering the Planck Law. He showed that a third mechanism of radiation transition should occurs, called the stimulated emission. When a photon of energy $h \nu$ interacts with an excited atom having an electron in the state $E_{2}$, it may stimulate the electron of this atom to fall down to the lower level $E_{1}$ and generate a photon having the same properties than the incident photon. This process is at the origin of the amplification radiation and the laser intensity will be directly proportional to the photon flux.

a)

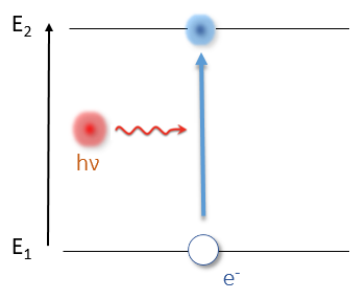

b)

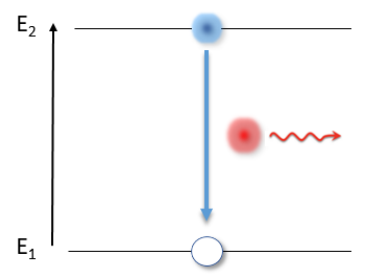

c)

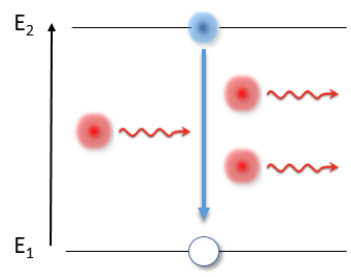

Figure 2.1: The three elementary electron photon interaction processes in atoms: (a) absorption, (b) spontaneous emission, (c) stimulated emission.

\section{Population Inversion and Pumping system}

The generation of photons requires an initial number of atoms in the excited state $E_{2}$ to the detriment of the atoms population of the $E_{1}$ level and it is called "population inversion". The energy needed for the inversion is transferred in the medium externally by a mechanism of "pumping" which can be done by Flash Lamp, laser, chemically, electrically, thermally, etc...where the energy of the photons should match with the energy transition states. Generally, a three-level or four-level system is used to generated the population inversion. Indeed, these systems are composed of two levels inside which the stimulated emission occurs mean while one or two intermediate levels are used to obtain the population inversion. 


\section{Optical Cavity and Laser Oscillation}

The photon amplification occurs when the amplifying medium is placed inside an optical cavity, allowing the photon to get multiplied by going back and forth inside the cavity. The cavity is generally composed by two mirrors in opposition, with one partially reflective (see figure 2.2). A cavity can only confine light in certain positions of the mirrors when it fulfills the stability condition $0<\left(1-L / R_{1}\right)\left(1-L / R_{2}\right)$ where $R_{1,2}$ is respectively the radius of curvature of the mirror 1 and 2 delimiting the cavity length $L$. If the gain is larger than the losses after going back and forth, the intensity of the electromagnetic wave will increase and escape the cavity at a certain intensity threshold. However, the dimension of the cavity imposes some conditions and only

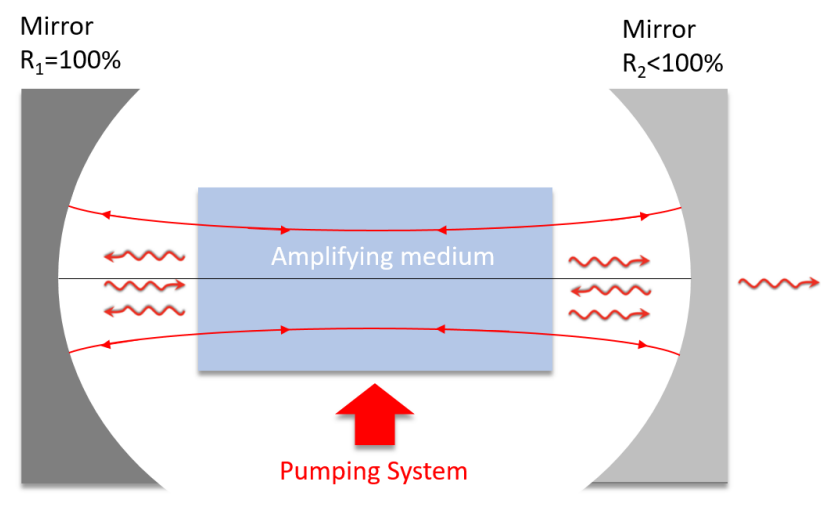

Figure 2.2: Laser cavity scheme.

electromagnetic waves interfering constructively after a round trip will participate to the amplification. Such a wave corresponds to a longitudinal mode (or axial mode) of the cavity and verifies the mode frequency $\nu_{q}=q c / 2 L$ (where $q \in \mathbb{R}$ ).

Variations in the plane transverse to the laser propagation axis inside the cavity determines the spatial coherence of the laser and are called the transverse modes. Contrary to the longitudinal modes that are a time-frequency property, the transverse mode are consequence of spatial coherence of the light propagation. The determination of transverse mode depends on many parameters such as the focusing by mirrors or diffraction cause by aperture during the propagation. Considering a confocal cavity, the mode 00 is the simplest mode and can be described by the light beam propagating back and forth along the axis. The output of a laser oscillating in this mode is a spherical wave with a Gaussian intensity distribution and is called Gaussian mode.

\subsubsection{Gaussian beams}

During the research activities describe on the thesis, pulses with temporal and spatial Gaussian profile are used. The radial intensity distribution in the plane of the 
waist for a Gaussian beam in the fundamental mode (mode 00) is given by

$$
I(r, z)=\frac{2 P}{\pi \mathrm{w}(z)^{2}} \exp \left[\frac{-2 r^{2}}{\mathrm{w}(z)^{2}}\right] \quad\left[\mathrm{W} \cdot \mathrm{cm}^{-2}\right]
$$

where $r$ is the radius position, $\mathrm{w}(z)$ is the Gaussian beam radius at the position $\mathrm{z}$ for which the intensity dropped to $1 / e^{2}$ and $\mathrm{P}$ is the laser beam power.

Considering the paraxial approximation, the complex electric field amplitude of a monochromatic beam propagating along the $\mathrm{z}$ direction is defined by

$$
E(r, z)=E_{0} \frac{\mathrm{w}_{0}}{\mathrm{w}(\mathrm{z})} \exp \left[-\frac{r^{2}}{\mathrm{w}(\mathrm{z})^{2}}\right] \exp \left[i\left(k z+\frac{k r^{2}}{2 R(z)}-\phi(z)\right)\right]
$$

with $E_{0}$ the maximum amplitude, wo the beam waist radius, the wavenumber $k=$ $2 \pi / \lambda_{L}$ and $\phi(z)=\arctan z / z_{R}$ the Gouy phase. The evolution of the laser beam radius $\mathrm{w}(\mathrm{z})$ and its wavefront curvature radius along the propagation direction $\mathrm{z}$ are defined by

$$
\mathrm{w}(\mathrm{z})=\mathrm{w}_{0} \sqrt{1+\left(\frac{z}{z_{R}}\right)^{2}},
$$

and

$$
R(z)=z\left[1+\left(z_{R} / z\right)^{2}\right]
$$

where $\mathrm{w}_{0}$ represents the smaller radius located at the beam waist position and is called waist radius. The term $z_{R}$ is the Rayleigh length and is the distance over which the beam can propagate without diverging significantly. At $z_{R}$ the waist radius $\mathrm{w}_{0}$ increases by a factor $\sqrt{2}$. The latter reads

$$
z_{R}=\frac{\pi \mathrm{w}_{0}^{2}}{\lambda_{L}}
$$

The range in which the laser beam radius is considered to be reasonably collimated is defined by the sum of Rayleigh length at each end of the waist radius

$$
2 z_{R}=2 \frac{\pi \mathrm{w}_{0}^{2}}{\lambda_{L}}=\text { collimated range }
$$

At a large distance $\mathrm{z}$ from the beam waist position, the Gaussian half angle beam divergence reads

$$
\theta=\frac{\lambda_{L}}{\pi \mathrm{w}_{0}}
$$

and is defined as the far-field divergence of the Gaussian beam. It is worth mentioning that in the case of non perfect Gaussian beam, a $\mathrm{M}^{2}$ factor can be introduced as $\mathrm{M}^{2}=\theta \pi \mathrm{w}_{0} / \lambda_{L}\left(\mathrm{M}^{2}=1\right.$ for a Gaussian beam). The propagation of a Gaussian beam is represented in figure 2.3. 


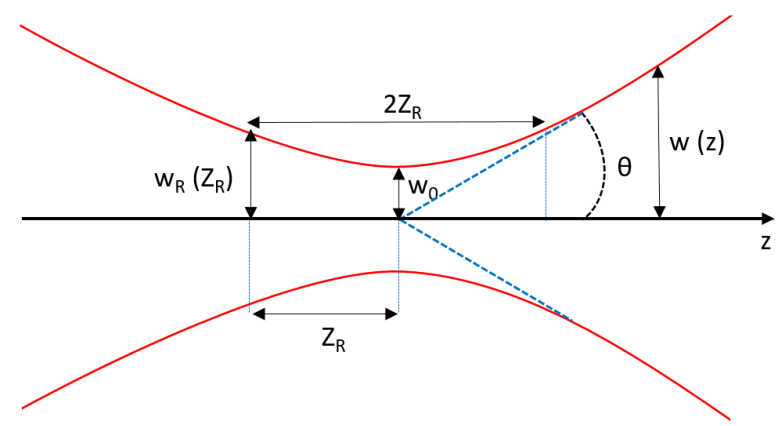

Figure 2.3: Gaussian beam propagation as a function of the distance $\mathrm{z}$ along the beam.

\subsubsection{Femtosecond Laser Pulse generation}

Instead of a continuous or relaxed emission laser which are limited by the recovery time of the system, i.e. the time required to obtain the population inversion, shorter pulses can be obtained by triggering the emission process. The first method historically introduced to obtain shorter-pulse generation was called Q-Switching. It was demonstrated and introduced by [McClung \& Hellwarth, 1962]. It consists in inserting an attenuator inside the cavity that introduce losses in the gain medium. The optical cavity quality factor $\mathrm{Q}$ will initially be kept low to introduces losses while the amplifying medium is being pump. When the population inversion reaches its maximum value, the attenuation is suddenly deleted and the quality factor is restored, all the energy accumulated during the pumping time is then released in a very short light pulse (few nanosecond duration and few Gigawatt peak power). The Q-Switching can be active using Pockels Cells attenuator or passive using saturable absorbers.

Another method called the Mode Locking, allows to reduce consequently the pulse duration to femtosecond. It was introduced in the 70s but was first demonstrated by Hargrove in 1964 [Hargrove et al., 1964]. The mode locking introduces a phase relationship between the longitudinal modes of the cavity, forcing them to constructively interfere, creating a short pulse. We note that the maximum focused intensity that can be generated by the mode locking technique is close to $10^{15} \mathrm{~W} \cdot \mathrm{cm}^{-2}$.

Unfortunately, when the high peak intensity generated with these techniques crosses the gain medium, non-linear effects due to the high fluence of the beam can introduce laser beam wavefront distortions or even cause the destruction of the gain medium, and so limits the amplification. The distortion of the wavefront can be described by the so-called B-integral, representing the phase shift wavefront and should be minimized:

$$
B=\frac{2 \pi}{\lambda_{L}} \int_{0}^{l} n_{2} I_{L}(z) \mathrm{d} z
$$

where $n_{2}$ is the nonlinear index of refraction of the material, $l$ the material length traversed and $I_{L}$ the intensity of the stretched pulse. These optical wavefront distor- 


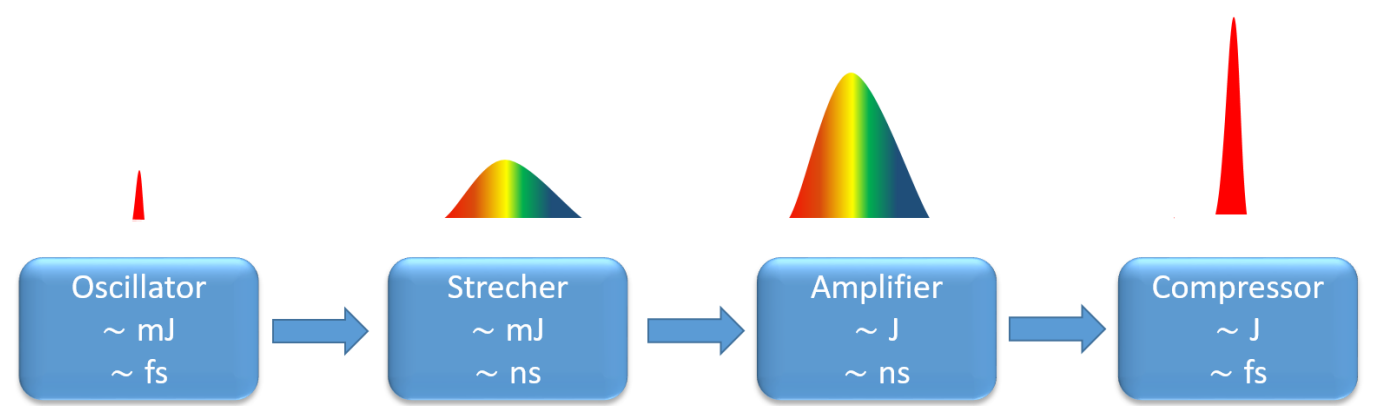

Figure 2.4: CPA techniques.

tions and the ulterior material damage can be effectively prevented by employing the method of Chirp Pulse Amplification (CPA) introduced in 1985 by Strickland and Mourou [Strickland \& Mourou, 1985]. The CPA makes possible to decrease the intensity on the medium and remain below the damage threshold of materials. The first step consists in stretching temporally the low energy laser pulse $(\sim \mathrm{mJ})$ generated through the oscillator (see figure 2.4). The pulse stretcher is composed by dispersive optical elements (diffraction gratings, optical fibers or pair prims, ...) placed so that the low frequency components of the pulse (higher wavelength) travel a shorter path than the high frequency components, allowing to stretch pulses of a factor up to $10^{6}$ times the original pulse duration. The stretched pulse is then sent to a chain of multi-pass power amplifiers, to be amplified up to $10^{9}$ times or more of its initial energy. It is then sent to a compressor, compensating the exact stretching effect in order to resynchronize the different wavelengths and reduce the pulse duration to few tens of femtoseconds. Figure 2.5 shows the laser intensity evolution through time since the early $60 \mathrm{~s}$. The CPA technique is nowadays exploited by nearly all existing high-power, femtosecond systems of the world reaching the Petawatt regime in some facilities (RAL, CLPU, DRACO, ELI, APOLLON, GEKKO, OMEGA, BELLA ...). It is worth mentioning that even if Gaussian beams are considered for simplicity, the transverse profile of multi-watt or petawatt lasers is more similar to a flat top than Gaussian.

Ultra intense laser pulses are most of the time generated together with a non negligible pedestal of nanosecond scale, preceding the main pulse. It is due to the amplification of the spontaneous emission (ASE) in the chain of amplification and it is an unavoidable amplifier noise effect. The ration between the main pulse maximum intensity $I_{0}$ and the ASE pedestal intensity is characterized by the so-called contrast

$$
\text { Contrast }=\frac{I_{A S E}}{I_{0}} \text {. }
$$

Depending on the contrast value, the pedestal intensity can reached $10^{13} \mathrm{~W} . \mathrm{cm}^{-1}$ which is sufficient to ionize a solid density target. Some technologies as Plasma mirror or frequency doubling method can improve the contrast. 


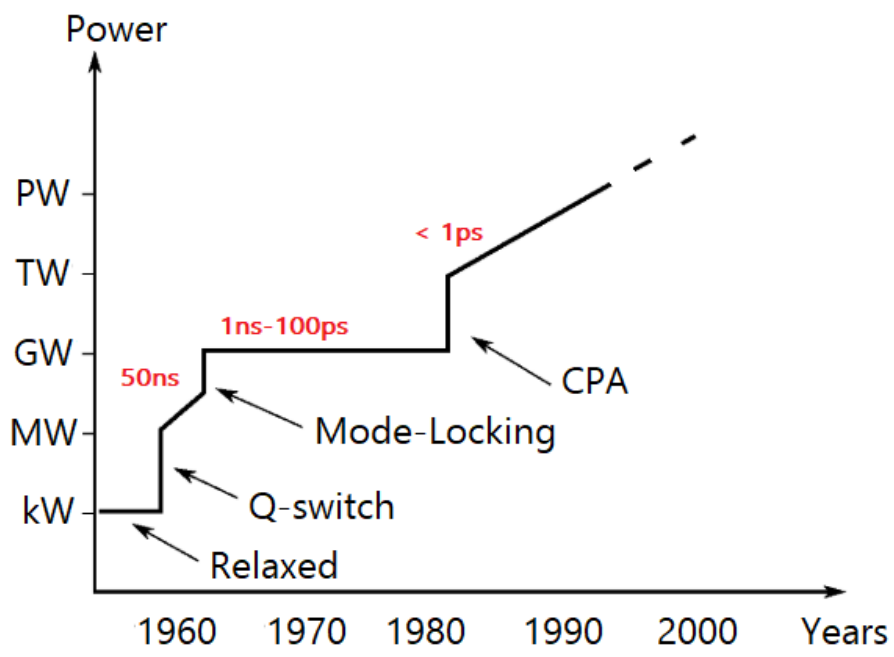

Figure 2.5: Evolution of laser peak power in time. Adapted from [Guemnie-Tafo, 2007].

\section{$2.2 \quad$ Laser-Plasma processes}

\subsubsection{Introduction to plasma}

The plasma, considered as the fourth fundamental state of matter, is composed by atoms at least partially ionized. The term plasma was first introduced by the physicist Irvin Langmuir in the 1920s, who studied electrical discharges in weakly ionized gases. When the matter is heated between $5000 \mathrm{~K}$ and $10000 \mathrm{~K}$, molecules dissociate into atoms by thermal ionization as a result of collision. At temperatures above $10000 \mathrm{~K}$, atoms decompose themselves into electrons and positive ions. Thus plasma constitutes a fourth state of matter. Plasma covers different range of physical system, according to the particle density and temperature, and can be found everywhere in the universe. Partially ionized plasma, where few free electrons and positive ions interact with neutral atoms and molecules, can be founded in the Earth ionosphere, molecular inter-galactic clouds whereas highly ionized and hot plasmas that can be found in stars or in the laboratory where the thermonuclear fusion is controlled. In the frame work of this thesis, we are more interested on laser-driven plasmas. Plasma results to be a very interesting medium of study for its ability to sustain strong electrical $(>\mathrm{GeV} / \mathrm{cm})$ and magnetic fields $(>\mathrm{kT})$. One of its main properties is the collective behavior of the charged particles composing it and is essentially due to the Coulomb interaction force between them. Considering $E_{\text {Coulb }}$ the Coulomb potential energy between two charged particles $q_{1}$ and $q_{2}$ at a distance $r_{12}$ between each other:

$$
E_{\text {Coulb }}(r)=\frac{q_{1} q_{2}}{4 \pi \epsilon_{0} r_{12}} \quad \text { (S.I) }
$$


with $\epsilon_{0} \simeq 8,854 \times 10^{-12} \mathrm{~F} . \mathrm{m}^{-1}$ the vacuum permittivity . Thereby, the movement of each charged particle in a plasma will immediately generate an electromagnetic field which will affect all the other particles in return. This complex mechanism is at the origin of the coherent movement of charged particles, responsible of the generation of oscillations called plasma waves. Due to the reactivity of the plasma to any disturbance, it supports only a small difference in electrical neutrality and is therefore considered quasi-neutral. The mathematical condition reads

$$
-e n_{e}+\sum_{i} Z^{*} e n_{i}=0
$$

with $e \simeq 1,602 \times 10^{-19} \mathrm{C}$ the elementary charge, $n_{e}$ and $n_{i}$ the electron and ion density respectively, and $Z^{*}$ the ionization degree. The possible mechanisms of creating a plasma using a laser are shown in the following section.

\subsubsection{Ionization processes}

The interaction between a high-intensity laser and matter can ionize the latter and form a plasma via different mechanisms according to the laser intensity. For simplicity, they can be described with the Bohr model of the hydrogen atom as a first introduction to Laser-atom interaction [Gibbon, 2005]. The position of the electron is given by the Bohr radius (S.I units)

$$
a_{B}[\mathrm{~m}]=\frac{4 \pi \epsilon_{0} \hbar^{2}}{m_{e} e^{2}}=5.3 \times 10^{-11} .
$$

The electric field reads

$$
E_{a}\left[\mathrm{~V} \cdot \mathrm{m}^{-1}\right]=\frac{e}{4 \pi \epsilon_{0} a_{B}^{2}} \simeq 5.1 \times 10^{11} .
$$

The intensity of an electromagnetic wave with an electric field $E_{a}$ is then given by

$$
I_{a}\left[\mathrm{~W} \cdot \mathrm{cm}^{-2}\right]=\frac{\epsilon_{0} c E_{a}^{2}}{2} \simeq 3.51 \times 10^{16} .
$$

It is known as the atomic intensity and means that for any laser intensity $I_{L}>I_{a}$, the target will be then fully ionized. However, the ionization processes can occur for lower intensity threshold and are defined according to the Keldysh parameter [Keldysh, 1965]

$$
\gamma_{k}=\sqrt{\frac{E_{\text {ion }}}{2 \Phi_{\text {pond }}}}
$$

where $\Phi_{\text {pond }}$ is the so-called ponderomotive potential of the laser field, expressing the effective quiver energy acquired by an oscillating electron where

$$
\Phi_{\text {pond }}=\frac{e^{2} E_{L}^{2}}{4 m_{e} \omega_{L}^{2}}
$$


with $E_{L}$ the laser field and $\omega_{L}$ the laser frequency.

Multiphoton Ionization, $\gamma_{k}>1$

When the interaction of a single photon with an atom is not enough to overcome the potential barrier and eject an electron, the atomic ionization is still possible by a process called Multiphoton Ionization, corresponding to the absorption of several lower frequency photons by the electron. If more photons are absorbed than necessary to ionized the atom, the process is called above-threshold ionization and the free electron kinetic energy reads

$$
E_{k}=(n+s) \hbar \omega-E_{\text {ion }}
$$

where $\mathrm{n}$ is the necessary photon number for the electron ejection and $\mathrm{s}$ the additionally absorbed. The typical threshold intensity for this process to occur is above $10^{10} \mathrm{~W} \cdot \mathrm{cm}^{-2}$.

\section{Tunneling Ionization, $\gamma_{k}<1$}

For higher laser intensity, the laser electric field becomes strong enough to distort the atomic potential and considerably reduce the coulomb field felt by the electron. However, the coulomb barrier is still slightly above the binding energy of the electron but it may have a non-zero probability to tunnel through the potential barrier, and is called Tunneling Ionization.

Barrier Suppression Ionization, $\gamma_{k}<<1$

When the laser field is high enough $\left(\mathrm{I}>10^{14} \mathrm{~W} . \mathrm{cm}^{2}\right)$ to make the barrier fall during a fraction of the laser period, the electron can escape spontaneously. The laser intensity threshold necessary for this process is called the appearance intensity

$$
I_{\text {app }}=4 \times 10^{9}\left(\frac{E_{\text {ion }}^{4}[\mathrm{eV}]}{Z^{* 2}}\right)
$$

with $\mathrm{Z}^{*}$ the ionization state of the atom. Considering the hydrogen case, with $\mathrm{Z}=1$ and $E_{\text {ion }}=13.61 \mathrm{eV}$, the appearance intensity $I_{a p p} \approx 1.4 \times 10^{14} \mathrm{~W} \cdot \mathrm{cm}^{-2}$.

\subsubsection{Plasmas characteristic lengths}

Plasma can be defined by few characteristic lengths that play an important role in the characterization of the interaction within a plasma or between an external beam and a plasma. Depending on the considered scale length, different kinds of interactions between particles will happen, in particular due to the natural properties of quasi-neutrality of the plasma. Considering short spatial scales, collisional processes between particles dominate while collective plasma effects are the most relevant for larger scales. Finally, the scale lengths studied inside a plasma allow to estimate the state and the strength of the interaction between particle in a plasma. 


\section{Landau Length}

The Landau length represents the minimum approach distance between two particles. It corresponds to the distance where the mean kinetic particle energy is equal to the potential energy of interaction between the two particles. The Landau length between two electrons is expressed as following:

$$
\frac{1}{2} m_{e} v_{e}^{2}=k_{B} T_{e}=\frac{e^{2}}{4 \pi \epsilon_{0} r_{0}}
$$

with $m_{e}$ is the electron mass, $v_{e}$ the electron velocity, $k_{B}$ is the Boltzmann constant, $T_{e}$ the electron temperature and $r_{0}$ the Landau length given by:

$$
r_{0}=\frac{e^{2}}{4 \pi \epsilon_{0} k_{B} T_{e}} .
$$

The generalised Landau length between two particle $a$ and $b$ of charge $Z_{a}$ and $Z_{b}$ respectively is then given by:

$$
r_{a b}=\frac{Z_{a} Z_{b} e^{2}}{4 \pi \epsilon_{0} \mu v_{r e l}^{2}}
$$

with $\mu=\left(m_{a} m_{b}\right) /\left(m_{a}+m_{b}\right)$ the reduce mass and $v_{r e l}=\left|v_{a}-v_{b}\right|$ their relative speed.

\section{Debye Length and Shielding effect}

The Debye length is the characteristic screening distance of electrostatic phenomena in plasmas and defines the maximum distance where a collision can occur. To illustrate this physical significance, we consider a fixed ion with a charge $q_{i}$ in a plasma characterized by an uniform electron temperature $T_{e}=$ constant. The electrostatic potential $\Phi$ created by an ion with a charge $q_{i}=Z^{*} e$ is different than the Coulomb potential $\Phi_{\text {Coulb }}=q_{i} / 4 \pi \epsilon_{0} r$ since the electrons are attracted by the ion and in consequence screen its electric field. An elementary volume of electrons around the ion is then subjected to the electrical force

$$
\mathbf{f}_{\mathbf{E}}=n_{e} e \mathbf{E}=-n_{e} e \boldsymbol{\nabla} \Phi(r),
$$

and the pressure force resulting from the electron accumulation near the ion

$$
\mathbf{f}_{p e}=-k_{B} T_{e} \boldsymbol{\nabla} n_{e} .
$$

The resulting force in equilibrium gives

$$
\mathbf{f}_{\mathbf{E}}+\mathbf{f}_{p e}=-n_{e} e \boldsymbol{\nabla} \Phi(r)-k_{B} T_{e} \boldsymbol{\nabla} n_{e}=0 .
$$

Considering spherical symmetry (due to purely radial forces), we obtain:

$$
\frac{1}{n_{e}} \frac{d n_{e}}{d r}=-\frac{e}{k_{B} T_{e}} \frac{d \Phi}{d r} .
$$


The Boltzmann distribution of electrons density in this electrostatic potential can be obtain by integrating $(2.25)$

$$
n_{e}(r)=n_{e, 0} \exp \left(-\frac{e \Phi(r)}{k_{B} T_{e}}\right)
$$

with $n_{e, 0}$ the unperturbed electron density at a high enough distance from the ion. Considering a plasma where particles interact weakly (non-correlated plasma), meaning that the kinetic energy of electron is higher than the potential energy $e \Phi$, we can develop the term $\exp \left(e \Phi(r) / k_{B} T_{e}\right)$ in Taylor series and the electron distribution becomes

$$
n_{e}(r) \approx n_{e, 0}\left(1-\frac{e \Phi(r)}{k_{B} T_{e}}\right) .
$$

The ion electrostatic potential can be defined using the Maxwell-Poisson equation:

$$
\nabla . \mathrm{E}=-\Delta \Phi(r)=\frac{\rho}{\epsilon_{0}}=\frac{-e n_{e}+q_{i} n_{i, 0}}{\epsilon_{0}}
$$

Considering the hypothesis of "quasi-neutrality" $n_{e, 0} \approx Z^{*} n_{i, 0}$

$$
-\Delta \Phi(r)=\frac{-n_{e}+n_{e, 0}}{\epsilon_{0}} e
$$

By replacing (2.28) into (2.29), we find

$$
\Delta \Phi(r)=\frac{n_{e, 0} e^{2}}{\epsilon_{0} k_{B} T_{e}} \Phi(r)=\frac{\Phi(r)}{\lambda_{D}^{2}},
$$

where $\lambda_{D}$ is the Debye screening length and is written

$$
\lambda_{D}=\sqrt{\frac{\epsilon_{0} k_{B} T_{e}}{n_{e, 0} e^{2}}} .
$$

with the solution:

$$
\Phi(r)=\frac{q}{4 \pi \epsilon_{0} r} \exp \left(\frac{-r}{\lambda_{D}}\right) .
$$

The potential around the ion particle is represented in figure 2.6. It is worth mentioning that a condition for the existence of the plasma is that the dimension of the system $L_{\text {plasma }}$ should be larger than the Debye Length $\left(L_{\text {plasma }}>>\lambda_{D}\right)$. Thus, for distance $\mathrm{r}$ close to the particle $\left(\mathrm{r}<<\lambda_{D}\right)$, the potential felt around the particle is equal to the Coulomb potential. Considering equal or larger distances $\left(\mathrm{r} \geq \lambda_{D}\right)$ the exponential decreasing is dominant on the potential behaviour. The concept of Debye screening is thus only valid as long as the number of electron in the Debye sphere $N_{D}=\frac{4}{3} \pi n_{e, 0} \lambda_{D}^{3}$ is greater than unit $\left(N_{D}>>1\right.$.) 


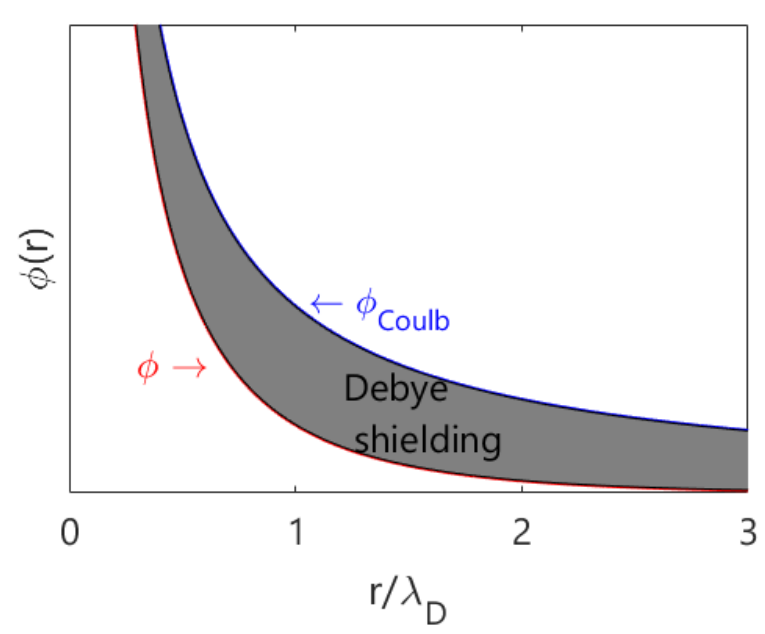

Figure 2.6: Potential $\Phi$ around an ion of charge $q_{i}$ in a plasma and Coulomb Potential $\Phi_{\text {Coulb }}$ according to the parameter $r / \lambda_{D}$ (with $\mathrm{r}$ the radial distance from the ion).

\subsubsection{Collective oscillations: the plasma Frequency}

The electronic plasma frequency is an important parameter which is essential for the stability of its macroscopic space charge neutrality. It corresponds to the inverse of the plasma response time to an electric perturbation. In order to calculate the plasma frequency $\omega_{p e}$, let's consider a mono-dimensional neutral and homogeneous $\left(n_{e}=\right.$ $Z^{*} n_{i}$ ) plasma at an initial time $t=0$. We introduce an electric field perturbation so that the electrons of the plasma, initially at the position $x_{0}$, are puled off to one direction from a distance equal to $\mathrm{x}$ while ions stay immobile (the inertia of the ions is such that their movement can be neglected). The electric field which results from this separation of charges tends to bring back the electrons towards their position of equilibrium and can be expressed as:

$$
E=-\frac{\sigma_{s}}{\epsilon_{0}}
$$

Where $\sigma_{s}$ is the surface charge density $\left(\sigma_{s}=-n_{e} e x\right)$. The equation of motion of the electrons subjected to the electric field perturbation $E$ is expressed according to Newton's second law $(\mathrm{F}=\mathrm{ma})$ with the force $F=-e E$

$$
\frac{d^{2} x}{d^{2} x}=-\frac{e E}{m_{e}}=-\frac{n_{e} e^{2} x}{m_{e} \epsilon_{0}}=-\omega_{p e}^{2} x,
$$

where

$$
\omega_{p e}=\sqrt{\frac{n_{e} e^{2}}{m_{e} \epsilon_{0}}},
$$

describes the angular frequency of this collective electron oscillations, also known as electron plasma frequency [radian/sec]. The rapid oscillations of the plasma density 
characterized by $\omega_{p e}$ are called Langmuir waves and correspond to the propagation of plasmons. Although the ion plasma frequency does not fulfill a very important role, we can define it as:

$$
\omega_{p i}=\sqrt{\frac{\left(Z^{*}\right)^{2} n_{i} e^{2}}{m_{i} \epsilon_{0}}}=\sqrt{\frac{Z^{*} m_{e}}{m_{i}}} \omega_{p e}<<\omega_{p e} .
$$

\subsubsection{Plasma parameters}

By using the relation between scale-length previously introduced, the plasma status can be characterized. Thus, several parameters are defined to describe the plasma state. At first approximation, two parameters can be considered, the plasma coupling parameter $\Gamma$ and the degeneracy parameter $\Theta$.

\section{Coupling Parameters}

The plasma behavior will be different depending on whether the effects of interactions between particles dominate or not. The strength of electron-electron correlations in plasma can be quantified by the so-called plasma coupling parameter $\Gamma$. It is defined by the ratio between the average Coulomb potential energy, between an electron and his closest neighborhood of the same nature $\left\langle E_{\text {Coulb,e }}\right\rangle=e^{2} / 4 \pi \epsilon_{0} a_{e}$ ( $a_{e}$ is the mean distance between two plasma electrons, also known as the Wigner-Seitz radius), and the average electronic kinetic energy per particles $\left\langle E_{k}\right\rangle=k_{B} T_{e}$ :

$$
\Gamma=\frac{\left\langle E_{\text {Coulb,e }}\right\rangle}{\left\langle E_{k}\right\rangle}=\frac{e^{2}}{4 \pi \epsilon_{0} k_{B} T_{e} a} \quad \text { with } \quad a=\left(\frac{4 \pi n_{e}}{3}\right)^{-1 / 3}
$$

This parameter allows to distinguish:

- $\Gamma<<1$, when the kinetic energy of the electrons dominates over their potential energy making the trajectory of each particle little influence by the interactions with the other particles, which defines a weakly coupled and collisionless plasma dominated by long-range collective plasma effects. It is also called $k i$ netic plasma.

- Conversely, when $\Gamma \geq 1$, the potential energy dominates and the particle trajectories are strongly affected by the near neighbor electrostatic interactions. This behavior corresponds to strongly coupled systems also called correlated plasma.

It is worth mentioning that the parameter $\Gamma$ designated the correlation between electron $\Gamma_{e e}$. We can also express this parameter between two ions at the distance $a_{i}$ between each other:

$$
\Gamma_{i i}=\frac{\left\langle E_{\text {Coulb }, i}\right\rangle}{\left\langle E_{k}\right\rangle}=\frac{\left(Z^{*} e\right)^{2}}{4 \pi \epsilon_{0} k_{B} T_{e} a_{i}} \approx Z^{* 5 / 3} \Gamma_{e e} .
$$

We note that the given expression for $\Gamma$ is valid as long as the considered plasma is nondegenerate. 


\section{Degeneracy parameter}

The plasma degeneracy degree $\Theta$ evaluates the influence of the Pauli exclusion rule on the target electrons. It is defined by the ratio between the kinetic energy of the plasma electrons and their Fermi energy

$$
\Theta=\frac{k_{B} T_{e}}{E_{F}} .
$$

By using this parameter one can distinguish:

- $\Theta>>1$ Non degenerate plasma. The kinetic energy of the plasma electrons dominates over their Fermi energy. The plasma follows the classical MaxwellBoltzmann statistics.

- $\Theta \leq 1$ Degenerate plasmas. The Pauli exclusion effect cannot be ignored and the plasma follows Fermi-Dirac statistics. Thus the electron kinetic energy must be expressed $E_{k}=k_{B} T_{e}+E_{F}$.

\subsubsection{Electromagnetic interaction with plasma}

According to the electromagnetic theory, the interaction between an electromagnetic field and matter is described by the Maxwells equations (S.I units)

$$
\begin{array}{ll}
\nabla . \mathbf{B}=0, & \\
\nabla . \mathbf{E}=\frac{\rho}{\epsilon_{0}}, & \text { (Gauss's Law) } \\
\nabla \times \mathbf{E}=-\frac{\partial \mathrm{B}}{\partial t}, & \text { (Faraday's Law) } \\
\nabla \times \mathbf{B}=\mu_{0} \mathbf{j}+\left(\frac{1}{c^{2}} \frac{\partial \mathbf{E}}{\partial t}\right) . & \text { (Ampere's Law) }
\end{array}
$$

with $\mathbf{E}$ and $\mathbf{B}$ the electric and magnetic field respectively, $\rho$ the charge density, $\mu_{0}$ the vacuum permeability and $\mathbf{j}=-e n_{e} \mathbf{v}_{\mathbf{e}}$ the current density. Assuming that the electrons have a non-relativistic movement in the wave $\left(v_{e} / c<<1\right)$, thus neglecting the magnetic force before the electric force, the electron equation of motion reads

$$
m_{e} \frac{d \mathbf{v}_{\mathbf{e}}}{d t}=-e \mathbf{E}
$$

Taking (2.41) and (2.43), we obtain the following propagation equation of electromagnetic wave

$$
\nabla \times \nabla \times \mathbf{E}=\nabla(\nabla . \mathbf{E})-\Delta \mathbf{E}=-\mu_{0} \frac{\partial j}{\partial t}-\frac{1}{c^{2}} \frac{\partial^{2} E}{\partial t^{2}},
$$

and assuming plasma local neutrality $(\nabla \cdot \mathbf{E}=0)$, we have

$$
\Delta \mathbf{E}=\mu_{0} \frac{\partial \mathbf{j}}{\partial t}+\frac{1}{c^{2}} \frac{\partial^{2} \mathbf{E}}{\partial t^{2}} .
$$


Combining (2.44) with the propagation equation, one finds

$$
\Delta \mathbf{E}=\frac{\mu_{0} e^{2} n_{e}}{m_{e}} \mathbf{E}+\frac{1}{c^{2}} \frac{\partial^{2} \mathbf{E}}{\partial t^{2}} .
$$

The field of the laser wave is expressed in the form of an electromagnetic wave such $\mathbf{E}=\mathbf{E}_{\mathbf{0}} \sin \left(\mathbf{k} . \mathbf{r}+\omega_{L} t\right)$ with $k$ the wave number and $\omega_{L}$ the laser frequency. We can thus express equation of propagation for the electric field

$$
-k^{2} c^{2} \mathbf{E}=\omega_{p e}^{2} \mathbf{E}-\omega_{L}^{2} \mathbf{E},
$$

where $k^{2} c^{2}=\omega_{L}^{2}-\omega_{p e}^{2}$ is the dispersion relation of electromagnetic waves in a plasma. This condition means that the laser electromagnetic wave can propagate in the plasma when $\omega_{L} \geq \omega_{p e}$ (see Figure2.7). It can be also explained defining the limit plasma density for which the laser can not propagate beyond, called the critical density $n_{c}$ and reads

$$
n_{c}\left[m^{-3}\right]=\frac{4 \pi^{2} m_{e}}{\mu_{0} e^{2} \lambda_{L}^{2}} \approx \frac{1.1 \times 10^{27}}{\lambda_{L, \mu m}^{2}},
$$

with $\lambda_{L, \mu m}$ the laser wavelength in units of microns. Subsequently, when reaching $n_{c}$ the laser gets reflected of the plasma. However, a part of the laser field will penetrate as an evanescent wave into the plasma up to a characteristic length called the skin depth $l_{s} \approx c / w_{p e}$ where the laser electric field has dropped by a factor $1 / \mathrm{e}$. The critical density $n_{c}$ is an important parameter separating two different regimes for electron acceleration to two ranges of densities. We can distinguish the under-dense matter regime for $n_{e}<n_{c}$ (gas target) where the propagation inside the plasma is possible. This may produce relevant effects as Laser Wakefield Acceleration process occurs (this process will not be presented in this thesis but further informations can be found in [Tajima \& Dawson, 1979]). For interaction occurring at $n_{e}>n_{c}$ (solid target), the regime is qualified as over-dense matter regime, and allows processes such as Target Normal Sheath Acceleration to occur (see next chapter of the thesis).
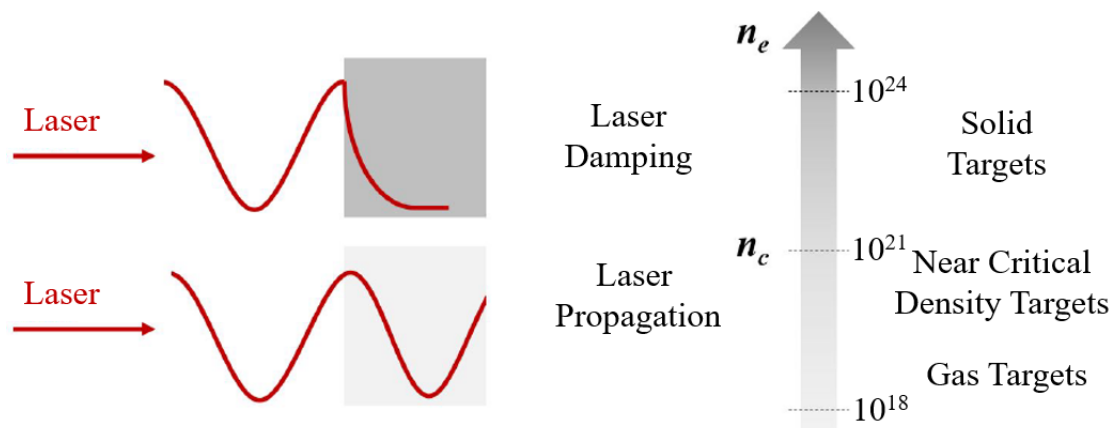

Figure 2.7: Two regimes of laser-matter interaction according to $n_{c}$. Illustration from [Zeraouli, 2020] 


\section{Chapter 3}

\section{Laser-Driven proton sources}

For more than two decades, laser-driven ion acceleration has been an important field of research with a potential interest for several applications, including in the biomedical area. Nowadays, available experimental laser intensities are in the order of $10^{22} \mathrm{~W} . \mathrm{cm}^{-2}$, which is at least two orders of magnitude below the threshold intensity for a direct transfer of energy between the laser and the ions. Hence, all current mechanisms of ion acceleration involve the electronic charge displacement and deep investigations are done to understand and determine the energy transfer from the electrons to the ions. However, the laser-matter interaction is a complex process which is still not totally controlled. The resulting interaction depends mainly on laser and plasma parameters such as laser irradiance, prepulse characteristic, target thickness, surface area... Up to now, number of different mechanisms have been identified theoretically and some of them have also been experimentally evidenced. In this chapter will be given some basic points of relevance about electron heating mechanisms and their transport in solid target, responsible of the charge separations and the acceleration of the ionic species. In the continuity, a brief overview of laserdriven proton beam generation and their transport in matter will be introduced to finally get into the main proton diagnostics used during the thesis study.

\subsection{State of the art}

Before the 2000s, high intensity laser matter interaction experiments provided ions energies up to few MeVs for different target such gas jet [Krushelnick et al., 1999] and thin solid foils [Beg et al., 1997]. However, the low brilliance and wide divergence of such ion emission made them unattractive for applications. Three main independent experiments done in 2000 [Clark et al., 2000; Maksimchuk et al., 2000; Snavely et al., 2000] recorded intense emission of multi-MeV proton up to $58 \mathrm{MeV}$ [Snavely et al., 2000] (see figure 3.1) from solid target of few $\mu \mathrm{m}$ thick, focusing laser beam to intensities $>10^{18} \mathrm{~W} . \mathrm{cm}^{-2}$, allowing to regain a strong interest in fundamental research and possible applications of ion acceleration. The protons were detected in forward direction with respect to the laser propagation, at the rear side of the target. It was 


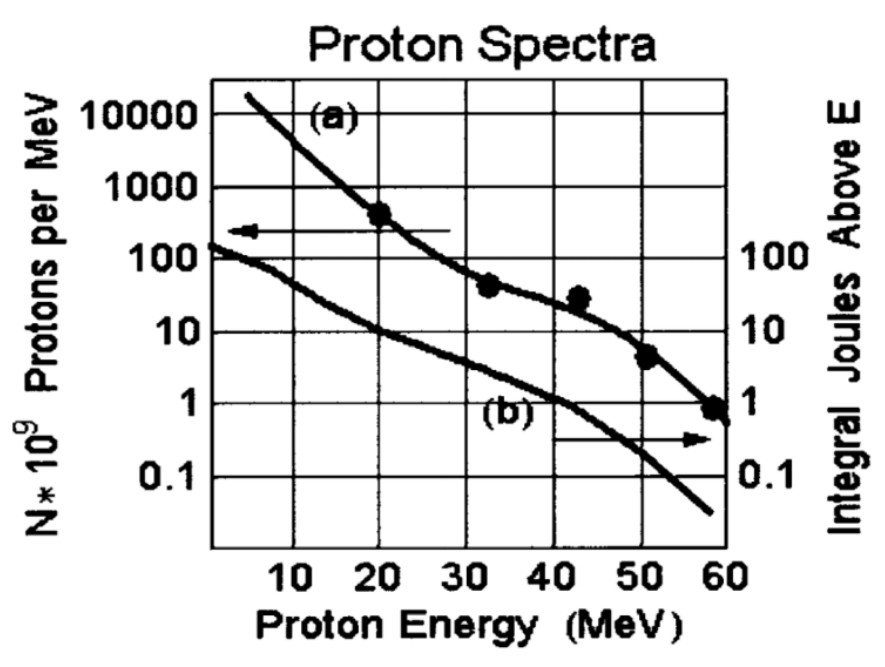

Figure 3.1: Proton energy spectrum obtain at the rear side of a $100 \mu \mathrm{m} \mathrm{CH}$ solid target irradiated by a laser energy of $423 \mathrm{~J}$ and pulse duration of $0.5 \mathrm{ps}$ at normal incidence. The laser intensity was about $3 \times 10^{20} \mathrm{~W} \cdot \mathrm{cm}^{-2}$. From [Snavely et al., 2000].

already confirmed from previous experiments that protons originated from impurities of thin layers of water or hydrocarbons which are ordinarily present on solid surfaces [Gitomer et al., 1986]. The common properties of these proton beams were the very large number of protons per shot (up to $\approx 10^{13}$ [Snavely et al., 2000]), a low emittance, picoseconds range source, showing a typical broadband energy spectrum up to a cut-off energy, combined with the strongly localized energy deposition at the end of their path, known as the Bragg Peak (see Figure 3.2). These properties make laseraccelerated protons and ions a favorable alternative to the conventional technology, since they might provide a more compact and economical particle source, and highly promising for any application requiring a localized energy deposition in dense matter which include (but not limited to), oncological hadrontherapy [Bulanov et al., 2002; Fourkal et al., 2002; Malka et al., 2004], radioisotope production for Positron Emission Tomography (PET) [Fritzler et al., 2003; Santala et al., 2001], warm dense matter production [Koenig et al., 2005], energy drivers for fast ignition inertial confinement fusion [Bychenkov et al., 2001; Roth et al., 2001; Temporal et al., 2002] or radiography of dense matter [Borghesi et al., 2003; Romagnani et al., 2005; Volpe et al., 2011]. However, a major requirement for several of these applications is an increase of the energy per nucleon up to hundreds with a control of the monochromacity and collimation of the ion beam. The laser beam stability and high repetition rate technology are also fundamental issues to develop in order to compete with the established technology of conventional accelerator. Strong efforts on the experimental and theoretical side have been done in the last decades to optimize and understand the physics behind this process and a number of different acceleration mechanisms have been evidenced 


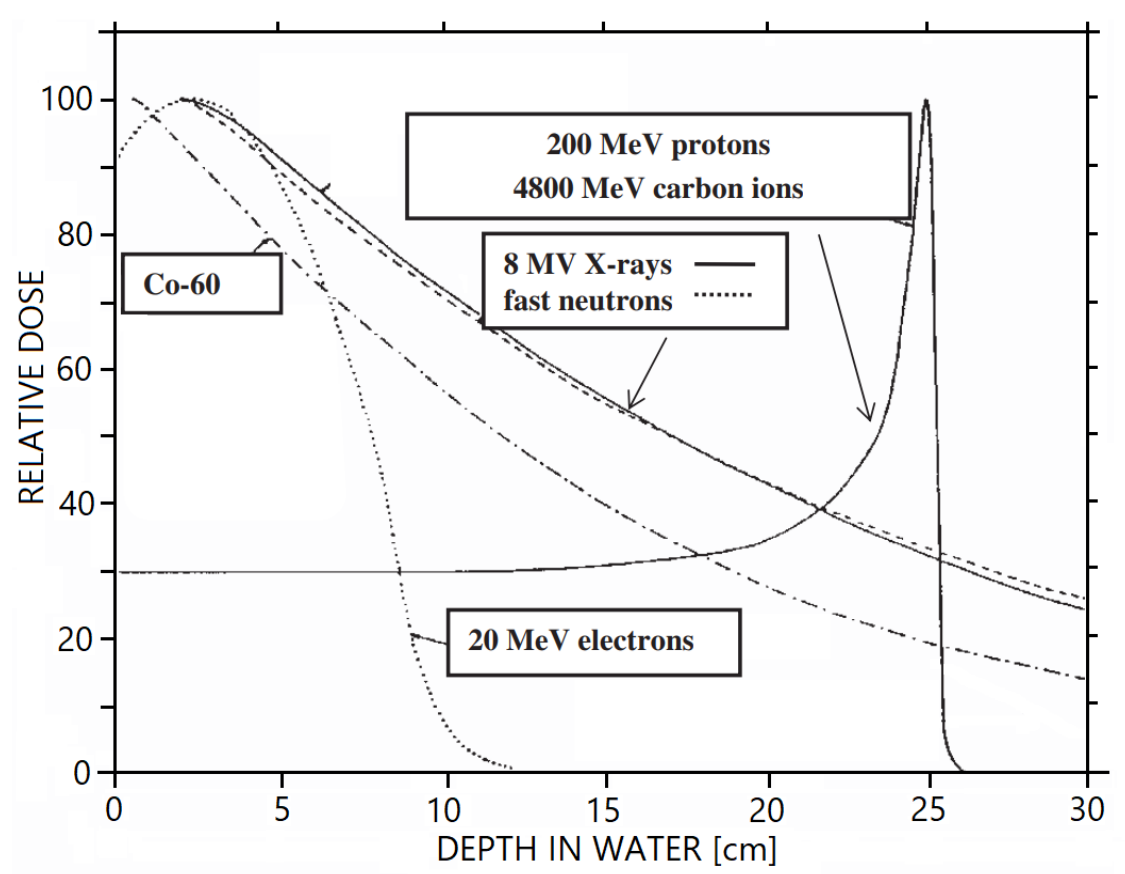

Figure 3.2: Depth dependence of the deposited dose for different radiations. From [Amaldi \& Kraft, 2005]

[Macchi et al., 2013] such as Target Normal Sheath Acceleration (TNSA), Radiation Pressure Acceleration (RPA), Break-Out After-burner (BOA) and Collisionless Shock Acceleration (CSA). In the framework of this thesis, the TNSA mechanism has been used and will be describe later in this chapter.

\subsection{Laser-driven fast electrons in solid target}

In the TNSA mechanism, protons are accelerated from the rear surface of the target due to the strong electric field generated by the high energy electrons (fast electrons) accelerated at the front surface, where the laser-plasma interaction occurs. Thus, the enhancement of the maximum energy and conversion efficiency of protons is strictly related to the mechanisms of fast electron acceleration and transport through the target. In this section will be introduced the most relevant coupling mechanisms between laser and dense plasma, related to the manuscript study. Indeed, several mechanisms participate in the absorption of the laser energy in the plasma, depending on the laser intensity, the pulse duration, polarization, angle of incidence, electron density and therefore bring together a large number of mechanisms participating in the electron acceleration and transport. 


\subsubsection{Single Electron Motion in an Ultra-High Intense Laser Pulse}

Due to the rapid development of ultra-intense lasers[Strickland \& Mourou, 1985], the relativistic threshold $I_{L} \lambda_{L}^{2} \gtrsim 10^{18} \mathrm{~W} . \mathrm{cm}^{-2} \cdot \mu \mathrm{m}^{2}$ of laser-plasma interaction is now easily reached in the laboratory, with intensities up to $10^{22} \mathrm{~W} . \mathrm{cm}^{-2}$. To introduce this regime, we begin with a description of the motion of a single electron placed in such a Ultra High-Intense (UHI) laser pulse propagating in vacuum along the x-axis direction.

The motion of an electron in the presence of this electromagnetic fields $(\mathbf{E}, \mathbf{B})$ is described by the Lorentz equation (CGS unit)

$$
\frac{d \mathbf{p}}{d t}=-e \mathbf{E}-e \frac{\mathbf{v}}{c} \times \mathbf{B},
$$

where $\mathbf{v}$ is the speed of the electron and $\mathbf{p}=\gamma m_{e} \mathbf{v}$ its relativistic momentum with its Lorentz factor

$$
\gamma=\frac{1}{\sqrt{1-v^{2} / c^{2}}}=\sqrt{1+\left(\mathbf{p} / m_{e} c\right)^{2}} .
$$

The first term on the right hand of (3.1) is the force that $\mathbf{E}$ applies on the electron and does not depend on its velocity, and is along the direction of $\mathbf{E}$. The second term is the force applied by $\mathbf{B}$ on the electron, proportional to the velocity of the charge and perpendicular to the $\mathbf{v}$ and $\mathbf{B}$. To make the equations in an invariant form, it is convenient to use the vector potential $\mathbf{A}$ in the Coulomb gauge condition $(\nabla \cdot \mathbf{A}=0)$

$$
\mathbf{A}=\left(0, \delta A_{0} \cos \phi, \sqrt{1-\delta^{2}} A_{0} \sin \phi\right)
$$

where $\phi=\frac{2 \pi}{\lambda_{L}}(c t-x)$ is the wave phase and $\delta$ is the polarization parameter such that $\delta=\{ \pm 1,0\}$ for a linearly polarized wave while $\delta= \pm 1 / \sqrt{2}$ is for circular one and other values correspond to elliptical polarization. Using the relation $\mathbf{E}=-\partial \mathbf{A} / \partial t$ and and $\mathbf{B}=c \nabla \times \mathbf{A}$ and the vectorial relation $\mathbf{v} \times \nabla \times \mathbf{A}=\nabla$, (3.1) can thus be expressed

$$
\frac{d \mathbf{p}}{d t}=e\left[\frac{\partial \mathbf{A}}{\partial t}+(\mathbf{v} \cdot \nabla) \mathbf{A}-\nabla(\mathbf{v} \cdot \mathbf{A})\right],
$$

and the kinetic energy conservation equation is written

$$
\frac{d}{d t}\left[(\gamma-1) m_{e} c^{2}\right]=\mathbf{v} \cdot \frac{d \mathbf{p}}{d t}=-e \mathbf{v} \cdot \mathbf{E}=e \mathbf{v} \cdot \frac{\partial \mathbf{A}}{\partial t} .
$$

Considering an electromagnetic plane-wave propagating in vacuum along the $\mathrm{x}$-axis, $\mathbf{A}$ is thus directed in the perpendicular plan yz with $\mathbf{A}=\mathbf{A}_{\perp}(x, t)$. The component of Equation (3.4) in the yz plan is given by:

$$
\frac{d \mathbf{p}_{\perp}}{d t}=e\left(\frac{\partial \mathbf{A}_{\perp}}{\partial t}+v_{\|} \cdot \frac{\partial \mathbf{A}_{\perp}}{\partial x}\right)
$$

After integrating (3.6) we get

$$
\mathbf{p}_{\perp}=e \mathbf{A}_{\perp}+\mathbf{p}_{\perp} 0=e \mathbf{A}_{\perp}
$$


where $\mathbf{p}_{\perp} 0$ is a constant representing the initial perpendicular impulsion of the electron an we consider $\mathbf{p}_{\perp} 0=0$ for an electron initially at rest in the laboratory frame. According to Equation (3.4), the electron movement along the propagation axis is given by

$$
\frac{d \mathbf{p}_{\|}}{d t}=-e\left(v_{\perp} \cdot \frac{\partial \mathbf{A}_{\perp}}{\partial x}\right)=\frac{-e}{\gamma m_{e}} \mathbf{p}_{\perp} \cdot \frac{\partial \mathbf{A}_{\perp}}{\partial x} .
$$

The perpendicular component of the electron impulsion (3.7) is conserved, allowing to write

$$
\frac{d \mathbf{p}_{\|}}{d t}=\frac{-e^{2}}{2 \gamma m_{e}} \frac{\partial \mathbf{A}_{\perp}^{2}}{\partial x}
$$

In the same way, (3.5) can be written

$$
\frac{d}{d t}\left[(\gamma-1) m_{e} c^{2}\right]=\frac{e^{2}}{\gamma m_{e}} \mathbf{A}_{\perp} \cdot \frac{\partial \mathbf{A}_{\perp}}{\partial t}=\frac{e^{2}}{2 \gamma m_{e}} \cdot \frac{\partial \mathbf{A}_{\perp}^{2}}{\partial t}
$$

and we can express an important relation

$$
\frac{d}{d t}\left((\gamma-1) m_{e} c^{2}-c \mathbf{p}_{\|}\right)=\frac{e^{2}}{\gamma m_{e}}\left(\frac{\partial}{\partial t}+c \frac{\partial}{\partial x}\right) \mathbf{A}_{\perp}^{2}=0,
$$

because the vector potential is only function of $\tau=t-x / c$. We thus obtain the conservation of the electron momentum for the parallel component

$$
\mathbf{p}_{\|}=(\gamma-1) m_{e} c .
$$

Considering the definition of the Lorentz factor $\gamma=\sqrt{1+\mathbf{p}_{\perp}^{2} / m_{e}^{2} c^{2}+\mathbf{p}_{\|}^{2} / m_{e}^{2} c^{2}}$, one can find the relation between the perpendicular and parallel component of the electron impulsion

$$
\mathbf{p}_{\|}=\frac{\mathbf{p}_{\perp}^{2}}{2 m_{e} c} \text { and } \gamma=1+\frac{1}{2}\left(\frac{e \mathbf{A}_{\perp}}{m_{e} c}\right)^{2} .
$$

The parallel impulsion is always positive, meaning that the electron is dragged along the wave propagation direction. Considering the two previous equations, we can expressed the angle $\theta$ of the electron movement according to the propagation axis of the wave

$$
\tan \theta=\frac{\mathbf{p}_{\perp}}{\mathbf{p}_{\|}}=\sqrt{\frac{2}{\gamma-1}} .
$$

Therefore, non-relativistic electrons are ejected from the wave along its polarization vector, while the relativistic electrons are ejected along the wave propagation direction. We can obtained the electron momentum using (3.7) and (3.12)

$$
\left\{\begin{array}{l}
\mathbf{p}_{\|}\left(p_{x}\right)=\frac{\left(e A_{0}\right)^{2}}{4 m_{e} c}\left[1+\left(2 \delta^{2}-1\right) \cos 2 \phi\right] \\
\mathbf{p}_{\perp}\left(p_{y}\right)=e A_{0} \quad[\delta \cos \phi] \\
\mathbf{p}_{\perp}\left(p_{z}\right)=e A_{0} \quad\left[\sqrt{\left(1-\delta^{2}\right)} \sin \phi\right] .
\end{array}\right.
$$


We note the derivative of the phase $\phi$ with respect to time $d \phi / d t=(\partial \phi / \partial t)+$ $\mathbf{v}_{\|}(\partial \phi / \partial x)=\omega_{L} / \gamma$ and the momentum variable $\mathbf{p}=\gamma m_{e}(d \mathbf{r} / d t)=\gamma m_{e}(d \phi / d t)(d \mathbf{r} / d \phi)=$ $2 \pi m_{e} c / \lambda_{L}(d \mathbf{r} / d \phi)$. We thus obtain the electron trajectory in the laboratory frame integrating (3.14)

$$
\left\{\begin{array}{l}
x=\frac{a_{0}^{2}}{4} \frac{\lambda_{L}}{2 \pi}\left[\phi+\frac{\left(2 \delta^{2}-1\right)}{2} \sin 2 \phi\right] \\
y=a_{0} \frac{\lambda_{L}}{2 \pi} \quad[\delta \sin \phi] \\
z=-a_{0} \frac{\lambda_{L}}{2 \pi}\left[\sqrt{\left(1-\delta^{2}\right)} \cos \phi\right]
\end{array}\right.
$$

with the dimensionless parameter $a_{0}=e A_{0} / m_{e} c=0.85 \times \sqrt{I_{L, 18} \lambda_{L, \mu \mathrm{m}}^{2}}$ where $I_{L, 18}$ is the laser intensity in units of $10^{18} \mathrm{~W} . \mathrm{cm}^{-2}$ and $\lambda_{L, \mu \mathrm{m}}$ is the laser wavelength in microns. These trajectories for linear and circular polarization are represented in Figure 3.4 for different values of $a_{0}$. Equation (3.15) shows that for a linearly polarized light, transversal oscillations are driven by $\omega_{L}$ and the electron motion in the propagation direction is split into a longitudinal oscillation at $2 \omega_{L}$ and a longitudinal drift term corresponding to the constant drift velocity

$$
\mathbf{v}_{\text {drift }}=\frac{\left\langle p_{x}\right\rangle}{\langle\gamma\rangle m_{e}}=\frac{a_{0}^{2} m_{e} c}{4\langle\gamma\rangle m_{e}}=\frac{a_{0}^{2} c}{4+a_{0}^{2}}
$$

Furthermore, we notice that in the case of circular polarization, $2 \delta^{2}-1=0$ and so $p_{x}=\left\langle p_{x}\right\rangle$ meaning that the high frequency component along the propagation direction vanishes. The trajectory is helicoidal and the transverse motion is circular with radius $\mathrm{r}=a_{0} / \sqrt{2} k=a_{0} \lambda_{L} /(2 \pi \sqrt{2})$ in the $y z$ plan. We now consider the average rest frame moving the drift velocity $\mathbf{v}_{\text {drift }}$, so $\left\langle p_{x}\right\rangle=0$. The frequency of electron oscillations in this frame is reduced due to the Doppler effect,

$$
\begin{aligned}
\omega_{L}^{\prime} & =\omega_{L} \sqrt{\frac{c-\mathbf{v}_{d r i f t}}{c+\mathbf{v}_{d r i f t}}}=\frac{\omega_{L}}{\sqrt{1+a_{0}^{2} / 2}} \\
\text { and } t_{L}^{\prime} & =t \sqrt{1+a_{0}^{2} / 2}
\end{aligned}
$$

so the phase is invariant $\phi^{\prime}=\phi$. By implementing these transformations in Equation 

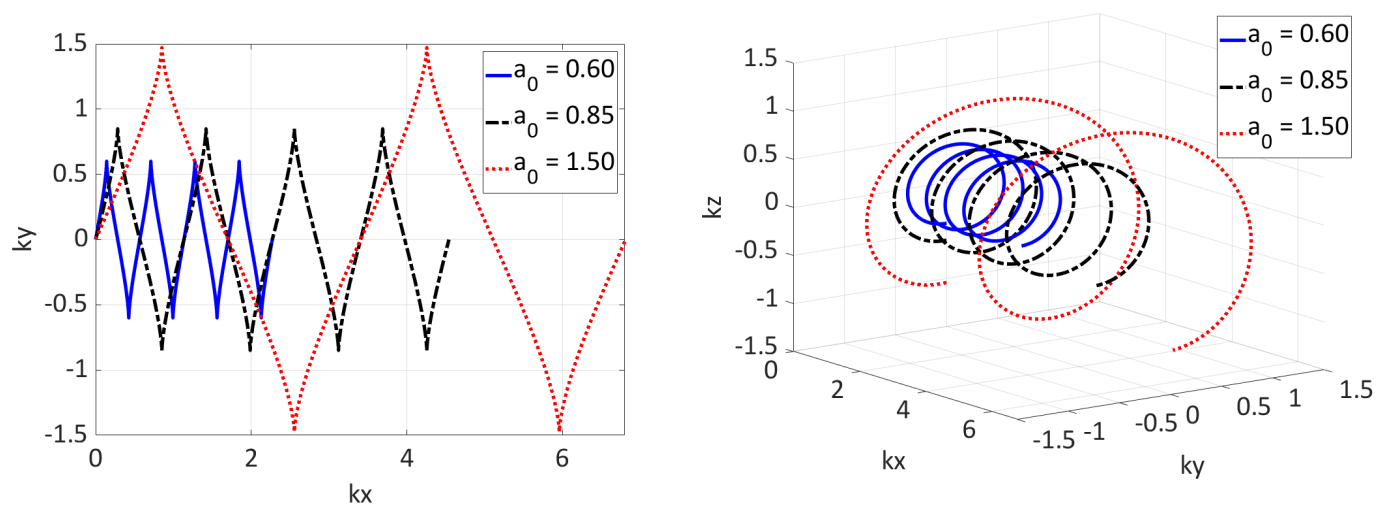

Figure 3.3: Electron trajectory in the laboratory frame under linear polarization (left) and circular polarization (right) for different values of the normalized laser amplitude $a_{0}: a_{0}=0.60$ (solid line), $a_{0}=0.85$ (dashed-dotted line) and $a_{0}=1.50$ (dotted line) according to Equations (3.15) corresponding to lasers intensities $I_{L}=$ $5.10^{17} \mathrm{~W} . \mathrm{cm}^{-2}, I_{L}=1.10^{18} \mathrm{~W} . \mathrm{cm}^{-2}$ and $I_{L}=3.10^{18} \mathrm{~W} . \mathrm{cm}^{-2}$ respectively considering an incident monochromatic laser pulse with a wavelength $\lambda_{L}=1 \mu \mathrm{m}$.

(3.15), one gives

$$
\left\{\begin{aligned}
x^{\prime} & =\frac{a_{0}^{2}}{4 \sqrt{1+a_{0}^{2} / 2}} \frac{\lambda_{L}^{\prime}}{2 \pi} \frac{\left(2 \delta^{2}-1\right)}{2} \sin 2 \phi \\
y^{\prime} & =\frac{a_{0}}{\sqrt{1+a_{0}^{2} / 2}} \frac{\lambda_{L}^{\prime}}{2 \pi}\left[\delta \sin \phi^{\prime}\right] \\
z^{\prime} & =-\frac{a_{0}}{\sqrt{1+a_{0}^{2} / 2}} \frac{\lambda_{L}^{\prime}}{2 \pi}\left[\sqrt{\left(1-\delta^{2}\right)} \cos \phi^{\prime}\right] .
\end{aligned}\right.
$$

In this frame, the longitudinal drift term vanishes and considering a linear polarization, the electron trajectory consists in oscillation $2 \omega_{L}$ in the $\mathrm{x}$ direction and $\omega_{L}$ in the perpendicular direction, describing a figure-of-eight curve (see Figure 3.4). For circular one, the orbit of the electron transforming to a reference frame moving with velocity $v_{x}=\mathbf{v}_{\text {drift }}$ is a closed circle in the $y z$ plan (see Figure 3.4). The study concerning single electron motion shows that electrons interacting with UHI laser plane wave can not acquire energy directly from the laser pulse. In the presence of the laser pulse wave, the electron immediately starts to drift with an average momentum $\left\langle p_{x}\right\rangle$ and stop moving immediately after the laser pulse ended. The fact that the electron does not gain any energy from the laser is the consequence of its adiabatic motion and is known as the Lawson-Woodward theorem [Lawson, 1979; Woodward, 1948]. Anyway, some processes of interaction can break this adiabaticity, where a fraction of the laser energy can be transferred to the electrons of a plasma. In the next section, we will get into the acceleration mechanisms of electrons in solid materials, where 

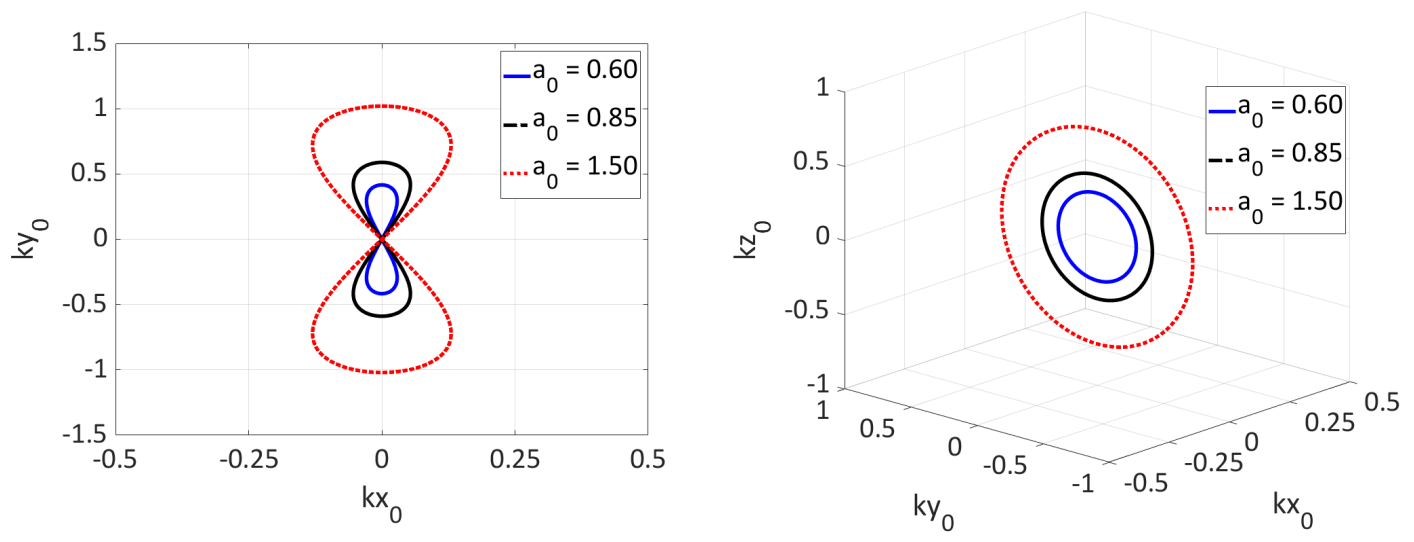

Figure 3.4: Electron trajectory in the rest frame where there is no average drift along the propagation direction (x) under linear polarization (left) and circular polarization (right) for different values of the normalized laser amplitude $a_{0}: a_{0}=0.60$ (solid line), $a_{0}=0.85$ (dashed-dotted line) and $a_{0}=1.50$ (dotted line) according to Equations (3.15) corresponding to lasers intensities $I_{L}=5.10^{17} \mathrm{~W} . \mathrm{cm}^{-2}, I_{L}=1.10^{18} \mathrm{~W} . \mathrm{cm}^{-2}$ and $I_{L}=3.10^{18} \mathrm{~W} \cdot \mathrm{cm}^{-2}$ respectively considering an incident monochromatic laser pulse with a wavelength $\lambda_{L}=1 \mu \mathrm{m}$.

these processes take place.

\subsubsection{Ponderomotive Force}

We saw previously that in a monochromatic plane wave, the coupling between the E-field and the B-field make the particle to drift in the direction of propagation while quivering in the oscillating electric field. However, in the case of a laser pulse, the field amplitude of the electromagnetic wave is not constant with a finite width and duration making appear a force term called ponderomotive force, directed along the intensity gradient. This force is a nonlinear force and rises as a function of the spatial and temporal profile of laser intensity [Mulser \& Bauer, 1988]. We will first introduce the expression of the ponderomotive force acting on a single electron in a non-relativistic case.

For a incident pulse linearly polarized propagating in the x-direction characterized by an electric Field $\mathbf{E}_{\mathbf{1}}(\mathbf{r}, t)=E_{1}(r, t) \cos \left(\omega_{L} t-k x\right) \mathbf{e y}$ and a magnetic field $\mathbf{B}_{\mathbf{1}}(\mathbf{r}, t)$ (we consider only the high frequency electromagnetic field). The electron equation movement reads (CGS unit)

$$
m_{e} \frac{d \mathbf{v}}{d t}=-e \mathbf{E}_{\mathbf{1}}(\mathbf{r}, t)-e \frac{\mathbf{v}}{c} \times \mathbf{B}_{\mathbf{1}}(\mathbf{r}, t),
$$

Considering the first order term in the perturbation expansion, the electron oscillate along the laser electric filed direction at the laser frequency $\omega_{L}$ around the non 
perturbed position $\mathbf{r}_{0}$. The equation reads

$$
m_{e} \frac{d \mathbf{v}_{1}}{d t}=-e \mathbf{E}_{\mathbf{1}}\left(\mathbf{r}_{0}, t\right)
$$

with the trajectory

$$
\mathbf{r}_{1}=\frac{e}{m_{e} \omega_{L}^{2}} \mathbf{E}_{\mathbf{1}}\left(\mathbf{r}_{0}, t\right) .
$$

If we consider the second order perturbation term, the electron movement reads:

$$
m_{e} \frac{d \mathbf{v}_{2}}{d t}=-e\left(\mathbf{r}_{1} . \nabla\right) \mathbf{E}_{\mathbf{1}}\left(\mathbf{r}_{0}, t\right)-e \frac{\mathbf{v}_{1}}{c} \times \mathbf{B}_{\mathbf{1}}\left(\mathbf{r}_{0}, t\right)
$$

where the first term take into account the electron movement in the electric field and the second term the magnetic part of the Lorentz force. By injecting the Equation (3.21) into (3.22) and using the Maxwell-Faraday Equation (2.42), one obtain

$$
m_{e} \frac{d \mathbf{v}_{2}}{d t}=-\frac{e^{2}}{m_{e} \omega_{L}^{2}}\left[\left(\mathbf{E}_{\mathbf{1}} \cdot \nabla\right) \mathbf{E}_{\mathbf{1}}-\omega_{L} \mathbf{E}_{\mathbf{1}} \times \int^{t}\left(\nabla \times \mathbf{E}_{\mathbf{1}}\right) d t\right] .
$$

Using the fonction $\nabla(\mathbf{A} . \mathbf{B})=(\mathbf{B} . \nabla) \mathbf{A}+(\mathbf{A} \cdot \nabla) \mathbf{B}+\mathbf{B} \times \nabla \times \mathbf{A}+\mathbf{A} \times \nabla \times \mathbf{B}$ and averaging the force applied on the electron over a laser period, one find

$$
\begin{aligned}
\left\langle m_{e} \frac{d \mathbf{v}_{2}}{d t}\right\rangle & =-\frac{e^{2}}{m_{e} \omega_{L}^{2}}\left\langle\left(\mathbf{E}_{\mathbf{1}} \cdot \nabla\right) \mathbf{E}_{\mathbf{1}}+\mathbf{E}_{\mathbf{1}} \times \nabla \times \mathbf{E}_{\mathbf{1}}\right\rangle \\
& =-\frac{e^{2}}{2 m_{e} \omega_{L}^{2}} \nabla\left\langle\mathbf{E}_{\mathbf{1}}^{2}\right\rangle \\
& =-\frac{e^{2}}{4 m_{e} \omega_{L}^{2}} \nabla\left\langle\left[\left|E_{1}^{2}\right|+\left|E_{1}^{2}\right| \cos \left(2 \omega_{L} t-k x\right)\right]\right\rangle
\end{aligned}
$$

The ponderomotive force is usually defined by the average part of this force and it reads

$$
\left\langle\mathbf{F}_{\mathbf{p}}\right\rangle=-\frac{e^{2}}{4 m_{e} \omega_{L}^{2}} \nabla\left|E_{1}^{2}\right| .
$$

Noting the laser intensity $I_{L}(r, t)=c \epsilon_{0} E_{1}(r, t)^{2} / 2$, the mean ponderomotive force over a laser period can be written:

$$
\left\langle\mathbf{F}_{\mathbf{p}}\right\rangle=-\frac{e^{2}}{2 m_{e} \omega_{L}^{2}} \frac{1}{c \epsilon_{0}} \nabla I_{L} .
$$

Consequently, the ponderomotive force tends to eject plasma electrons from the focal spot center where the laser intensity is highest. However this force is inverse proportional to the particle mass, so its direct effect on ion trajectories is small. Nevertheless, it acts on ions via electrons by generating an electrostatic field due to charge separation. The expression of the ponderomotive force has been generalized for relativistic intensities and reads [Quesnel \& Mora, 1998]

$$
\left\langle\mathbf{F}_{\mathbf{p}}\right\rangle=-m_{e} c^{2} \nabla\langle\gamma\rangle \text {. }
$$

It is worth mentioning that the final electron energy is given by $\Delta U=\left(m_{e} c^{2}(\gamma-1)\right)$. 


\subsubsection{Heating Mechanisms}

Current knowledge on fast electron generation is still limited by the complexity of the physics involved, and therefore possibly not totally understood yet. Indeed, several processes are responsible for the intense laser pulse absorption and resulting electron heating, mainly depending on the laser intensity regime, plasma properties as well as laser incidence angle. However, in experimental case, a mixed contribution from each mechanism takes place, thus leading to complex processes difficult to estimate. As reported previously, a condition for electron heating is the break of adiabaticity of the electron motion in the laser field. Depending on the laser parameters or the geometry of the interaction, various processes exist. In the following, the most important absorption mechanisms in overdense plasma are introduced.

\section{Collisional Mechanisms}

In classical interaction regime with low laser intensities $I_{L}<10^{15} \mathrm{~W} \cdot \mathrm{cm}^{2}$, the largest contribution to absorption is due to electron-ion collisions known as the inverse bremsstrahlung with reference to the quantum image where a photon is absorbed by an electron scattering on an ion, which is the inverse of the bremsstrahlung process. Electromagnetic wave is thus damped by the plasma and the electron oscillation energy is converted in thermal energy, heating up the plasma. The absorption coefficient reads [Rozmus et al., 1996]

$$
\eta_{a b s}=\frac{\omega_{L}}{\omega_{p e}} \sqrt{\frac{8 \nu_{e i}}{\omega_{L}}}
$$

where $\nu_{e i}$ is the frequency of these electron-ion collisions given by [Kruer, 1988]

$$
\nu_{e i}=\frac{4 \sqrt{2 \pi}}{3} \frac{n_{e} Z^{*} e^{4}}{m_{e}^{2} v_{T, e}^{3}} \ln \Lambda_{e i} \approx 3 \times 10^{-6} \frac{Z^{*} n_{e} \ln \Lambda_{e i}}{T[k e V]^{3 / 2}},
$$

with $\ln \Lambda_{e i}$ the Coulomb logarithm corresponding to the factor by which small-angle collisions are more effective than large-angle collisions in scattering process. However, we can see from Equation (3.29) that the collision frequency decreases for higher electron temperature and other mechanism of collisionless absorption start to dominant.

\section{Resonant Absorption}

At oblique incidence, the position where an electromagnetic wave is reflected is no longer at the critical surface $x_{c}$ but at the position where the density $n_{e}=n_{c} \cos ^{2} \theta<n_{c}$ according to Snell's law. When the laser is p-polarized i.e with electric field in the plane of incidence, a fraction of the laser field is able to cross this region until reaching the critical density and thus excites the electron along the density gradient $L_{\text {grad }}$ region. When the laser frequency is equal to the plasma frequency $\omega_{L}=\omega_{p e}$, which means $n_{e}=n_{c}$ the effect is maximized and the plasma enter in resonance with the electromagnetic wave. The phenomenon is called Resonant Absorption [Forslund et al., 1975; Freidberg et al., 1972]. Figure 3.5 illustrates this geometry of incidence. In 


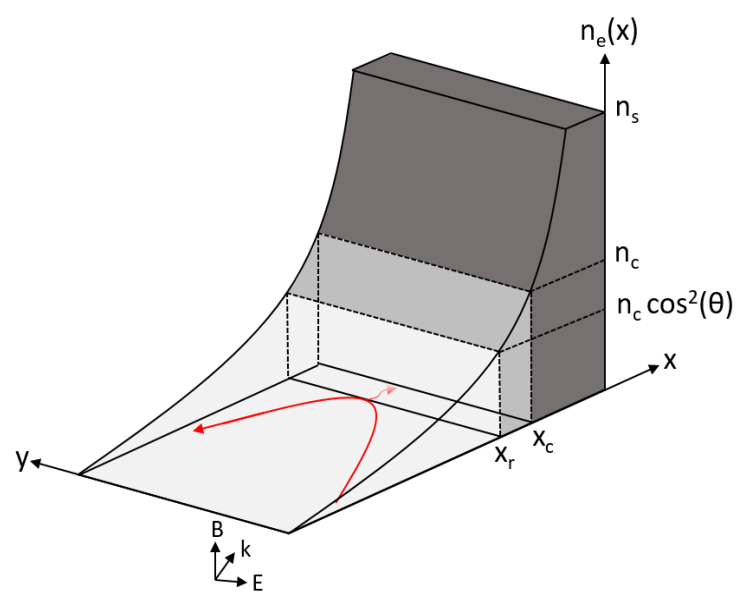

Figure 3.5: Representation of a p-polarized ( $\mathrm{x}-\mathrm{y}$ plane of incidence) electromagnetic wave interacting with a target in oblique incidence. The wave is reflected with an angle $\theta$ on the plasma gradient $L_{\text {grad }}$. At the position $x_{r}$ corresponding to a plasma density $n_{c} \cos ^{2} \theta$ an evanescent wave is created and excites the plasma wave, entering in resonance at the critical density $n_{c}$.

classical regime, and for a relative long density scale-length, the fraction of absorbed laser energy $\eta_{a b s}$ is define by [Gibbon, 2005]

$$
\eta_{a b s} \approx \frac{1}{2}\left[2.3\left(k L_{\text {grad }}\right)^{1 / 3} \sin \theta \exp \left(\frac{2\left(k L_{\text {grad }}\right)}{3}\right)\right]^{2}
$$

with $k$ the wave number and shows a maximum absorption for $\theta_{\max } \approx \arcsin \left(1 / k L_{\text {grad }}\right)^{1 / 3}$. However, this mechanism is falling for short gradient length. The population of electrons accelerated by this mechanism can be describe by a Maxwellian distribution, with a temperature [Forslund et al., 1977]

$$
T_{r a}[k e V] \approx 10\left(I_{16} \lambda_{L, \mu \mathrm{m}}^{2} T_{e}\right)^{(1 / 3)}
$$

with $T_{e}$ the plasma background electron temperature in $\mathrm{keV}, I_{16}$ is the laser intensity in units of $10^{16} \mathrm{~W} . \mathrm{cm}^{2}$ This process dominates to intensities up to $10^{17} \mathrm{Wcm}^{2}$. At higher intensities other mechanisms are mainly responsible of the laser energy absorption. Resonance absorption can still contribute to the total absorption but will not be the dominant factor. 


\section{Vacuum Heating}

When the plasma has a very steep density gradient $L_{\text {grad }}$ the resonant absorption ceases to work. This mechanism, called Vacuum Heating was first discovered by Brunel in 1987 [Brunel, 1987]. He predicted a transition of the resonant absorption depending on the density gradient length of the plasma toward a new mechanism "not so resonant" called the Brunel absorption. Indeed, when electrons undergo oscillations along the density gradient with an amplitude $x_{p} \approx e E_{L} / m_{e} \omega_{L}=v_{o s c} / \omega_{L}$, the resonance will breaks down if this amplitude exceeds the density scale length $L_{\text {grad }}$, which means if $v_{\text {osc }} / \omega_{L}>L_{\text {grad }}$.

Let's consider a p-polarized laser with an oblique incidence on the plasma front surface. In the first half of the laser cycle, the electrons from plasma-vacuum interface (the skin length) experiencing the longitudinal component of the laser field are pulled out in vacuum leaving the target surface with a positive charge. An electrostatic electric field is thus created due to the separation of charge. During the second half of the laser cycle, as the total electric field reverses (laser field combined with the electrostatic field of charge separation) the electrons are accelerated back into the plasma. Because the plasma is overdense, the electromagnetic wave vanishes beyond the skin length $l_{s}$ and the electrons are shielded from the laser field, allowing them to spread deeper into the plasma with the kinetic energy acquired from the electrostatic field where they eventually dissipate their energy through collisions.

An estimation of laser energy absorption is [Gibbon, 2005]:

$$
\eta_{a b s} \approx \frac{1}{\pi a_{0}} f\left[\sqrt{1+f^{2} a_{0}^{2} \sin (\theta)^{2}-1}\right] \frac{\sin (\theta)}{\cos (\theta)},
$$

where $f=1+\sqrt{1-\left(4 a_{0} \sin (\theta)^{3} / \pi \cos (\theta)\right)}$. Finally, for small gradient length such as $L_{\text {grad }}<0.1 \lambda_{L}$ and intensities in the range $10^{16}-10^{18}$, the vacuum heating becomes the dominant electron heating mechanism.

\section{Relativistic J x B Heating}

In the relativistic regime $\left(I_{L} \lambda_{L}^{2} \gtrsim 10^{18} \mathrm{~W} \cdot \mathrm{cm}^{2} \cdot \mu \mathrm{m}^{-2}\right)$, a similar effect to vacuum heating on a steep density gradient is responsible of the electron acceleration. Contrary to the vacuum heating, the influence of the magnetic field of the laser pulse is not neglected and the heating mechanism is driven by the $(\mathbf{v} / c) \times \mathbf{B}$ term of the Lorentz force which oscillates at twice the laser frequency. This mechanism is called the $\mathbf{J} \times \mathbf{B}$ heating and was first introduced by Kruer and Estabrook in 1985 [Kruer \& Estabrook, 1985]. In the case of a linear polarization, we can distinguish 2 terms from Equation (3.24). The first term describes the electron acceleration along the laser propagation axis while the high frequency component oscillating at $2 \omega_{L}$ can lead to laser energy absorption, responsible for the so-called $\mathbf{J} \times \mathbf{B}$ heating. When electrons are ejected in vacuum by the Lorentz force from regions of higher intensity, it creates a strong electrostatic field due to the charge separation. When the electric field reverse, electrons are then recalled into the overdense plasma with an amplification due to the force generated by the electrostatic field. The adiabaticity of the electron motion is 
then broken when they are crossing the plasma skin layer, making them to escape the region from the laser solid interaction zone thanks to their residual energy. This acceleration mechanism has been confirmed by Wilks [Wilks et al., 1992] and expressed the mean kinetic energy also called the temperature of the accelerated electrons.

$$
\begin{gathered}
T_{\mathbf{J} \times \mathbf{B}} \approx m_{e} c^{2}\left(\sqrt{1+a_{0}^{2}}-1\right)=m_{e} c^{2}\left(\sqrt{1+\frac{I_{L, 18} \lambda_{L, \mu \mathrm{m}}^{2}}{1.37}}-1\right), \\
T_{\mathbf{J} \times \mathbf{B}}[k e V] \approx 511\left(\sqrt{1+\frac{I_{L, 18} \lambda_{L, \mu \mathrm{m}}^{2}}{1.37}}-1\right) .
\end{gathered}
$$

where the laser intensity $I_{L, 18}$ is expressed in the $10^{18}$ W.cm ${ }^{-2}$.

\section{Discussion on heating mechanisms}

A large number of mechanisms are responsible for the electron acceleration, depending mainly in the density gradient, the laser intensity and the incidence angle. Considering a non-relativistic laser intensity with an oblique incidence on the target, the resonant absorption and the vacuum heating mechanisms are the main responsible for the electron acceleration, according to the density gradient length. By increasing the laser intensity, the $\mathbf{J} \times \mathbf{B}$ heating starts to dominate progressively for steep density gradient. Let's note that in the case of long density gradients characterized by long underdense plasma generated by the ASE pedestal, other non-linear mechanism called parametric instabilities [Drake et al., 1974] can be responsible for the electron acceleration. Finally, one can cite the stochastic heating [Chopineau et al., 2019; Mendonça \& Doveil, 1982] when electrons interact with the stationary wave corresponding to the superposition of an incident electromagnetic wave and a backscattered electromagnetic wave, their motion can become chaotic, meaning that small differences in the initial conditions produce a large divergence in the electron trajectory with time. This mechanism can contribute to the acceleration of high energy electrons. However, in the practice, the situation is more complex because all contribution are mixed.

The measurement of energy distribution of the fast electron generated in the dense target is not trivial. Placing an electron spectrometer into the target chamber for a direct measurement of the fast electron population escaping the target will not give a representative population number. Indeed, due to the strong electrostatic field present at the rear side of the target, most part of them are re-injected into the target and can not escape while only the fastest electrons (few MeV) are able to escape. This mechanism is known as electron refluxing [Neumayer et al., 2010]. An indirect method is performed to measure the fast electron distribution which consists in measuring the x-ray emission, either Bremsstrahlung and/or K- $\alpha$ photon generated by the fast electrons crossing the target. A Maxwell-Jüttner distribution function is 
commonly used to describe the generated electron energy spectra

$$
f(E) \propto \beta_{b} \gamma^{2} \exp \left[\frac{E}{T_{h}}\right]
$$

with $E=m_{e} c^{2}(\gamma-1)$ the hot electron energy, $\beta_{b}=v_{b} / c$ the normalized beam electron velocity and $T_{h}$ the averaged hot electron energy. A two temperature behavior model from [Sawada et al., 2014] is also used for simulations to express the energy distribution function

$$
f_{\text {total }}(E) \propto(1-R) \times f_{h 1}\left(E, T_{h 1}\right)+R \times f_{h 2}\left(E, T_{h 2}\right)
$$

with $T_{h 1}$ and $T_{h 2}$ the coldest and hottest temperature components of the fast electron beam, respectively, $R$ is a scale factor for the hot component and

$$
f(E, T) \propto \frac{1}{k_{B} T} \exp \left(-\frac{E}{k_{B} T}\right) .
$$

Many studies have been done to measure the fast electron temperature according to the laser intensity $I_{L}$ or the parameter $I_{L} \lambda_{L}^{2}$. For example, by measuring the bremsstrahlung spectra of fast electrons generated at intensities $\leq 10^{19} \mathrm{~W} . \mathrm{cm}^{2},[\mathrm{Beg}$ et al., 1997] propose the following empirical expression

$$
T_{h}^{[\mathrm{Beg}]}[k e V]=215\left(I_{L, 18} \lambda_{L, \mu \mathrm{m}}^{2}\right)^{(1 / 3)}
$$

Another scaling law was introduced by [Wilks et al., 1992], for higher intensities such as

$$
\begin{aligned}
10^{18} \mathrm{~W} \cdot \mathrm{cm}^{2} \leq I_{L} \leq & 10^{21} \mathrm{~W} \cdot \mathrm{cm}^{2} \\
& T_{h}^{[\mathrm{Wilks}]}[\mathrm{keV}]=511\left(\sqrt{1+0.73 I_{L, 18} \lambda_{L, \mu \mathrm{m}}^{2}}-1\right)
\end{aligned}
$$

The figure 3.6 shows the evolution of the fast electron temperature with the laser intensity for Beg and Wilks high temperature electron scaling laws.

The characterization of the quantity of laser energy converted into fast electrons called conversion efficiency $\eta_{L}$, has been extensively measured in various experiments in the last decades. However, the conversion efficiency is strongly dependent on the absorption mechanism i.e laser intensity, laser contrast, laser beam incidence angle, etc...making it a very complex quantity to obtain, due to the complexity of hot electron generation dynamics and still remains an open question for the laser-plasma physic community. However, the work of [Davies, 2008] and [Town et al., 2005] about 


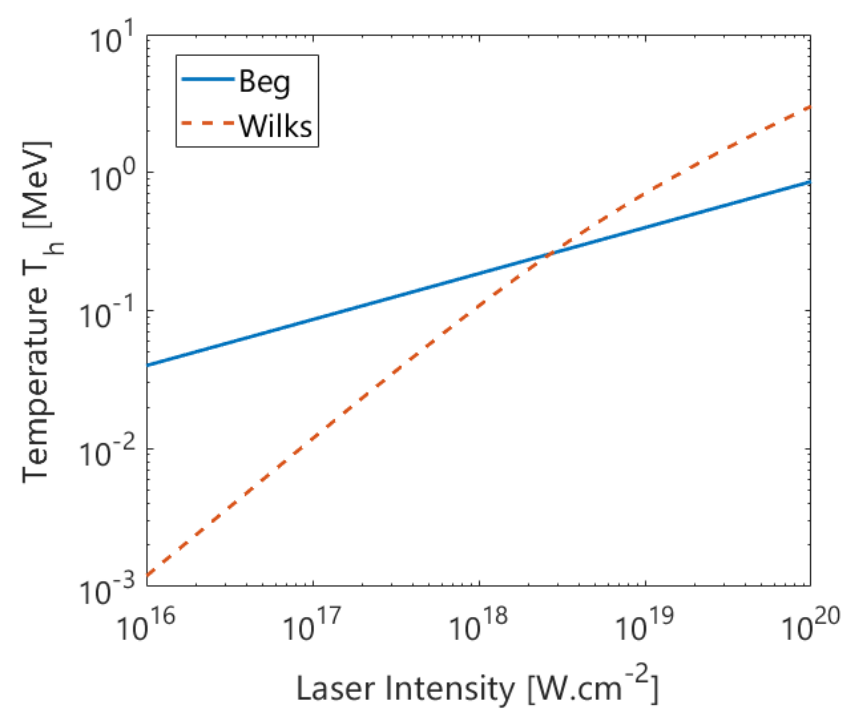

Figure 3.6: Comparison between both scaling laws for hot electron temperature.

the laser absorption by overdense plasmas in relativistic regime for laser intensity $I_{L}=2 \times 10^{18}$ to $2 \times 10^{20} \mathrm{~W} . \mathrm{cm}^{-2} \mu \mathrm{m}^{2}$ give a good dependence of $\eta_{L}$

$$
\eta_{L}=\left(2.3 \times 10^{-22} I_{L} \lambda_{L, \mu m}^{2}\right)^{0.2661} .
$$

Another important feature, in the framework of fast electron acceleration, is the divergence of the hot electron beam and is crucial to establish the electron density which characterizes the Debye sheath and thus the transverse dimension of the ion beam. It is however quite complicated to calculate theoretically the fast electron divergence. Several experiments were performed to characterize it such as [Green et al., 2008] which shows an increase of divergence according to the laser intensity on target (see figure 3.7).

\subsubsection{Fast electron beam transport}

During the propagation of the electron beam in a dense material, various effects affect their spread, which we classify generally according to their collisional (binary) or collective aspects. In this section, a quick and non-exhaustive description of the transport mechanisms is given. A presentation of a more detailed study of the complex collective and collisional transport theory can be found in the $\mathrm{PhD}$ thesis of [Gremillet, 2001].

\subsubsection{Collisional effects}

The high density medium requires taking into account elastic and inelastic collisions of electrons. Elastic collisions happen in an electron-ion collision. Due to their 


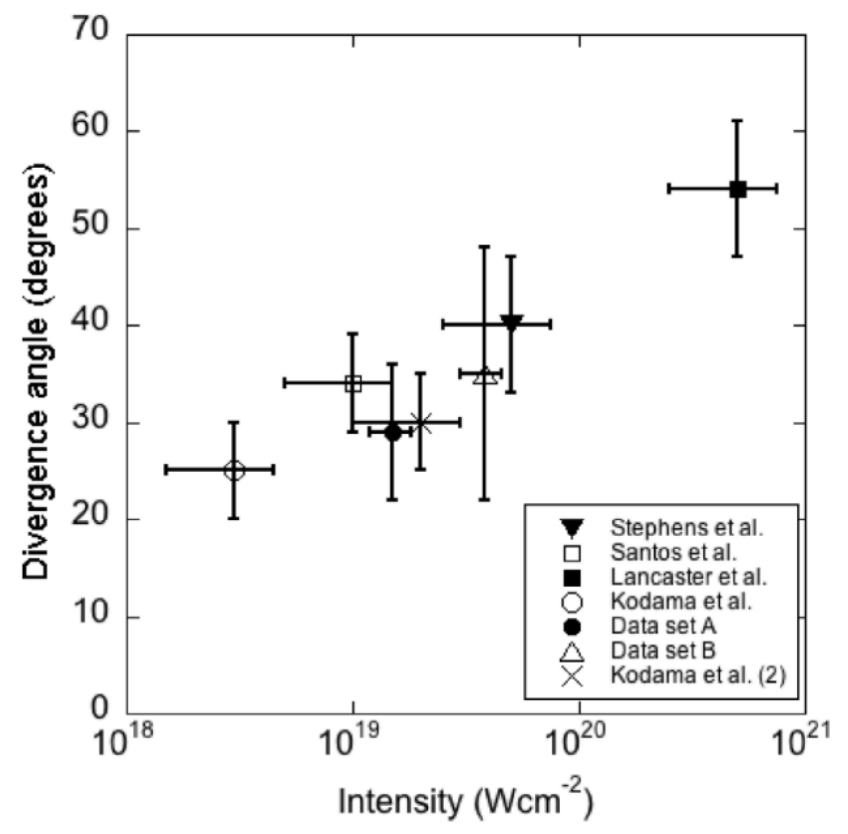

Figure 3.7: Measured electron beam divergences as a function of laser intensity on target. Taken from [Green et al., 2008].

huge mass, ions will absorb a negligible fraction of energy from the electron and the electron will be thus slightly deflected with almost the same initial energy.

\section{Angular scattering}

Considering a classical regime, the description of the collision with an immobile ion without internal structure, generating a Coulombian potential, can be estimated by using the cross section from Rutherford [Rutherford, 1911]

$$
\frac{d \sigma_{R}}{d \Omega}=4 r_{e}^{2} \frac{Z^{2}}{\beta^{4}}\left(\frac{1}{4 \sin ^{2}(\theta / 2)}\right)^{2}
$$

with $\mathrm{Z}$ the atomic number of the medium, $r_{e}=e^{2} /\left(4 \pi \epsilon_{0} m_{e} c^{2}\right)$ the electron classical radius and $\theta$ the deflection angle. One can see that the diffusion will be very effective for low energy electrons in a solid at high $\mathrm{Z}$ and that small angle deviation are consequently dominating over large angle deviations. A singularity exists at small angle, due to long range character of the Coulomb potential. To correct it, one has to take into account the screening effect by atomic electrons (in neutral material) or by free electrons (in a plasma) on the coulomb potential. Finally the potential decreases faster than the Coulomb potential beyond the screening length equal to the ThomasFermi radius $a_{T F}=0.885\left(\hbar^{2} / m_{e} e^{2}\right) Z^{-1 / 3}$. Using a quantum approach based on the first Born approximation, and assuming a small angle deviation and a moderate $\mathrm{Z}$ 


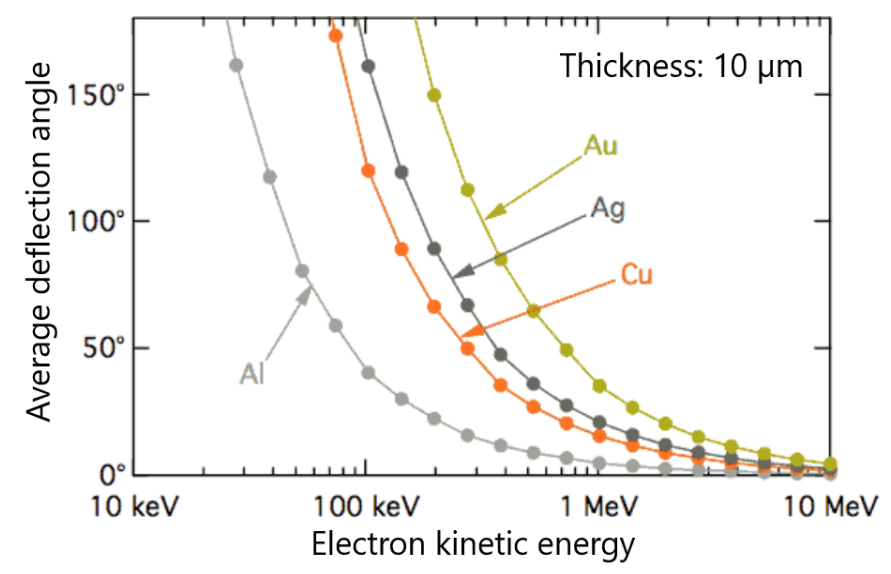

Figure 3.8: Estimation of the average electron beam scattering angle by elastic collisions after a path of $10 \mu \mathrm{m}$ for different materials considering a cold solid for a simpler use of Molière's theory and neglecting the loss of energy during propagation. Figure from [Perez, 2010].

medium, one can obtain the resulted corrected expression of the Rutherford cross section by [Nigam et al., 1959]

$$
\frac{d \sigma}{d \Omega}=4 r_{e}^{2} \frac{Z^{2}}{\gamma^{2} \beta^{4}}\left(\frac{1}{4 \sin ^{2}(\theta / 2)+\theta_{0}^{2}}\right)^{2}
$$

where we introduce the screening angle $\theta_{0}=\alpha Z^{1 / 3} /(\gamma \beta)$ with $\alpha=e^{2} /\left(4 \pi \epsilon_{0} \hbar c\right)$ the fine structure constant and the Lorentz factor $\gamma=\left(1-\beta^{2}\right)^{-1 / 2}$ has been added to take into account the relativistic effects. Finally, the screening angle removes the divergence at small angle and is generally inferior to $1^{\circ}$ and most of the collisions usually generate a smaller deflection angle. This fundamental theory, first arranged by Molière [Molière, 1947] and corrected by Nigam et al. [Nigam et al., 1959], constitutes a precise description of the angular diffusion. By averaging the number of collisions with ions, it is possible to estimate the average scattering angle using statistical treatment of multiple collisions in a material thickness as proposed by Molière in 1948 [Molière, 1948] and illustrated in figure 3.8 for different electron kinetic energy crossing different materials.

\section{Stopping Power}

Inelastic collisions happen during an electron-electron collision. During this process, a non-negligible fraction of electron kinetic energy is exchanged with the background electrons and are responsible for the slowing down of the particle. The stopping power of an electron is the electron energy loss $d E$ per unit path length $d s$ of the medium. 
It is defined as

$$
\frac{d E}{d s}=-Z E n_{i} \int_{0}^{1 / 2} \epsilon \frac{d \sigma}{d \epsilon} d \epsilon=-Z(\gamma-1) m_{e} c^{2} n_{i} \int_{0}^{1 / 2} \epsilon \frac{d \sigma}{d \epsilon} d \epsilon
$$

with $\epsilon=\Delta E / E$ the fraction of transferred energy, $n_{i}$ the density of atoms and $d \sigma / d \epsilon$ the energy transfer differential cross section. One may separate the limits of this integral between two cases: $0 \leq \epsilon \leq \epsilon_{c}$ corresponding to the contribution of collisions with bound electrons and $\epsilon_{c} \leq \epsilon \leq 1 / 2$ for the contributions of the binary collisions with free electrons, where $\epsilon_{c}$ accounts for electron average binding energy in the atomic potential and the upper limit $1 / 2$ accounts for the indiscernibility of two scattered electrons.

- In the case of a cold matter, if we consider the case $\epsilon>\epsilon_{c}$, the binding energy of the atomic electron is negligible compared to the transferred energy and so it can be consider as a collision with a free electron. The corresponding stopping power is given by Møller [Møller, 1932]

$$
\left(\frac{d E}{d s}\right)_{\text {free }}^{\text {cold }}=-E n_{i} Z \sigma_{e}\left[\ln \left(\frac{1}{4 \epsilon_{c}}\right)+1-\frac{2 \gamma-1}{\gamma^{2}} \ln 2+\frac{(\gamma-1)^{2}}{8 \gamma^{2}}\right] .
$$

with $\sigma_{e}=2 \pi r_{e}^{2} / \beta^{2}(\gamma-1)$ the electron characteristic cross section, $r_{e}=e^{2} / 4 \pi \epsilon_{0} m_{e} c^{2}$ electron classical radius. If the transferred energy is small $\epsilon<\epsilon_{c}$, one needs to take into account the binding energies of the bound electrons and all the contributions of the excited quantum states. The contribution of collisions with bound electrons in a cold solid has been determined by Bethe [Bethe, 1932] and reads

$$
\left(\frac{d E}{d s}\right)_{\text {bound }}^{\text {cold }}=-E n_{i} Z \sigma_{e}\left[\ln \left(\frac{2 \epsilon_{c} m_{e} c^{2}\left(\gamma^{2}-1\right)(\gamma-1)}{I_{0}^{2}}\right)-\beta^{2}\right],
$$

where $I_{0}=\sum_{n} f_{n} \ln \left(E_{n}\right)$ is the mean ionization potential with $E_{n}$ the energies of all the atomic levels involved and $f_{n}$ their oscillator forces. An empirical formula from [Sternheimer, 1966] reproduced the experimental value for materials with $\mathbf{Z} \geq 13$ :

$$
I_{0} \approx 9.76 Z+58.8 Z^{-0.19}
$$

The sum of the two contributions of collisions leads to the final expression of the stopping power in a cold material [Rohrlich \& Carlson, 1954; Seltzer, 1974]

$$
\begin{aligned}
\left(\frac{d E}{d s}\right)_{\text {total }}^{\text {cold }}=-E n_{i} Z \sigma_{e}[ & 2 \ln \left(\frac{m_{e} c^{2}(\gamma-1)(\gamma-1)}{2 I_{0}^{2}}\right)+1-\beta^{2} \\
& \left.-\frac{2 \gamma-1}{\gamma^{2}} \ln 2+\frac{1}{8}\left(\frac{\gamma-1}{\gamma}\right)^{2}-\delta\right]
\end{aligned}
$$




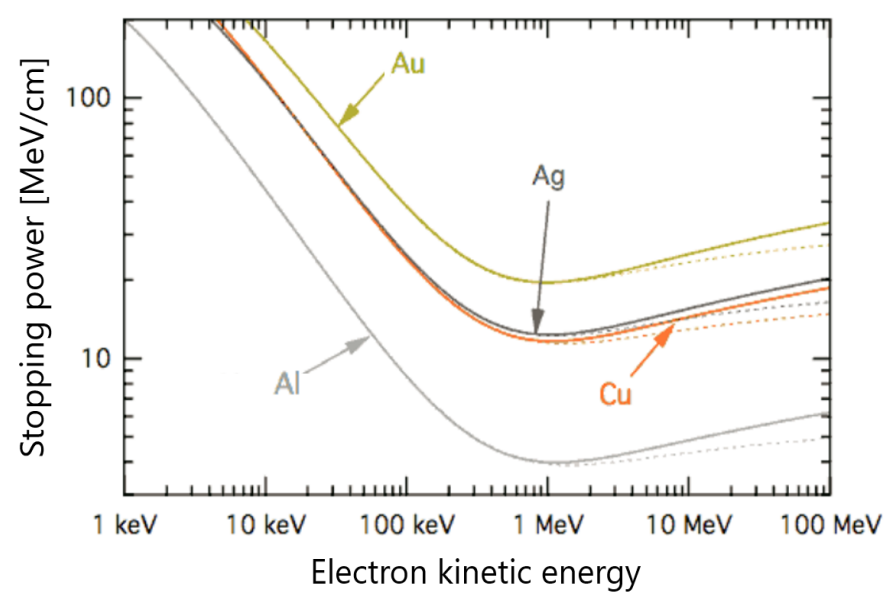

Figure 3.9: Stopping power of an electron in different cold materials according to the kinetic energy. Dotted curves represent the stopping power with the density effect correction. Figure from [Perez, 2010].

The term $\delta$ in Equation (3.45) accounts for density effect correction. For electron with high incident energy, collisions occur with impact parameters greater than the interatomic distance. The generated field of the fast electron projectile influences the surrounding atoms, modifying their electronic structure thus generating a dielectric polarization that screens the incident electron field itself. The response of a material is different from another, causing a different stopping power in each material. This effect, introduced by Fermi [Fermi, 1940] is called density effect cause it increases with the electron density. It is responsible for a decrease of the electron stopping power at energies of incidence above a few $\mathrm{MeV}$ and is represented in Figure 3.9 for different elements.

-In a ionized solid material, a part of the bounded electrons of the atom become free, affecting the stopping power. In the case of the bound electron contribution, one must modify the stopping power by replacing $Z$ by $\left(Z-Z^{*}\right)$ in order to get the real bounded electron number. The equation becomes

$$
\left(\frac{d E}{d s}\right)_{\text {bound }}^{\text {hot }}=-E n_{i}\left(Z-Z^{*}\right) \sigma_{e}\left[\ln \left(\frac{2 \epsilon_{c} m_{e} c^{2}\left(\gamma^{2}-1\right)(\gamma-1)}{I_{0}^{2}}\right)-\beta^{2}\right]
$$

with $I_{Z^{*}}$ the modified ionized potential which takes into account the ionization degree of the atom. An empirical formula from More [More, 1985], using the Thomas-Fermi model, is a good approximation of $I_{Z^{*}}$ :

$$
I_{Z^{*}}=a Z \frac{\exp \left[1.29\left(Z^{*} / Z\right)^{0.72-1.18\left(Z^{*} / Z\right)}\right]}{\sqrt{1-\left(Z^{*} / Z\right)}}
$$


with $a$ an empiric constant $\approx 10 \mathrm{eV}$.

In the case of the energy losses due to collisions with free electrons, one must replace the atomic number $Z$ by the ionization degree $Z^{*}$. It reads

$$
\left(\frac{d E}{d s}\right)_{\text {free }}^{h o t}=-E n_{i} Z^{*} \sigma_{e}\left[\ln \left(\frac{1}{4 \epsilon_{c}}\right)+1-\frac{2 \gamma-1}{\gamma^{2}} \ln 2+\frac{(\gamma-1)^{2}}{8 \gamma^{2}}\right]
$$

where $\epsilon_{c}$, has been estimated by Nardi and Zinamon [Nardi \& Zinamon, 1978]:

$$
\epsilon_{c}=\left(\frac{\lambda_{D B}}{D}\right)^{2}
$$

with $\lambda_{D B}$ the De Broglie wavelength estimated by [Solodov \& Betti, 2008] and $D$ the effective Debye length.

Another contribution to the stopping power of the electron is due to the plasma waves and was estimated by Pines and Bohm [Pines \& Bohm, 1952]:

$$
\left(\frac{d E}{d s}\right)_{p l a s}^{h o t}=-E n_{i} Z^{*} \sigma_{e} \ln \left[\left(\frac{v}{\omega_{p e} \lambda_{D} \sqrt{3 / 2}}\right)^{2}\right] .
$$

Finally, the fast electrons collisional stopping power in hot material is written as the sum of three contributions:

$$
\left(\frac{d E}{d s}\right)_{\text {total }}^{h o t}=\left(\frac{d E}{d s}\right)_{\text {free }}^{h o t}+\left(\frac{d E}{d s}\right)_{\text {bound }}^{\text {hot }}+\left(\frac{d E}{d s}\right)_{\text {plas }}^{\text {hot }} .
$$

A relativistic electron can also lose energy by emitting bremsstrahlung radiations. The Coulomb collision of an the incident electron with an ion generates a photon emission, and can be responsible for a significant fraction of the total energy losses. The photon emission is $Z^{2}$ dependent and will have a more significant effect in high $\mathrm{Z}$ material. According to Berger and Seltzer [Berger \& Seltzer, 1964], the radiative losses will become dominant for relativistic electrons with energies greater than

$$
E_{c}=\frac{800 \mathrm{MeV}}{Z+1.2}
$$

which is about $\approx 56 \mathrm{MeV}$ in aluminum and $\approx 34 \mathrm{MeV}$ in Titanium. In our case, fast electrons have relatively small energy $(E<10 \mathrm{MeV})$, the contribution due to radiative stopping power is thus negligible. The reader can refer to [Heitler \& Sauter, 1933] a deep study about this contribution. 


\subsubsection{Collective Effects}

Besides the collisional processes, other mechanisms influence the propagation of the fast electrons through matter. Indeed, when fast electron beam propagate into the dense plasma, it creates an important perturbation of the local medium neutrality and generates high density current of several $\mathrm{kA} . \mu \mathrm{m}^{-2}$. These processes are at the origin of the self induce fields, responsible for collective effects with affect the electron beam propagation. In this section, we will briefly introduce the collective electron transport into dense plasma.

\section{Neutralization of a monoenergetic fast electron beam}

A Relativistic electron beam propagating in a dense plasma will generate an electric field which will expel radially out of the beam the surrounding background electrons due to the negative charge accumulation. This charge neutralization occurs on a timescale of $\max \left[1 / \nu_{e i}, 1 / \omega_{p e}\right]$. In addition, a strong current $I_{b} \approx 10^{6} \mathrm{~A}$ due to fast electron beam propagation generates a magnetic field which tends to deviate the beam. Alfvén shows that if the field is strong enough, it can deviate the beam toward the opposite direction [Alfvén, 1939]. Lawson [Lawson, 1959] demonstrated that the current limit $I_{A}$ can be defined as the current for which the Larmor radius $R_{L}$ is equal to half the radius of the electron beam. The Alfvéen-Lawson limit reads

$$
I_{A}=\gamma_{b} \beta_{b} \frac{m_{e} c}{e} \approx 17 \gamma_{b} \beta_{b}[k A]
$$

with $\gamma_{b}=1 / \sqrt{1-\beta_{b}^{2}}$ the beam electrons Lorentz factor. This limit current is about $\approx 10^{4} \mathrm{~A}$, which is lower than the current carried by the generated fast electron beam $I_{b}$. In fact, over the same timescale than the neutralization charge, the temporal variation of the magnetic field generated by the fast electron beam induces a longitudinal electric field via the Lenzs law [Hammer \& Rostoker, 1970] that accelerates a return current $\mathbf{j}_{e}$ of background free electrons which tends to compensate the fast electron beam current density in order to get a total current $I_{t o t}=I_{b}+I_{e}$ lower than $I_{A}$. However, the magnetic neutralization is never reached completely. The conditions required to satisfy an efficient neutralization depends on the ratio between the radius of the beam $r_{L}$ and the skin depth $\lambda_{e}=c / \omega_{p e}$ [Lee \& Sudan, 1971]. When this ratio is $>1$, the current return adjusts itself to the spatial profile of the fast electron beam so that the magnetic field is considerably reduced, allowing the fast electron beam to propagate. Nonetheless, the neutralization does not last indefinitely. Indeed, due to the collisional return current, the magnetic field begins to diffuse, with a characteristic time

$$
\tau_{D}=\frac{4 \pi \mu_{0} r_{b}^{2}}{\eta}
$$

in the order of few picoseconds, with $\eta$ the resistivity of the background plasma. This 
time scale is longer than the beam duration of our experimental condition and so the neutralization is fairly efficient in our conditions.

\section{Self-generated fields}

The self generated electromagnetic field can strongly affect the fast electron propagation. In order to estimate these effects on the electron beam, we consider the model developed by Davies in 1997 [Davies et al., 1997]. The electric field can be calculated via the generalized Ohms law

$$
\mathbf{E}=\eta \mathbf{j}_{e}-\frac{1}{n_{e} e} \nabla\left(n_{e} T_{e}\right)
$$

the Maxwell-Faraday equation (CGS units)

$$
\frac{1}{c} \frac{\partial \mathbf{B}}{\partial t}=-\nabla \times \mathbf{E}
$$

and the Maxwell-Ampére equation (CGS units)

$$
\frac{\partial \mathbf{E}}{\partial t}=c \nabla \times \mathbf{B}-4 \pi\left(\mathbf{j}_{e}-\mathbf{j}_{b}\right)
$$

where Equation (3.55) is valid only for electron beam duration longer than charge neutralization (3.54). The self-generated electromagnetic field reads

$$
\mathbf{E}=-\eta \mathbf{J}_{b}+\frac{\eta c}{4 \pi} \nabla \times \mathbf{B}-\frac{1}{n_{e} e} \nabla\left(n_{e} T_{e}\right)
$$

and

$$
\frac{1}{c} \frac{\partial \mathbf{B}}{\partial t}+\frac{\partial}{\partial t} \times\left(\frac{\eta c}{4 \pi} \nabla \times \mathbf{B}\right)=\underbrace{\eta \nabla \times \mathbf{j}_{b}}_{\text {current }}+\underbrace{\nabla(\eta) \times \mathbf{j}_{b}}_{\text {resistivity }}-\underbrace{\frac{1}{n_{e} e} \nabla\left(n_{e}\right) \times \nabla\left(T_{e}\right)}_{\text {crossed gradients }} .
$$

One can see three source terms for the magnetic field generation on the right hand of Equation (3.59). The first term labeled current depends on the curl of beam current density, which generates a magnetic field responsible for the collimation of the fast electron beam [Robinson et al., 2008; Scott et al., 2012; Volpe et al., 2014]. The second term labeled resistivity describes the magnetic field generation by the resistivity gradients and tends to move the fast electron toward high resistivity zone while the resistive electric field slows down the fast electrons [Curcio \& Volpe, 2019; Davies et al., 1997]. The magnetic field generated by the third term labeled crossed gradient can affects the beam transport on a time scale of about tens of picoseconds [Nicolaï et al., 2011] and can be neglected for shorter time scales.

[Davies et al., 1997] proposed a simple model to deduce the maximum value of the 
fields $E_{\max }$ and $B_{\max }$. If one consider a Gaussian laser pulse and an almost perfect neutralization of the fast electron beam $\left(\mathbf{j}_{b} \approx \mathbf{j}_{e}\right)$, the field value reads, practical units:

$$
E_{\text {max }}\left[\mathrm{V} \cdot \mathrm{m}^{-1}\right] \approx 6 \times 10^{9}\left(\frac{\eta}{2 \times 10^{-6} \Omega \cdot \mathrm{m}}\right)\left(\frac{\eta_{L}}{0.3}\right)\left(\frac{I_{L}}{10^{17} \mathrm{~W} \cdot \mathrm{cm}^{-2}}\right)\left(\frac{1 \mu \mathrm{m}}{\lambda_{L}}\right)^{2 / 3},
$$

and

$$
B_{\max }[\mathrm{T}] \approx 230\left(\frac{\eta}{2 \times 10^{-6} \Omega . \mathrm{m}}\right)\left(\frac{2 \tau_{L}}{1 \mathrm{ps}}\right)\left(\frac{10 \mu \mathrm{m}}{r_{b}}\right)\left(\frac{\eta_{L}}{0.3}\right)\left(\frac{I_{L}}{10^{17} \mathrm{~W} \cdot \mathrm{cm}^{-2}}\right)\left(\frac{1 \mu \mathrm{m}}{\lambda_{L}}\right)^{2 / 3},
$$

where $I_{L}$ is the laser intensity, $\tau_{L}$ the pulse duration, $\eta$ the plasma resistivity, $r_{b}$ the fast electron beam radius and $\eta_{L \rightarrow e}$ conversion efficiency. According to these equations, it is possible to estimate the effect of the field on the fast electron propagation. It is shown that electromagnetic effects are dominant for laser intensities higher than $10^{18}-10^{19}$ W.cm ${ }^{-2}$ [Volpe et al., 2013].

\section{Fast electron refluxing}

When the fast electron beam escapes from the rear side of the target, the charge separation induces an intense electric field which tends to bring back the electrons inside the target. Different models can be find in the literature to estimate the circulation rate of the electrons [Fill, 2005; Myatt et al., 2007]. Reducing the target allows to get a higher electron beam density on the rear side of the target. Many studies have been done concerning the contribution of the refluxing in the proton acceleration mechanism. Mackinnon et al. [Mackinnon et al., 2002] observed that for thin target, the electron refluxing phenomenon is also responsible for an enhancement of the maximum accelerated proton energy. Indeed, it the target is thin enough, the electron leaving and entering the target will superposed, increasing thus the effective electron density, responsible a higher charge electric field separation.

\subsection{Target Normal Sheath Acceleration}

The Target Normal Sheath Acceleration (TNSA) is the dominant mechanism for energetic ion generation achievable with current laser technology. It has been extensively studied in experiments and simulations all over the world [Mora, 2003; Perego et al., 2012; Schreiber et al., 2006] and was introduced by [Snavely et al., 2000; Wilks et al., 2001]. In the TNSA scheme, an intense laser pulse is impinging on a front side of a solid target of the order of micrometers. The electromagnetic energy is then transferred to the electron of the target by different heating mechanisms (mentioned in the previous section 3.2.3). This electronic population of hot electron produced during the interaction then propagates through the target until reaching the rear side. We saw before that only the part of the highest energies of this population will be able 
to escape from the target while most of the electrons will be recalled into the target because of the electrostatic fields generated by the charge separation at the interface of the target with the vacuum. The electrons can thus move away from the target with a characteristic length equal to the Debye length and establish, at its boundaries, a huge electro-static fields of the order of $\mathrm{TV} / \mathrm{m}$. This sheath field is oriented normally to the target, and therefore the ions are accelerated in the perpendicular direction regardless of the incidence angle of the incoming laser pulse; hence the name Target Normal Sheath Acceleration. In this framework, protons are preferentially accelerated due to their high charge-to-mass ratio and are coming from the superficial impurity layer of the target. However, heavier ions, again from the impurity layer or from the bulk, can reach relevant energies [Roth et al., 2013]. This section will concentrate first on laser driven proton by TNSA. The Slowing Down Theory of Non-Relativistic Protons and multiple scattering model used in the framework of this thesis will be then introduce.

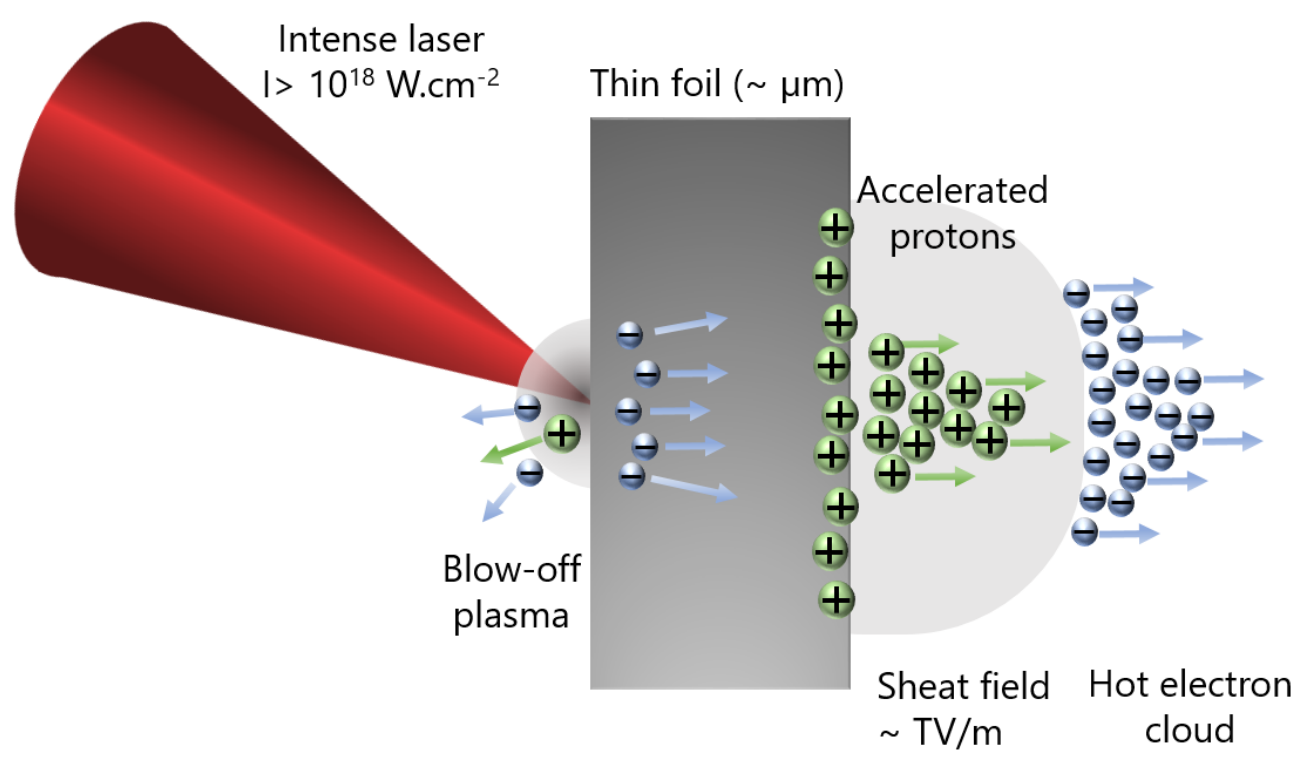

Figure 3.10: TNSA mechanism. A high-intensity laser pulse is focused onto the front side of a thin target foil, generating a blow-off plasma and thus accelerating a copious amounts of hot electrons. The electrons propagate through the foil and set up a strong charge separation field at the rear side of the target. Protons and other atoms, present as hydro-contaminants at the back surface of the foil, are subsequently pulled out and accelerated to high energies.

\subsubsection{Mechanism}

Many scaling laws have been developed to estimate the accelerated ion peak energy $E_{\max }$. These descriptions give a simplified picture of the acceleration process 
in different descriptive approaches such as the well known isothermal plasma expansion model [Mora, 2003], the cylindrical electro-static description of [Schreiber et al., 2006] or the quasi-static model proposed by [Lontano \& Passoni, 2006] (the interested reader may refer to [Perego, 2013] for further studies). The simple analytical model of [Schreiber et al., 2006], which gives very good theoretical approach to this mechanism, will be describe below.

This model assumes that the hot electron expansion in vacuum creates a cylindrical quasi-static cloud in the vacuum on the back side of the target, creating a circular positive surface charge on its rear face. The generated electrostatic potential is evaluated on the symmetry axis, along which the most energetic ions are accelerated. We consider that $N_{e}$ electrons are accelerated by a relativistic laser pulse of pulse duration $\tau_{L}$ from the target front side to an mean kinetic electron energy $E_{e}$. The hot electron population number accelerated by the laser with energy $E_{L}$ can be estimated by

$$
N_{e} E_{e}=\eta_{L} E_{L}
$$

with $E_{e}=k_{B} T_{e}$ estimated by the ponderomotive scaling equation (3.37), $\eta_{L}$ is the conversion efficiency of the laser energy into hot electrons generally considered between 10 and $50 \%$. The electron bunch of length $\tau_{L} c$ propagates (electrons are assumed to propagate with the speed of light $c$ ) through the foil with a thickness $d$ and are spread at the rear side of the foil over a circular area of radius

$$
R=r_{L}+d \cdot \tan (\theta)
$$

where $r_{L}$ corresponds to the radius of the laser spot and $\theta$ the half-angle of the propagation cone. As a consequence, the presence of a cylindrical negatively charged cloud induces a positive surface charge density which yields a returning force $\propto Q e /\left(\pi R^{2}\right)$ ( $Q$ is the hot electron quantity) at the rear side of the target, located at $z=0$ (where $z$ is the electron propagation axis). The generated electro-static potential can be evaluated via Poisson equation in a cylindrically symmetric geometry where $\mathrm{r}$ denotes the radial coordinate

$$
\Phi(r, z)=\frac{1}{4 \pi \epsilon_{0}} \cdot \frac{Q e}{\pi R^{2}} \cdot \int_{0}^{R} \int_{0}^{2 \pi} \frac{r^{\prime} d r^{\prime} d \phi^{\prime}}{\sqrt{z^{2}+r^{2}+r^{\prime 2}+2 r r^{\prime} \cos (\phi)}} .
$$

At the $\mathrm{z}$ axis $(\mathrm{r}=0)$, region that is responsible for the most energetic ions, this integral can be evaluate and gives

$$
-e \Phi(r=0, \xi)=E_{\infty} s(\xi), \quad \text { with } \quad E_{\infty}=\frac{Q e^{2}}{2 \pi \epsilon_{0} R^{2}}
$$

where $\xi=z / R$ stands for the normalized propagation direction normal to the foil and $s(\xi)=1+\xi-\sqrt{1+\xi^{2}}$. Only a few electrons with energies exceeding $E_{\infty}$ can escape the rear surface of the potential barrier whereas the low energetic electrons of energy $E_{e}$ are forced to turn around at a distance $\xi_{u}=z_{u} / R$ with $\xi_{u}=E_{e} / E_{\infty}$ 
assuming $\xi_{u} \ll 1$. Using the definition of $E_{\infty}$ and the electron density expression $n_{Q, 0}=Q /\left(\pi R^{2} z_{u}\right)$ directly at the surface one derives

$$
z_{u}=\frac{E_{e}}{E_{\infty}} \cdot R=\left(\frac{2 \epsilon_{0} k_{B} T_{e}}{n_{Q, 0} e^{2}}\right)^{\frac{1}{2}},
$$

where $n_{Q, 0}=2 n_{e, 0}$ is composed from electrons with density $n_{e 0}$ streaming out of and back into the foil with

$$
n_{e 0}=\frac{N_{e}}{c \tau_{L} \pi B^{2}} .
$$

Thus by inserting (3.67) inside (3.66), we can deduce the earlier definition of the Debye length $\lambda_{D}$ from (2.31). Then the surface electric field at the vacuum - solid interface $(\mathrm{z}=0)$ reads

$$
F(z=0)=\frac{k_{B} T_{e}}{e \lambda_{D}}
$$

and is consistent with the one resulting from the established plasma expansion model [Mora, 2003]. In the equilibrium situation $Q=2 N_{e} z_{u} / c \tau_{L}$ electrons are permanently outside the foil, which, in turn, generate $Q$ positive charges in order to maintain the neutrality. It allows for the rewriting of the potential barrier $E_{\infty}$ as

$$
E_{\infty}=2 m_{e} c^{2} \sqrt{\frac{\eta_{L} P_{L}}{P_{e}}}
$$

where $P_{e}=m_{e} c^{3} / r_{e}=8.7 G W$ is the relativistic power unit. The proton energy is then calculated from the potential caused by the induced surface charge at the actual position of the proton $\xi$ and reads

$$
E_{p}(\xi)=-q e \Phi(r=0, \xi)=q E_{\infty}\left(1+\xi-\sqrt{1+\xi^{2}}\right),
$$

with $q e$ the proton charge and $q=1$ for proton.

More in detail, the maximum ion energy $E_{\mathrm{p}, \max }$ can be evaluated analytically by integrating the equation of motion up to the duration of the laser pulse via an implicit function and gives

$$
E_{\mathrm{p}, \max }=E_{\infty} \tanh ^{2}\left(\frac{\tau_{L}}{2 \tau_{0}}\right)
$$

with $\tau_{0}=R / v_{\infty}=R /\left(2 E_{\infty} / m_{p}\right)^{1 / 2}$ the non-relativistic characteristic time which give the time for which a proton remains in the vicinity of the accelerating surface. This theoretical model introduces the appearance of an optimal pulse duration at fixed laser energy, represented in Figure 3.11. We note that the estimation of $\eta_{L}$ is approximated by an empirical scaling[Mora, 2003]:

$$
\eta_{L}=\left(1.2 \times 10^{-15}\right) \times\left(I_{L}\right)^{3 / 4}
$$

giving a maximum value up to $\eta_{L}=0.5$ reached for a laser intensity of $3.1 \times 10^{19} \mathrm{~W} . \mathrm{cm}^{-2}$. 


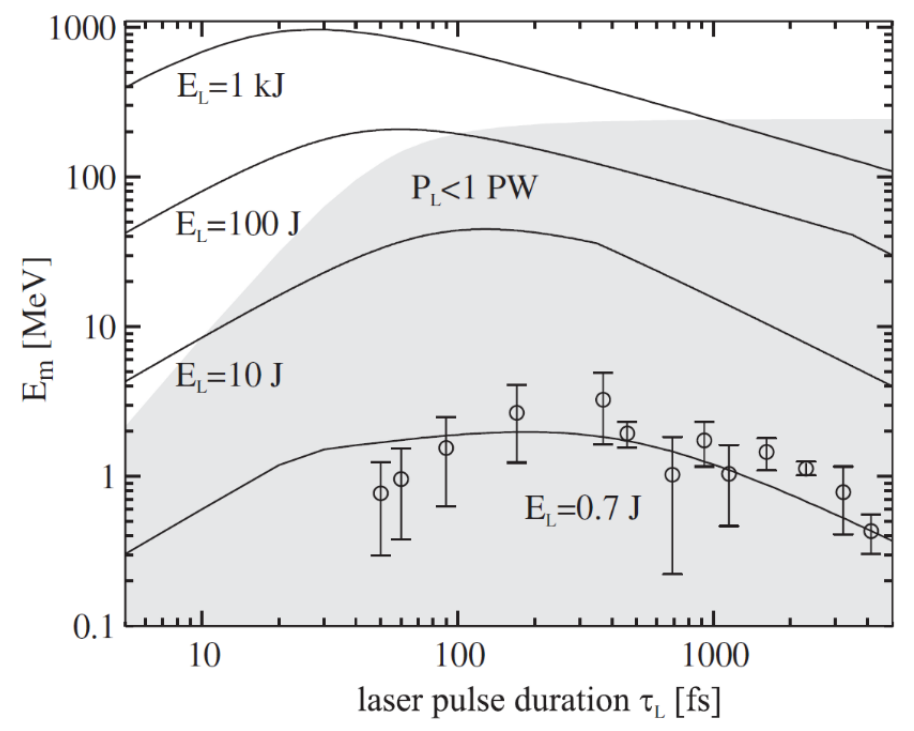

Figure 3.11: Maximum proton energy $E_{\mathrm{p}, \max }$ as a function of the laser pulse duration $\tau_{L}$ for four constant laser energies $E_{L}$. Theoretical results (solid lines) are plotted following the equation (3.71) with the experimental data (circles) as support for the scaling model. Parameters of the fit are $r_{L}=4 \mu \mathrm{m}$, electron half angle divergence $\phi$ $=10^{\circ}$ and target thickness $=10 \mu \mathrm{m}$. Image taken from [Schreiber et al., 2006].

\subsubsection{Main experimental achievements}

A large number of experimental campaigns have been carrying out during the last decades, clarifying several features of the TNSA mechanism. The team of [Hatchett et al., 2000] and [Snavely et al., 2000] highlighted a key property of TNSA in the 2000s. Using a wedge-shaped target (see Figure 3.12), they observed two separated proton beams in the direction normal to the two rear surfaces of the wedge, thus confirming that the proton emission is mainly originating from the rear surface of the target, along its normal direction.

In 2001, [Mackinnon et al., 2001] studied the influence of the foil rear surface density profile on the ion emission by using targets with and without preformed plasmas. The maximum proton energy efficiency was found to be strongly enhanced by short density scale length, in agreement with the TNSA mechanism.

The team of [Hegelich et al., 2002] succeeded to accelerate high-energy C, Ca and F ions from prepared target of $\mathrm{Al}$ and $\mathrm{W}$ where the $\mathrm{CH}$ contaminant at the rear side of the target were removed by resistive heating technique. The target foils were then coated by thin layer of $\mathrm{C}$ and $\mathrm{CaF}_{2}$ on the rear side. Figure 3.13 shows the different ion species accelerated which proves the existence of an effective rear surface acceleration mechanism. In addition, it demonstrates that the surface cleaning technique can reduce the rear side screening effects from proton, permitting an acceleration of heavier ion species. A direct evidence of the generation of an initial intense sheath 

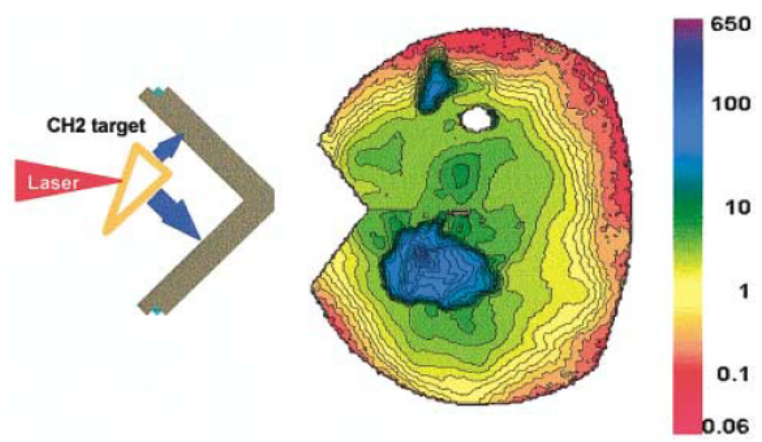

Figure 3.12: Scheme of wedge target irradiation. The proton emission was detected on the rear side of the wedge target by radiochromic films. Two separated spots are produced on the film and a larger particle flux can be observed on the major side of the target. From [Snavely et al., 2000].

field at the rear surface has been explore by [Romagnani et al., 2005] by using a proton beam as a transverse probe of the sheath field.

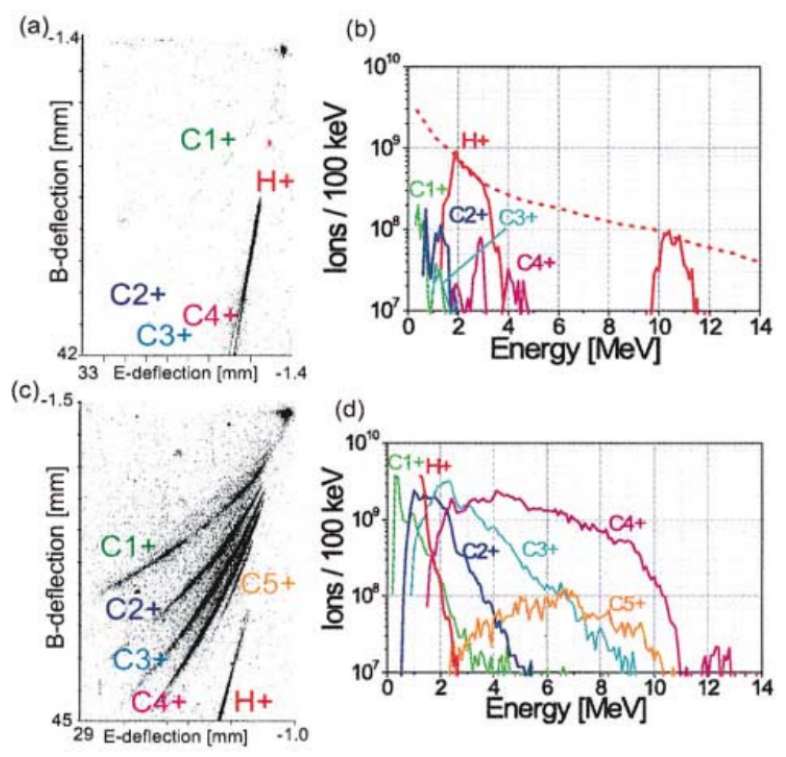

Figure 3.13: Different ion signal patterns and corresponding spectra in the presence of hydrocarbon contaminants on the rear surface ((a) and (b)) and after its elimination ((c) and (d)) by resistive heating. From [Hegelich et al., 2002].

Evidence of high laminarity and collimation of the beam have been demonstrated in the experiment carried out by [Cowan et al., 2004]. Thin metallic grid of shallow grooves were placed at the rear surface of a target to induce a phase space modulations on the proton beam, allowing diagnosing the proton beam. As shown in 
Figure 3.14, the ion beam has transferred the grid imprint on the radiochromic film as a modulation of the particle flux. The collimation of the beam was found to be inverse proportional to the proton energy with a divergence $<10^{\circ}$ for proton $>10$ $\mathrm{MeV}$ and a transverse emittance $(<0.004 \mathrm{~mm} \mathrm{mrad})$ around 100 times lower than in a conventional $\mathrm{RF}$ accelerator.
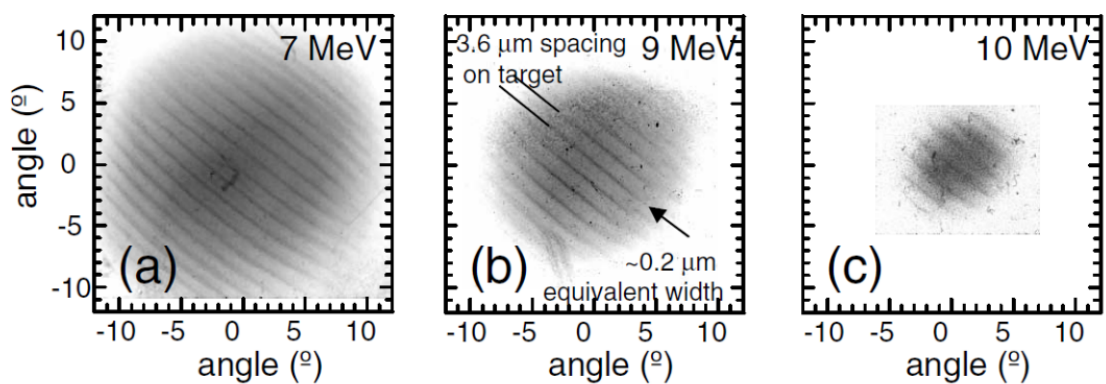

Figure 3.14: Detection of rear surface shallow-groove imprint in multiple layers of radiochromic film (RCF) corresponding to specific interval of proton energy 7, 9 and $10 \mathrm{MeV}$. From [Cowan et al., 2004].

\subsubsection{Beam optimization}

Many experiments have been performed in order to optimize the quality of the accelerated proton beam by adjusting the laser parameters (prepulse, intensity, pulse duration...) and the structure of the targets (thickness, composition, surface area...). [Mackinnon et al., 2002] demonstrated the enhancement of the proton maximum energy varying the target thickness. The experimental results showed a proton cut-off at $24 \mathrm{MeV}$ with a $3 \mu \mathrm{m}$ target while this energy drops to $6.5 \mathrm{MeV}$ when a $100 \mu \mathrm{m}$ target was used. This can be explained by the fact that thinner targets facilitate the recirculation of electrons, implying a higher mean density of the hot electron, and thus, stronger electric field, at the target rear surface. This clearly confirms that proton energy is related to the sheath electric field at the rear target. The team of [Spencer et al., 2003] studied the influence of the laser prepulse due to amplified spontaneous emission (ASE) on proton emission for different material (Al, Mylar) and target thickness (0.8 to $50 \mu \mathrm{m}$ and 13 to $90 \mu \mathrm{m}$ ). It was demonstrated that in the presence of a low contrast prepulse, the proton cut-off energy increases up to an optimum thickness, and then falls off for thinner targets. The decrease in proton peak energy after the optimum thickness can be the consequence of the fast electrons losing energy through collisions when traveling through the target. For thinner targets than the optimum one, a preplasma formation at the rear side can perturb the proton emission. [Kaluza et al., 2004] has been investigated in detail the influence of the prepulse duration on the acceleration of protons in thin-foil target. In this experiment, $\mathrm{Al}$ foils of different thicknesses $(0.85$ to $86 \mu \mathrm{m})$ were considered in the presence of an ASE prepulse (with a contrast ratio $>2 \times 10^{7}$ ) with a duration that 


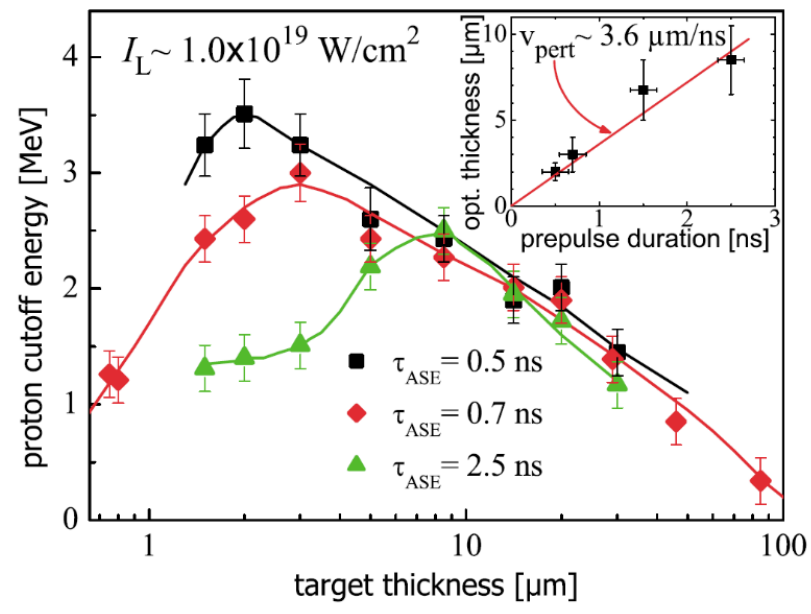

Figure 3.15: Proton cut-off energies for different ASE prepulse durations and target thicknesses. From [Kaluza et al., 2004].

could be controlled from $0.5,0.7$ and 2.5 ns (see Figure 3.15). The corresponding results shows that proton cut-off energy depends strongly on the ASE prepulse with a linear dependence of the target optimal thickness with the ASE duration. A maximum proton number up to $10^{9}$ protons $/ \mathrm{MeV} / \mathrm{mrad}$ was obtained for proton energy $>0.5 \mathrm{MeV}$ (see Figure 3.16). Laser technology with ultra-high laser contrast

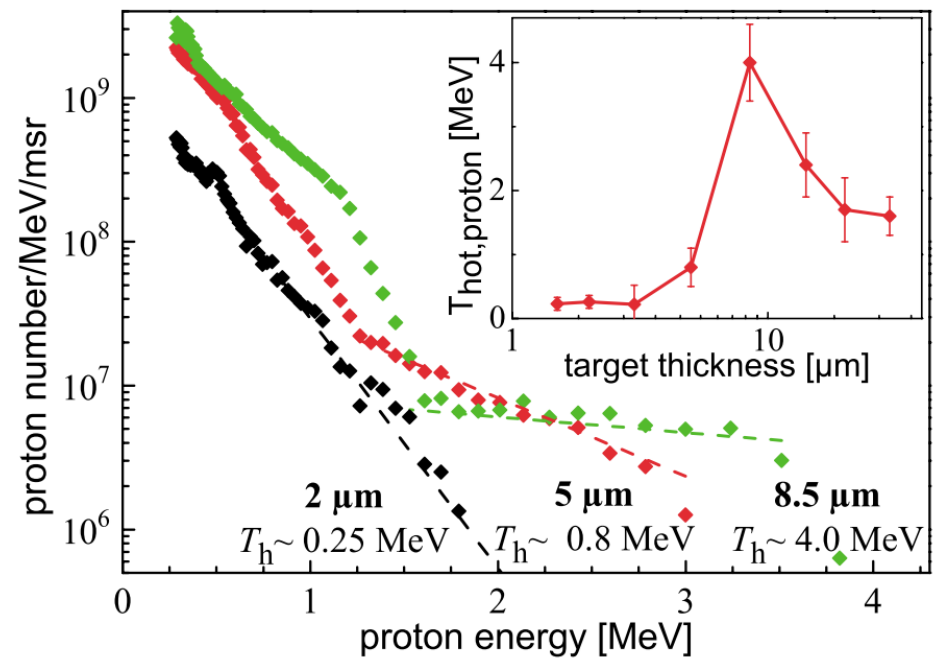

Figure 3.16: Proton energy spectrum obtained by irradiating foils of different target thicknesses with intensity $I_{L}=1.3 \times 10^{19} \mathrm{~W} . \mathrm{cm}^{-2}$. From [Kaluza et al., 2004].

can significantly improve the proton effective acceleration process. Indeed, without a pre-formation and pre-heating of the target rear surface plasma due to prepulse, 
the refluxing and thus the hot electron concentration will be consequently improved. [Ceccotti et al., 2007] study the proton cut-off energy as a function of target thickness for a high-contrast $\left(\mathrm{HC}=10^{-10}: 1\right)$ and a low-contrast $\left(\mathrm{LC}=10^{-6}: 1\right)$ laser with an ion detection in forward (FWD) and backward direction (BWD) (see Figure 3.17). It has been observed that the LC results show the existence of an optimal target thickness similar to [Kaluza et al., 2004]. A symmetric behavior concerning the BWD and FWD conditions was observed with an increase of proton cut-off energy for thinner target confirming the universality of the TNSA process, which may occur at the both target sides. Other strategies have been developed to enhance the

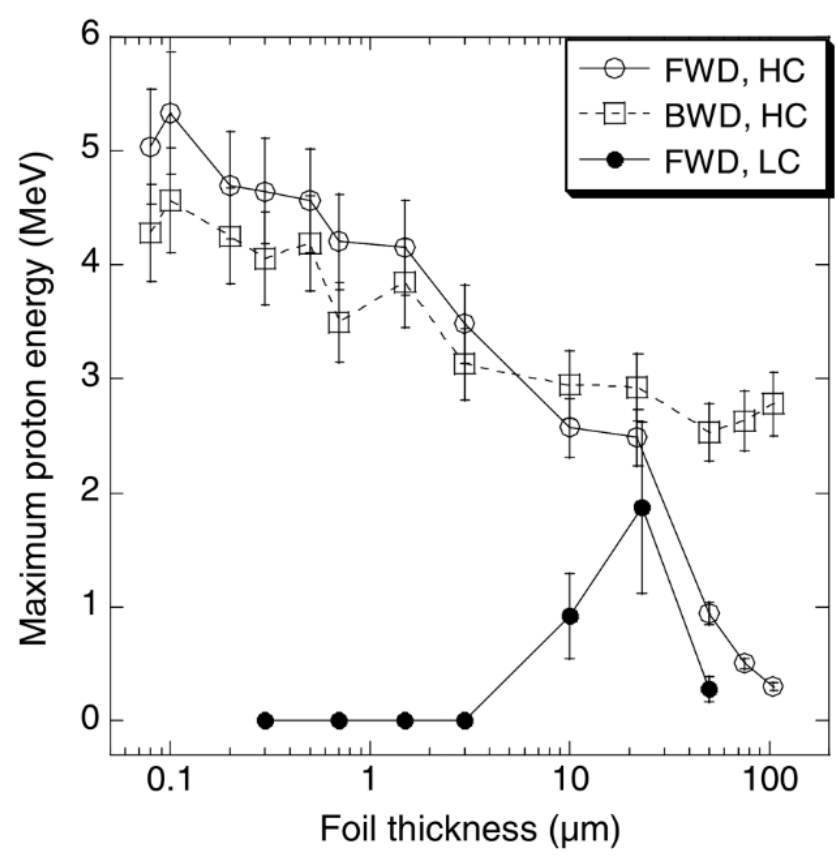

Figure 3.17: Proton cut-off energy as a function of target thickness for high-contrast (HC) and low-contrast (LC) in backward (BWD) and Forward (FWD) ion detection conditions. From [Ceccotti et al., 2007].

accelerating field formation such as the mass-limited targets technique with specific geometry and a reduction of target transversal dimension or conical-shaped targets. Numerical investigations [Psikal et al., 2008] show that surface reduction may provide further confinement of the hot electron population at rear side of the target and thus an increment in the electric field. These numerical predictions have been confirmed by experimental studies with plastic spherical targets [Henig et al., 2009] or planar $\mathrm{Au}$ targets [Buffechoux et al., 2010] (see figure 3.18). The role of the laser pulse duration as well as its intensity and energy has been proved to be a key factor in TNSA acceleration mechanism. Several investigations have been done to determine the cut-off energy and parametric studies of its dependence on laser pulse intensity, duration, power, irradiance, energy, fluence [Flacco et al., 2010; Flippo et al., 2008; 

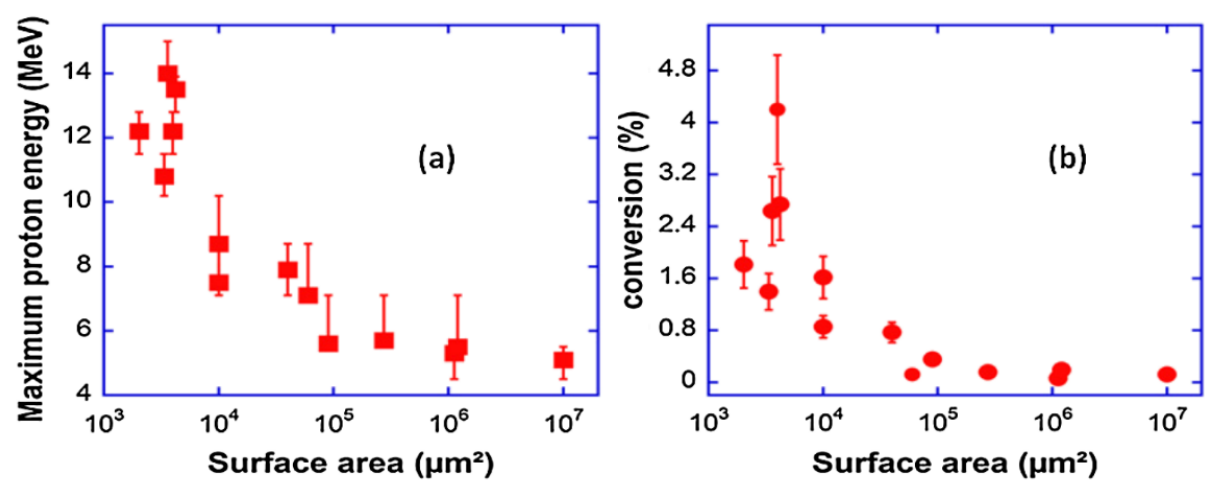

Figure 3.18: Maximum proton energy (a) and laser-to-proton energy conversion efficiencies (b) for a $2 \mu \mathrm{m}$ Au target of various surface areas irradiated by a laser pulse with intensity $I_{L}=2 \times 10^{19} \mathrm{~W} . \mathrm{cm}^{-2}$ and a duration of 400 fs. From [Buffechoux et al., 2010].

Fuchs et al., 2006; Robson et al., 2007; Zeil et al., 2010] and a general behavior of maximum ion energy, as a function of laser intensity for a given pulse duration has been observed. Figure 3.19 summarize the state of the art in terms of maximum proton energy. Different experimental parametric studies have been compared to model prediction from theoretical investigations and/or PIC simulations.

The highest TNSA energies reported so far (not updated in Figure 3.19) are of the order $67.5 \mathrm{MeV}$ [Gaillard et al., 2011] $\left(E_{L} \sim 80 \mathrm{~J}, \tau_{L} \sim 670 \pm 130 \mathrm{fs}\right)$ and $85 \mathrm{MeV}$ [Wagner et al., 2016] ( $\left.E_{L} \sim 160-200 \mathrm{~J}, \tau_{L} \sim 500-800 \mathrm{fs}\right)$ using flat-top cone and submicrometer thick plastic targets, respectively. However, during these last years, a strong development of facilities having ultra-short and ultra-intense Ti:Sa laser systems $\left(\tau_{L}<50 \mathrm{fs}, E_{L} \sim\right.$ few joules) working at high repetition rate opened the access to several applications of laser-accelerated ion beams. Results in facilities such as HRDZ (Draco) have reported maximum proton energies $<20 \mathrm{MeV}$ [Zeil et al., 2010] and $33 \mathrm{MeV}$ [Green et al., 2014] from RAL (Gemini) facility. The last performances have been done by the team of [Ogura et al., 2012] (JAEA, laser J-KAREN) and [Kim et al., 2013] (APRI, laser Pulser) reporting a record in maximum proton energies of $40 \mathrm{MeV}$ and $45 \mathrm{MeV}$, respectively.

\subsection{Proton interaction with matter}

Protons passing through matter interact primarily through coulomb forces with the atoms or molecules constituting the medium. It ensues losses of energy and deflection of the proton from its original straight-line trajectory. It is important to understand and identify the different mechanisms in order to properly built and anticipate the proton beam trajectory through our detector and also for better un- 


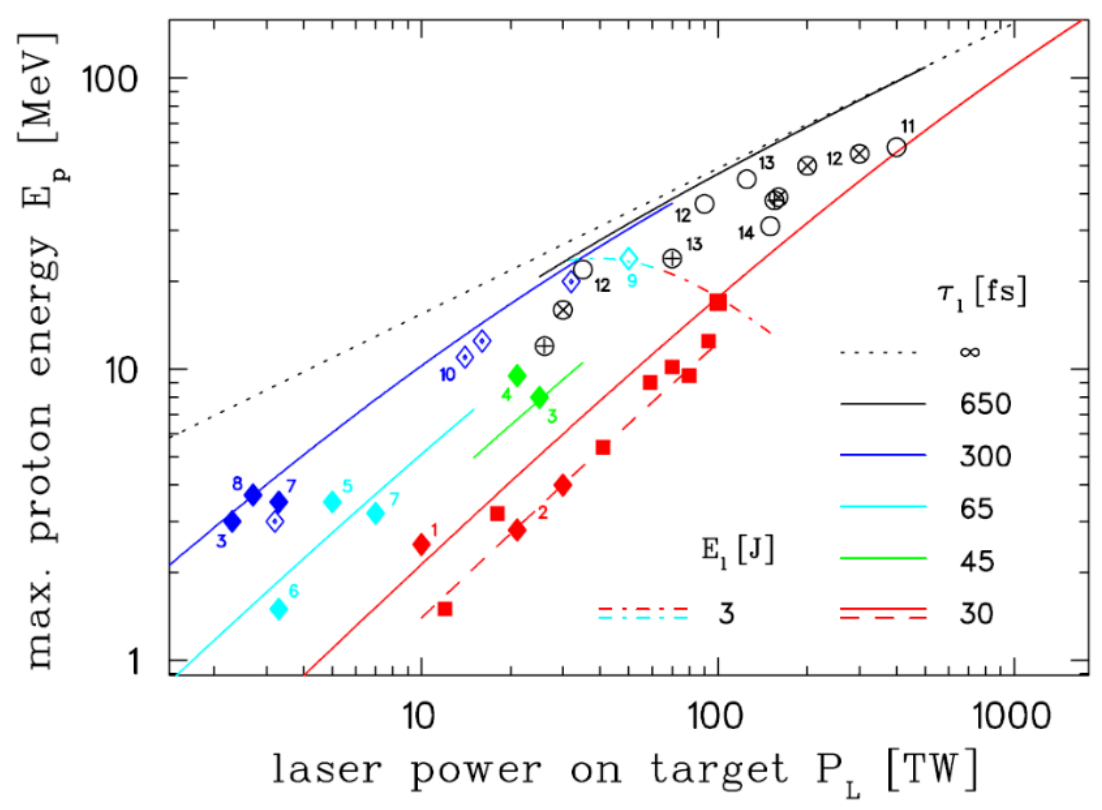

Figure 3.19: Maximum proton energy dependence on laser power, for several laser systems. Colors indicate different pulse duration regimes. Square symbols represent experiments done at the DRACO laser (HDZR, Dresden). Other points are data from other laboratories. Fitting lines correspond to the analytical model proposed by [Schreiber et al., 2006] with different pulse duration regimes (refer to colors). Further details about the figure can be found in [Zeil et al., 2010]. Figure taken from [Zeil et al., 2010].

derstanding of the dose distribution produced by protons. In this section, we will review the stopping power theory which is at the origin of the proton energy loss in a material. We will then focus on the multiple Coulomb scattering theory, necessary to estimate the amount that a proton is deflected by scattering.

\subsubsection{Proton Stopping Power theory}

The Stopping power and energy dissipation of charged particles through matter has been a subject of interest since the very early days of modern atomic physics due to the wide interest of such information for the study of nuclear physic, shielding, detector design, radiotherapy (biological effect, radiation damage)... One of the pioneers in the study of this physical mechanism was [Bohr, 1913] who defined a theoretical model based on a classical approach, later followed by [Bethe, 1930, 1933] with the development of quantum mechanics approach. Although the subject has been reviewed over the years by several authors, a perfect description of proton stopping power in cold matter has not been achieved yet, especially around the maximum stopping power. However, numerous experimental studies have been carried out and 
scaled to existing theoretical models, allowing a good understanding of the rangeenergy relations of the charged particle penetration in matter. Most of the work is either based on Bethe expression with various corrections or on empirical formulas extracted from the experimental results. A detailed overview can be find in [Berger et al., 1993a].

\section{Definition}

The stopping power $\mathrm{S}(E)$ of a material is defined as the energy loss $d E$ per unit length $d x$ in which charged particles (also called projectile) suffer when crossing a given material, due to Coulomb interactions. It reads

$$
S(E)=-\frac{d E}{d x}
$$

and is generally measured in $[\mathrm{MeV} / \mathrm{cm}]$. The stopping power depends on the type and energy of the particle and on the properties of the material it passes through. The energy loss $\Delta E$ is then given by the integral along the beam trajectory

$$
\Delta E=-\int \frac{d E}{d x} d x
$$

For a proton crossing a material at ambient conditions, the total stopping power can be divided into two parts: a principal contribution from the electronic stopping power $-(d E / d x)_{e l e}$ due to inelastic collision with bound atomic electrons and a smaller contribution from the nuclear stopping power $-(d E / d x)_{n u c}$ due to elastic collision with atomic nuclei, which becomes important at very low energies but won't be detailed in this thesis (the interested reader can refer to [Berger et al., 1993a] for further information).

\section{Electronic stopping power formula}

According to Bethe theory [Bethe, 1933; Bethe \& Ashkin, 1953], the contribution of collisions with atomic electrons can be written [Berger et al., 1993a]

$$
\left(\frac{d E}{d s}\right)_{\text {ele }}=-4 \pi \frac{n_{e} e^{4}}{m_{e} v^{2}} L
$$

Here, $e$ is the elementary charge, $m_{e}$ is the electron mass, $n_{e}$ is the atomic electron density and $v$ is the proton velocity. The quantity L takes into account the fine details of the energy loss process and is written as a sum of three term:

$$
L=L_{0}+L_{1}+L_{2}
$$

The first term is the main contribution and reads

$$
L_{0}=\frac{1}{2} \ln \left(\frac{2 m_{e} c^{2} \beta^{2} W_{\max }}{I\left(1-\beta^{2}\right)}\right)-\beta^{2}-\frac{C}{Z}-\frac{\delta}{2},
$$


with

$$
W_{\max }=\frac{2 m_{e} c^{2} \beta^{2}}{1-\beta^{2}}\left[1+\frac{2 m_{e}}{m_{p}}\left(1-\beta^{2}\right)^{-\frac{1}{2}}+\left(\frac{m_{e}}{m_{p}}\right)^{2}\right]^{-1}
$$

the largest possible energy loss by the proton in a single collision with a free electron. $m_{p}$ is the proton mass, $\beta=v / c$ and $c$ the velocity of light in vacuum, $I$ is the mean excitation energy of the material where the proton is propagating through, $C / Z$ is the shell correction ( $\mathrm{Z}$ being the atomic number of the target atom), $\delta / 2$ is the density-effect correction. As the proton kinetic energy decreases while propagating in the material, the contribution to the stopping power from interactions with bound atomic electrons in the $\mathrm{K}, \mathrm{L}, \mathrm{M}, \ldots$-shells decreases and a correction term $C / Z$ must be taken into account; see [Walske, 1952] for K-shell corrections, [Khandelwal, 1968] for L-shell corrections and [Bichsel, 1991, 1983, 1992] for M-shell corrections and above. For relativistic proton kinetic energies, the stopping power is reduced due to the resulting electrical polarization of the medium Fermi [1940]; Sternheimer [1952]; Sternheimer et al. [1982] and the density effect correction must be considered. However, considering only non-relativistic protons, this term can be neglected in all the following. The last two terms of equation (3.76) are important only for low projectile velocities. The term correction $L_{1}$ is the Barkas correction accounting for discrepancies between negatively and positively charged projectiles [Barkas et al., 1956, 1963]. Finally, the second stopping number correction $L_{2}$ provides the valid electronic stopping power expression when the proton velocity is large compared to the velocity of bound atomic electrons [Bloch, 1933; Bohr, 1948]. However, Bethe theory (3.75) breaks down when the proton velocity is much lower than the orbital electron velocities. [Varelas \& Biersack, 1970] compiled many experimental and theoretical results [Andersen \& Ziegler, 1977; Lindhard \& Winther, 1964; Newton et al., 1975] and provide a fitting formula for the electronic stopping power contribution in this low velocity regime.

\subsubsection{Coulomb Multiple scattering}

The theory of multiple Coulomb scattering of charged particles by material was developed several years ago in many papers [Bethe, 1953; Goudsmit \& Saunderson, 1940; Molière, 1948; Scott, 1963]. However, [Rossi \& Greisen, 1941] developed a simple method usually used by experimentalists, which gives rough approximation to the scattering but is enough as first approximation, and will be used in the framework of this thesis. The formula has been improved by [Highland, 1975], followed then by [Lynch \& Dahl, 1991] and gives an estimation of the lateral spreading of the beam as it passes through a sample, related to the root-mean-square angle $\left(\theta_{R M S}\right)$ of deflection arising from multiple small-angle scattering when the energy loss can be neglected. The deflection $\theta_{R M S}$ of a proton with kinetic energy $E_{k}$ traversing a material with 
density $\rho$ along the z-axis reads

$$
\theta_{R M S}[\mathrm{rad}]=\frac{E_{s}[\mathrm{MeV}] z}{2 E_{k}[\mathrm{MeV}]} \sqrt{\frac{A_{s}\left[\mathrm{~g} / \mathrm{cm}^{2}\right]}{X_{0}\left[\mathrm{~g} / \mathrm{cm}^{2}\right]}}\left[1+\epsilon \log _{10}\left(\frac{A_{s}\left[\mathrm{~g} / \mathrm{cm}^{2}\right]}{X_{0}\left[\mathrm{~g} / \mathrm{cm}^{2}\right]}\right)\right]
$$

with $E_{s}=13.4 \mathrm{MeV}$ [Lynch \& Dahl, 1991], z=1 is the proton charge, $\epsilon=0.088$ the correction parameter, $A_{s}$ is the areal density of the material of thickness $\Delta z$ such as

$$
A_{s}=\int_{z}^{z+\Delta z} \rho(z) d z
$$

and $X_{0}$ is the radiation length

$$
\frac{1}{X_{0}}=4 \alpha N_{A}\left(\frac{Z^{2}}{A}\right) r_{e}^{2} \ln \left(\frac{181}{Z^{-1 / 3}}\right) \approx 1.4 \times 10^{-3}\left(\frac{Z^{2}}{A}\right) \ln \left(\frac{181}{Z^{1 / 3}}\right)
$$

with $\alpha$ the fine-structure constant, $N_{A}$ the Avogadro's number, $r_{e}$ the electron classical radius, $\mathrm{Z}$ and $\mathrm{A}$ the atomic and mass number of the material, respectively. However, the radiation length was taken from [Tsai, 1974] which gives a more accurate number.

\subsection{Proton beam Diagnostics}

Laser-driven proton source applications generally require well-diagnosed and controlled beams with high stability. Thus, a spatial and energy characterization of the proton beams plays an important role for the potential use of such sources. The laser-driven proton source characterization requires diagnostics capable of detecting a large number of particles included inside a very broad spectrum (tens of $\mathrm{MeV}$ ) over a very short duration. Where possible, the method to diagnose the proton source should be non-destructive and operational at high repetition rate. Contrary to conventional ion accelerators, laser-driven proton detection must be robust enough to stand hostile environment of laser-plasma interaction near the source and resist to eventual electromagnetic pulses that are generated during high-intensity laser-plasma interaction experiments [Marco et al., 2016]. This report is a brief overview of established diagnostic techniques for laser-driven proton beam used in the framework of this thesis.

\subsubsection{Time of Flight techniques}

The Time-of-Flight (ToF) technique is an effective diagnostic tool so far used as on-line method for low-energy laser accelerated proton and ion beam spectroscopy [Woryna et al., 1996]. Such detector are typically placed at a finite distance from the target to determine the charged particle velocity by measuring the time required for the particle to reach the detection area. It also provides direct measurement for a 
detailed study of the ion spectrum and cut-off energy, flux [Margarone et al., 2011] as well as shot-to-shot reproducibility. The TOF signal generated by the readout system it usually composed by a narrow hard X-ray peak followed by the hot plasma electrons and a broad ion/proton peak incoming with a specific TOF and consequently kinetic energy, allowing easy discrimination of the signal originated from ions/protons (see Figure ??). Distance detector-target is a crucial point for a good detection. Indeed, the detector must be far enough for each particle species to be drifted apart and thus to distinguish the contribution of particle and resolve a sufficient small energy step within the ion/proton bunch. The temporal resolution of the measure will depend on the energy increment required between two successive proton energy measurements. However, this temporal resolution is limited by the natural resolution of the overall system composed by the TOF detector and digital system acquisition and can be expressed for non relativistic proton energy by the following condition:

$$
\Delta t_{\text {resolution }}=\sqrt{\frac{m_{p}}{2}} \times \Delta x_{T O F} \times\left(\frac{1}{\sqrt{E_{1}}}-\frac{1}{\sqrt{E_{2}}}\right) \geq \Delta t_{\text {system }}
$$

where $\Delta x_{T O F}$ is the distance detector-target, $E_{2}$ and $E_{1}$ the proton kinetic energy detectable after an energy increment $\Delta E=E_{2}-E_{1}, m_{p}$ the proton mass and $\Delta t_{\text {system }}$ the overall resolution including the TOF detector and data acquisition system. Finally, the kinetic energy of the proton and the distance where the detector is placed are key parameters for a good temporal and energy resolution. A typical system resolution of a TOF diagnostic is generally around $\Delta t_{\text {system }} \approx 250$ picoseconds, which allows to expressed the minimum energy increment possible according to the incident kinetic energy of the proton $E_{1}$ and the position of the detector with respect to the target:

$$
\Delta E_{\text {min }}=\left[\frac{1}{\sqrt{E_{1}}}-\left(\frac{\Delta t_{\text {system }}}{\Delta x_{T O F}} \times \sqrt{\frac{2}{m_{p}}}\right)\right]^{-2}-E_{1},
$$

and is represented in Figure 3.20b

This method has some disadvantages such as a bad resolution for light ions or protons of several tens of MeV. Indeed, the time difference in the TOF signal between hard X-rays peak and ion/proton peak drastically decreases, thus it becomes difficult to distinguish both contributions. Placing the detector farther away from the target will result in a low density of particle reaching the detector and thus a lower signal readout, due to the high divergence of these particles.

\section{Micro Channel Plate}

The micro channel plate (MCP) is a fast high-gain amplifier for electrons originally developed for image intensification devices sensitive to charge particle and electromagnetic radiations with sufficient short wavelength. It has extended its application to other fields such as X-ray detection [Kellogg et al., 1976], medical application 


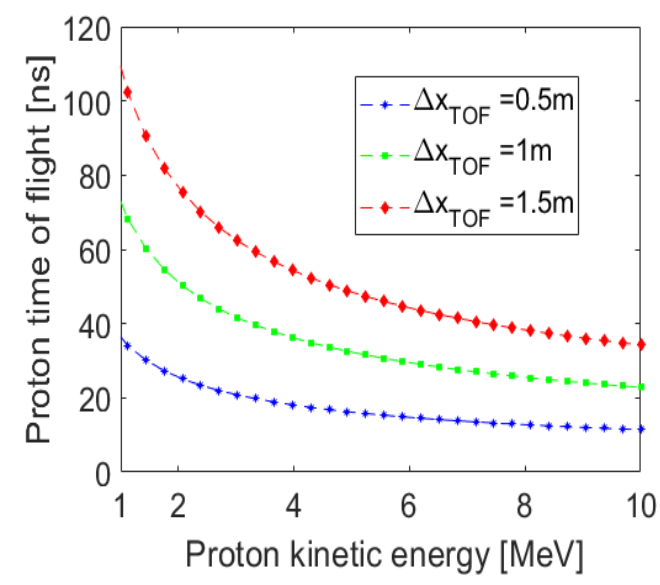

(a)

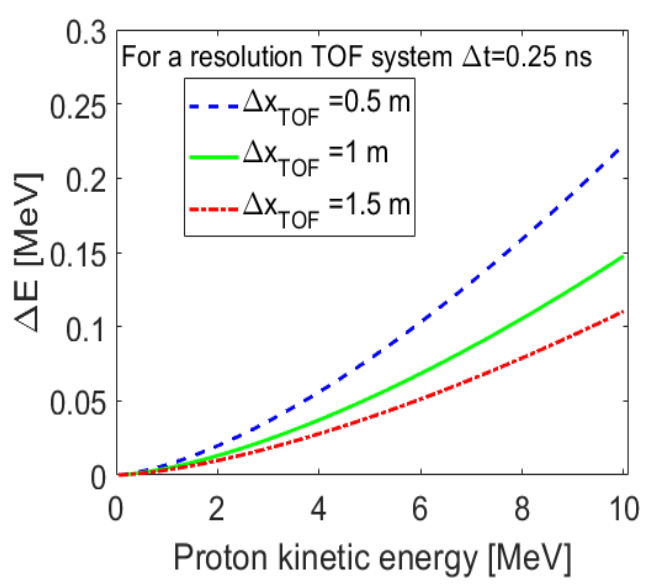

(b)

Figure 3.20: Energy and time resolution dependence of Time of Flight technique. a) Representation of the time needed for a proton with different kinetic energy to travel on a distance $\Delta x_{T O F}$. b) Energy step resolution $\Delta E=E_{2}-E_{1}$ of different proton kinetic energy $E_{1}$ read by a detector placed at a distance $\Delta x_{T O F}$ with 250 ns time resolution.

[Shikhaliev et al., 2004], industrial inspection and a time-of-flight technique. It is a circular plate with a thickness of about $1 \mathrm{~mm}$, which consists of an electrically insulating material of glass and containing an array of about $10^{4}-10^{7}$ tiny holes of mini electron multipliers (microchannels) acting separately and oriented parallel to each other (see Figure 3.21). The diameter of each channel varies usually between $10 \mu \mathrm{m}$ and $20 \mu \mathrm{m}$ depending on the resolution and are center-to-center spaced by approximately $15 \mu \mathrm{m}$. When the primary radiation impinges the microchannel wall, it creates secondary electrons. By applying a potential difference generally in the order of $1 \mathrm{kV}$, the secondary electrons are accelerated towards the channels end, creating further secondary electrons when colliding several times with the channel wall. Thus an electron avalanche is generated in the channel, and the signal is amplified depending on the electric field strength and the geometry of the micro-channel plate (see figure 3.21). After the cascade, the charge in each microchannel walls must be replenished and it may take some time to recharge before it can detect another signal. This dead time is given by the ratio of the output charge per channel over current per channel [Ladislas Wiza, 1979].

When the secondary electron cloud collides with residual gas atoms at the end of the channel, ions are produced. Such ions can drift back to the beginning of a straight channel, producing new pulses and a distorted signal. Ion feedback can be inhibited simply by curving with a small angle each microchannel but such a device is difficult to achieve in a single thin microchannel plate. By assembling 2 or 3 plates near each other depending on the application, the output gain can be considerably increased, 


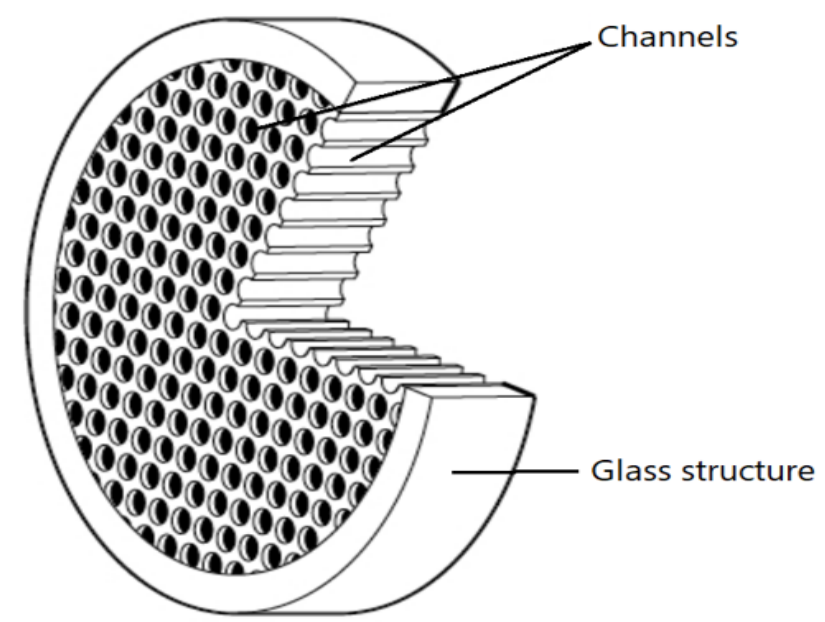

high voltage

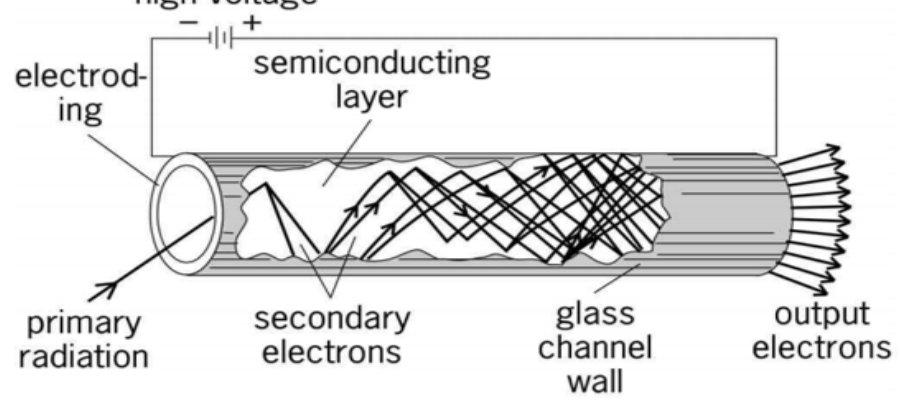

Figure 3.21: Working principle of a microchannel plate taken from [Ladislas Wiza, 1979]. An incoming particle hits the channel wall, creating two or more secondary electrons. By applying a voltage difference of about $1 \mathrm{kV}$ between the front and end of the channel, the electrons are accelerated towards the end, generating in turn further secondary electrons when colliding with the channel wall. Thus the signal from the initial particle will be amplified. 


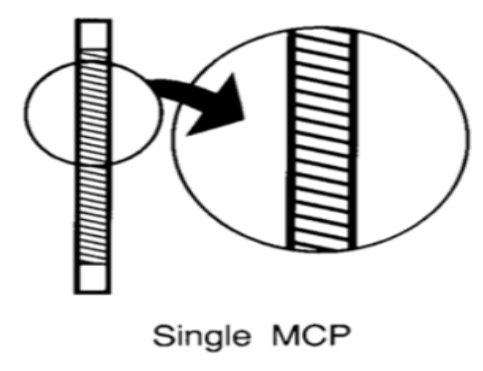

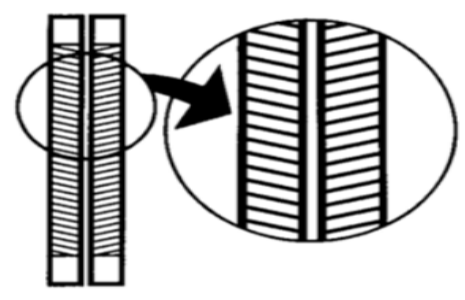

Chevron Assembly

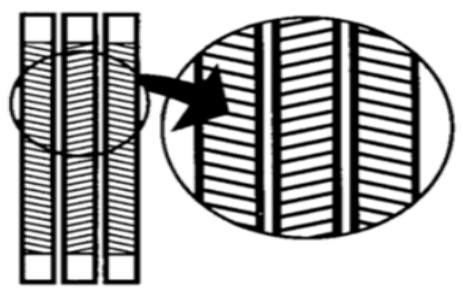

Z-Stack Assembly

Figure 3.22: MCP configurations (source: Galileo ElectroOptics Corporation)

such as in the MCP Chevron or Z-stack assembly, described in Figure 3.22.

The channels of each MCP are usually tilted $\left(8^{\circ}-15^{\circ}\right)$ against the MCP input surface and the channels of successive MCPs are tilted to opposite directions. However, in some applications the spatial information of the detected beam has to be preserved. In that case a phosphor screen is placed behind the MCP to produce an image, which in turn is detected by a CCD camera.

Semiconductor photodiodes can also be used for TOF detection. The interested reader can refer to the Appendix A for more details on their operating mode.

\subsubsection{Magnetic spectrometer (Thomson Parabola spectrometer)}

The simplest method to study ion emission is to measure the time-of-flight (TOF) of the ions. However, in the presence of a multi-species and multi-energetic ion beam obtained from laser driven sources, TOF diagnostics become limited in resolution and won't be able to distinguish neither generation of different atomic species and their charge states. The Thomson Parabola spectrometer (TP) [Thomson, 1913] is known for its high capability of providing simultaneously the distribution of accelerated ions according to their kinetic energy and charge (Z)-to-mass(A) ratio. Based on the deflection of charge particle by electric and magnetic field, the TP enables to energy-resolved ions spectra for different charge-to-mass ratio. In a typical layout represented in Figure 3.23, a very small part of an ion beam is selected by the pinhole placed at the entrance of the TP. The pencil beam travels through a region of parallel magnetic $\left(\mathbf{B}_{0}\right)$ and electric field $\left(\mathbf{E}_{0}\right)$ applied along the $\mathrm{x}$-axis where it is deflected 


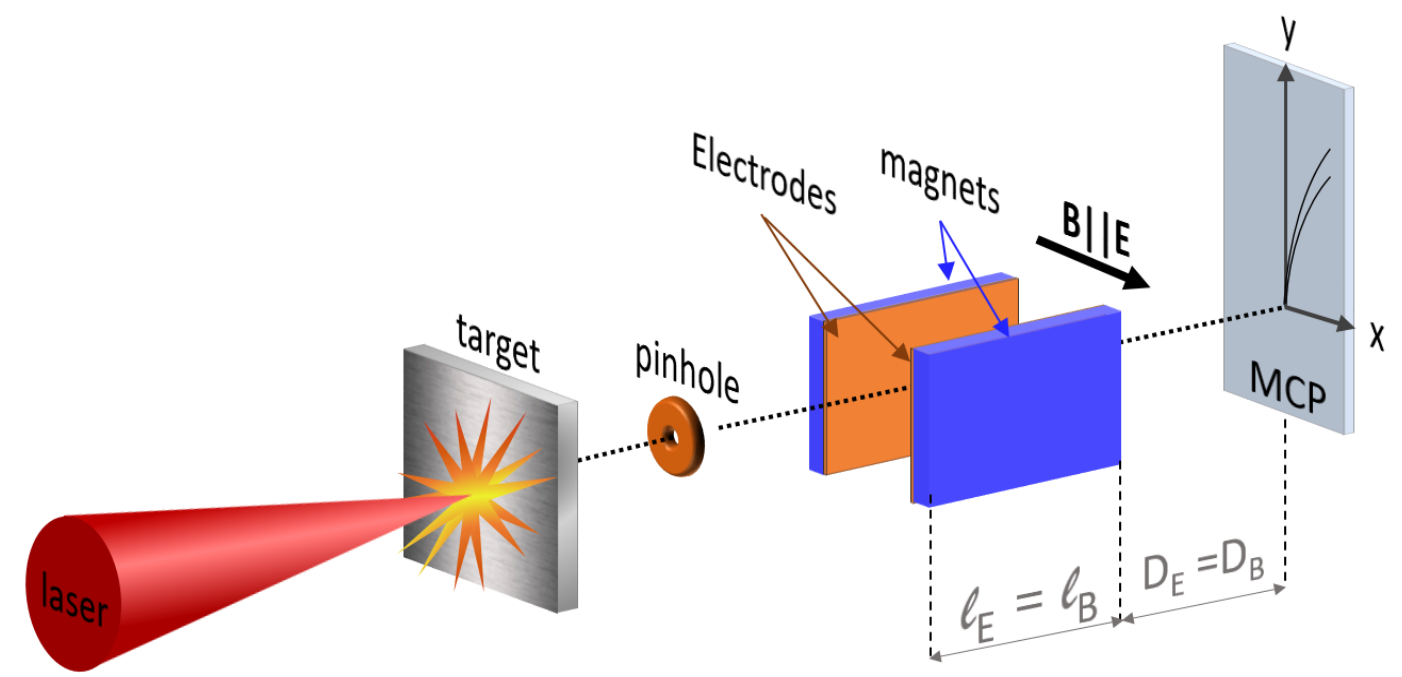

Figure 3.23: TP for the detection of laser-driven heavy ion beams. The incoming ion beams are deflected according to the Lorentz force (3.1) by two permanent magnets for the magnetic field and a pair of charged copper electrodes for the electric fields. The ion beams are separated by their charge-to-mass ratio along parabolic traces on the detecting material.

according to the Lorentz force (3.1) along the y-coordinate for the magnetic field and $\mathrm{x}$-coordinate for the electric field in a parabolic trace as represented in Figure 3.24. $\mathrm{X}$-rays, pass through the electric and magnetic field without any deflection, producing the so-called zero-point used as a reference. The TP developed at the CLPU facility was composed by a magnetic field of $0.2 \mathrm{~T}$ measured with a Hall probe, ensuring diagnostic in the 1-10 MeV ion energy range, with a resolution of $7 \mathrm{keV}$ for $1 \mathrm{Mev}$ and $100 \mathrm{keV}$ for $5 \mathrm{MeV}$. Two copper plates with adjustable high-voltage supplies up to $10000 \mathrm{~V}$, generally operating at $5000 \mathrm{~V}$ were used to generate the electric field. A $200 \mu \mathrm{m}$ aperture pinhole was placed at the entrance of the TP. Considering an ion beam travelling along the z-axis, with $E_{0}$ and $B_{0}$ fields oriented along the x-axis and assumed uniform, the displacement of the ions on the detector plane along the $\mathrm{x}$ (due to $E_{0}$ field) and y (due to $B_{0}$ field) axis can be derived analytically as the following:

$$
\begin{aligned}
& x_{\text {elec }}=\frac{q E_{0}}{m v^{2}} \ell_{E}\left(\frac{1}{2} \ell_{E}+D_{E}\right), \\
& y_{m a g}=\frac{q B_{0}}{m v} \ell_{B}\left(\frac{1}{2} \ell_{B}+D_{B}\right),
\end{aligned}
$$

where $q$ is the ion charge, $m$ its mass and $\mathrm{v}$ its velocity along the $\mathrm{z}$ axis. $E_{0}$ and $B_{0}$ are the electric and magnetic fields, $\ell_{E}$ and $\ell_{B}$ there length, $D_{E}$ and $D_{B}$ the distances between the end of the fields and the detector plan. The parabolic deflection of a 


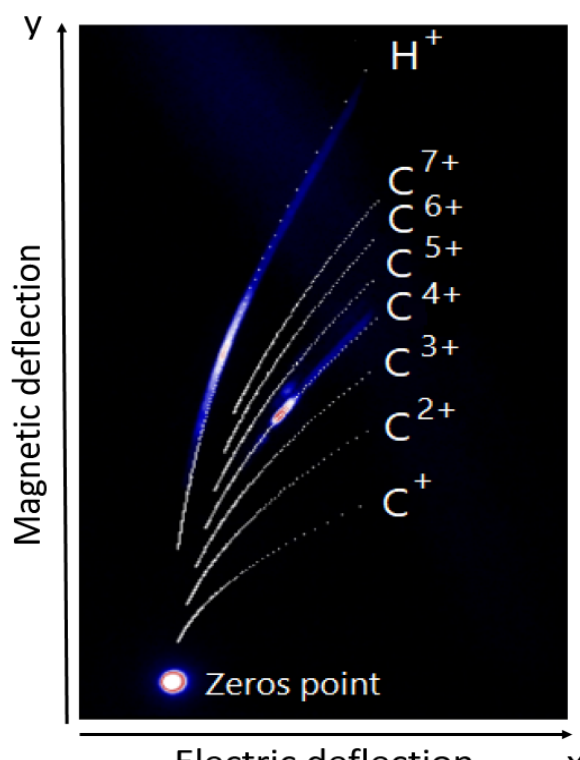

Electric deflection $\quad \mathrm{x}$

Figure 3.24: Traces of carbon ions and protons. The no deflected circular and bright signal is considered as the zero-point and is mainly due to x-rays. Ion with higher energy will be the ones closer to the zero-point.

given particle $q / m$ can be obtained inserting (3.84) into (3.85) by eliminating the velocity $v$.

$$
y_{m a g}^{2}=\frac{q B_{0}^{2}}{m E_{0}} \frac{\ell_{B}^{2}}{\ell_{E}} \frac{\left(\frac{1}{2} \ell_{B}+D_{B}\right)^{2}}{\left(\frac{1}{2} L_{E}+D_{E}\right)} x_{e l e c}
$$

By considering non-relativistic case, the ion kinetic energy $E_{k i n}=1 / 2 m_{p} v^{2}$ can be expressed according to the magnetic field deflection by inserting it into equation (3.86) and eliminating $x_{\text {elec }}$ :

$$
E_{k i n}=\frac{\left(q B_{0} L_{B}\right)^{2}}{2 m} \frac{\left(\frac{1}{2} L_{B}+D_{B}\right)^{2}}{y_{m a g}^{2}} .
$$

However, the trace starts to be indiscernible at high energies. In principle, the deflection of ions by the magnetic field is not limited in space since the displacement is orthogonal to the field direction meanwhile the electric deflection is directly dependant on the inter-space between the two electrode plates. Since the positively charged ions are deflected towards the negative electrode, the TP and pinhole are aligned so that the incident particle beam is close as possible to the positive electrode in order to allow maximum deflection. 


\subsubsection{Passive Ion sensitive detectors}

Passive detectors offer several advantages over alternative charge particle detector such as time-of-flight detectors [Wolff \& Stephens, 1953]. They don't depend on any electrical circuit and are therefore insensitive to eventual electromagnetic pulses that are generated during high-intensity laser-plasma interaction experiments[Brown et al., 2008; Poyé et al., 2015].

\section{Cr39}

Columbia Resin Number 39 or CR-39, is a solid-state nuclear track detectors composed by allyl diglycol carbonate polymer, with a density of $1.3 \mathrm{~g} / \mathrm{cm}^{3}$. It is used extensively in laser-driven ion acceleration experiments to record directly the absolute number of ions. Indeed, in addition to be insensitive to EMP, the detector is largely immune to x-rays and electrons commonly produced in laser-matter interaction experiments, and highly sensitive to proton and ion particles. When a ion particle or proton interacts with the polymer structure, it leaves a path of broken chemical bonds within the CR-39. After irradiation, these tracks (pits) are revealed by etching process and enlarged with etching time (see Figure 3.25). The number of holes corresponds to the absolute number of incoming ions and can be counted with standard optical microscope techniques, making it usefull for flux measurements. Also, by measuring the diameter of the trail, a measure of the particle charge specie and incident energy can be deduced [Fleischer et al., 1965; Sinenian et al., 2011]. Cr-39 is etched with 6.5 mol of a Sodium Hydroxyde solution $(\mathrm{NaOH})$ kept at $\sim 85^{\circ} \mathrm{C}$ [Scullion et al., 2016] with a high precision thermal bath (see Figure 3.25). The etching time usually variates from few minutes up to 1 hour according to the particle's density and pits size. An overetching will cause a saturation in the CR-39, making pits overlapping and becoming indistinguishable [Gaillard et al., 2007]. In addition to be time-consuming diagnostic, the CR-39 has the disadvantage to be unusable after once, which does not make it the most suitable detector. However, CR-39 can be used in parallel with other detectors like radiochromic films, Thomson Parabola, or images plates [Prasad et al., 2010] to give an absolute calibration of the number of incident ions.

\section{Radiochromic films}

Radiochromic films (RCF) are commonly used in stack configuration to diagnose particle beam dose deposition in Industry[Aea-Tecdoc-1070, 1999; ISO/ASTM51275, 2013] and Medicine[Butson et al., 2003]. More recently, they have also proven to be a reliable time-integrated energy spectrum diagnostic [Feng et al., 2018; Green et al., 2014; Schollmeier et al., 2014] of proton beams produced by TNSA [Gitomer et al., 1986; Snavely et al., 2000]. Apart from measuring the absorbed dose, the RCF films also inform on the spatial profile of the particle beams, allowing detailed characterization of the particle dynamics. In most experiments report in this thesis, the Gafchromic ${ }^{\circledR}$ film HD-V2 is used. HD-V2 films are produced by Ashland company and consist of an active layer, nominally $12 \mu \mathrm{m}$ thick, containing the active compo- 

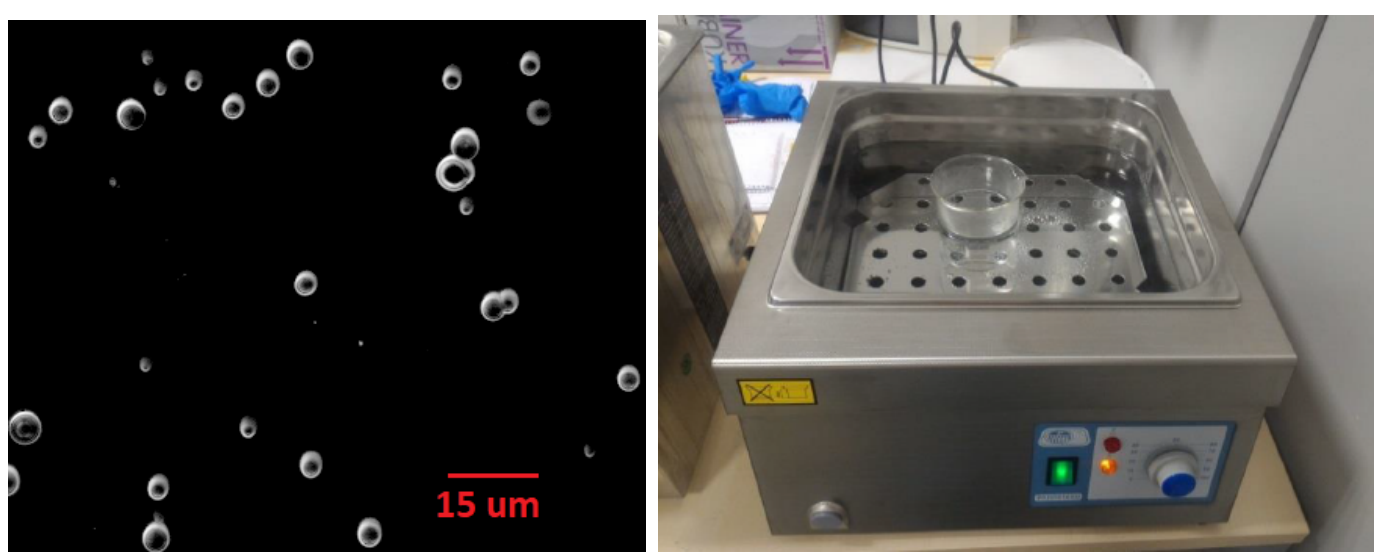

Figure 3.25: Left: image of a Cr39 obtain with electronic microscope x50 magnification and dark filter after irradiation by ions beams. Right: high precision thermal bath for etching process.

Table 3.1: Atomic composition of HD-V2 film layers

\begin{tabular}{|c|c|c|c|c|c|c|c|c|c|c|}
\hline \multirow[t]{2}{*}{ Layer } & \multirow{2}{*}{$\begin{array}{c}\text { Thickness } \\
{[\mu \mathrm{m}]}\end{array}$} & \multirow{2}{*}{$\begin{array}{l}\text { Density } \\
{\left[\mathrm{g} / \mathrm{cm}^{3}\right]}\end{array}$} & \multicolumn{8}{|c|}{ Composition by atomic [\%] } \\
\hline & & & $\mathrm{H}$ & $\mathrm{Li}$ & $\mathrm{C}$ & $\mathrm{N}$ & $\mathrm{O}$ & $\mathrm{Na}$ & $\mathrm{Cl}$ & $\mathrm{Al}$ \\
\hline Active & 12 & 1.2 & 58.4 & 0.6 & 27.9 & 0.1 & 11.7 & 0.5 & 0.6 & 0.3 \\
\hline Polyester Substrate & 97 & 1.35 & 36.4 & / & 45.5 & / & 18.2 & / & / & / \\
\hline
\end{tabular}

nent, marker dye, stabilizers and other components giving the film its dose response. The thickness of the active layer may vary slightly. The active layer is coated on a clear, $97 \mu \mathrm{m}$ Polyester substrate. Both material compositions are specified in Table 3.1. HD-V2 films turn blue when irradiated by particles and become darkener with increasing absorbed doses. It develops itself in real time without any post-exposure treatment. The active structure has a very small scale response allowing extremely high resolutions dose profile in addition to a spatial resolution less than $5 \mu \mathrm{m}$. According to the manufacturer, the dose working range of HDV2 is in between 10 and 1000 Gy [Gafchromic, 2020] and can cover a wide energy deposition range from few hundreds of $\mathrm{KeV}$ up to few tens of $\mathrm{MeV}$. Doses lower than $10 \mathrm{~Gy}$ lead to non-measurable active layer darkening while doses greater than 1000 Gy saturate the HDV2 active layer dose response. A scanning process is needed after exposure in order to establish the correspondence between the dose deposited by the charged particle and the pixel intensity of scanned irradiated HD-V2 layers images. For a precise measurement of the absorbed dose, a calibration measurement with a well-known dose is required. 

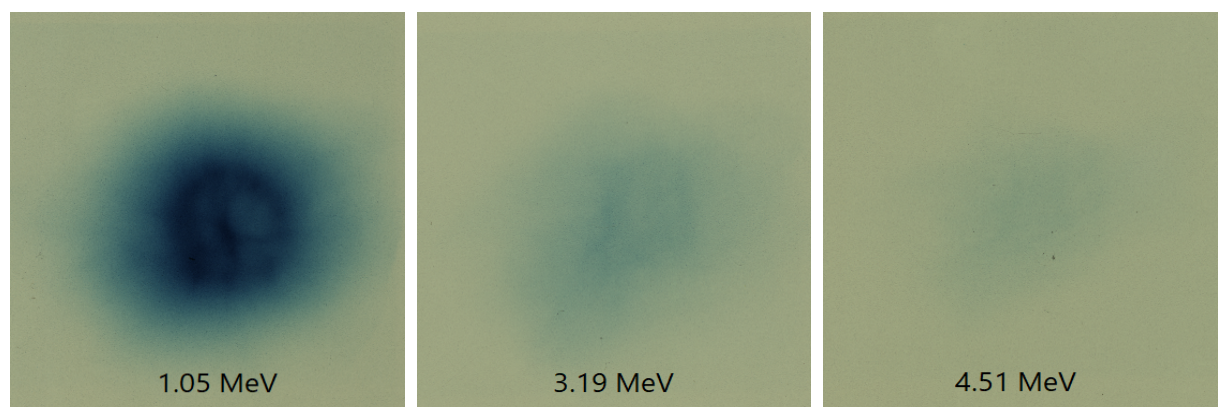

Figure 3.26: Proton beam detection with Gafchromic Film HD-V2 - Sample ion beam detected with a RCF stack. The layers correspond to diffrent energies according to their attenuation curves

\subsubsection{Toward active diagnostics: Fast scintillators}

When a charged atomic or nuclear particle interacts with matter, it dissipates its energy through ionization and excitation of the molecules of the medium and will produced visible radiation, known as fluorescence. The main instruments developed for the detection of these particles are based on this general process. The scintillation counter is one of these instruments, which consists in the detection of the fluorescence emission produced by an ionizing particle and has been invaluable in many studies. It allowed a direct method of particle counting and has been considerably used in the research about $\alpha$ rays [Crookes, 1902] but had also been a support in the discovery of complex atom structure [Rutherford, 1911] and the comprehension of radio-active elements.

According to Knoll [Knoll, 2010], a suitable scintillator should respect the following conditions:

1. High efficiency conversion of charged particle kinetic energy into detectable light.

2. A linear response of the scintillator according to deposited energy.

3. Transparency of the medium to not absorb the wavelength of its emission.

4. A short decay time of the induce luminescence for fast signal measurements.

5. Possibility of manufacturing variable sizes of scintillator for different kind of applications and be composed by a high optical quality material.

6. A refractive index that permits an efficient transport of scintillation light to the detector.

The development of scintillators that fulfills most of these conditions is very challenging due to the requirements concerning the nature of the particles to detect as well as the domain of applications. The scintillators can be range into two groups according to their chemical composition and scintillation process; the inorganic where the most widely used are alkali halides, among others the sodium iodide (NaI). This type of scintillator tends to have a high light output (luminescence) compared to 
organic scintillators but quite slow in the scintillation response. Organic scintillators are generally separated into 3 categories; crystal, liquids and plastics, where the luminescence depends essentially on the molecular properties of the medium, and tends to have a lower light yield but faster response. The latter will be the main subject of this section.

The naphthalene block, first organic scintillator to be used, by Broser and Kallmann in 1947 was a major advance in the counting technology development [Broser \& Kallmann, 1947]. In the next years, many other organic scintillators more efficient were developed such as the crystalline anthracene [Bell, 1948], liquid scintillators [Ageno et al., 1950; Kallmann, 1950; Reynolds et al., 1950] and plastic scintillators [Schorr \& Torney, 1950]. Nowadays, polyvinyl-toluene (PVT) and Polystyrene (PS) are the most widely used base for plastic scintillator detectors and make them a very low price product compare to crystalline scintillators. They show a high sensitivity for charged particles, energy deposition and are able to detect single ions [Smith et al., 1968]. The research of plastic scintillators has been increased for application as radiotherapy dosimetry due to their water equivalent composition, large output light and short decay time (ns scale) which also make them suitable for fast timing measurement profile applications.

\section{Polyvinyl Toluene scintillators}

A common feature to most organic scintillators is the benzene ring, characterized by the delocalisation of three $\pi$ bonds, which form the basis for the luminescent properties of organic scintillators (see Figure 3.27). In the case of the Polyvinyl Toluene (PVT) scintillator, the benzen ring is bonded to a methyl $\left(\mathrm{CH}_{3}\right)$ and vinyl group $\left(\mathrm{CH}_{2}=\mathrm{CH}\right)$. Unlike the majority of inorganic compounds, these organic molecules are loosely bound together by Van der Waals forces instead of existing in a well ordered crystal matrix and bound together with covalent bonds.

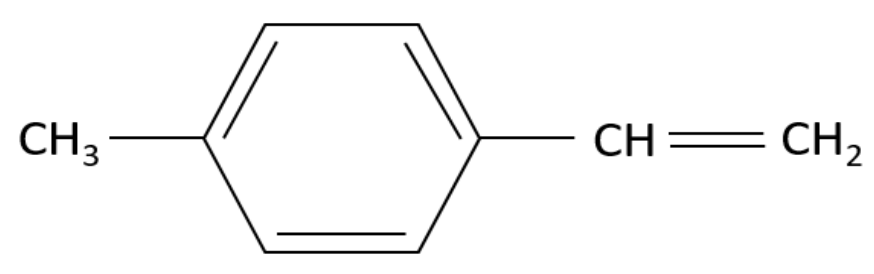

Figure 3.27: Polyvinyl Toluene molecule presents in PVT plastic scintillators.

A PVT BC-400 plastic scintillator has been used in the experiments within the scope of this thesis and a detailed study will be presented in Section 4.4.

\section{The scintillation mechanism in organic scintillator}

The $\pi$-electronic energy levels of an aromatic hydrocarbons form the basis for the luminescent properties of organic scintillators and are illustrated in Figure 3.28. The 
levels are divided into a sequence of singlet states (spin 0) $S_{i}$ and triplet states (spin 1) $T_{i}$ with $\mathrm{i}=1,2,3 \ldots$ corresponding to excited singlets and triplet states and $S_{0}$ the singlet ground state. Inside each of these electronic levels are superimposed vibrational sub-levels, where $S_{00}$ represents the lowest vibrational state of the ground electronic state.

The luminescence of simple organic molecules is divided in three parts:

-The fluorescence

-The phosphorescence

-The delayed fluorescence

For most applications, fluorescence is the principal process used to perform precise timing measurements since it is a fast scale process with a decay time of typically few nanoseconds. It consists of a radiative transition from the $S_{10}$ singlet state to a vibrational state of the ground singlet state $S_{0}$, following absorption. The fluorescence intensity $I$ decays exponentially with the time $t$ such as

$$
I \approx I_{0} \exp (-t / \tau)
$$

with $I_{0}$ the intensity at $\mathrm{t}=0$ and $\tau$ the fluorescence decay time.

Some fraction of molecules excited into a singlet state $S_{1}$ may have a radiationless transition through an intersystem crossing process into an excited triplet state $T_{1}$. The triplet state lifetime usually excesses $10^{-3}$ sec. The radiative transition from $T_{1}$ to $S_{0}$ corresponds to the phosphorescence process. Since the excited $T_{1}$ state is energetically lower than the $S_{1}$ state, the wavelength of the phosphorescence spectrum is longer than the fluorescence one. When the $\pi$-electrons acquire enough thermal energy from a $T_{1}$ or in some other metastable state $\mathrm{M}$ to return to the $S_{1}$ state, they decay through a delay fluorescence process, with a decay time that increase up to $10^{-6} \mathrm{sec}$ or longer depending on the lifetime state, the temperature or the $T_{1}-S_{0} /$ $M-S_{0}$ energy gap, but with the same wavelength than the fast fluorescence process. Since all the fluorescence radiation emissions within a vibrational sub-level of the ground state $S_{0}$ have a lower energy than the minimum required for excitation, a very small overlap between the absorption and emission spectrum will occur, which may self-absorb a little part of the emission spectra fluorescence. The phenomenon is known as Stokes shift. The scintillator efficiency depends on the one hand of its fabrication method as well as its constituents and on the other hand on the particle species and their kinetic energy. It is defined as a fraction of the converted incident particle energy into photon. This efficiency is altered by the de-excitation mode of the excited molecules which are radiativeless transitions, corresponding mainly to heat. Such process is known as quenching effect.

\section{Quenching effect and Birks Law}

The physical mechanism behind the quenching phenomenon are not yet fully understood, and regroup different processes. Among other, the concentration quenching can be explained as a formation of excited dimers from the resonance interaction between an excited and unexcited molecule [Birks, 1964]. The excited dimers may be 


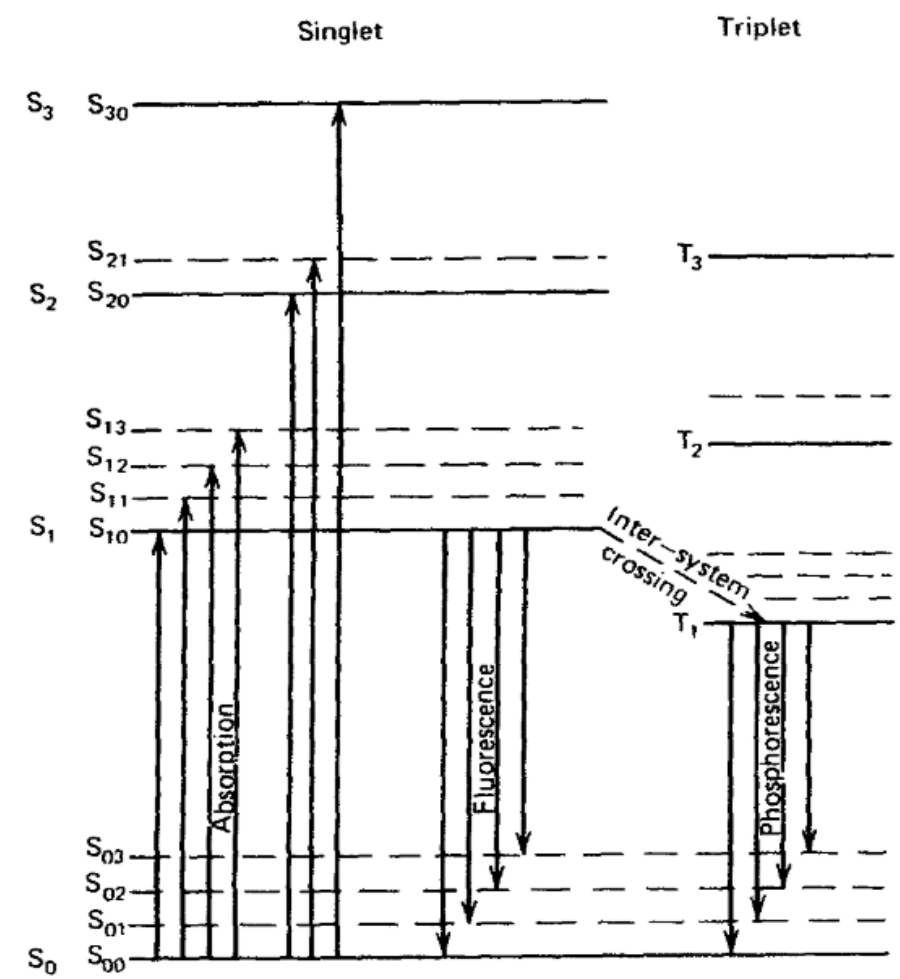

Figure 3.28: Energy levels of a $\pi$-electron system in an organic molecule from [Knoll, 2010].

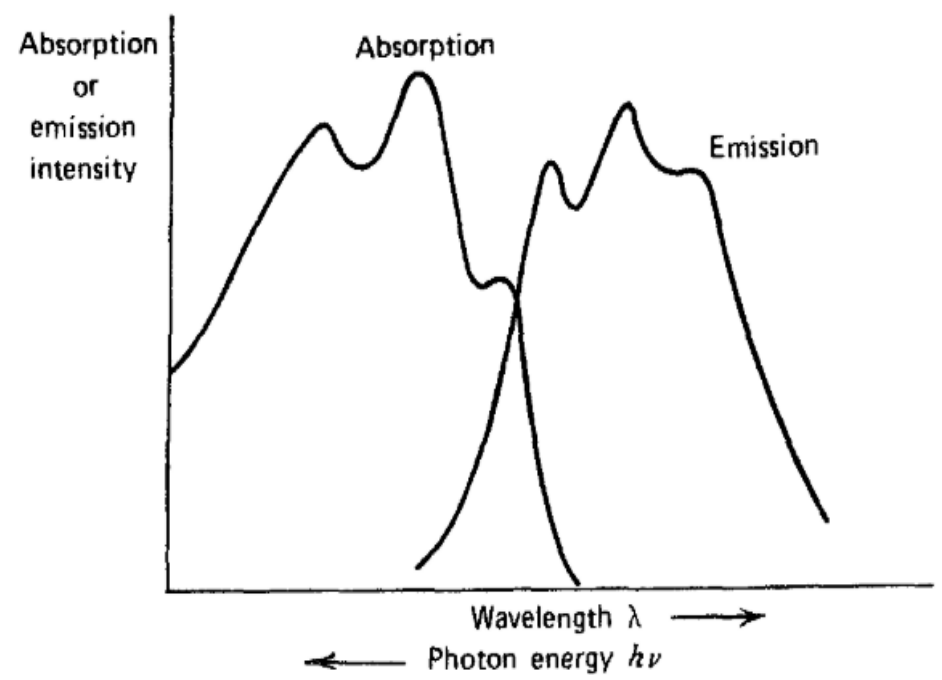

Figure 3.29: Absorption and emission spectrum for an organic scintillator, from [Knoll, 2010]. 
luminescent or non-luminescent, and either stable or unstable. This leads generally to a thermal degradation of the energy and thus a drop in light yield. The ionization quenching process, common to all organic scintillators, affects the intensity of the fast scintillation component when interacting with heavy charge particle as proton or alpha particle. Indeed, organic scintillators composed by anthracene, commercial plastic scintillators and other kind, have a linear response to electrons above $125 \mathrm{keV}$ [Brannen \& Olde, 1962]. The response to heavy charged particle compared to electron is always less for same incident kinetic energies, with a non linearity above much higher energy as represented in Figure 3.30. Considering the BC-400 model, the light output response to few hundreds of $\mathrm{keV}$ is about 10 times lower for proton than for electron [Knoll, 2010] and 100 time lower for alphas particles with respect to electron response.

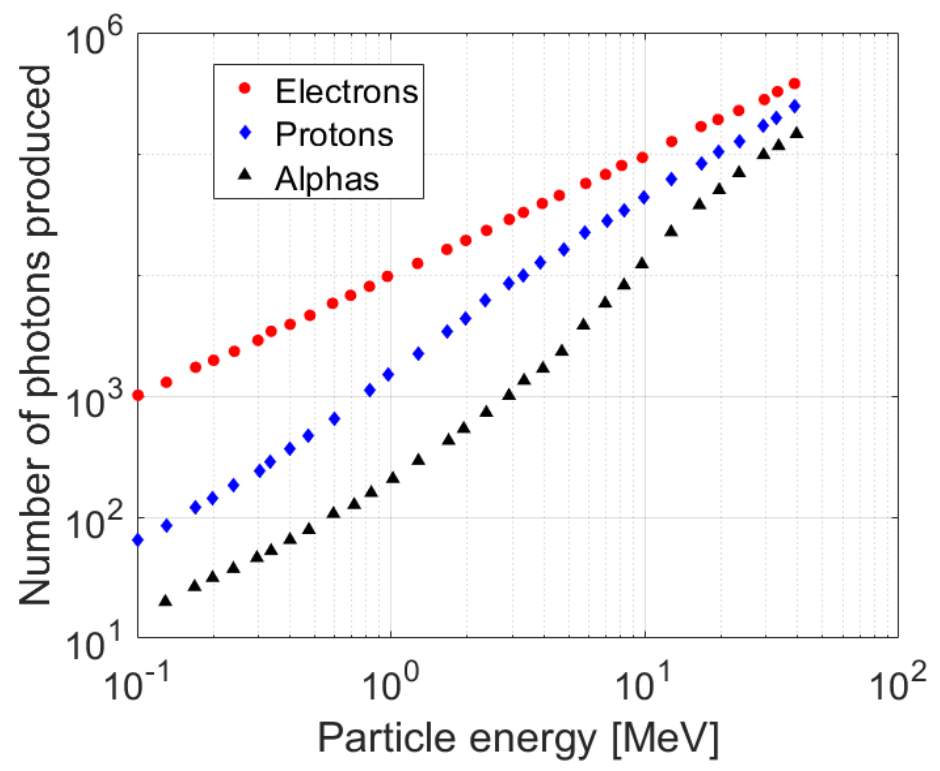

Figure 3.30: Light output response of the plastic scintillator BC-400 to different particles from $100 \mathrm{keV}$ to $40 \mathrm{MeV}$. Graph reformed from [Saint-Gobain-Crystals, 2020].

Birks [Birks, 1951] proposed a good model to describe the Ionization quenching by describing the relation between the fluorescent emission per unit path $d L / d z$ [photons $\mathrm{cm}^{-1}$ ] (with $z$ the propagation axis), and the energy loss of the particle per unit path $\left[\mathrm{MeV} \mathrm{cm}^{-1}\right]$. His method assumes that high ionization density along the particle track leads to quenching effect from damaged molecules, which tends to dissipate the ionization energy non-radiatively. This process is strongly observed for protons and other heavy ionizing particles, thus lowering the scintillation efficiency. If we assume that the local concentration of damaged molecules along the particle path is proportional to the energy loss, we can note $B d E / d z$ the ratio between un-damaged and 
damaged molecules, where $B$ is a constant. If we account for the relative quenching probability $k$, thus the fluorescence per unit path reads:

$$
\frac{d L}{d z}=\frac{A \frac{d E}{d z}}{1+k B \frac{d E}{d z}},
$$

and is known as the Birk's formula, where $A$ is the scintillation efficiency, corresponding to the fraction of energy absorbed by the scintillator and converted into light [photons $\mathrm{MeV}^{-1}$ ], $k$ and $B$ are treated as a single parameter specific to each scintillator model $k B\left[\mathrm{MeV}^{-1} \mathrm{~cm}\right]$. At small $d E / d z$ such as for fast electrons with sufficient large kinetic energy, Birk's formula becomes

$$
\frac{d L}{d z}=A \frac{d E}{d z}
$$

where a linear relation between the emitted fluorescence per unit path and the stopping power is observed. For large $d E / d z$, creating saturation on the track, such as for alpha-particle or proton, equation (3.89) becomes

$$
\frac{d L}{d z}=\frac{A}{k B},
$$

with a response that tends towards a constant. The parameter $\mathrm{kB}$ is deduced from the fit of the model to the experimental data and is specific of each scintillator composition and doesn't depend on the nature of the particle, in theory.

The decrease of scintillation efficiency and non-linear response associated to heavy particles limits the utility of organic scintillators for direct spectrometry of heavy particles and requires a calibration of the energy response for absolute measurements. 


\section{Chapter 4}

\section{A 2D scintillator-based proton detector for high repetition rate experiments}

The CLPU is a multi-Terawatt laser facility with the VEGA laser pulse line working at a shot sequence up to $10 \mathrm{~Hz}$. A careful development of repetition rate based particle diagnostics is therefore essential to properly carry out experiments with the VEGA laser system. Interest in scintillation-based diagnostics is growing in the community studying ultra-short laser accelerated particles. Indeed, this type of diagnostic is capable of being set in the high repetition rate (HRR) mode while maintaining the characteristics of conventional passive diagnostics. This chapter is dedicated to the theoretical and experimental study of a scintillator-based detector able to measure the proton energy and the spatial distribution. After having briefly presented the latest advances of scintillator-based diagnostics in the laser-plasma physic community, we will focus on the prototype developed and built at the CLPU. Then will be presented the first test of the detector, done at the proton accelerator of the Centro de Micro-Análisis de Materiales located in Madrid (CMAM) where the scintillator response was characterized. Finally, we will introduce the first implementation of the detector in a laser-driven proton experiment, carried out at the Helmholtz-Zentrum Dresden-Rossendorf located in Dresden (HZDR), giving very promising results for this new class of on-line detectors in laser-plasma physic experiments.

\subsection{Context and recent developments}

We have seen in the previous chapter that the spatial compactness of the laserdriven proton acceleration technique combined with the high brightness particle, high beam laminarity and short particle pulse makes the laser-driven proton source potentially interesting for several applications. The energy spectrum of laser-driven proton beams, however, is typical very broad (tens of $\mathrm{MeV}$ ) and the divergence angle of the 
a

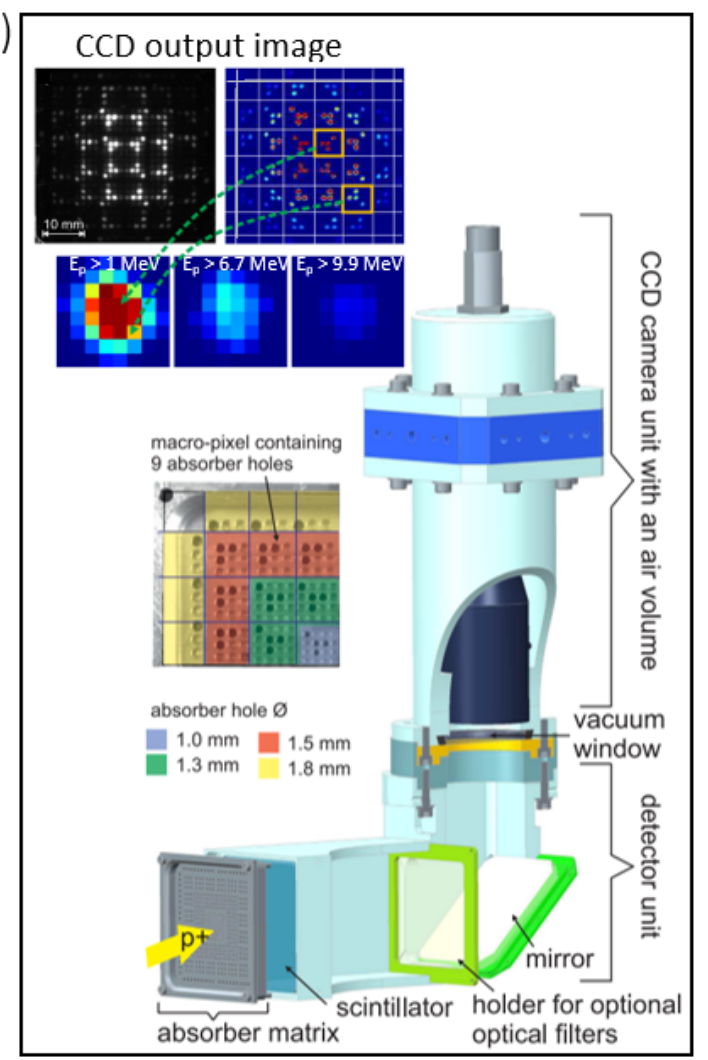

b)

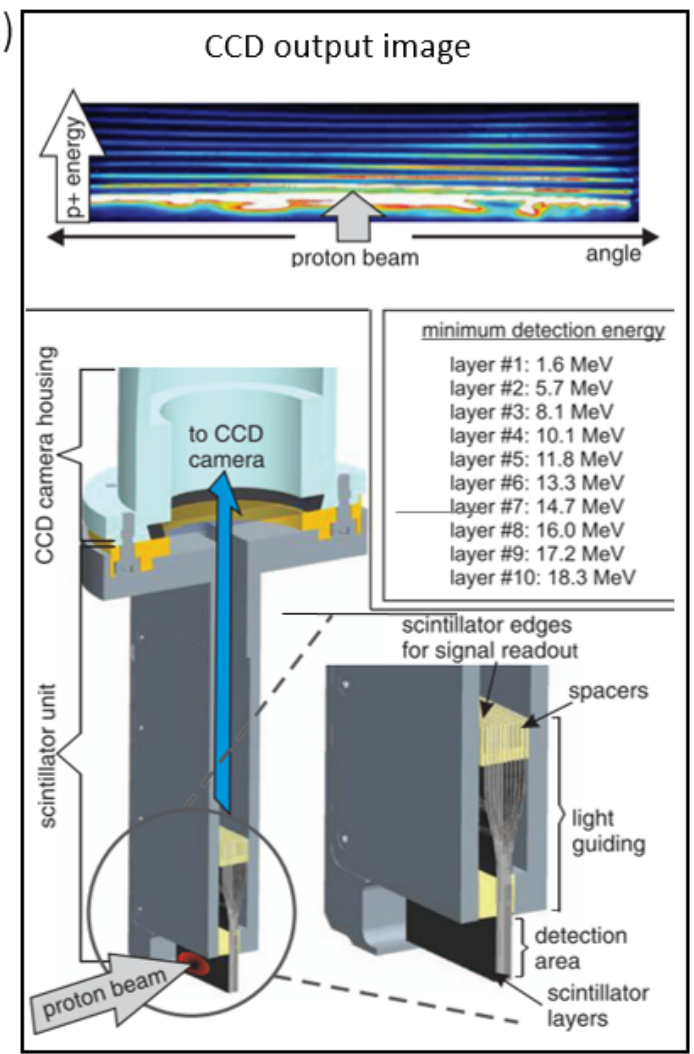

Figure 4.1: Scintillator detector from the team of HZDR laboratory. a) Detector design from the team of [Metzkes et al., 2016]. Protons interact with the different absorber holes of the matrix and deposit their specific energies in the thin plastic scintillator placed behind the different aluminum filters. The interaction produces the emission of scintillation imaged onto a CCD camera. The absorber plate is segmented into 60 macro-pixels, each of them resolving up to 9 different threshold energies by the use of different absorber holes made by different aluminum thicknesses. Image taken from [Metzkes et al., 2016]. b) Scintillator detector from [Metzkes et al., 2012]. The protons impinge the detection area composed by a stack of 10 thin plastic scintillators and deposit their energy in each individual layer. The produced scintillation light of each scintillator is then guided toward the upper edge and collected by a CCD camera. Illustration from [Metzkes et al., 2012].

proton distribution is usually up to $20^{\circ}$ half angle and energy dependent. Still many studies are carried out to understand the process behind this acceleration mechanism through the characterization of the spectral and spatial properties of the proton beam. With the advent of high power lasers working at HRR, this task has been greatly enhanced, allowing an online shot-to-shot control and optimization of the accelerated proton beam. One of the most used diagnostic in laser-driven proton production 
consists of a series of Radiochromic films ( $\mathrm{RCF}$ ) placed one after each other and able to recover the proton number and spatial distribution as a function of the proton energy. However, this diagnostic needs to be extracted from the vacuum chamber in which the experiment is performed in order to execute the data analysis, making it inappropriate when a direct feedback of the results at HRR is needed. The possibility to extend this technique to HRR mode of operation is nowadays a challenge in the laser plasma community and several laboratories and research groups are working on this. The main idea is to substitute the active RCF layers with scintillator layers capable of converting the proton energy deposition into light that can be then collected by a CCD camera. Different approachs have been recently proposed to imitate the RCF stack but, up to now, only a partial extension of the RCF capabilities was possible. The group from the Rutherford Appleton Laboratory (RAL) [Green et al., 2011] designed a detector able to resolve simultaneously the proton beam angular profile at three different energy ranges. The detector consists of stack of three plastic scintillators emitting at different wavelengths imaged onto a color CCD camera from the rear side. This approach allows a high $2 \mathrm{D}$ spatial but a limited energy resolution. The group from HZDR [Metzkes et al., 2012] proposed a detector composed by a stack of ten thin plastic scintillators placed one after each other, as the RCF stack, with a readout system looking at the transversal scintillation emission. This design gives online access to the angularly resolved proton distribution along one dimension for 10 different energy ranges (see Figure 4.1). This approach improves the energy resolution at the cost of the two-dimensional spatial resolution. Again, the HZDR facility [Metzkes et al., 2016] developed another scintillator detector able to record a two-dimensional imprint of the proton beam. It is composed by 60 macro-pixel containing each of them few absorber holes of different aluminum thickness, providing up to 9 threshold energies. This prototype gives a good energy resolution but still limited in spatial resolution by the number and dimension of absorber holes (see Figure 4.1).

\subsection{A Full 2D-scintillator-based proton detector}

We present a scintillator-based detector able to measure both the proton energy and its transversal spatial distribution along the propagation axis and capable of being used at HRR. It consists of a series of scintillators placed similarly as an RCF stack but positioned with a relative angle one respect to the others in order to leave a free field of view for an imaging system looking at the back side of each layer. The imaging system can be arranged depending on the spatial condition. Each scintillator layer is covered on the front side by an aluminum foil to protect it from the light emission from the previous scintillator layer. The relative angle $\phi$ between each layer is the key factor in the design because it permits the acquisition of the full $2 \mathrm{D}$ proton distribution for each of the layers composing the stack. It is a relevant parameter because the total size of the detector depends critically on it. Increasing the angle will increase the total length of the detector which consequently will require to increase 
the layer dimension to detect a given proton emission solid angle at a farther distance from the source. To assess in detail, we assume a proton beam propagating in a

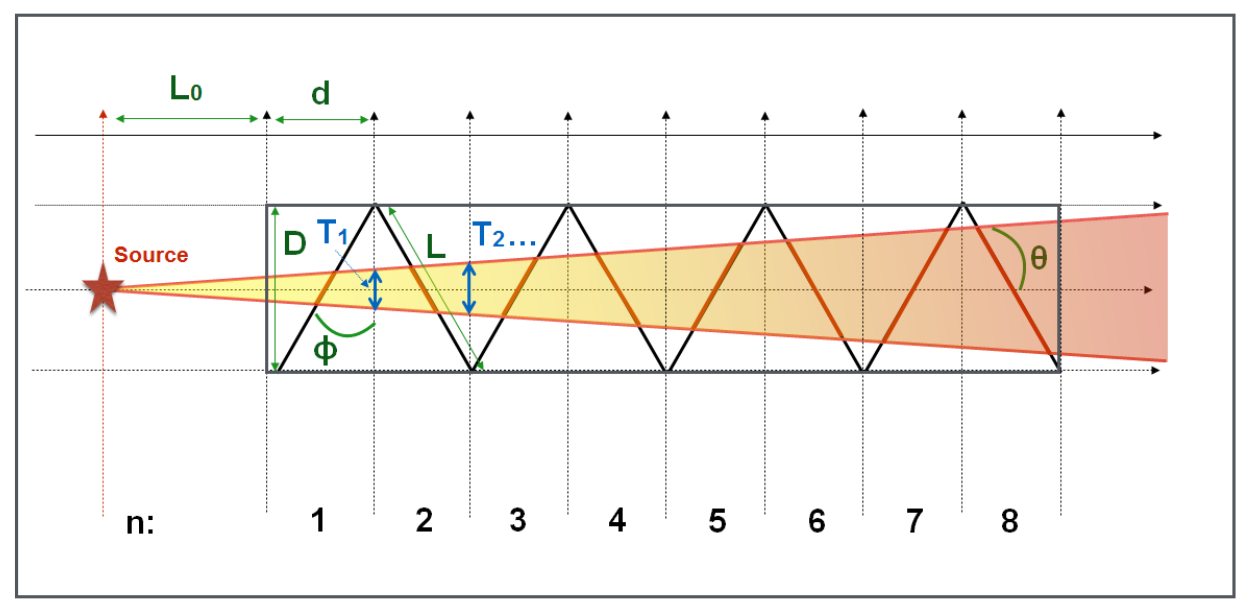

Figure 4.2: 2D top view of detector; the proton beam solid angle is parametrised by the internal half angle $\theta$, the detector dimension $\mathrm{D}$ is represented by the length of the scintillator layer $\mathrm{L}$, and the relative half angle between the layers $\phi, \mathrm{n}$ is the number of layers. $L_{0}$ is the distance between the proton source and the detector, $\mathrm{d}$ the longitudinal dimension of the scintillator layer and $T_{1} \ldots T_{n}$ represent the projection of the proton beam solid angle for each layer.

symmetric cone emission with half angle $\theta$ (see fig.4.2), where the transversal (D) and longitudinal (d) dimension of each scintillator layer (which are assumed to be all equal in size) perpendicular to the proton beam direction can be written as:

$$
D=L \cos (\phi) ; d=L \sin (\phi)
$$

The projection of the proton emission cone in the scintillator layer can be written as:

$$
\begin{aligned}
& T_{n}(\theta, \phi)=2 L_{n}(\phi) \tan (\theta) \\
& L_{n}(\phi)=L_{0}+n L \sin (\phi)
\end{aligned}
$$

$L_{n}$ is the effective length of the detector considered from the proton source emission. Let's note that $L_{0}$ must be greater than zero because a minimum distance between the detector and the source must be allowed for letting the possibility to host a magnet to deflect the electrons generated in the interaction process so as not to affect the scintillation signal. Finally $L_{n}$ depends on the angle between two successive layers $\phi$, on the dimension $L$ and on the number $n$ of the scintillator foils.

The working condition can be written as:

$$
D>T_{n}(\theta, \phi),
$$


where the size of the transverse projection of the scintillator D must be larger than the projection of the proton solid angle $T_{n}$. This can be solved as:

$$
\begin{aligned}
& n<n_{0}(\theta, \phi)+n^{\prime}\left(L_{0}, L, \phi\right) \\
& n_{0}(\theta, \phi)=\frac{1}{2 \tan [\theta \tan \phi]} \\
& n^{\prime}\left(L_{0}, L, \phi\right)=-\frac{L_{0}}{L} \frac{1}{\sin \phi}
\end{aligned}
$$

Where $n_{0}=n\left(\theta, \phi, L_{0}=0, L\right)$.

\subsection{1 case $L_{0}=0$}

The case $L_{0}=0$ corresponds to the assumption that the proton source is just placed in the surface corresponding to $\mathrm{n}=0$, so the system becomes:

$$
n<n_{0}(\theta, \phi)
$$

Equation 4.5 can be studied as a function of $\phi$ (for a given value of $\theta$ here 25, 20, 15 and 10 degrees). Figure 4.3 shows the number of layers $n_{0}$ (representing eq. 4.5) as

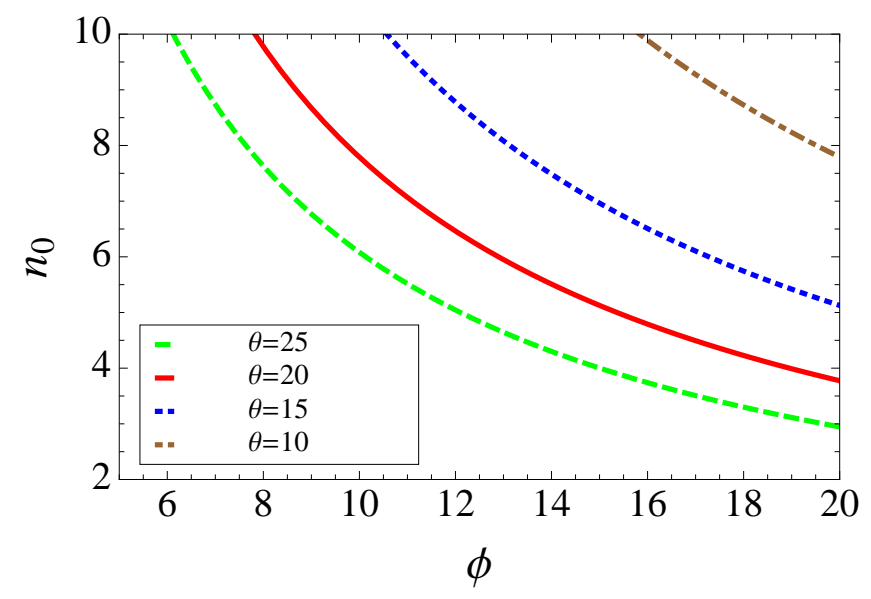

Figure 4.3: Case $L_{0}=0$. Plot of the number of layers $n_{0}$ versus the half angle between the layers $\phi$ (according to eq.4.5) for different divergence half angles $\theta$. When the curves $n_{0}(\phi)$ are above a given fixed $n_{0}$ value, the design of the detector is such that proton energies corresponding to the $n_{0}$ value are detectable. As example for a proton beam with a 40 degrees of divergence $\left(\theta=20^{\circ}\right), 6$ layers can work with a maximum angle $\phi \sim 13^{\circ}$, and for 8 layers $\phi \sim 10^{\circ}$. It is important to note that the proton energy corresponding to the $\mathrm{n}^{\text {th }}$ layer depends on the thickness and composition of the layer. 
a function of $\phi$ for different divergence half angles $\theta$. The relative angle between the layers $(\phi)$ needs to be reduced as much as possible to maximise the possible number of layers maintaining a reasonable dimension of the detector. However, the relative angle $\phi$ should not fall below $10^{\circ}$ in order to have a reasonable spatial resolution of the diagnostic. Indeed, reducing the angle between each layers will reduce the aperture angle of the imaging system, and thus would affect the spatial resolution of the diagnostic in the horizontal axis. As an example, considering a relative angle $\phi=12.5^{\circ}$ between each layer and an imaging system with an angle of view set perpendicular to the proton propagation axis would result in a contraction of a factor of $\approx 5$ in the horizontal axis. Nevertheless, $\phi=12.5^{\circ}$ allows a bigger aperture angle of the imaging system which can look at the layer more from behind (with a smaller angle of view with respect to the proton axis, see section 4.3 .3 for more details about this configuration), resulting in a smaller contraction factor $\approx 1.8$ in the horizontal axis.

\subsection{2 case $L_{0} \neq 0$}

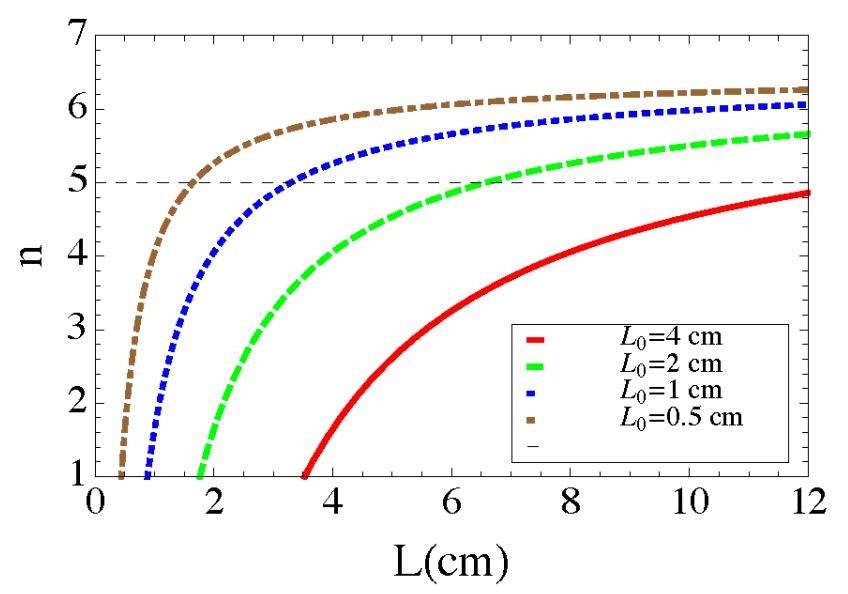

Figure 4.4: The number of scintillator layers $\mathrm{n}$ is represented as a function of the scintillator foil size $(L)$ for different values of $L_{0}$ and for fixed values of $\phi=12^{\circ}$ and $\theta=20^{\circ}$. Different values of $\left(L_{0}=0\right)$ are plotted representing the distance between the detector and the interaction point position. As an example (dashed line in the graph), a detector with $\mathrm{n}=5$ layers needs to be built with a size $L$ greater than: $\sim 1.5$ $\mathrm{cm}\left(L_{0}=0.5 \mathrm{~cm}\right) ; \sim 3.5 \mathrm{~cm}\left(L_{0}=1 \mathrm{~cm}\right), \sim 6.5 \mathrm{~cm}\left(L_{0}=2 \mathrm{~cm}\right) ; \sim 12 \mathrm{~cm}\left(L_{0}=4\right.$ $\mathrm{cm})$.

Assuming a proton divergence with an half angle $\theta=20^{\circ}$ and the relative half angle between 2 layers $\phi=12^{\circ}$, we can represent $n$ (see (4.5)) as a function of $L_{n}$ for different values of $L_{0}$ as shown in fig.4.4. The result is that by increasing $L_{0}$, it increases proportionally $L$ and of course the total longitudinal dimension $L_{n}$. These 
examples show how the energy range of the detector is strongly related to the geometric parameters. Such limitations can be easily overcome with special and dedicated adjustments of the detector design and position in the interaction chamber. In addition other two physical aspects need to be considered: i) from one side, experimental data have shown that proton divergence is reduced by increasing the proton energy ii) from the other side, the contribution of proton multiple scattering in blurring the signal in the latest layers has to be evaluated (see Section 3.4.3). Both effects must be considered in order to properly design the detector.

\subsubsection{Spatial-energy dependence of the proton beam divergence}

We have seen that the detector construction method allows to customize the dimension of each layer, considering the divergence of the proton beam with energy range specific to this layer. However, in the continuation of our study, the detector was built with equal layer dimension, considering the spatial-energy distribution dependence of the proton beam i.e where the divergence is reduced by increasing the proton energy. Consequently, the detector construction turns out to be easier, since each layer has the same dimension. In order to obtain the right design, one condition was to find the "ideal" dimension of the scintillator allowing the beam to fully fit all layers for both low and high energies. One of the solutions is to do Monte Carlo simulations with the expected dimension of the detector for different proton beam divergence and energy.

\subsubsection{Monte Carlo code for numerical simulation (FLUKA)}

FLUKA Monte Carlo Numerical simulations were performed in parallel to experimental studies aiming to predict the stopping range of the proton beam and its transport in solid material. By simulating the propagation of a proton bunch in matter, we were able to determine and customize the composition of our RCF and scintillator detector in order to obtain the adequate proton energy range resolution. Due to the geometry of our scintillator detector, the proton transport through the scintillator layers is subjected to angular scattering. FLUKA was very useful to reproduce the experimental conditions of the experimental campaigns at CMAM and HZDR facilities and thus to be able to study this phenomenon.

FLUKA is an open source multi-purpose Monte-Carlo code simulating the interaction and transport in matter of about 60 different particles such as photons, electrons, hadrons, heavy ions, and electromagnetic particles from few $\mathrm{keV}$ to cosmic ray energy in complete repertory of materials and compounds. It is widely used by the physics community and covers a large range of applications which include, but is not limited to, dosimetry, detector design, target design, radiobiology (including radiotherapy), accelerator shielding where complex geometries and high precision are most of the time involved. FLUKA is jointly developed by the European Organization for Nuclear Research (CERN) and the Italian Institute for Nuclear Physics 
(INFN) [Battistoni et al., 2007, 2016; Fassò et al., 1995; Ferrari et al., 2005] and its development is still in continuous progress, concerning both the user interface and the physical models. The FLUKA input file consists of a text file containing the full description of the beam properties (particle type, energy, distribution, initial divergence,position,direction of the beam,...), the geometry, the material properties, the physics settings (cut-off or threshold for particle production and transport, additional physical effect,...), the requested scoring and executables needed to run a simulation and read the output files. We note that the multiple Coulomb scattering model used in FLUKA for the charged particle transport is an extended approach [Ferrari et al., 1992] based on Molière 's theory [Molière, 1947, 1948, 1955], allowing to work from very small to relatively large steps with a remarkable insensitivity in the results. It is supplemented by an optional single Coulomb scattering method for various optimization requests, with a possibility to switch off completely multiple scattering, when, for example, the limits of Molière 's theory are not satisfied. The charged particles slowing down theory is computed by FLUKA starting from the Bethe-Bloch formalism, introduced previously. Several corrections, extensions and refinements to the standard formulation [Berger et al., 1993b] (completed by [Andersen \& Ziegler, n.d.] at low energy) have been implemented. More details can be found in the literature on www.fluka.org.

\subsubsection{Discussion}

Considering a laser-ion acceleration experiment, the divergence of the particle beam has to be considered in the detector design. Indeed, increasing the total size of the detector will induce an increase of the final beam size, which will entail a reduction of the spatial-energy resolutions. In addition, the last layers will receive a reduced number of protons per unit of area reducing also the detector sensitivity. The optimum detector design must be defined by privileging one parameter with respect to the other, even if a good general rule is to keep a short length detector to preserve a small beam size for a given number of layers. This can be done either by reducing the angle $\phi$ or reducing the lateral size $L$ of the scintillator foils. At this stage of the study, the contribution of multiple scattering is not considered in the design of the detector and will be the subject of future investigations.

\subsection{Characterization of the scintillator detector at the tandem accelerator of the CMAM}

Before using the scintillator detector in an experimental campaign, a characterization was performed at the tandem accelerator from the CMAM of the Universidad Autónoma de Madrid. The response of the BC-400 scintillator have been studied according to different energies and proton fluxes. Indeed, a deep study of its energetic response, in order to estimate the corrective parameters of Birks, is a prerequisite for its correct use for quantitative 2D spatial distribution measurements. The imple- 
mentation of the BC-400 in our first detector prototype has been then tested in the same place, the purpose being to experimentally test the technical feasibility of our detector. We were particularly interested in studying the behavior of a proton beam crossing the detector which can be crucial for the spatial resolution of our diagnostic. This section reports the characterization of the BC- 400 scintillator response and a preliminary study of the detector prototype with a collimated and mono-energetic proton source.

\subsubsection{CMAM facility}

The CMAM provides mono-energetic and mono-directional proton beams with kinetic energies up to $10 \mathrm{MeV}$ with a precision of $4 \mathrm{keV}$. The beams were generated by a Cockcroft-Walton type electrostatic particle accelerator using a target of titanium hydride (TiH2) in the sputtering source [Climent-Font et al., 2004; Pascual, 2004]. This source was chosen instead of the duoplasmatron one, due to the low currents required for the experiment. The proton beam was guided towards the standard multipurpose line equipped with two sets of squared-shaped tantalum slits, separated by two-meter distance from each other, to control the beam size and divergence. In the interaction chamber, with a working pressure of $10^{-6}$ mbar, the point of interaction can be modified using the horizontal rotation axis of $360^{\circ}\left(<0.1^{\circ}\right.$ precision) of a three-axis goniometer, and the vertical axis of $22 \mathrm{~mm}$ range $(0.1 \mathrm{~mm}$ precision) of a translational table. Previous to each irradiation, the proton beam was characterized through a Faraday cup sensitive to currents in the order of picoamperes, placed before the interaction chamber which was also used as beam shutter. During the irradiation, a charge integrator fixed on the sample holder was used to measure the charge that reached the sample.

\subsubsection{Characterization of the BC-400 plastic scintillator response}

One of the major challenge in the application of plastic scintillator-based detectors is the characterization of the energy dependence to particle radiation. It has been established a phenomenon of decrease in the light output (i.e saturation) when charged particles interact with the medium at high stopping power, known as Quenching effect (see Section 3.5.4). Several investigations have been carried out to evaluate the response of equivalent scintillation material BC-400 to protons, deuterons, alpha particles and other heavy ions of various energies [Badhwar et al., 1967; Craun \& Smith, 1970; Ebenau et al., 2016], where experimental data were fitted with Birks model to obtain the quenching parameter $k B$. Therefore, the disparity of these results $(\mathrm{kB}$ mainly between $7 \times 10^{-3}$ and $1.3 \times 10^{-2}$ g.cm ${ }^{-2} \cdot \mathrm{MeV}^{-1}$ ) indicates a need for more accurate measurements in order to understand the processes involved. Indeed, the response behavior of a same scintillator model can be strongly affected by batch to batch differences during manufacturing process, aging effect or energetic secondary electrons (delta rays) created by the heavy primary particle [Meyer \& Murray, 1962; Taylor et al., 1951]. The purpose of this study was to characterize the response de- 


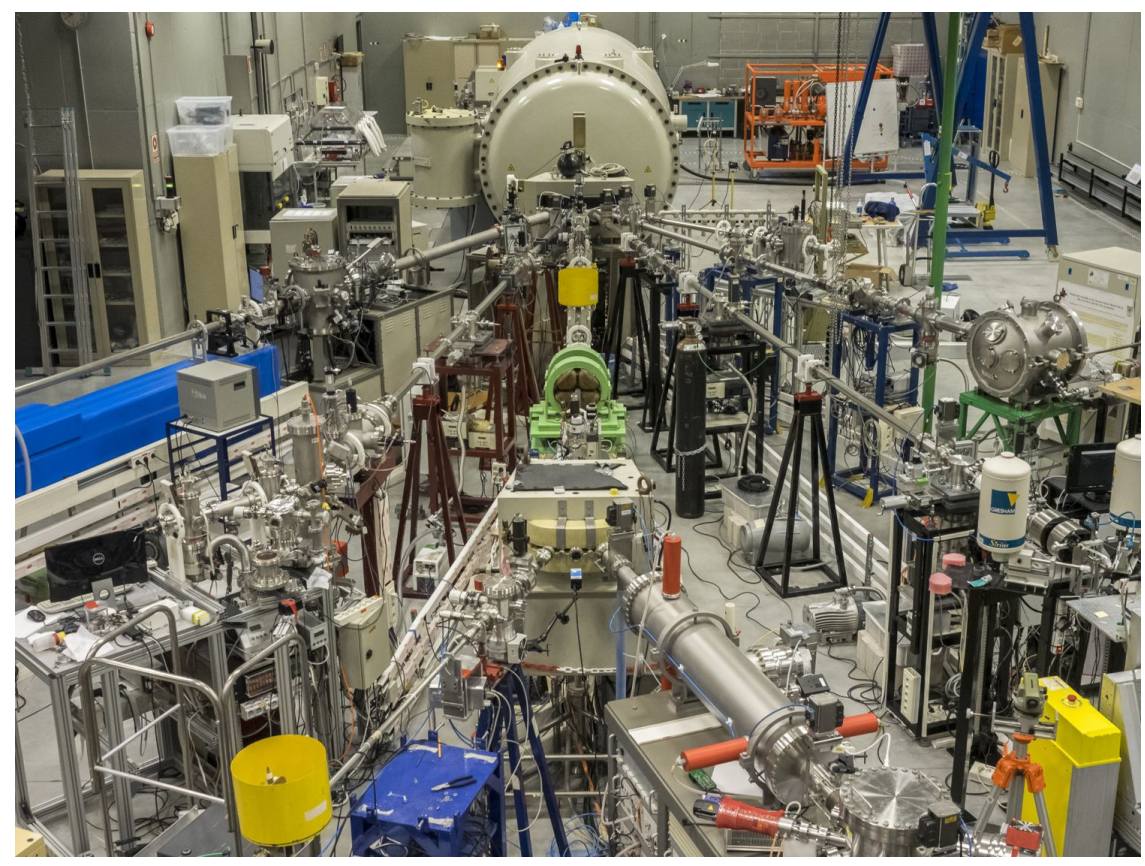

Figure 4.5: CMAM multipurpose beamlines in perspective, with the high energy end of the 5 MV accelerator in the background. From [CMAM, 2021].

pendence to a range of proton fluxes and energies before its implementation into the detector. A small sample from BC-400 model with a thickness estimated of $155 \mu \mathrm{m}$ $\pm 10 \mu \mathrm{m}$ thick with an area of $30 \mathrm{~mm} \times 30 \mathrm{~mm}$ was used for the characterization. It is produced by the Saint Gobain Crystal Company [Saint-Gobain-Crystals, 2020], with a density of $1.032 \mathrm{~g} / \mathrm{cm}^{3}$, a maximum emission wavelength at $423 \mathrm{~nm}$ and a light output of $65 \%$ with respect to the anthracene (see table Figure 4.6 and 4.1 for properties).

Table 4.1: BC-400 scintillator main properties. From [Saint-Gobain-Crystals, 2020].

\begin{tabular}{ll}
\hline \hline Base & Polyvinyl toluene \\
Density & $\rho=1.032 \mathrm{~g} / \mathrm{cm}^{3}$ \\
Refractive index & $\mathrm{n}=1.58$ \\
Softening Point & $70^{\circ} \mathrm{C}$ \\
Light Output (\% of Anthracene) & $65 \%$ \\
Rise time & $0.9 \mathrm{~ns}$ \\
Decay time & $2.4 \mathrm{~ns}$ \\
Wavelength max emission & $423 \mathrm{~nm}$ \\
Bulk light attenuation length & $250 \mathrm{~cm}$ \\
\hline
\end{tabular}

\section{Experimental method}




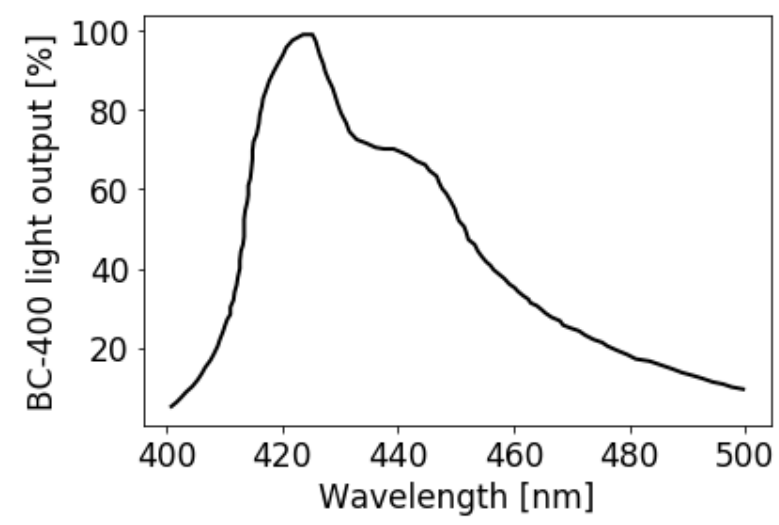

Figure 4.6: Emission Spectra [\%] of the BC-400 scintillator. From [Saint-GobainCrystals, 2020].

The BC-400 scintillator sample was placed in the center of the scattering chamber on the goniometer and was rotated from $15^{\circ}$ respect to the proton beam propagation axis. The scintillation emission was collected from the back side of the scintillator (downstream side) with a CCD camera Point Grey Blackfly monochrome model and an objective NIKON AF-S DX NIKKOR 18-105 mm f/3.5-5.6G ED VR placed outside the chamber at $77.5 \mathrm{~cm}$ from the scintillator. $10 \mu \mathrm{m}$ aluminum filter was placed in front of the scintillator in order to ensure the measurement of the proton current at low kinetic energies, since protons won't reach the end of the sample holder, where they are usually collected for the current measurement. The measurement of the scintillator light output response according to the proton deposited energy was carried out with a set of 12 different proton kinetic energies: $\mathrm{E}_{k, 1}=0.9 \mathrm{MeV} ; \mathrm{E}_{k, 2}$ $=1.1 \mathrm{MeV} ; \mathrm{E}_{k, 3}=1.3 \mathrm{MeV} ; \mathrm{E}_{k, 4}=1.6 \mathrm{MeV} ; \mathrm{E}_{k, 5}=2.5 \mathrm{MeV} ; \mathrm{E}_{k, 6}=3 \mathrm{MeV}$ $; \mathrm{E}_{k, 7}=4 \mathrm{MeV} ; \mathrm{E}_{k, 8}=5 \mathrm{MeV} ; \mathrm{E}_{k, 9}=6 \mathrm{MeV} ; \mathrm{E}_{k, 10}=7 \mathrm{MeV} ; \mathrm{E}_{k .11}=8 \mathrm{MeV}$ $; \mathrm{E}_{k .12}=10 \mathrm{MeV}$. During this measurement, the proton flux could vary slightly between each energy shot (between 0.7 and $0.85 \mathrm{nA}$ corresponding to $4.4 \times 10^{9}$ to 5.3 $\times 10^{9}$ proton $/ \mathrm{s}$ ) but was taken into account in the analysis since scintillator light output was measured in number of photons generated per incident proton unity. The measurement of the scintillator response according to the proton flux was performed with 2 different proton kinetic energies of $10 \mathrm{MeV}$ and $3 \mathrm{MeV}$, with a proton flux varying for each between $\approx 1 \times 10^{10}$ up to $\approx 7 \times 10^{10}$ protons.cm ${ }^{-2} \cdot \mathrm{s}^{-1}$, corresponding to a given current from 0.25 to $1.95 \mathrm{nA}$. For both measurements, recording exposure time and amplification gain of the CCD could be modified according to the signal resolution. The proton beam shape was controlled by a tantalum collimator to get a lozenge-like shape beam of about $7 \mathrm{~mm} \times 7 \mathrm{~mm}$.

\section{CCD count to photons number conversion}

In order to obtain a precise measurement of the quenching parameter $k B$, an accurate estimation of the number of photons emitted by our scintillator during the interaction 
with the proton beam was needed. The brightness informations of each pixel of the CCD output image are digital counts expressed as grey values. In order to be able to recalculate the number of corresponding detected photons, several parameters must be taken into account during the different phases of conversion from photon to digital counts. Figure 4.7 illustrates the most important steps in digitizing the light in a

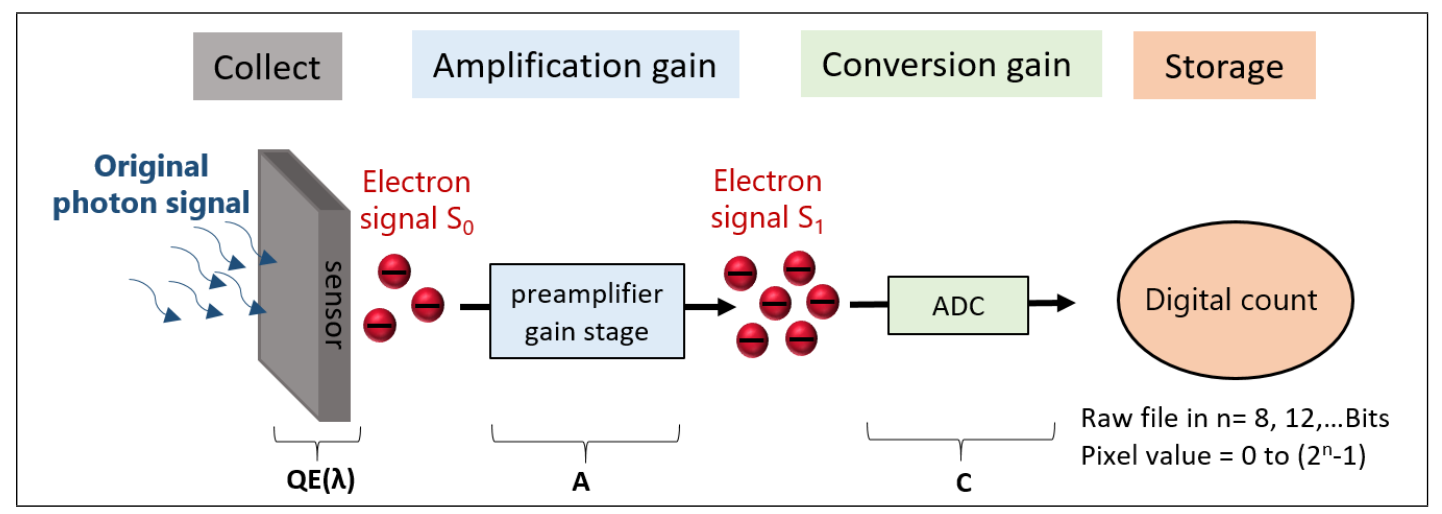

Figure 4.7: Main step conversion of the CCD signal.

single pixel:

- The collect of the photons and their conversion into electrons.

When the incoming photons arrive at the sensor area, only a few part of the collected photons will be converted into electrons. The ratio of electron generated during this process is called Quantum Efficiency and is wavelength dependent with a scaling factor $\mathrm{QE}(\lambda)$ :

$$
Q E(\lambda)=\frac{\text { generated electrons }}{\text { collected photons }} .
$$

Figure 4.8 represents the quantum efficiency curve $\mathrm{QE}(\lambda)$ in percent and shows a variation between $60 \%$ and $85 \%$ within this range of the BC-400 scintillator emission $(400 \mathrm{~nm}-500 \mathrm{~nm})$. In our case, a rough estimation of the converted incident photons into electrons was estimated by evaluating the average quantum efficiency $\langle Q E\rangle$ of about $75 \%$ for the BC-400 spectra with the following method:

$$
\langle Q E\rangle=\frac{\int_{\lambda=400 \mathrm{~nm}}^{\lambda=500 \mathrm{~nm}} Q E(\lambda) L(\lambda) d \lambda}{\int_{\lambda=400 \mathrm{~nm}}^{\lambda=500 \mathrm{~nm}} L(\lambda) d \lambda}
$$

with $Q E(\lambda)$ and $L(\lambda)$ representing the Quantum efficiency of the $\mathrm{CCD}$ and relative light output of the BC-400.

- The amplification of the electron signal in the preamplifier gain stage. In some cases, the electrons signal can be then multiplied by a factor A before it is being 


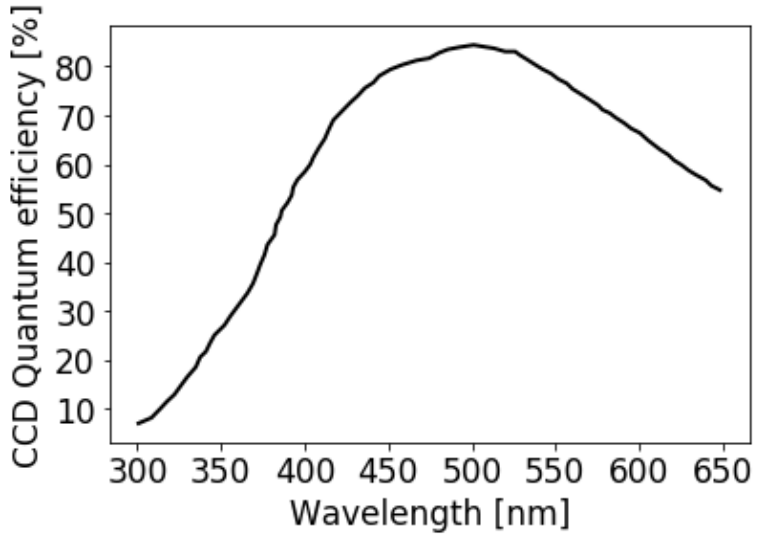

Figure 4.8: Spectral domain of the quantum efficiency [\%] for the CCD camera Point Grey Blackfly monochrome model BFLY-PGE-23S6M-C.

transformed into a digital signal. This process occurs in the preamplifier gain stage and the multiplication factor is chosen by the user in the setting of the CCD software. The gain is expressed in decibel $(\mathrm{dB})$ and measures the ability to increase the amplitude of a signal from the input to the output port. It reads

$$
\text { Gain }[\mathrm{dB}]=20 \log A \quad \text { with } A=\frac{\text { output signal }}{\text { input signal }}
$$

The covered gain range of our CCD model is going up to $30 \mathrm{~dB}$, corresponding to a maximum factor $\mathrm{A} \approx 31.6$.

- Conversion of the analogue signal into a digital unit.

Finally, the electrons leaving the preamplifier gain stage are then digitized at the Analogue-to-digital converter (ADC). Each pixels are thus expressed in nbits Analogue-to-Digital Unit (ADU), represented by "counts", expressed in grey scale with $2^{n}-1$ possible values. Each count represents an exact number of electrons. The ratio between the analogue signal value to digital grey scale value is referred to as a conversion gain and is measured in electrons per ADU ( $\left.\mathrm{e}^{-} / \mathrm{ADU}\right)$. In our case, a 16-bits format was used to record the signal, thus leading to a conversion gain of $\mathrm{C}=0.52 \mathrm{e}^{-} / \mathrm{ADU}$.

Therefore, in order to back calculate to the original signal in electrons, the knowledge of the bias offset is required. It is an artificially induced electronic offset which ensures that the Analogue-to-Digital Converter (ADC) always receives a positive signal value. In order to get this value, one must take a dark reference image, calculate the average intensity in Grey scale values of all the pixels and subtract this offset from each pixel in the sample image. Our CCD camera was coupled with an objective NIKON AF-S DX NIKKOR 18-105 $\mathrm{mm} \mathrm{f/3.5-5.6G} \mathrm{ED} \mathrm{VR.} \mathrm{The} \mathrm{transmittance} \mathrm{T}$ of the whole optics system composing our NIKON objective must be taken into account before getting 
the original number of photon reaching it. We could roughly estimate the average transmittance through such group of optics system for the BC-400 spectra of about $\langle T\rangle=83 \%^{1}$. The number of photon for each pixel can be thus calculated by the following formula:

$$
\text { Photon number }=\frac{(\text { Pixel value }- \text { bias offset }) \times C}{A \times\langle Q E\rangle \times\langle T\rangle} .
$$

Taking into consideration that the number of photon obtained will correspond to the number of photon detected in a given exposure time set in CCD parameter.

\section{Energy dependence}

Figure 4.10 represents the response of the BC-400 scintillator sample according to the total energy loss in the layer $\Delta E$ by proton with initial kinetic energies from $E_{k, 1}$ to $E_{k, 12}$. The light output $L$ is expressed in photon ( $\mathrm{Ph}$.) per unit of proton, considering the detection solid angle of the CCD. The total energy loss $\Delta E$ per proton was obtained by integrating the proton Bragg curve extracted from FLUKA simulations (see Figure 4.9). We can notice two tendencies of curves, directly related to the phenomenon of quenching. Indeed, by referring to Figure 4.9, one can observe that protons of kinetic energies between 0.9 and $3 \mathrm{MeV}$ (black cross on Fig. 4.10) will be totally stopped inside our sample BC-400 of $155 \mu \mathrm{m}$, while the protons of higher energy from 4 up to $10 \mathrm{MeV}$ (black circles on Fig. 4.10) will deposit only a part of their energy all along the sample and continue their way. According to the Birks equation (3.89), one can see that the quenching effect depends directly on the energy deposited per unit length and will be accentuated for lower kinetic energies, for which the stopping power of the material becomes significantly high. Thus, an amount of energy deposited on a short path around the Bragg peak will generate a lower scintillation response than the same amount deposited on a longer path, outside the Bragg peak. An example of these two cases is observed in Figure 4.10 (dashed-lines rectangles) where the energy deposited by a proton of $1.6 \mathrm{MeV}$ and $6 \mathrm{MeV}$ inside our sample is similar (see Table 4.2) but does not generate the same response from the scintillator.

Finally, the behavior of the black cross data can be explained by a quenching with a strong effect already established in the first steps of the proton propagation into the scintillator due to the high stopping power of the material around the Bragg peak, thus damaging the medium and attenuating considerably the scintillator response. In the second case (black circles), the scintillator response seems to increase nonlinearly with $\Delta E$ (i.e increase with lower kinetic energies) up to a saturation regime. According to figure 4.9, proton kinetic energies close to $10 \mathrm{MeV}$ undergo a fairly low and constant stopping power throughout their propagation in the sample (which may already lead to a slight extinction in the first steps of their propagation) while energies close to $4 \mathrm{MeV}$ start experiencing higher stopping power in their later stages

\footnotetext{
${ }^{1}$ We find difficulties to get the exact spectral transmittance of the NIKON AF-S DX NIKKOR 18-105 mm f/3.5-5.6G ED VR in the literature. However, we could find a similar objective model, with comparable construction and composition of optics.
} 


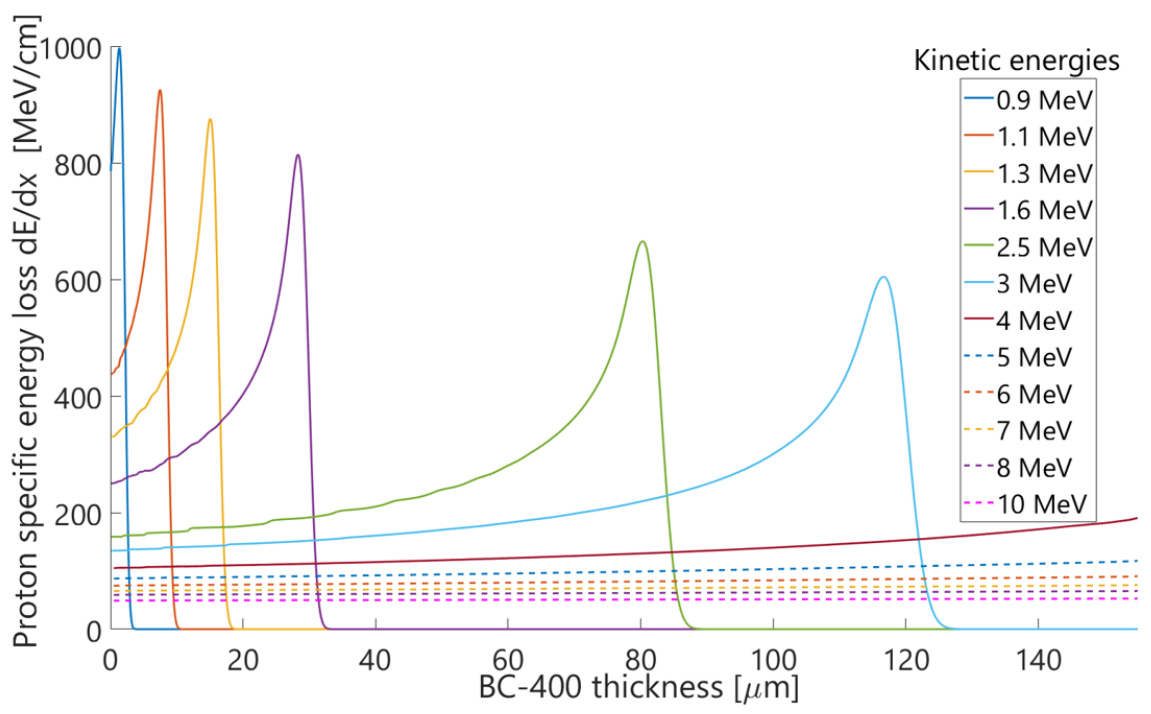

Figure 4.9: FLUKA simulation for 0.9 up to $10 \mathrm{MeV}$ proton kinetic energies showing the range and the theoretical Bragg curve shape inside $155 \mu \mathrm{m}$ thick BC-400 scintillator.

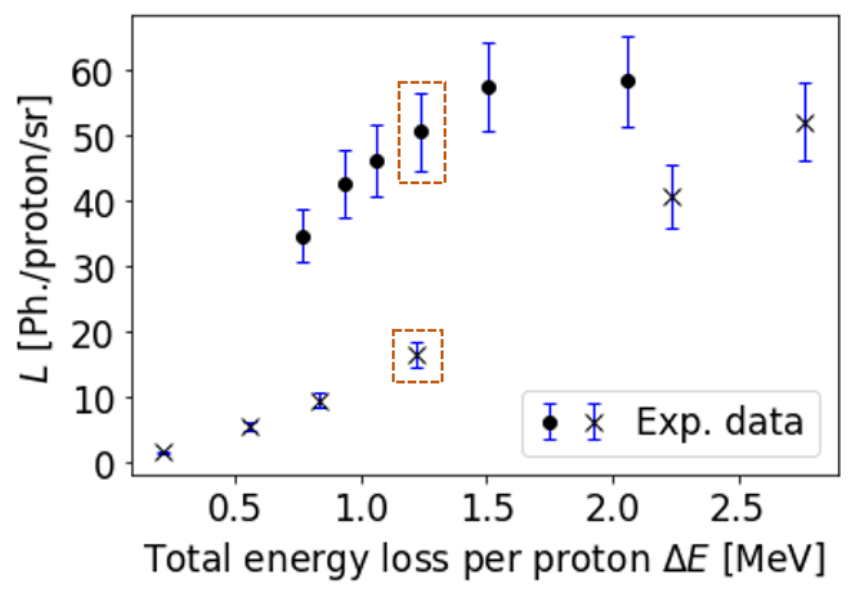

Figure 4.10: Light output response of $155 \mu \mathrm{m}$ thick BC-400 scintillator according to proton energy loss. Black crosses correspond to cases where energies are deposited by proton totally stopped inside the sample. Black circles correspond to cases where proton deposit their energy all along the sample without being totally stopped by the sample. Dashed-lines rectangles highlight both cases for similar deposited energy.

of propagation through sample BC-400, resulting in much higher stopping power and greater attenuation of the luminescence in the last path.

\section{Interpretations using the Birks model}




\begin{tabular}{c|ccccccccccccc}
\hline \hline $\begin{array}{c}\text { Proton initial } \\
\text { kinetic energy [MeV] }\end{array}$ & 0.9 & 1.1 & 1.3 & 1.6 & 2.5 & 3 & 4 & 5 & 6 & 7 & 8 & 10 \\
\hline $\begin{array}{c}\text { Total energy loss } \Delta E \\
\text { in } 155 \mu \mathrm{mBC}-400^{*} \\
{[\mathrm{MeV}]}\end{array}$ & 0.22 & 0.56 & 0.84 & 1.22 & 2.23 & 2.76 & 2.06 & 1.51 & 1.24 & 1.06 & 0.94 & 0.767 \\
\hline
\end{tabular}

* Simulations include $10 \mu \mathrm{m}$ aluminum placed in the front side of the scintillator

Table 4.2: Energy loss by different proton kinetic energies in a $155 \mu \mathrm{m}$ thick BC-400 plastic scintillator obtained by Monte Carlo simulation FLUKA.

In order to be able to adjust the characteristic parameters of luminescence of our scintillator, we made a parametric adjustment of the Birks law on our experimental data measured previously. The detected light output $L$ generated by the interaction of a proton of kinetic energy $E_{k}$ depositing its energy in the scintillator of thickness $\Delta z$ is then obtained by integrating equation 3.89 such as

$$
L[\mathrm{Ph} . / \text { proton } / \mathrm{sr}]=\int_{z=0}^{z=\Delta z} \frac{d L}{d z} d z=\int_{z=0}^{z=\Delta z} \frac{A d E / d z}{1+k B d E / d z} d z,
$$

we remind that $\mathrm{A}$ is the scintillation efficiency $\left[\mathrm{Ph}_{\mathrm{MeV}} \mathrm{MeV}^{-1} \mathrm{sr}\right], \mathrm{kB}$ the quenching parameter $\left[\mathrm{cm} \cdot \mathrm{MeV}^{-1}\right]$ (usually expressed in $\left[\mathrm{g} \cdot \mathrm{cm}^{-2} \cdot \mathrm{MeV}^{-1}\right]$ ) and $\mathrm{dE} / \mathrm{dz}$ the proton Bragg peak curve $\left[\mathrm{MeV} \cdot \mathrm{cm}^{-1}\right]$. The proton Bragg peak curve dE/dz for each proton of kinetic energy $E_{k, 1} \ldots E_{k, 12}$ was extracted from FLUKA simulations. In each iteration, the program calculates the total luminescence value for each proton Bragg curve, obtained with a set of parameters $\mathrm{A}$ and $\mathrm{kB}$. The parameters are then adjusted to minimize the difference between the simulated and experimental values in the next iteration. We estimated $\mathrm{A}=85 \mathrm{Ph} \cdot \mathrm{MeV}^{-1}$.sr and $\mathrm{kB}=1.4 \times 10^{-2} \mathrm{~cm} \cdot \mathrm{MeV}^{-1}$ which is $\approx 1.4 \times 10^{-2}$ g.cm ${ }^{-2} \cdot \mathrm{MeV}^{-1}$, with the coefficient of determination $\mathrm{R}^{2}=0.99$. The fitting of experimental data by Birks Law is represented in Figure 4.11. The adjustment parameter find for $\mathrm{kB}$ is in good agreement with the literature of [Craun \& Smith, 1970] $\left(\mathrm{kB}=1.31 \times 10^{-2}\right.$ g.cm $\left.{ }^{-2} \cdot \mathrm{MeV}^{-1}\right)$, [Gooding \& Pugh, 1960] $(\mathrm{kB}=1.32$ $\times 10^{-2}$ g.cm $\left.{ }^{-2} \cdot \mathrm{MeV}^{-1}\right)$ or [Badhwar et al., 1967] $\left(\mathrm{kB}=1.26 \times 10^{-2} \mathrm{~g} \cdot \mathrm{cm}^{-2} \cdot \mathrm{MeV}^{-1}\right)$. Knowing the adjusted parameters for our sample BC-400, it is possible to insert them in the original Birks equation (3.89) in order to understand the response of the scintillator $d L / d z$ according to the specific energy loss $d E / d z$. Figure 4.12 shows that a significant saturation due to quenching effect occurs at stopping powers higher than $30 \mathrm{MeV} / \mathrm{cm}$.

\section{Proton flux dependence}

In order to confirm the linearity response to fluxes, the scintillator sample has been exposed to different ranges of proton fluxes and proton kinetic energies of 3 and 10 $\mathrm{MeV}$. The response curves are shown in Figure 4.13. Scintillator BC-400 light output was found to be proportional to proton flux up to about $10^{11}$ protons.cm ${ }^{-2} \cdot \mathrm{s}^{-1}$ and 


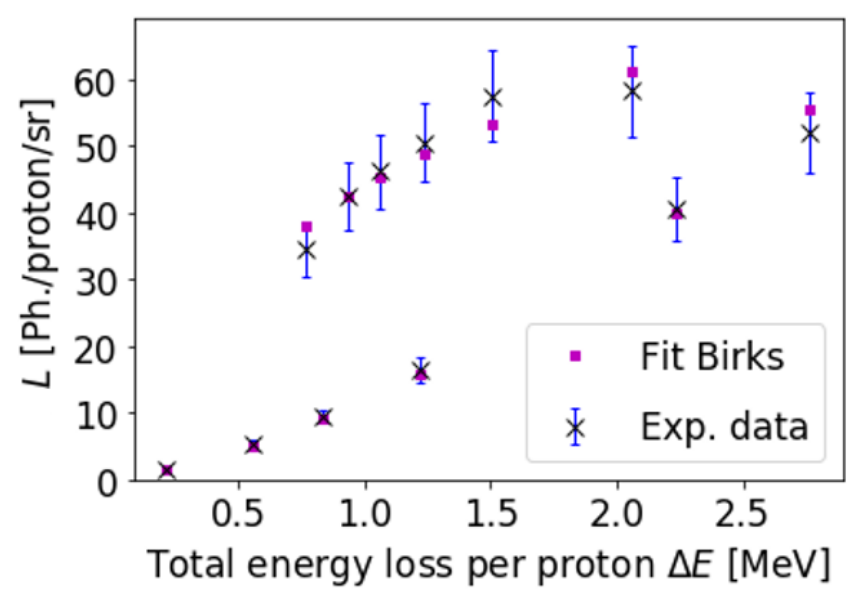

Figure 4.11: Black crosses: Light output response of $155 \mu \mathrm{m}$ thick BC-400 scintillator according to proton energy loss. Magenta squares: Fitting of the experimental data with Birks equation (4.10).

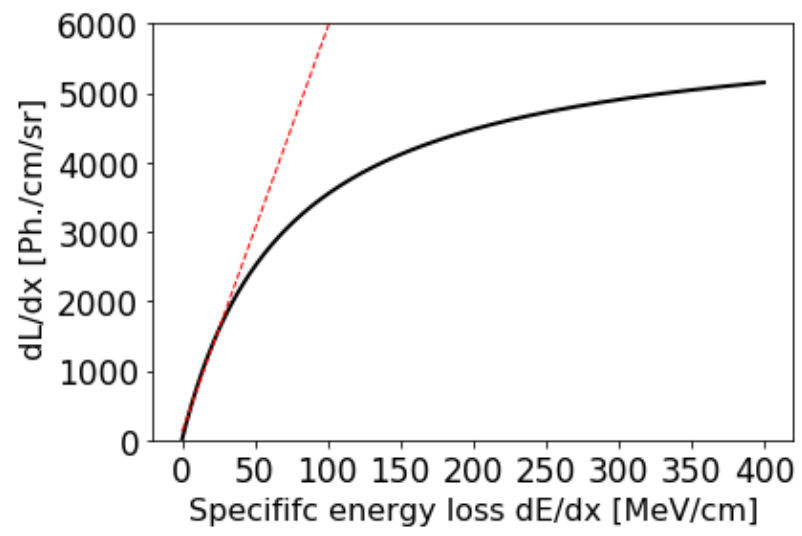

Figure 4.12: BC-400 scintillator response as a function of proton beam flux for 3 and $10 \mathrm{MeV}$ kinetic energy.

are consistent with those obtained by [Torrisi, 2000]. It is important to note that even under different degree of saturation due to quenching effect, the scintillator response stays linear to different proton fluxes.

\section{Discussion}

The response of a BC-400 scintillator has been characterized by several energies and proton fluxes, at the CMAM facility. A linear response for proton flux up to about $10^{11}$ protons. $\mathrm{cm}^{-2} \cdot \mathrm{s}^{-1}$ has been observed. Conversely, it shows a strong attenuation of the response to deposited energy at high stopping power due to quenching effect. Indeed, the quenching effect has been observed at low proton energy (high 


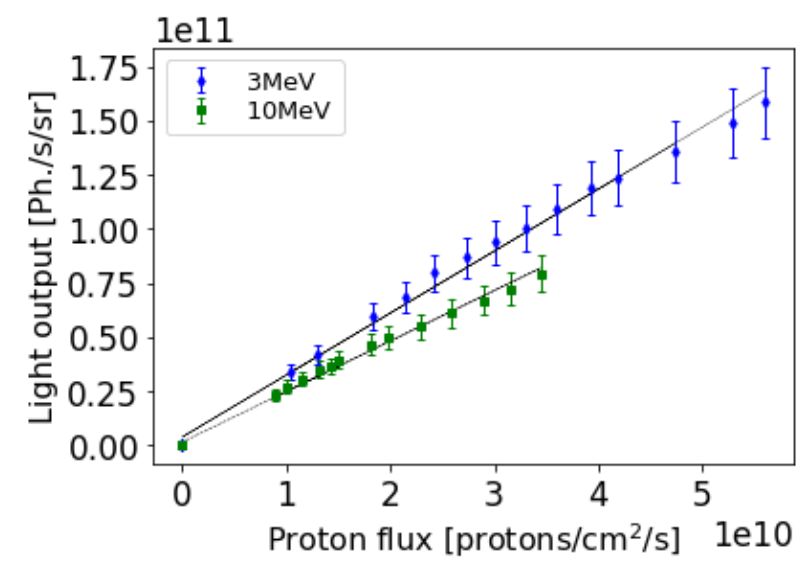

Figure 4.13: BC-400 scintillator response as a function of proton flux for 3 and 10 $\mathrm{MeV}$.

stopping power), as predicted, and appears for specific deposited energy higher than $30 \mathrm{MeV} / \mathrm{cm}$. However, it could be suitable for quantitative 2D spatial distribution measurements considering correcting factor to rectify the quenching effect. While the initial characterization have been successful, care must be taken when applying the above scaling parameters to experimental results. This work marks the first steps to follow for a total calibration of a $2 \mathrm{D}$ scintillator based proton detector but the characterization may be improved by extending the proton source energy range, in order to explore a response outside the quenching regime. Additional work may also be undertaken in order to characterize deeply the usable dynamic range of the scintillator for its operation under extreme conditions of laser-plasma interaction. Damage threshold and lifetime of plastic scintillators must be understood since the luminescence may reduce depending of their exposure time to ionizing radiations [Torrisi, 1997]. The choice of product brand should be reconsidered since uncontrollable variations of the scintillator thickness up to $30 \%$ have been observed during the fabrication process by the Saint Gobain Crystal Company [Saint-Gobain-Crystals, 2020], thus generating uncertainties in the interpretation of the results if the thickness over the whole scintillator area is not well characterized by the user.

\subsubsection{Implementation and preliminary study of the detector with a collimated and mono-energetic proton beam}

This experimental campaign at CMAM allowed to test the general functionality of the detector and the signal detection when irradiated by a proton beam. The detector was composed by 10 scintillator layers, BC-400 model (see table 4.1), placed one after each other with an angle of $25^{\circ}$ between them. Each layer has a stated thickness of $150 \mu \mathrm{m}$ according to the manufacturer with a free detector area of $20 \mathrm{~mm}$ $\times 20 \mathrm{~mm}$. However, due to uncontrollable variation during the fabrication process 
of the company, each layer may have a variation of $30 \%$ from the stated thickness. Estimation of the actual thickness was done with a high precision caliper and is presented in Table 4.3. $10 \mu \mathrm{m}$ thick aluminum foil was placed on the front side of each layer to protect it from the previous scintillator layer light emission. Fig.4.14 shows a customized version of the detector with all the relevant parameters. Due to the very low beam divergence of the proton beam generated in conventional accelerator, there were no constraints related to the possible number of layers and their dimension.

\section{Experimental method}

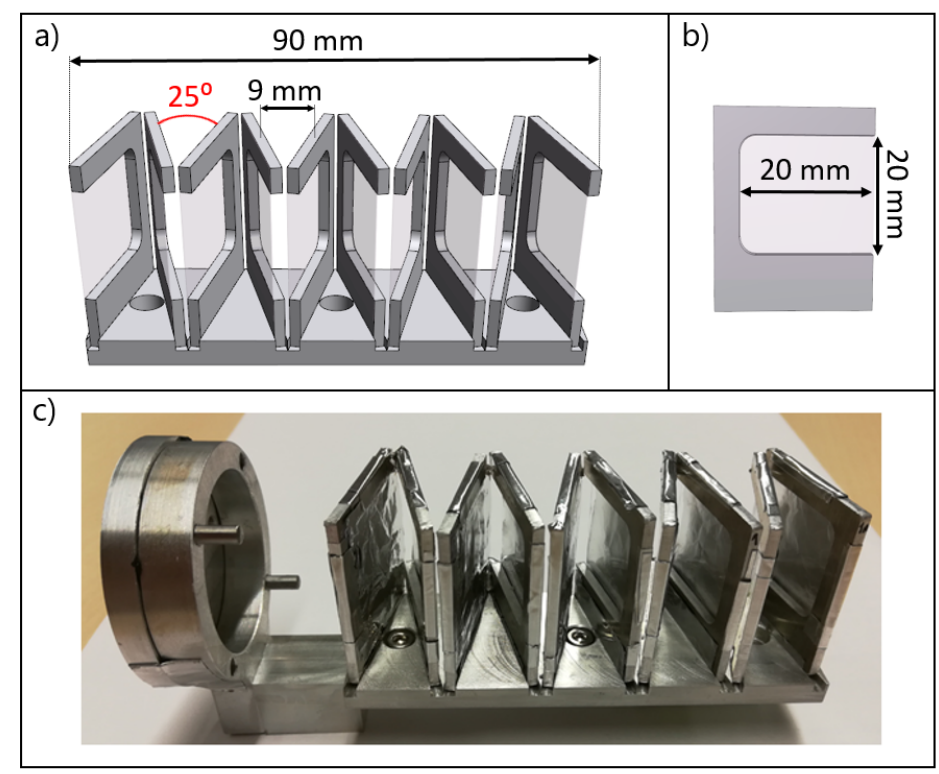

Figure 4.14: a) 3D draw of the detector prototype with a longitudinal dimension of the base between the first and the last layer of $90 \mathrm{~mm}$. Each layer is separated from each other with an angle of $25^{\circ}$. b) 3D draw of the individual holder front view and its scintillator layer with a dimension of $20 \times 20 \mathrm{~mm}$. c) Picture of the detector lateral view (the circular piece on the left is used to fix the detector to the goniometer).

The detector was placed in the middle of the interaction chamber, on the front part of a 4 -axis goniometer, able to rotate $360^{\circ}$ around the propagation axis of the proton beam. The emission was collected from the rear side of each scintillator (downstream side) with a CCD camera Point Grey Blackfly monochrome model $(1920 \times 1200$ pixels) and an objective NIKON AF-S DX NIKKOR 18-105 mm f/3.5-5.6G ED VR placed outside the chamber at about $83.5 \mathrm{~cm} \pm 0.5 \mathrm{~cm}$ from the first layer and $81 \mathrm{~cm}$ $\pm 0.5 \mathrm{~cm}$ from the tenth layer (see figure 4.15 ). The amplification gain was fixed to $25 \mathrm{~dB}$ and the recording exposure time was set at $10 \mathrm{~ms}$. The original design of the detector is using two cameras looking to odd and even layers from opposite sides but 

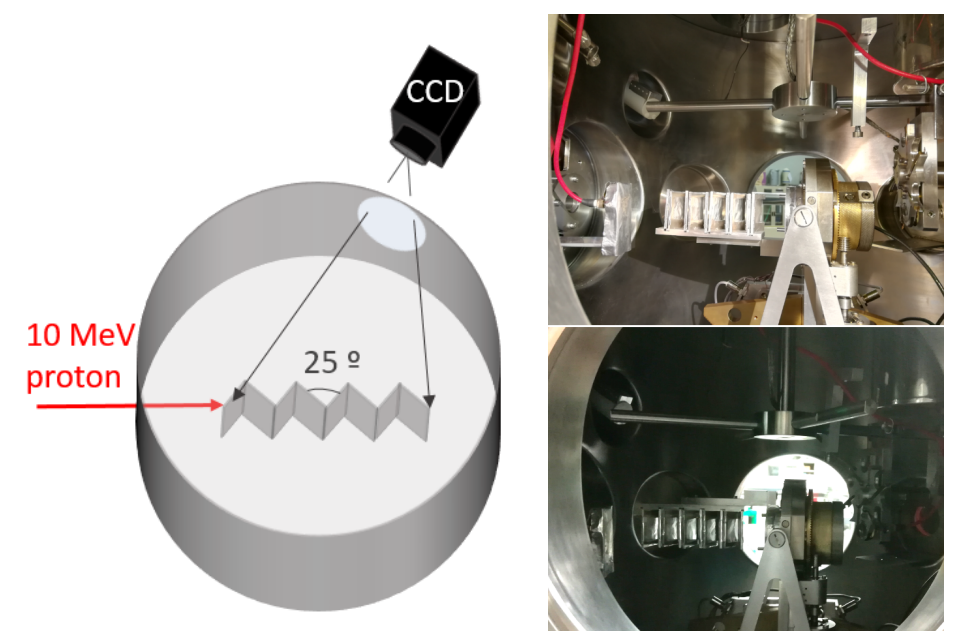

Figure 4.15: (Left) Top view of the interaction chamber with the detector place inside and the camera set outside the chamber for recording the signal. (Right) The top picture represents the configuration 1 , where the odd number scintillator layers are imaged by the camera. The bottom picture represents the configuration 2 with the imaging of the even scintillator layers.

due to the physical constraints of the interaction chamber and considering the proton source very stable, two configurations of irradiation were done to be able to image the full detector with the same camera. The odd layers with the numbers 1,3,5,7 and 9 were pictured when the goniometer was in normal position (rotation axis at $0^{\circ}$ ) and the even layer numbers $2,4,6,8$ and 10 when the goniometer was at $180^{\circ}$ of rotation (see figure 4.15). The proton beam energy was fixed to $10 \mathrm{MeV}$ and interacts at the central zone of the scintillator. The initial beam geometry was measured by using a small piece of glass scintillator (CMAM's property) imaged from a small window on the top size of the chamber and was found to be a quadrilateral geometry of $\approx 3.5(y)$ $\times 4.5(x) \mathrm{mm}$. The collimated proton beam current was slightly varying during the two configuration measurements from 6.07 to $6.47 \mathrm{nA}$, corresponding to a proton flux of $3.76-4.04 \times 10^{9} / \mathrm{s}$. However, this variation was considered in the reconstruction of the scintillator light output signal.

\section{Detector response}

The signal detected by the CCD is represented in Figure 4.16 with the pixel intensity values expressed in Grey scale. It shows a clear signal of scintillation emission up to the $6^{\text {th }}$ layer with a very weak signal observed on the $7^{\text {th }}$, which can be explain by the Bragg peak tail interacting with this layer. This result was confirmed by simulating the total energy loss $\Delta E$ of a $10 \mathrm{MeV}$ proton beam into the detector using the FLUKA modeling code. Despite the fact that the proton beam was aligned with the center of the detector layers, one can observe a slight lateral shift of the beam on the 


\begin{tabular}{cccccccccccc}
\hline \hline & \multicolumn{1}{c}{ Composition of the scintillator detector } & & & \\
\hline Layer & 1 & 2 & 3 & 4 & 5 & 6 & 7 & 8 & 9 & 10 \\
Thickness $[\mu \mathrm{m}]$ & 120 & 180 & 140 & 160 & 190 & 130 & 150 & 170 & 140 & 130 \\
\hline
\end{tabular}

Table 4.3: Composition of the scintillator detector. We note that each scintillator layer was preceded by $10 \mu \mathrm{m}$ aluminium filter. Due to uncontrollable variation during the fabrication process of the company, each scintillator $(\mathrm{S})$ has a different thickness and was placed in random order when building the detector. The scintillator thickness was estimated with a high precision caliper: $\mathrm{S}_{1}=120 \mu \mathrm{m} ; \mathrm{S}_{2}=180 \mu \mathrm{m} ; \mathrm{S}_{3}=140$ $\mu \mathrm{m} ; \mathrm{S}_{4}=160 \mu \mathrm{m} ; \mathrm{S}_{5}=190 \mu \mathrm{m} ; \mathrm{S}_{6}=130 \mu \mathrm{m} ; \mathrm{S}_{7}=150 \mu \mathrm{m}$ with an incertitude of \pm $10 \mu \mathrm{m}$.

odd layers due to a small offset of the odd holder layers. The signal in the even layers $(2,4,6)$ seems slightly higher due to the current variation for the two measurements. The spatial resolution of the imaging system was evaluated to $60 \mu \mathrm{m}$ in the vertical axis with a contraction factor in the horizontal axis varying from 1.79 for the $1^{\text {st }}$ layer up to 1.92 for the $6^{\text {th }}$ layer, corresponding to a spatial resolution of $107 \mu \mathrm{m}$ up to $115 \mu \mathrm{m}$ respectively. An increase of the signal area is observed as the beam
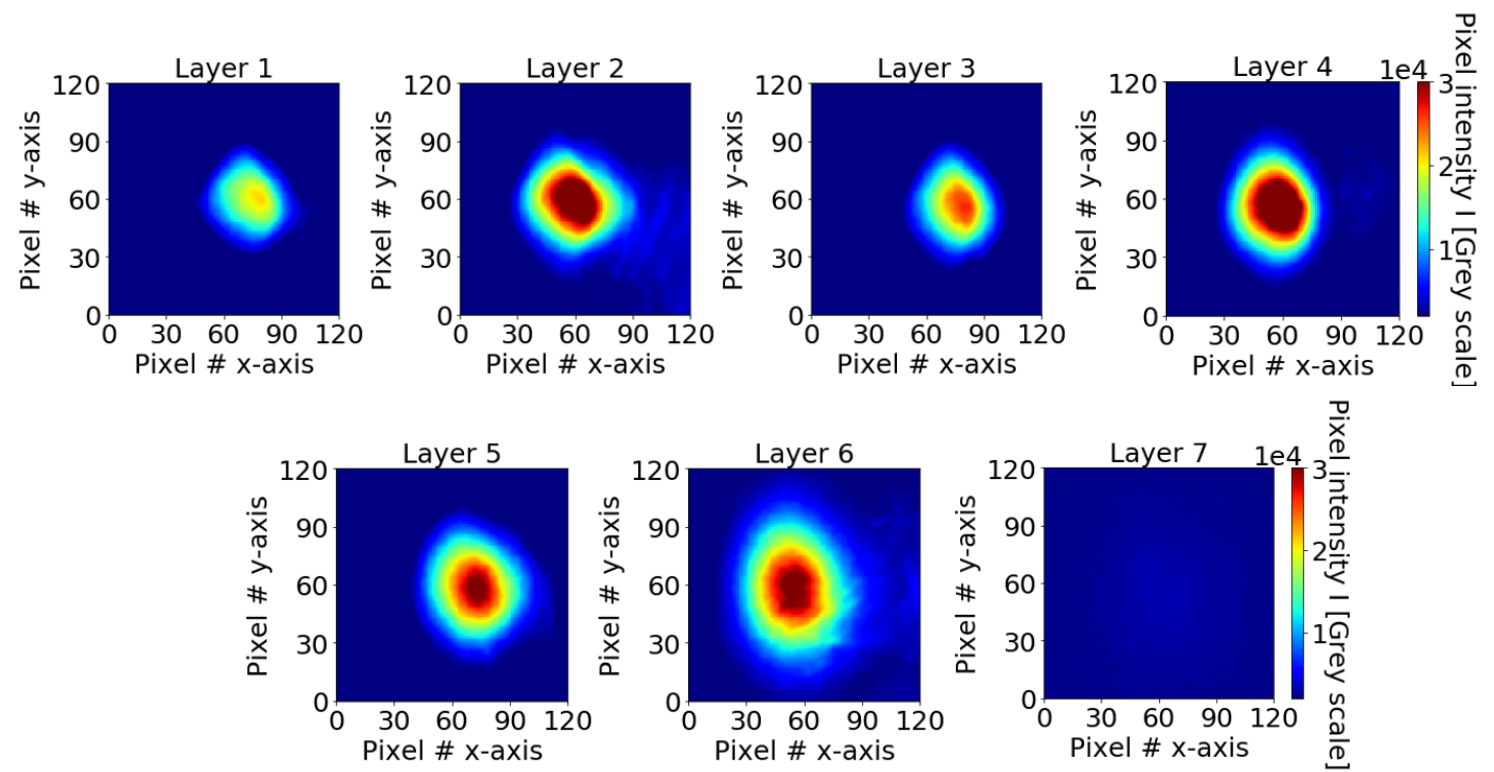

Figure 4.16: 2-D pixel intensity signal detected with a CCD camera Point Grey Blackfly monochrome model $(1920 \times 1200$ pixels $)$ and an objective NIKON AF-S DX NIKKOR 18-105 mm f/3.5-5.6G ED VR, in Grey scale for the first seven scintillator layers irradiated by a $10 \mathrm{MeV}$ proton beam. The surface is measured at $13.5 \%$ of the maximum value (for better visualisation, a Gaussian filter was applied to smooth the signal with a standard deviation $\sigma=1$ ). 
propagates through the layers due to angular scattering. Figure 4.17 estimates the lateral scatter between layer 1 and 6 for experimental and simulated data (FLUKA). The measurement shows quite similar result with an increase of about $2.3 \mathrm{~mm}\left(2.9^{\circ}\right.$ full angle) at the $6^{\text {th }}$ layer for the experimental data and about $2 \mathrm{~mm}\left(2.6^{\circ}\right.$ full angle $)$ for the simulated one. Figure 4.18(a) shows the signal recorded during the two configurations of irradiation and have been combined to give the signal in a single image. As example the total deposited energy per proton for each scintillator layer has been extracted from FLUKA simulation and it is represented in the figure 4.18(b). It is in good agreement with the scintillator response in figure 4.18(c). The light output has been obtained from recorded pictures by the conversion of pixel value into photons. We can observe a slight flattening of the scintillator response around the Bragg peak (high stopping power) that can be interpreted as a saturation of the response due to the quenching effect.

\section{Discussion}

Over the next few years, the need for development of charged particle detectors that are capable of operating on HRR for laser facilities while maintaining many of the characteristics of conventional passive diagnostics will increase significantly. A first prototype of scintillator-based 2D proton detector has been preliminary tested at the tandem accelerator of the CMAM facility and has shown promising results for replacement of the classical $\mathrm{RCF}$ and represents a new class of online detectors to support laserplasma physics experiments in the emerging high power laser systems operating at HRR. However, while a system like this could be used to monitor overall beam shape, the possibility of using this detector for any fine structure that may be of interest such as proton radiography is still a challenge, due to the issue of lateral scattering along the stack. 


\section{Experimental data}

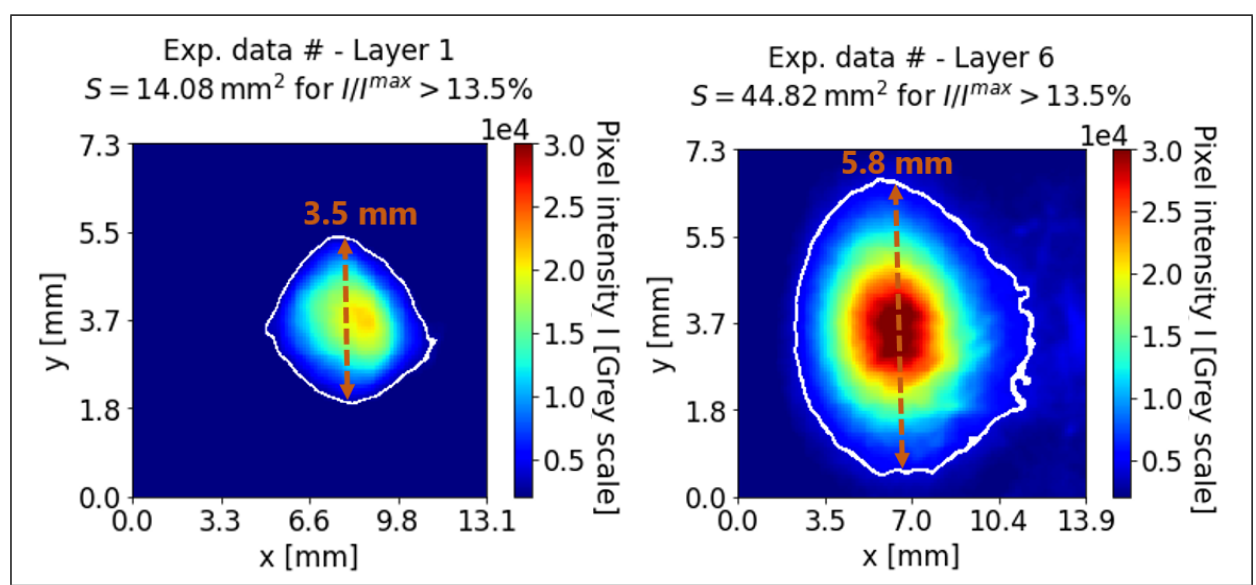

\section{Monte Carlo \\ simulation (FLUKA)}

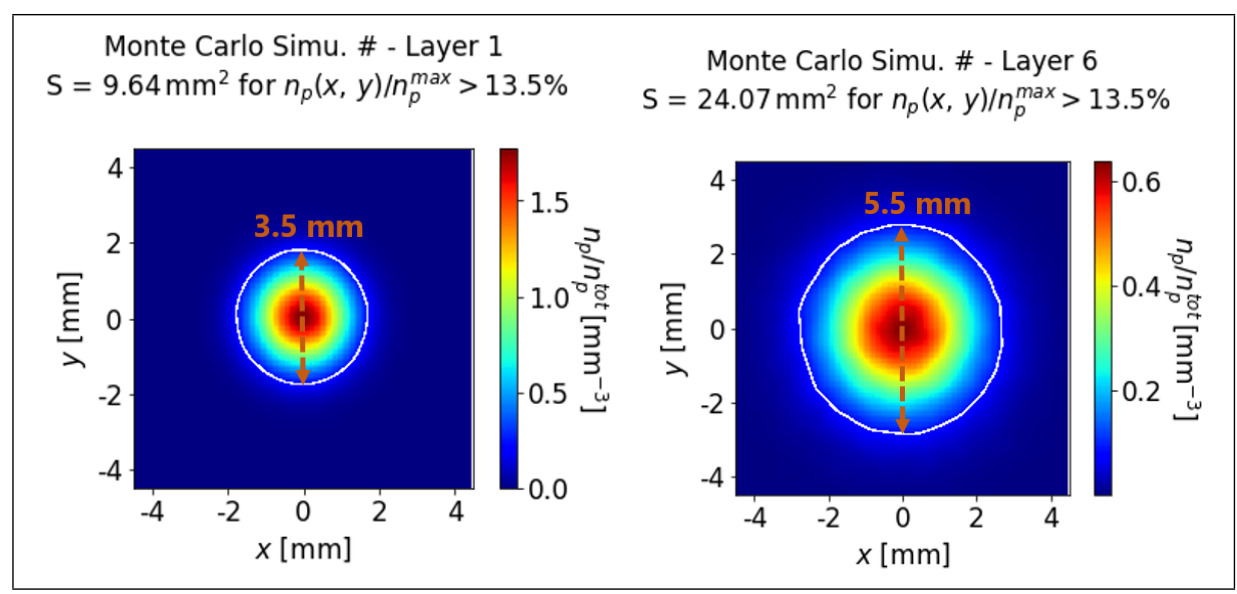

Figure 4.17: (Top) Experimental data: 2-D pixel intensity signal detected by the CCD in Grey scale. The surface is measured at $13.5 \%$ of the maximum value. The spatial resolution of the imaging system is about $60 \mu \mathrm{m}$ in vertical and $107 \mu \mathrm{m}$ $(115 \mu \mathrm{m})$ for the $1^{\text {st }}\left(6^{\text {th }}\right)$ layer. The lateral scattering is estimated about $2,3 \mathrm{~mm}$. (Bottom) FLUKA simulation: Normalized proton density distribution $\left(n_{p} / n_{p}^{\text {tot }}\right)$ in $\left[\mathrm{cm}^{-3}\right]$ of the $1^{\text {st }}$ and $6^{\text {th }}$ of the detector irradiated by $10 \mathrm{MeV}$ proton beam. The surface is measured at $13.5 \%$ of the maximum value. The volume was divided in 160 x 160 uniformed voxels with a volume of $60 \mu \mathrm{m}(\mathrm{dx})$ x $60 \mu \mathrm{m}(\mathrm{dy}) \mathrm{x} \mathrm{dz}$ with dz the scintillator thickness. The lateral scattering is estimated about $2 \mathrm{~mm}$. 


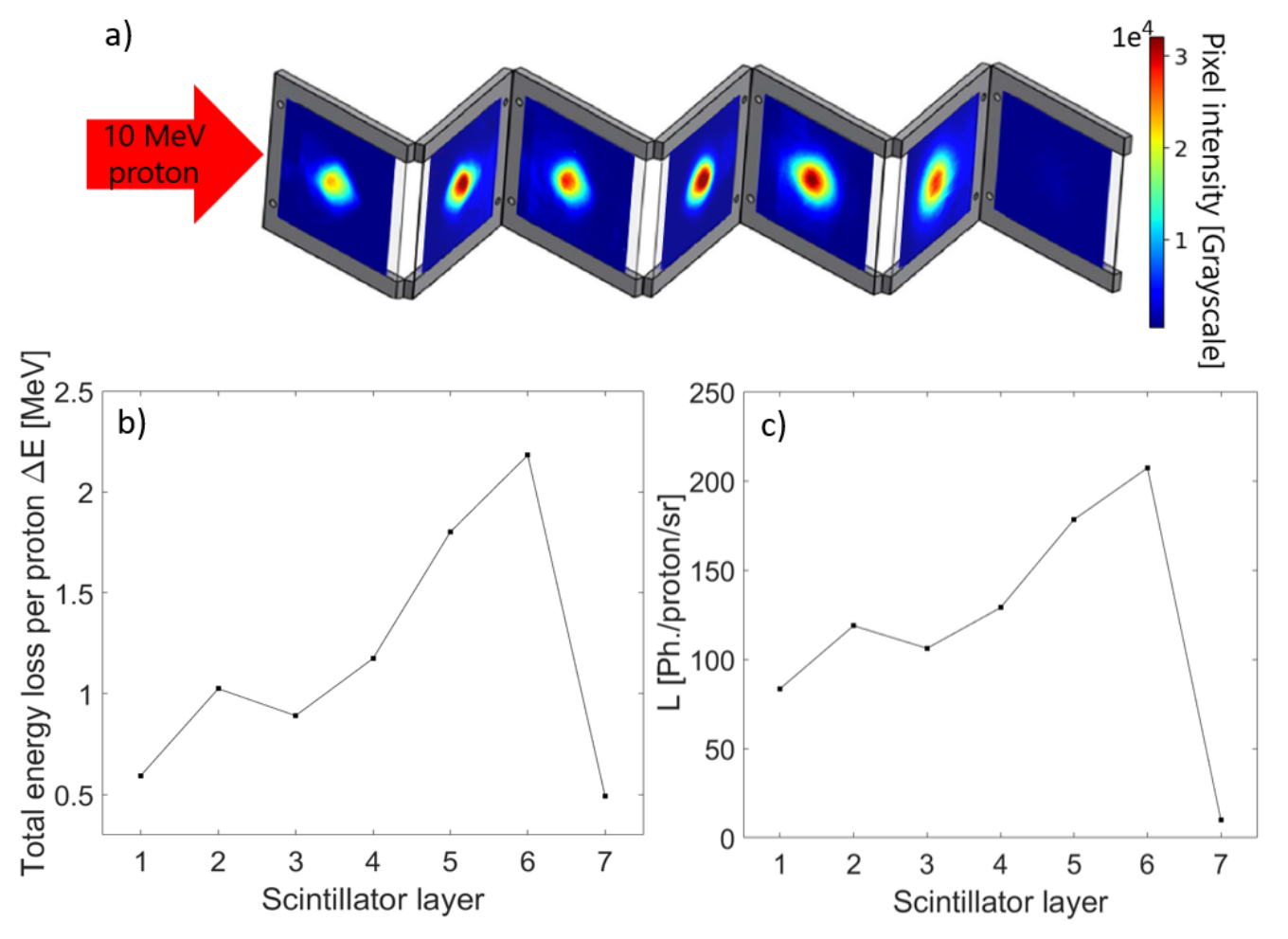

Figure 4.18: (a) Experimental signal obtained by the CCD camera during the irradiation with a $10 \mathrm{MeV}$ proton beam with the color scale giving pixel values artificially overlaid on a 3 dimensional representation of the detector. (b) Example of Monte Carlo simulation (obtained with FLUKA) representing the transversally integrated deposited energy per particle for each scintillator layer irradiated by a $10 \mathrm{MeV}$ proton beam. (c) represents the response of the scintillator (light output) to $10 \mathrm{MeV}$ proton beam irradiation. We can observe a peak of energy in the layer 2 (fig b and c) due the thickness difference between the layer 2 and $3(180 \mu \mathrm{m}$ against $140 \mu \mathrm{m})$. Thus, far from the Bragg peak, a proton will deposit more energy in the layer $n^{\circ} 2$ than in the layer $n^{\circ} 3$. Each layer (S) has a different thickness (this comes from the uncontrollable variation during the fabrication process of the company) and was estimated with a high precision caliper: $\mathrm{S}_{1}=120 \mu \mathrm{m} ; \mathrm{S}_{2}=180 \mu \mathrm{m} ; \mathrm{S}_{3}=140 \mu \mathrm{m} ; \mathrm{S}_{4}=160$ $\mu \mathrm{m} ; \mathrm{S}_{5}=190 \mu \mathrm{m} ; \mathrm{S}_{6}=130 \mu \mathrm{m} ; \mathrm{S}_{7}=150 \mu \mathrm{m}$ with an incertitude of $\pm 10 \mu \mathrm{m}$ (lines in fig $\mathrm{b}$ and $\mathrm{c}$ are visual guides and not fits). 


\subsection{Implementation of the detector in laser-driven pro- ton acceleration experiment at the HZDR facility}

The second experimental campaign consisted of testing the capabilities of our detector under extreme conditions of laser-plasma interaction. Under such conditions, the detector is exposed to a hostile environment close to the target, such as debris ejected from the target, direct damage from laser beam or electromagnetic pulses generated during high-intensity laser-plasma interaction experiments, which may affect the electrical circuit of diagnostic. In addition, other particles such as electron and ions as well as X-rays generated during the interaction can play a role as background noise in the deconvolution of the signal. The aim of the collaboration with the HZDR was to obtain a transfer of know-how on the development, configuration and application of on-line scintillator detectors between the two laboratories. Indeed, the HZDR team is known for its competence in this field with its successful applications of prototypes of scintillation detectors [Metzkes et al., 2012, 2016] . In addition, the HZDR center can provide proton acceleration source reaching cut-off energies up to $20 \mathrm{MeV}$ with their high power laser system Draco I [Obst et al., 2017]. Such performances are therefore perfect for testing the dynamic range of our detector.

\subsubsection{DRACO LASER SYSTEM}

The DRACO laser (the Dresden Laser Acceleration Source) is a dual beam system providing full Petawatt (30 J in $30 \mathrm{fs}$ ) and $150 \mathrm{TW}(4.5 \mathrm{~J}$ in $30 \mathrm{fs}$ ) after compression using CPA technique with optimized temporal pulse contrast [Obst et al., 2018] and high beam quality. In the framework of this thesis, only the $150 \mathrm{TW}$ arm is concerned. The laser is based on the PULSAR laser system provided by Amplitude Technologies and uses titanium doped sapphire (Ti:Sa) crystals as gain medium at a central wavelength of $800 \mathrm{~nm}$ and a pulse a pulse bandwidth of up to $80 \mathrm{~nm}$. A deformable mirror is used for wavefront optimization operating in a closed loop with a sensor (PHASICS SID4) situated at the experimental cave. Further details can be find in the Appendix B.

\subsubsection{Experimental set-up}

After final pulse compression and wavefront correction, the p-polarized laser pulse entered the experimental chamber and was focused with a maximum laser energy of $2.9 \mathrm{~J}\left(\mathrm{I}_{L} \approx 10^{21} \mathrm{~W} . \mathrm{cm}^{-2}\right)$ onto a $2 \mu \mathrm{m}$-thick titanium foil target with $45^{\circ}$ incidence angle. Figure 4.19 represents the experimental chamber. The laser beam was provided by two mirrors M1 and M2 and was focused by an off-axis parabolic mirror (OAP) F/2.5 with dielectric coating, $23.5^{\circ}$ off-axis angle and $25 \mathrm{~cm}$ focal length. Optimization and imaging of the focal spot at the millijoule level is performed with a dedicated long-distance microscope objective with a focal length of $100 \mathrm{~mm}$ corrected for spherical and chromatic aberrations. The resulting focal spot was about $3.5 \mu \mathrm{m}$ diameter (FWHM). 


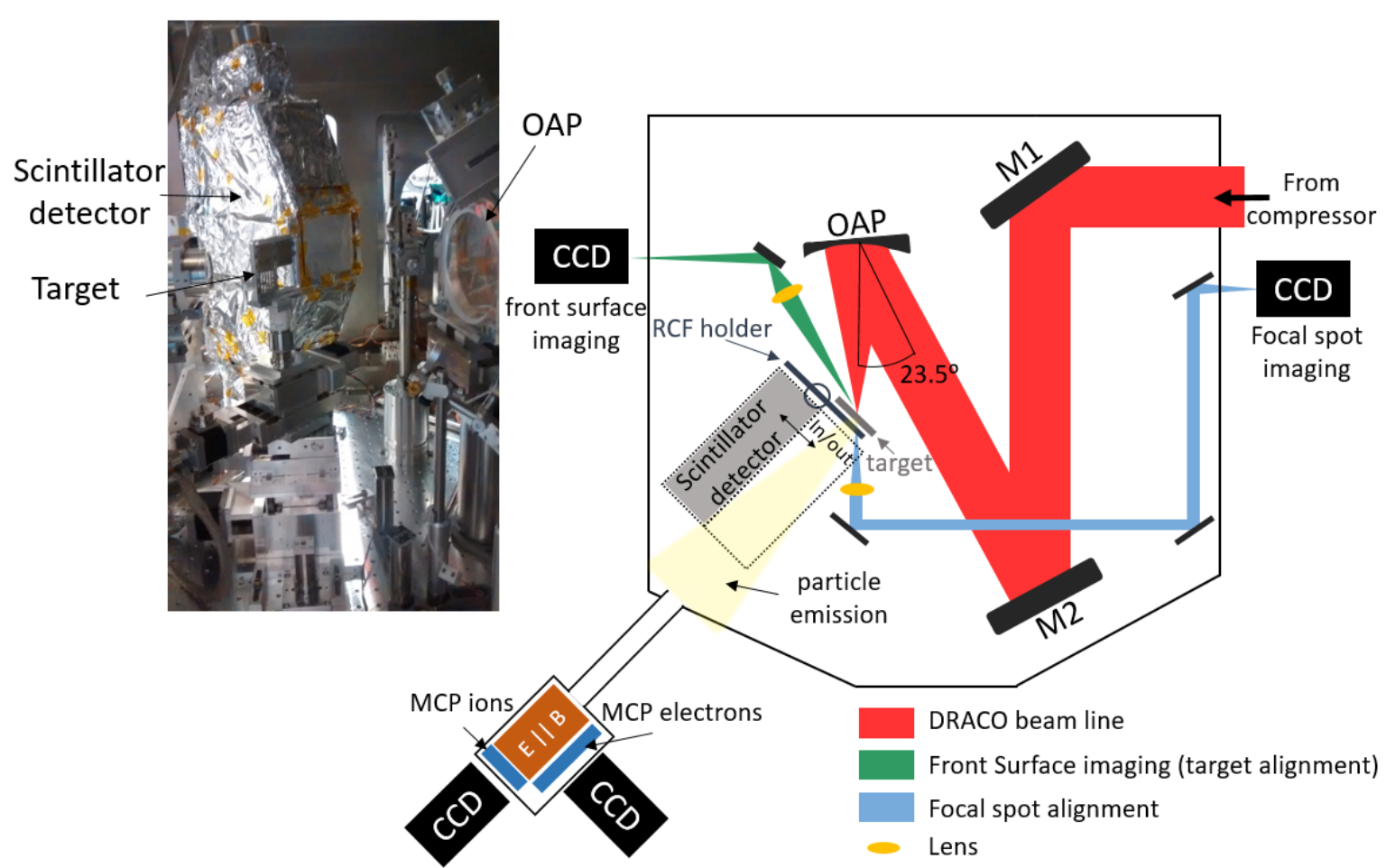

Figure 4.19: Illustration of the experimental setup for the first test of the scintillator detector with laser-driven proton acceleration at the Draco I laser system. Complementary diagnostics were used during the experiment: i) the RCF placed in front of the scintillator detector at $2.7 \mathrm{~cm}$ downstream from the target ii) A combined spectrometer for ion and electron detection placed at $80 \mathrm{~cm}$ downstream from the target when the scintillator detector was out. Illustration inspired by [Metzkes, 2015].

\section{Target Alignment}

A $2 \mu \mathrm{m}$ thick Ti target was placed inside a sandwich-type layer containing 70 holes for shots. Each hole of the layer has a rectangular dimension of approximately $0.8 \times$ $2 \mathrm{~mm}$, spaced from each other by $\approx 0.8 \mathrm{~mm}$ (vertical) and $\approx 1.8 \mathrm{~mm}$ (horizontal). During this experiment, two sandwich type layers were inserted into the upper part of a support (see figure 4.20) while the lower part was used for target alignment. Firstly, a pin (red dashed line on Figure 4.20) is moved every day via the calibrated motor stages at the focus position to center the imaging system position. A transparent foil (yellow dashed line on Figure 4.20) is then illuminated with a cw laser and placed at focus position using the focal spot imaging system. Once it is on focus, an alignment laser co-propagating with the DRACO beam is sent onto the foil and imaged with the front surface imaging system. The spot position on the front side camera is then the reference for a foil in focus and the non-transparent titanium foils are then placed 
in focus via this reference.

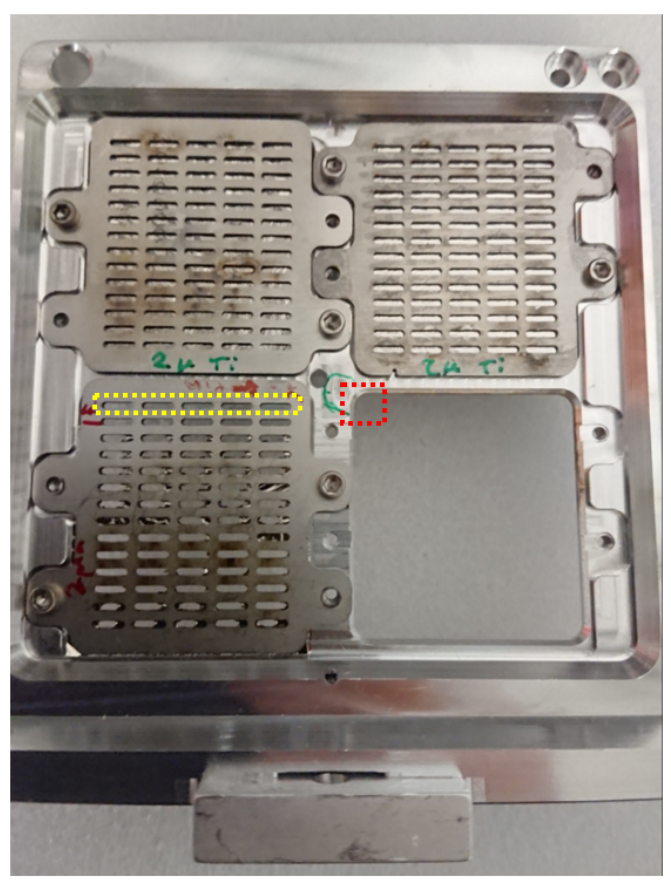

Figure 4.20: Target holder used during the experiment.

\section{Scintillator detector}

The design of the detector was split in two parts, one for the imaging system, carried by the HZDR team and another one for the detector design and composition, fulfilled by the CLPU team. The imaging system was composed by CCD Guppy PRO F-201 monochrome with a sensor resolution of $1624(\mathrm{H}) \times 1234(\mathrm{~V})$ and pixel size of $4.4 \mu \mathrm{m}$ coupled with an objective Navitar $8 \mathrm{~mm} \mathrm{~F} / 1.4$ 2/3 High Resolution. In order to work under vacuum condition, CCD and objective were placed inside the interaction chamber and housed in the CCD camera unit containing a small air volume to cool the camera. The detector part was designed and built assuming some spatial limitation from the interaction chamber and according to previous results obtain by the HZDR facility concerning proton emission distribution [Obst et al., 2017]. The detector was composed by ten square scintillator layers BC-400 of $54 \mathrm{~mm} \times 54 \mathrm{~mm}$, each of them separated by a relative angle of $25^{\circ}$. The first seven layers had a stated thickness of $100 \mu \mathrm{m}$ and the last three $250 \mu \mathrm{m}$ in order to compensate the signal reduction inversely proportional to the square of the distance from the source and the lower particle number at high energy (see table 4.4). Absorbers of different thicknesses were placed on the front face (upstream) of each scintillator in order to customize the energy resolution of the detector. Each absorber consisted of an aluminum filter 
of variable thickness surrounded on each side by a lexan-type opaque material with a fixed thickness of a few microns, in order to isolate the signals emitted by each layer. The scintillation emission was collected from the rear side of each scintillator (downstream side) simultaneously by two imaging system units. One imaged the 5 odd layers from one side (imaging system 1 ) and another one imaged the 5 even layers (imaging system 2), at the same time (Figure 4.21). Filter holders could be placed in the output of the scintillator layers to adapt the signal brightness read by the CCD. The composition of the detector was made in such a way that it could detect proton energies from 4.05 up to $20.1 \mathrm{MeV}$. The detector was placed at $3.8 \mathrm{~cm}$ downstream from the target. The detection solid angle of the CCDs for each layer was estimated by using a tritium radioluminescence source. By this manner, one could get the solid angle relation between each layer. It is then enough to know the solid angle of detection of a single layer to deduce the other ones.

\begin{tabular}{c|cccccccccc}
\hline \hline \multicolumn{10}{c}{ Proton kinetic energy range in the Scintillator diagnostic } \\
\hline Layer & 1 & 2 & 3 & 4 & 5 & 6 & 7 & 8 & 9 & 10 \\
\hline$E_{k}[\mathrm{MeV}]$ & 4.1 & 6.5 & 8.4 & 10 & 12 & 13.6 & 15.2 & 16.6 & 18.4 & 20.1 \\
\hline$\Delta E[\mathrm{MeV}]$ & 2.4 & 1.9 & 1.6 & 2 & 1.6 & 1.1 & 2.4 & 2.4 & 2.4 & 2.4 \\
\hline$* E_{k}=$ minimum proton kinetic energy reaching the layer & & & \\
$\Delta E=$ Energy resolution
\end{tabular}

Table 4.4: Proton kinetic energy range in each BC-400 scintillator layer. The estimation of the energy loss and minimum kinetic energy was estimated with FLUKA Monte Carlo code. Each layer was set with the stated thickness specified by the manufacturer. However, one can see that the layer $n^{\circ} 4$ has a bigger energy range compare to previous layers. Indeed, the signal obtain from the layer $4^{\text {th }}$ showed a strong increase compared to other layers, meaning that the thickness was probably bigger. We thus set the thickness of layer $\mathrm{n}^{\circ} 4$ considering the $30 \%$ error provided by the manufacturer. 


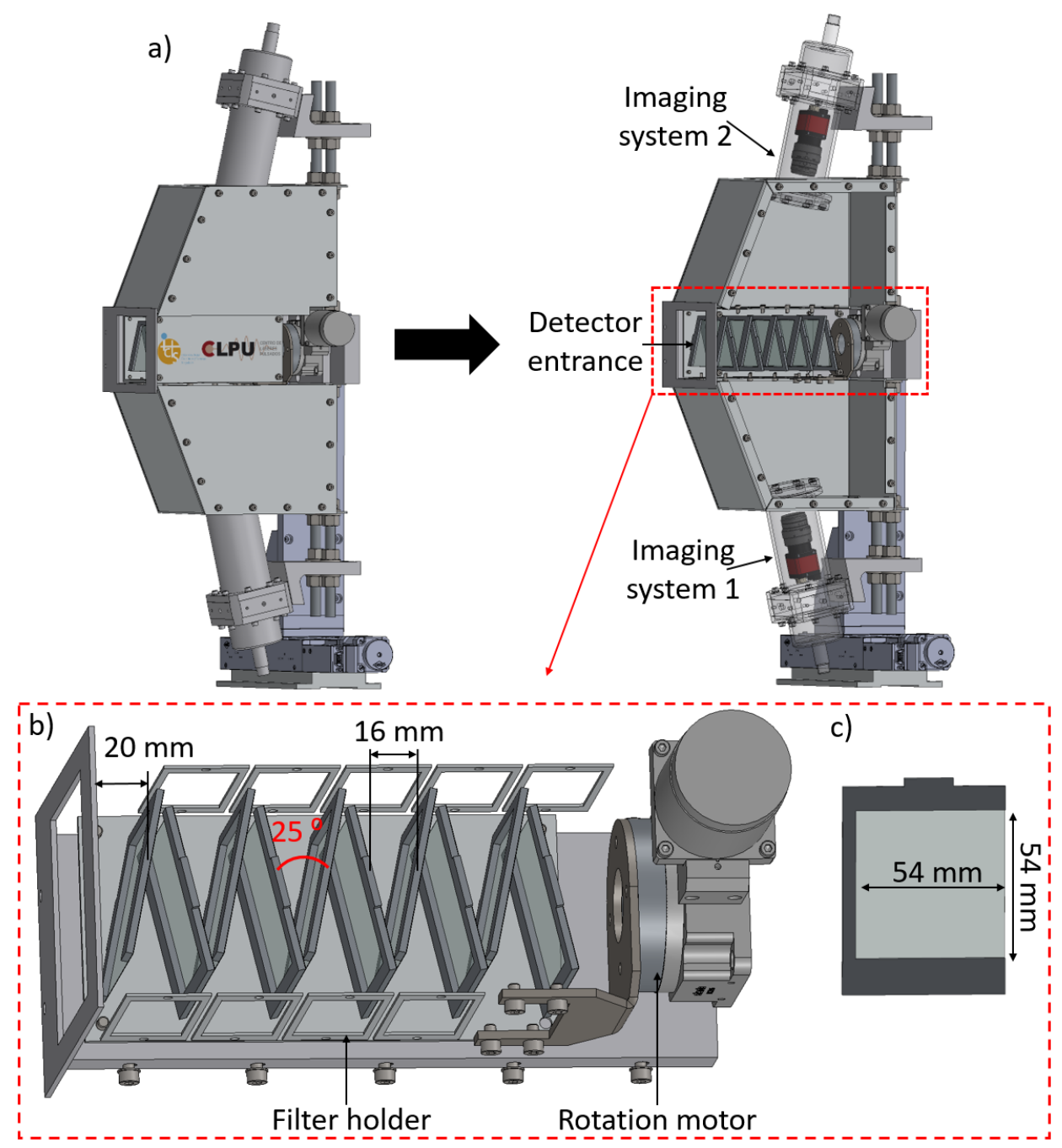

Figure 4.21: 3D-draw of the scintillator detector. The detector is composed by 10 square scintillator layers BC-400 of $54 \mathrm{~mm} \times 54 \mathrm{~mm}$. The layers are spaced $16 \mathrm{~mm}$ apart, with a relative angle of $25^{\circ}$ between each other. The odd layers $1,3,5,7$ and 9 are imaged by the imaging system 1 and the even layers 2, 4, 6, 8 and 10 by the imaging system 2. CCD and objective are housed in the CCD camera unit containing a small air volume to cool the system. Filters can be added to the scintillator signal output to avoid saturation of the read output signal. A rotation motor was fixed in the rear side of the detector to facilitate this change. a) outside and inside view of the whole detector composed by the scintillator in the central part, the two imaging systems on top and bottom part surrounded by a metallic shielding. b) zoom on the central part composed by the 10 scintillator layers. c) dimension of one scintillator layer. 


\section{Complementary diagnostics}

Additional diagnostics were used to cross check the results obtained with the scintillator detector. The energy-resolved spatial distribution of the accelerated proton beam was measured with stacks of RCF $(1 \times$ Gafchromic HD-810 $+10 \times$ EBT3 [Gafchromic, 2020]) able to resolved proton energies up to $20.6 \mathrm{MeV}$. The energy resolution was limited by the thickness of the film material and was equal to $\approx 1 \mathrm{MeV}$ for proton kinetic energy above $15 \mathrm{MeV}$ (see Table 4.5). The HD-810 film, coupled with a $13 \mu \mathrm{m}$ aluminum cover of the stack was also used as absorber for the possible ions generated. The stacks were placed at $2.7 \mathrm{~cm}$ downstream from the target and were made of half part or with a central hole of $4 \mathrm{~mm}$ to allow simultaneous measurements with the scintillator detector. Our detector was moved out of the proton propagation axis with a linear stage motor in order to let the central part of the particle beam propagates into a Thomson Parabola spectrometer (TP) placed at $80 \mathrm{~cm}$ downstream from the target (see Figure 4.19). The magnetic and electric fields were used to detect the ion species and spectra on the rear part of the spectrometer and electrons were obtained as well with the magnetic deflection on the side part of the spectrometer. Both signals were recorded using (MCP) detectors coupled with fluorescent screens and were read out with CCD cameras.

\begin{tabular}{c|cccccccccc}
\hline \hline \multicolumn{10}{c}{ Proton kinetic energy range in the RCF diagnostic } \\
\hline Layer & 1 & 2 & 3 & 4 & 5 & 6 & 7 & 8 & 9 & 10 \\
\hline$E_{k}[\mathrm{MeV}]$ & 4.7 & 7.1 & 9 & 10.6 & 12.1 & 13.4 & 14.6 & 15.7 & 16.8 & 17.8 \\
\hline$\Delta E[\mathrm{MeV}]$ & 2.4 & 1.9 & 1.6 & 1.5 & 1.3 & 1.2 & 1.1 & 1.1 & 1 & 1 \\
\hline${ }^{*} E_{k}=$ minimum proton kinetic energy reaching the layer & & & \\
$\Delta E=$ Energy resolution
\end{tabular}

Table 4.5: Proton kinetic energy range in each EBT3 layer.

\subsubsection{Proton spectrum reconstruction with the scintillator detector}

Although the design of the scintillator detector, with its angle and space between each layer, is different from that of RCFs, the method for obtaining the proton spectrum is the same as for RCF. Some notations in this analysis procedure follow the ones exposed in [Kaufman et al., 2015]. The detector is designed in such a way as to fully absorb the proton beam produced by the laser pulse interaction with the target. However, during the measurement, other type of particles will be accelerated during the interaction such as electrons and heavier ions as well as x-rays. Heavy ions can be neglect as first approximation since the propagation range of protons is much higher than that of heavier ions in a material, so that the scintillation emission generated by the protons is probably not affected by the heavier ions one. Considering that the background generated by electrons and X-rays is more or less homogeneous, their contribution can be suppressed by subtracting the background noise on each scintillator 
layer around the region that has the proton signal. We note that the noise correction is a common problem that RCF diagnostic encounters as well, where electron and $\mathrm{X}$-ray signal are generally interpreted as background noise around the proton area. For the proton spectrum reconstruction, each scintillator layer $i$ of the detector is associated with proton of energy interval $E_{i}$. Since the protons propagating through the detector deposit their energy all along the scintillator layers until they stop, the scintillation signal $L_{i}$ detected in the $i$-th layer originates from the contribution of all protons with kinetic energy $E_{k} \geq E_{i}$. However, this contribution can be separated in two parts (see Figure 4.22):

- Primary contribution in layer $i$ consider proton depositing their energy in the scintillator layer $i$ and stopping at a depth $z<z_{i+1}$ i.e before reaching the layer $i+1$. The corresponding protons have kinetic energies interval $E_{k} \in\left[E_{i}, E_{i+1}[\right.$.

- Secondary contribution in layer $i$ consider proton depositing their energy in the scintillator layer $i$ and stopping at a depth $z \geq z_{i+1}$ i.e stopping in layer $i+1$ or father. The corresponding protons have kinetic energies $E_{k} \geq E_{i+1}$.

We note that the scintillation emission was obtained by converting the 16-bits grey scale pixel values of the CCD output image into photon number using equation (4.9). The scintillation signal detected by the imaging system in the $i$-th layer can be written

$$
L_{i}^{\mathrm{tot}}=L_{i}^{(1)}+L_{i}^{(2)}
$$

with $(1)=$ Signal generated by primary proton in layer $\mathrm{i}$

and $(2)=$ Signal generated by secondary proton in layer $i$.

In our model, the selected area chosen for analysis is the whole observed proton beam in order to get the spatially integrated spectrum. The signal $L$ is thus the sum of the signal inside the selected area.

In order to get the proton energy distribution assumption $(\Delta N / \Delta E) i$, one must subtract the signal generated by the secondary protons so that only the contributions from the primary protons remain such as

$$
L_{i}^{(1)}=L_{i}^{(t o t)}-L_{i}^{(2)}
$$

The secondary contribution in the $i$-th layer can be express as a function of the signal generated by the primary protons of the layers of higher index $i$. Thus, equation (4.12) reads

$$
L_{i}^{(1)}=L_{i}^{(t o t)}-\sum_{j=i+1}^{N_{L}} L_{j}^{(1)} \frac{c_{i j}}{c_{j j}}
$$




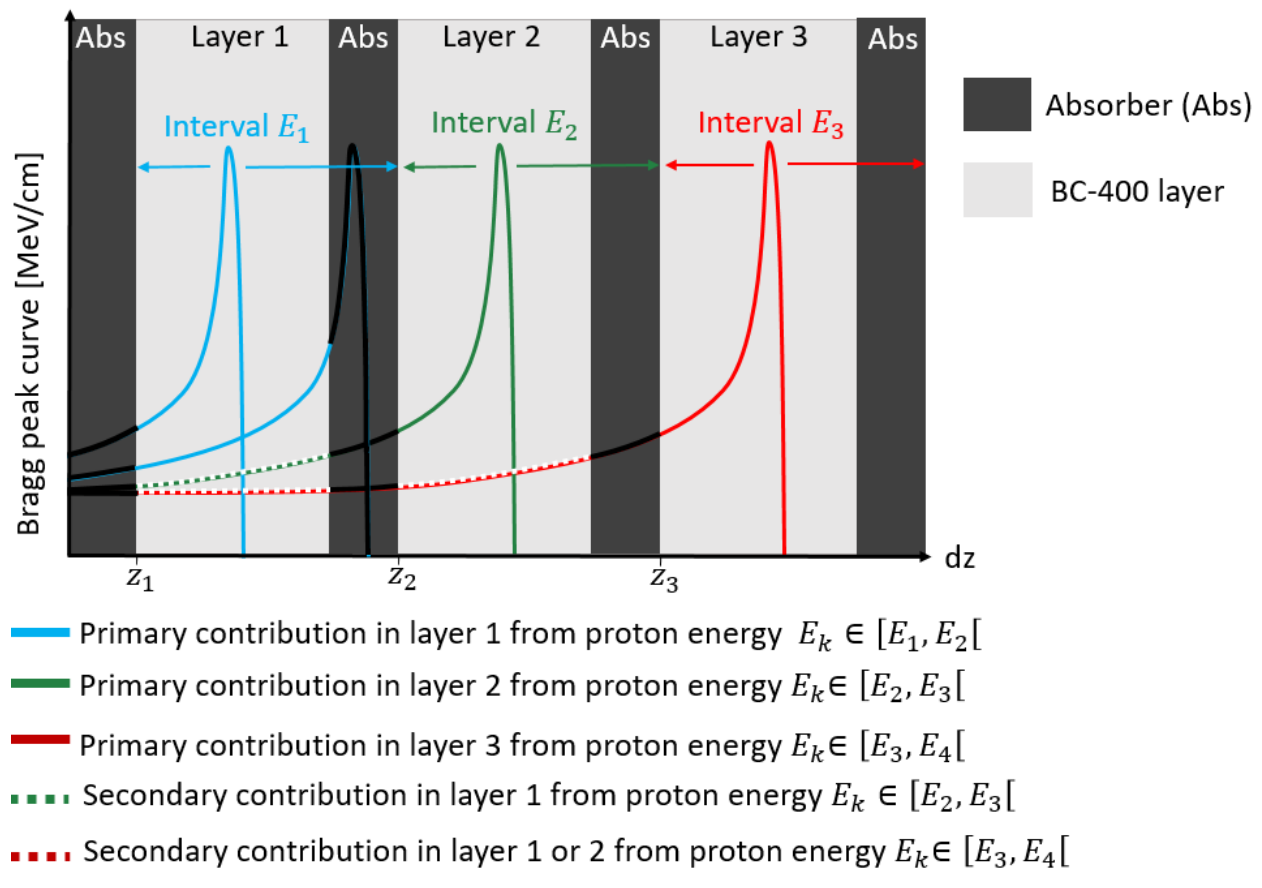

Energy deposited in the absorbers is not taking into account for the spectrum reconstruction

Figure 4.22: Illustration of the model for the spectrum reconstruction. Primary contribution in layer $i$ consider protons depositing their energy in the scintillator layer $i$ and stopping at a depth $z<z_{i+1}$ i.e before reaching the layer $i+1$. Secondary contribution in layer $i$ consider protons depositing their energy in the scintillator layer $i$ and stopping at a depth $z \geq z_{i+1}$ i.e stopping in layer $i+1$ or farther. For simplicity, only 3 layers are represented.

with $N_{L}$ the total number of layers where protons have deposited their energy, $c_{i j}$ is a coefficient denoting the averaged light output $L$ generated by a proton of the $\mathrm{j}$-th energy interval group in the i-th layer.

$$
c_{i j}=\left\langle L_{i, j}^{(\operatorname{sim})}\right\rangle
$$

For each j-th group, various number of simulations $N_{s, j}$ were acquired with FLUKA Monte Carlo code to obtain the proton Bragg curves in the i-th layer. The kinetic energy step between each simulation was set to $\Delta E_{s}=50 \mathrm{keV}$. The light output generated for each proton of the $\mathrm{j}$-th group was obtained integrating the Birks equation 4.10 and summed with all simulation $N_{s, j}$ to obtained the total light output $L_{i, j}^{(s i m)}$. The average value was finally obtained by dividing over the number of simulations $N_{s, j}$.

Our algorithm starts by converting the photon signal from the last layer $\left(i=N_{L}\right)$ into a number of primary proton thanks to its conversion coefficient $C_{i i}$. This num- 
ber of protons is then converted back to a number of photons and subtracted in all the preceding layers with different conversion coefficient for each layer. Then, the algorithm do the same procedure again, starting with the penultimate layer $\left(N_{L}-1\right)$ and so on.

Finally, the total number of primary protons in the $i$-th layer reads

$$
N_{i}=\frac{S_{i}^{(1)}}{c_{i i}} .
$$

Dividing (4.16) by the energy interval width $\Delta E_{i}$, the corrected histogram step-like spectrum $(\Delta N / \Delta E)_{i}$ is obtained.

\section{Validation of the method}

In order to validate our proton spectrum reconstruction technique, we have generated realistic grey value pixel intensity maps of $3 \times 3 \mathrm{~cm}^{2}$ (or $500 \times 500$ pixels) similar to those obtained with our detector on the CCD output images.

1. First, we created a proton beam with a proton spectrum distributed over an entire $3 \times 3 \mathrm{~cm}^{2}$ map. For simplicity, we assume a finite, Gaussian, monodirectional and cylindrical proton beam propagating along the $z$-axis. We note

$$
f_{p}\left(x, y, E_{k}\right)=N_{\text {tot }} f_{x}(x) f_{y}(y) f_{E}\left(E_{k}\right)
$$

its distribution function per unit of surface (transverse to the $z$-axis) and unit of kinetic energy just before interacting with our detector. $N_{\text {tot }}=10^{8}$ is the total number of protons.

$$
f_{x}(x)=\frac{1}{\sqrt{2 \pi \sigma_{x}^{2}}} \exp \left[-\frac{\left(x-x_{0}\right)^{2}}{2 \sigma_{x}^{2}}\right] \text { and } f_{y}(y)=\frac{1}{\sqrt{2 \pi \sigma_{y}^{2}}} \exp \left[-\frac{\left(y-y_{0}\right)^{2}}{2 \sigma_{y}^{2}}\right]
$$

with $\sigma_{x}=0.5 \mathrm{~cm}, \sigma_{y}=0.5 \mathrm{~cm}$ and $x_{0}=y_{0}=1.5 \mathrm{~cm}$ are the proton beam spatial distribution along the $x$ and $y$-axis respectively.

$$
f_{E}\left(E_{k}\right)=\left\{\begin{array}{cl}
\frac{\exp \left(-\frac{E_{k}}{k_{\mathrm{B}} T_{p}}\right)}{k_{\mathrm{B}} T_{p}\left[1-\exp \left(-\frac{E_{\mathrm{k}, \max }}{k_{\mathrm{B}} T_{p}}\right)\right]} & \text { if } \quad E_{k} \leq E_{\mathrm{k}, \max } \\
0 & \text { else }
\end{array}\right.
$$

is the proton beam kinetic energy spectrum assumed to be homogeneous in space with $T_{p}=1.5 \mathrm{MeV}$ and $E_{k, \max }=15$. The spatial area distribution of the proton beam is represented in Figure 4.23 and reads

$$
N(x, y)=N_{\text {tot }} \times f_{x}(x) \Delta x \times f_{y}(y) \Delta y
$$


with the respective spectral kinetic energy distribution

$$
N\left(x, y, E_{k}\right)=N(x, y) \times f_{E}\left(E_{k}\right) \Delta E
$$

where $\Delta x \times \Delta y$ correspond to the typical pixel size of an image record with our imaging system ( here $\Delta x=\Delta y=60 \mu \mathrm{m}$ ) and $\Delta E=50 \mathrm{keV}$ the energy range.

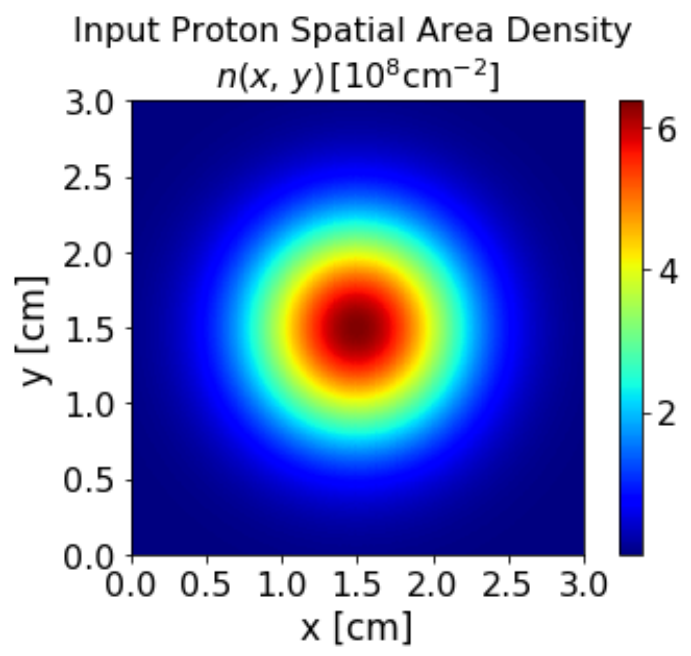

Figure 4.23: Spatial Area density of protons where $n=N / \Delta x \Delta y$.

The spatially-integrated proton beam kinetic energy spectrum is represented in figure 4.24 and read

$$
N\left(E_{k}\right)=N_{\text {tot }} \times f_{E}\left(E_{k}\right) \Delta E .
$$

2. We obtained with FLUKA simulations the Bragg curves of each proton with kinetic energy from 0 up to $E_{k}=E_{k \text {,max }}$ with a step $\Delta E$ propagating in each layer of the detector.

3. The light output (photon number) generated in each scintillator layer volume element $\Delta x \Delta y \Delta z$ ( $\Delta z$ is the layer thickness) is then evaluated with the Birks equation (4.10) using the quenching coefficients estimated in the previous section. Finally, the number of photons in each volume element is converted into 16-bits grey scale pixel intensity value thanks to the conversion equation (4.7).

4. The spatially-integrated proton spectrum is then obtained following the steps of the algorithm mentioned previously. It is represented in Figure 4.25.

The proton spectrum obtained with our algorithm is in agreement with the spectrum imposed as input. However, although this test can allow us to validate our spectrum 


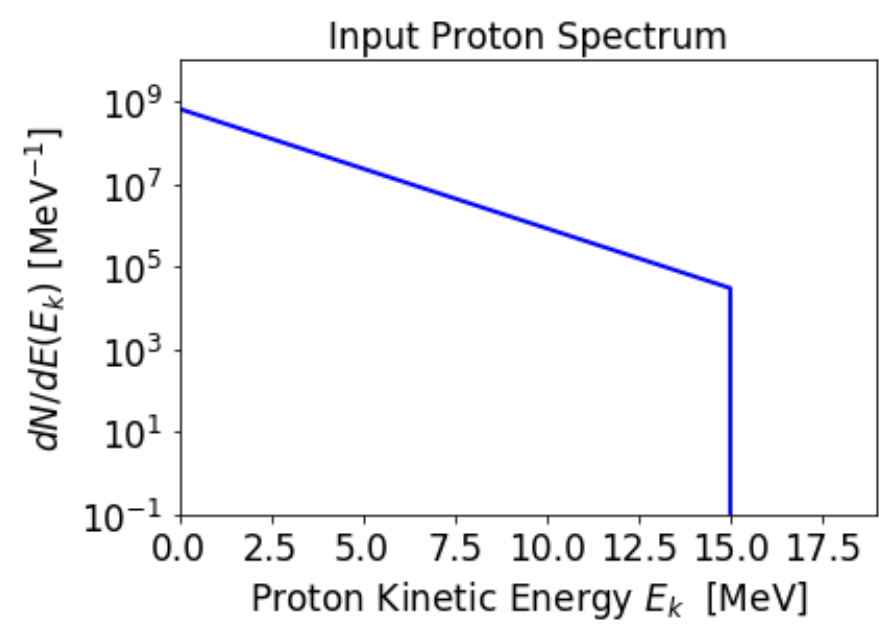

Figure 4.24: Spatially integrated proton spectra generated for the test case.

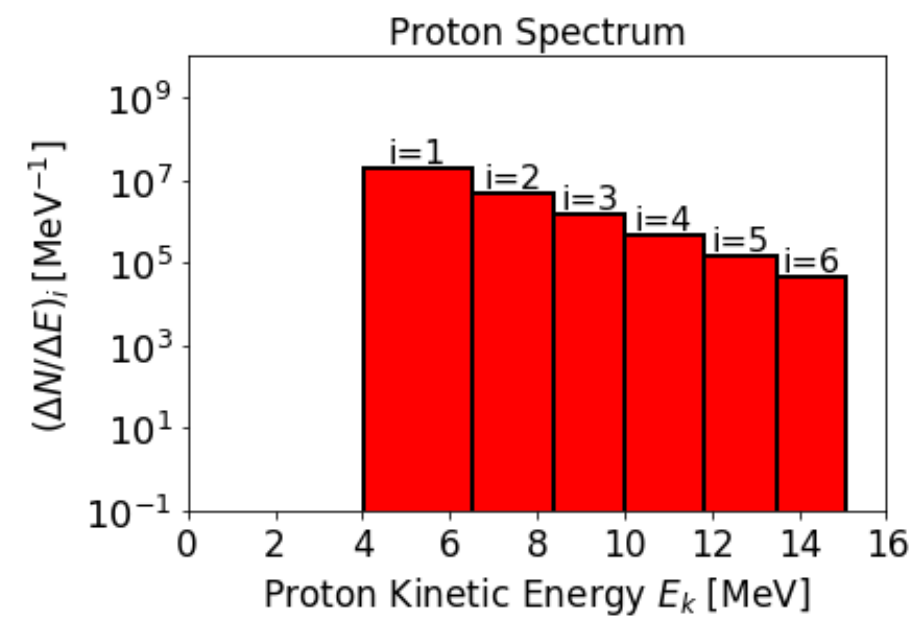

Figure 4.25: Proton spectra of the spatially-integrated proton beam obtained with our algorithm.

reconstruction technique, it does not allow to estimate the errors which could have been made on the quenching parameters evaluation or on the CCD count conversion approximation.

\subsubsection{Proton beam divergence reconstruction}

The dependence of proton beam divergence and energy feature according to the laser parameters, target thickness and composition is crucial to improve the understanding of the acceleration mechanisms. Unlike the RCF diagnostic, which generally 
measures less than $2 \mathrm{~mm}$ in length, the scintillator detector can measure more than 10 $\mathrm{cm}$ in length, thus affecting the interpretation of the initial proton beam divergence. In order to reconstruct the original proton divergence in each layer of the scintillator detector, we developed a small algorithm which takes into account the phenomenon of scattering of a proton during its propagation in various materials.

Assuming that the proton detector is made by a series of parallel BC-400 scintillator layers with an infinite area, thickness $\Delta z$ and density $\rho$ and areal density $A_{s}=\rho \Delta x$. The detector is made by a series of i layers placed at a distance $L$ to each other. We suppose a monoenergetic proton beam source with an initial kinetic energy $E_{k, 0}$ placed at a distance $L_{0}$ upstream from the detector with a divergence half angle $\theta_{0}$. A proton of kinetic energy $E_{k, i}$ entering through the $(i+1)$-th layer loses a total amount of energy

$$
\Delta E_{k, i}=E_{k, i}-E_{k, i+1}
$$

and suffers from an angular elastic scattering according to equation (3.79)

$$
\theta_{i}^{R M S} \approx \frac{\sqrt{A_{s}}}{E_{k, i-1}}\left[1.14+\epsilon \log _{10}\left(A_{s}\right)\right]
$$

We note $F_{i}$ and $B_{i}$ the front and back surface of the $i$-th layer in 1D approximation. The Proton beam travel along the $\mathrm{z}$ axis.

The estimation of the front surface of the first layer reads

$$
F_{1}=2 L_{0} \tan \theta_{0}
$$

the proton beam entering the first layer will suffer from multiple scattering and the final deflection angle $\theta_{1}$ which is the sum of the initial divergence angle $\theta_{0}$ and the scattering angle from the first layer $\theta_{1}^{R M S}$. The final area at the back side of the first layer read:

$$
B_{1}=2 \Delta z \tan \theta_{1} \quad \text { with } \theta_{1}=\theta_{0}+\theta_{1}^{R M S} .
$$

In the same manner, front surface of layer 2 is given by

$$
F_{2}=B_{1}+2 L \tan \theta 1=F_{1}+2(\Delta x+L) \tan \theta_{1}
$$

Consequently, the proton surface back side of layer 2 can be expressed as:

$$
\begin{gathered}
B_{2}=F_{2}+2(\Delta x) \tan \theta_{2}=F_{1}+2(\Delta x+L) \tan \theta_{1}+2(\Delta x) \tan \theta_{2} \\
\text { with } \theta_{2}=\theta_{0}+\theta_{1}^{R M S}+\theta_{2}^{R M S}
\end{gathered}
$$

By iterating the process we can finally write:

$$
\begin{aligned}
& F_{i}=F_{1}+2(\Delta x+L) \sum_{j=1}^{i-1} \tan \theta_{j} \quad(\text { for } i \geq 2) \\
& B_{i}=F_{i}+2 \Delta x \tan \theta_{i} \quad \text { for } \\
& \theta_{q}=\theta_{0}+\sum_{p=1}^{q} \theta_{p}^{R M S} .
\end{aligned}
$$




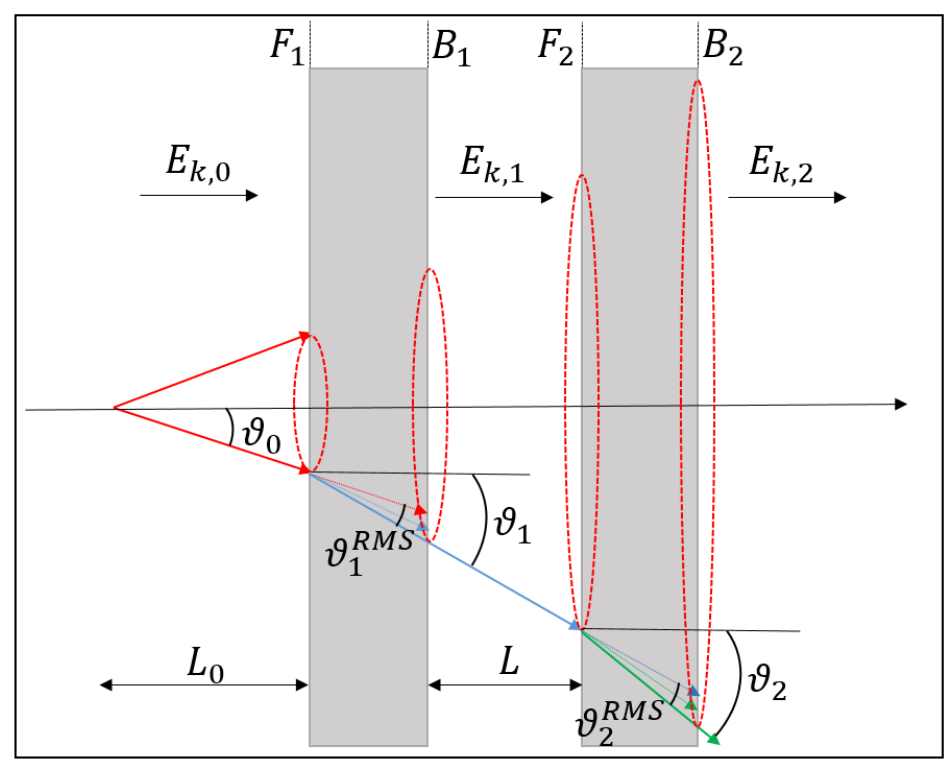

Figure 4.26: Proton half angle divergence model.

\subsubsection{Experimental results and discussion}

Electron spectra was recorded using the spectrometer. Figure 4.27 (left) shows the evaluated spectra data for a target of $2 \mu \mathrm{m}$ Ti and laser energy of $2.9 \mathrm{~J}$ on target. Due to the low signal-to noise-ratio of the signal, the electron spectrum for each day was averaging over all shots. The temperature of the fast electron beam population was obtained by fitting the data with the two-temperature exponential decay model of [Sawada et al., 2014] (3.34). We found $T_{h 1}=0.4 \pm 0.1 \mathrm{MeV}$ for the coldest electron population and $T_{h 2}=1.8 \pm 0.2 \mathrm{MeV}$ for the hottest electron population. The evaluated temperatures were averaged over the three day electron spectra in order to prevent fluctuations.

Figure 4.27 (right) represents the measurements of the relative proton energy spectrum with the TP for the best shots of each day. The proton cut-off energy was evaluated to $\approx 17 \mathrm{MeV}$ and is in agreement with the RCF diagnostic (see Figure 4.28). The proton spectrum obtained with the RCF diagnostic have been evaluated for two different days and one can observe a good stability with regard to the cut-off and population distribution of the accelerated proton beam.

Figure 4.29 represents the proton beam profiles obtained with the RCF diagnostic. By comparing the signal obtained by the RCF and the scintillator diagnostic for the same shot, one can observe that the proton beam becomes blurred due the scattering through the materials in the case of the scintillator diagnostic. In addition, the observed signal fades for weaker energies on the scintillator layers than on the RCFs. 

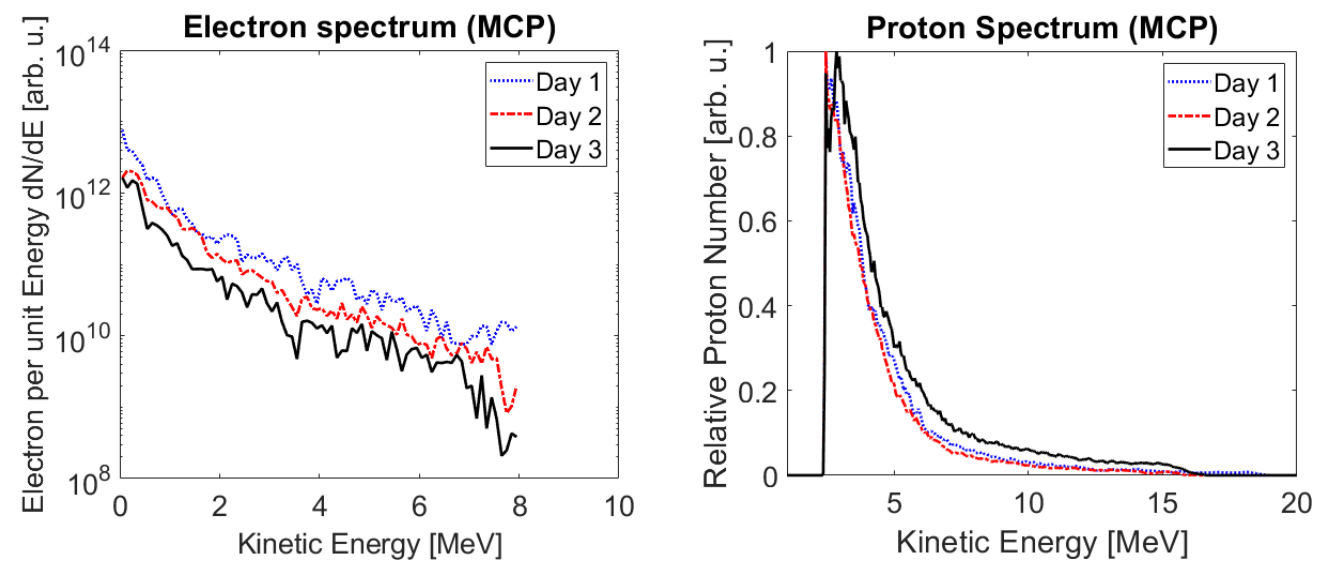

Figure 4.27: Electron (right) and proton (left) spectrum obtain with the TP. Due to the low noise-signal ratio, the electron spectrum was averaging over all shots for each day.
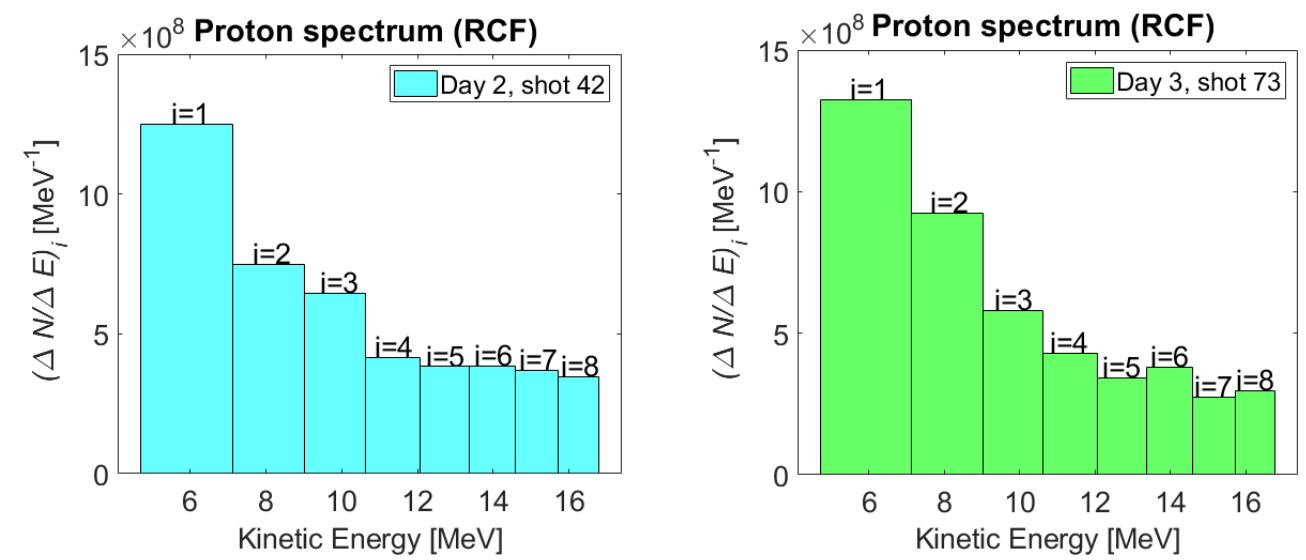

Figure 4.28: Proton spectrum obtained with half-RCF shape diagnostic for two different shots. The full spectrum for both shots was obtained by extrapolating the other half of the RCF.

Several reasons can be the cause of this behavior such as the low noise-signal ratio, the signal intensity reduction $\propto 1 / r^{2}$ with the distance $r$ and the reduced number of protons in the high energy tail of the exponential spectrum. Figure 4.30 represents the signal obtain on the CCD output image. One can clearly see a blurred signal fading strongly in the last layers. The estimation of the proton divergence according to our model described previously (section 4.4.4) was compared to the RCF one and is presented in Table 4.6. The divergence is averaged over two shots and measured along the horizontal axis (the vertical axis being cropped for layers 1 and 2 of the scintillator detector due to angle field problems) for both diagnostics (the shots are not the same for each diagnostic). The divergence evaluated with the scintillator seems 
a)
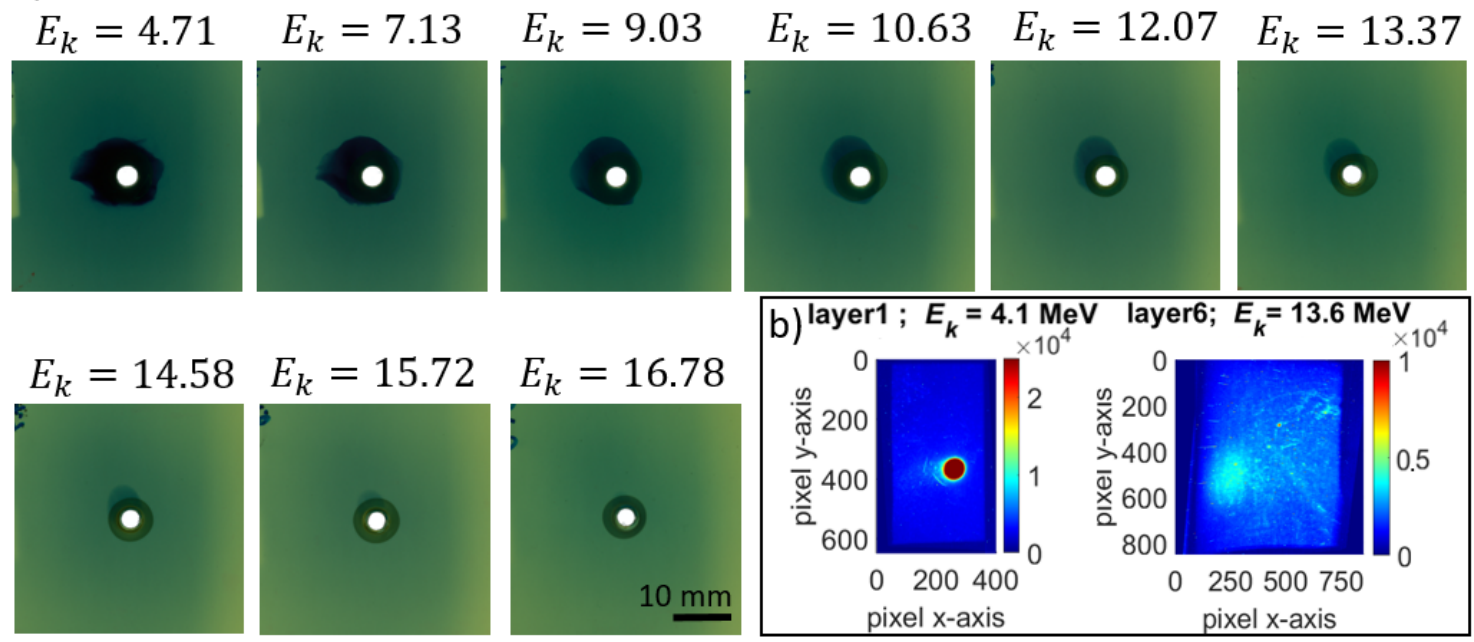

Figure 4.29: a)Proton beam profile measured with RCF diagnostic made with a 4 $\mathrm{mm}$ hole in the center. Proton signal is observe up to the $9^{\text {th }}$ layer corresponding to Kinetic proton energy $\geq 16.78 \mathrm{MeV}$. b) CCD output image of the proton beam propagating through the hole for the same shot on the scintillator detector. Proton signal is observe up to the $6^{\text {th }}$ layer corresponding to kinetic proton energy $\geq 13.6$ $\mathrm{MeV}$. Both groups: each layer represents the proton energy at or above the kinetic energy $E_{k}$.

to be underestimated, especially in the last layers. One of the main reason comes from the difficulty in selecting the correct whole beam due a weak and blurred signal mixed with the background signal in the last layers (see Figure 4.30). In addition, the model applied for the divergence reconstruction may overestimate the multiple scattering [Lynch \& Dahl, 1991], thus underestimating the initial proton beam divergence.

Table 4.6: Estimation and comparison of the proton beam divergence (full angle) for the RCF and scintillator diagnostic.

\begin{tabular}{|c|c|c|c|c|c|c|}
\hline layer & 1 & 2 & 3 & 4 & 5 & 6 \\
\hline$E_{k} \mathrm{RCF}[\mathrm{MeV}]$ & 4.7 & 7.1 & 9 & 10.6 & 12.1 & 13.4 \\
\hline$\phi \mathrm{RCF}\left[^{\circ}\right]$ & $24.4 \pm 0.9$ & $23.8 \pm 1.2$ & $23.1 \pm 1$ & $22.2 \pm 2$ & $18.2 \pm 3$ & $14.1 \pm 3$ \\
\hline$E_{k}$ Scint. $[\mathrm{MeV}]$ & 4.1 & 6.5 & 8.4 & 10 & 12 & 13.6 \\
\hline$\phi$ Scintillator $\left[{ }^{\circ}\right]$ & $24.3 \pm 1.1$ & $22.75 \pm 1.9$ & $20.8 \pm 1.8$ & $16.1 \pm 0.1$ & $12.9 \pm 0.1$ & / \\
\hline
\end{tabular}

${ }^{*} E_{k}=$ Minimum proton kinetic energy reaching the layer.

${ }^{*} \phi=$ Divergence of the proton source (full angle) 
a)
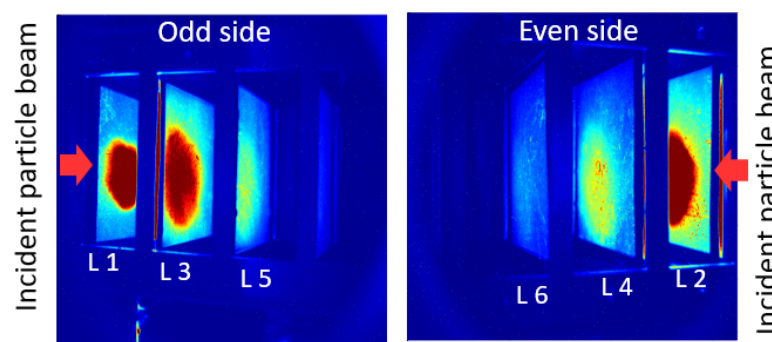

b)

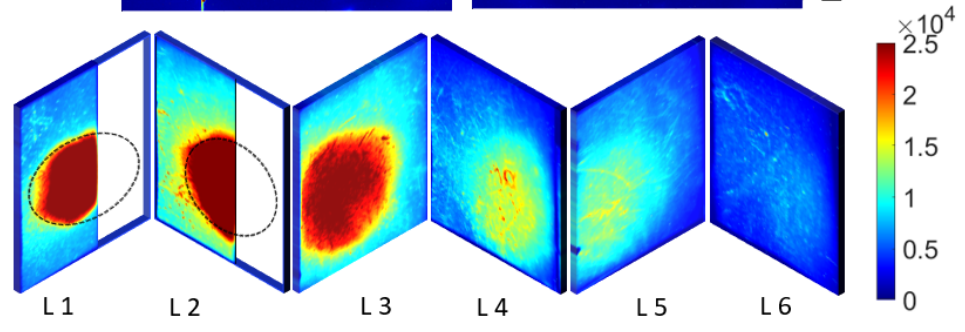

Figure 4.30: a) CCD output images from odd (left) and even (right) side. Both CCD output images show the back side of each scintillator layer. b) Reconstruction of the initial proton beam geometry taking into account the relative angle between the CCD and scintillator layer plan. Layer 1 (L 1) and 2 (L 2) were not fully visualized by the imaging system.

The proton spectrum evaluated with our algorithm is represented in Figure 4.31. By comparing the spectra obtained with the RCFs, we can observe that the distribution of the protons is greater by two orders of magnitude in the case of the scintillator. Several reasons can be the cause for such a discrepancy between diagnostics, such as uncertainties concerning the quenching coefficient, the solid angle, the cleaning of the background noise from other particles. In addition, the thickness of the scintillator can vary up to $30 \%$ of their original thickness according to the manufacturer and consequently acts on the determination of the conversion parameter $c_{i j}$ with Monte Carlo simulations.

\subsection{Conclusion}

Finally, as a proof of principle, the detector was successfully tested in a laser acceleration experiment and is capable to work at HRR, giving a direct feedback about the $2 \mathrm{D}$ spatial and energy resolution of the proton beam. Although adjustments are necessary in order to be able to use it as an absolute measurement diagnostic, we have demonstrated throughout the detailed analysis reported in this chapter the promising capabilities of such a diagnostic. We have shown that it is possible to account for the laser-driven proton divergence by maintaining a compact size of detector. Further developments of the detector are required regarding: 


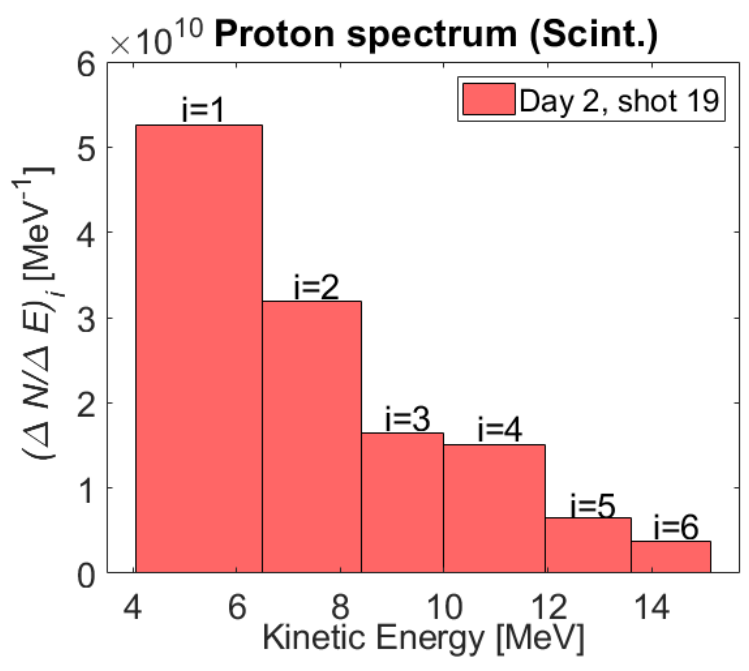

Figure 4.31: Proton spectrum obtained with the scintillator detector.

- The thickness layer measurement: such incertitude could be figure it out by measuring the surface roughness and possibly the thickness by interferometry techniques.

- The background noise suppression: part of the background comes from electrons. Most of the electron population contribution could be removed by implementing an additional permanent magnet in front of the detector, although the effect it has on the proton flux distribution at each energy will have to be mitigated. The light shielding/filter may be refined in order to improve the signal-noise ratio.

- The compactness of the detector: a compact detector will reduce the proton beam dimension in each layer and thus may improve the signal.

- Viewing system: the CCD output image may be improve by implementing individual viewing systems for each layer, thus improving considerably the spatial resolution of the diagnostic.

- Combination with other diagnostics: a central hole in the middle of the scintillator could be an option to measure simultaneously the proton signal on a TP detector. 


\section{Chapter 5}

\section{Application to Laser-driven proton acceleration with petawatt class laser system at CLPU}

Up to this point, we have explained the physics around laser-driven proton acceleration, described their interaction with matter and presented the main diagnostics used so far for their detection. We have then introduced in the previous chapter the first study on the development, testing and validation of a new scintillation detector for proton detection capable of operating at high repetition rate. The work presented in this thesis focus on the main objectives of the 5 years spent working within the CLPU. However, it is important to note that the CLPU is a recent facility that started to be operational for external users beginning of the year 2018. This last chapter is dedicated to the presentation of the commissioning experiment of VEGA-3 Petawatt (PW) laser system belonging to the CLPU facility. The experiment aims to show the capabilities of the VEGA-3 system for laser-driven proton acceleration on solid target for different laser parameters. Our scintillation detector has been implemented in the experiment set-up to test its capabilities and complementarity with other diagnostics. Finally, the preliminary results are presented.

\subsection{CLPU VEGA system}

The CLPU is a facility specialized in ultra short and ultra intense laser pulses. The main activity of the center is to provide high power laser to the scientific community with an open access to national and international users, as well as to promote scientific, technical and innovation development by collaborating with international laboratories. The main laser system of CLPU is the VEGA laser. Recently operative, VEGA laser is a three-beam system providing full Petawatt (30 J, 30 fs), 200 TW (6 
J, $30 \mathrm{fs}$ ) and $20 \mathrm{TW}$ (600 mJ, $30 \mathrm{fs}$ ) laser outputs. The laser is based on the PULSAR system from Amplitude Technologies and uses titanium doped sapphire (Ti:Sa) crystal as gain medium at a central wavelength of $800 \mathrm{~nm}$ with pulse bandwidth up to 70 $\mathrm{nm}$ (see Figure 5.1). The laser chain has a single and common front end which starts with the oscillator and uses a double-CPA architecture with a cross-polarized wave (XPW) pulse cleaning stage installed between the two CPA parts. In order to reduce pump induce distorsion, the Ti:Sa amplifiers use room-temperature water cooling and VEGA-2 main amplifier uses cryo-cooled crystal. A pockels cell before VEGA-3 preamp reduces the repetition rate from 10 to $1 \mathrm{~Hz}$ for PW arm. Every VEGA output has a fast shutter and pockels installed to control the operation rate from single shot to continuous $1 / 10-\mathrm{Hz}$ operation mode. A deformable mirror installed between the main amplifier output and the compressor of VEGA-3 is employed for beam quality enhancement with the associated wavefront sensor located in the metrology bench. Table 5.1 recapitulates the main properties of the VEGA laser system.

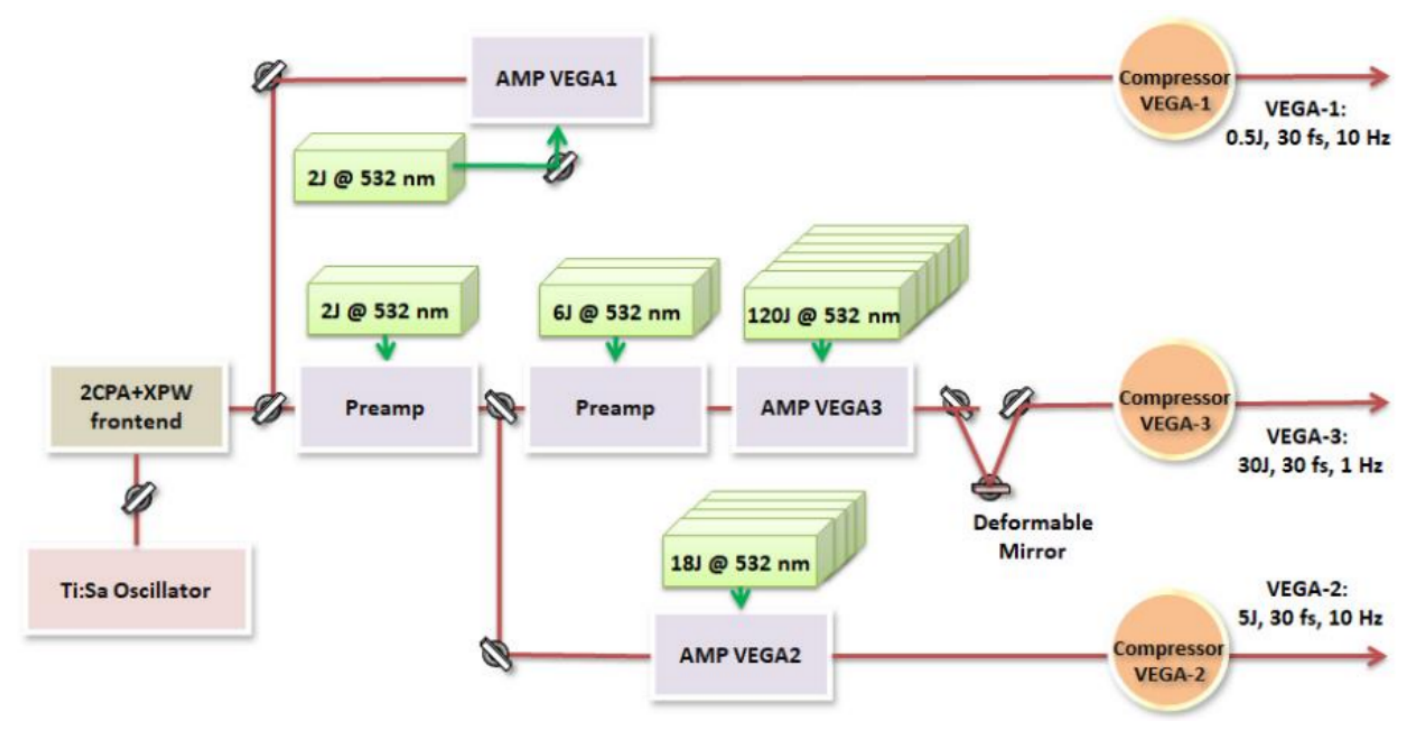

Figure 5.1: Main VEGA laser step lines. Illustration from [Méndez et al., 2019].

\subsubsection{Pulse contrast}

One relevant parameter of high-power laser systems for experiments on laser interactions with thin foils is the temporal intensity contrast quality. For ultra-intense sources, the temporal contrast at a delay $\Delta t$ is defined as the ratio between intensity of the main pulse and the intensity at a delay $\Delta t$. Usually, ultra-short femtosecond laser pulse is surrounded by a short pedestal native from an imperfect recompression, accompanied by a long pedestal introduced by the amplified spontaneous emission (ASE) and low intensity replicas of the main pulse situated at specific times before and after the main laser pulse, the so called pre and post-pulses. Typical values range 
Table 5.1: Main parameter of VEGA laser system installed at CLPU

\begin{tabular}{l|ccc}
\hline \hline Laser Properties & VEGA 1 & VEGA & VEGA-3 \\
\hline Beam diameter after compression & $35 \mathrm{~mm}$ & $110 \mathrm{~mm}$ & $220 \mathrm{~mm}$ \\
\hline Laser energy & $0.6 \mathrm{~J}$ & $6 \mathrm{~J}$ & $30 \mathrm{~J}$ \\
\hline Pulse duration & \multicolumn{3}{|c}{$30 \mathrm{fs}$} \\
\hline Peak power & $20 \mathrm{TW}$ & $200 \mathrm{TW}$ & $1 \mathrm{PW}$ \\
\hline Central wavelength and spectral bandwidth & \multicolumn{3}{|c}{$800 \pm 70 \mathrm{~nm}$} \\
\hline Repetition rate & \multicolumn{2}{|c}{$1-10 \mathrm{~Hz}$} & $1 \mathrm{~Hz}$ \\
\hline
\end{tabular}

are between $10^{4}$ (low contrast) to $10^{10}$ (high contrast). A low contrast ratio can cause pre-ionization of the target before the arrival of the main pulse, thus disrupting the laser-target interaction mechanism. In the case of sufficient thick target, this phenomenon can improve the ion acceleration due to stronger main pulse absorption, while in the case of nanometer thick target, the low pulse contrast can destroyed the target before the arrival of the main pulse, thus requiring very high contrast. The

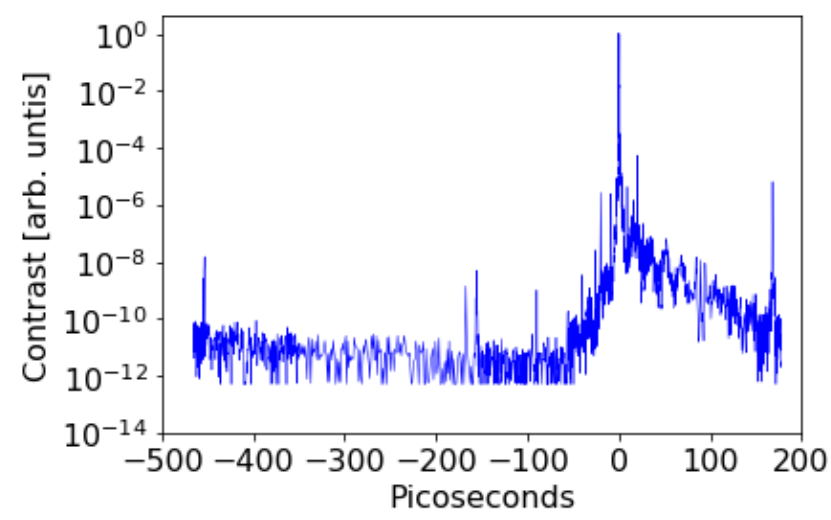

Figure 5.2: VEGA-3 contrast laser pulse.

framework of this thesis concerns VEGA-3 laser system. The contrast has been measured with a Third order autocorrelator (Sequoia by Amplitude Technologies) and was find to be up to $\approx 5.10^{12}: 1$ pulse contrast. (see Figure 5.2 and Table 5.2).

Table 5.2: VEGA-3 laser contrast ratio for different delay time.

\begin{tabular}{l|l}
\hline \hline 1 picosecond & $2.10^{-5}: 1$ \\
\hline 5 picoseconds & $<10^{-8}: 1$ \\
\hline 10 picoseconds & $8.10^{-9}: 1$ \\
\hline$>100$ picoseconds & $5.10^{-12}: 1$ \\
\hline
\end{tabular}




\section{$5.2 \quad$ Experimental setup}

The compressed p-polarized laser pulse provides by VEGA-3 was focused onto micrometer-thick aluminum solid target with a $10^{\circ}$ incidence angle with respect to the normal of the target. The experimental setup was set in a dedicated vacuum chamber evacuated to about $10^{6}-10^{5}$ mbar. Figure 5.3 represents the main elements

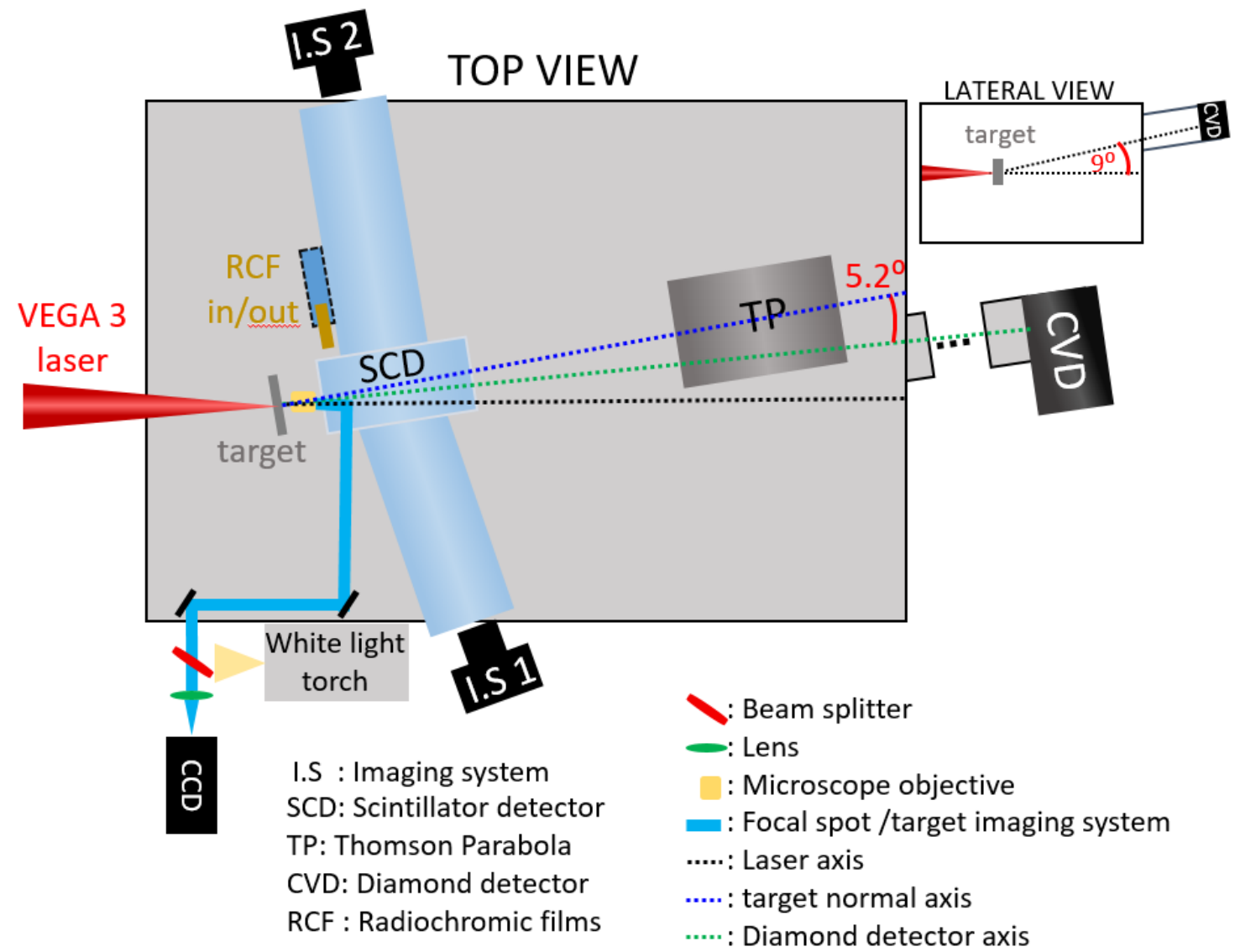

Figure 5.3: Experimental setup for laser-driven proton acceleration with VEGA-3 laser system.

of the experimental setup which is composed by the VEGA-3 laser beam focusing, the target, the optical imaging system for target and focal spot alignment and the particle diagnostics.

\subsubsection{Target and focal spot alignment}

The laser pulse was focused by an OAP silver coated with $15^{\circ}$ off-axis angle and $250 \mathrm{~cm}$ focal length. The focal spot was estimated and averaged on 20 acquisitions made throughout the experimental campaign, and was estimated about $12 \mu \mathrm{m} \pm 1$ 
$\mu \mathrm{m}$ diameter at FWHM. One of the 20 acquisitions is shown in Figure 5.4. The targets

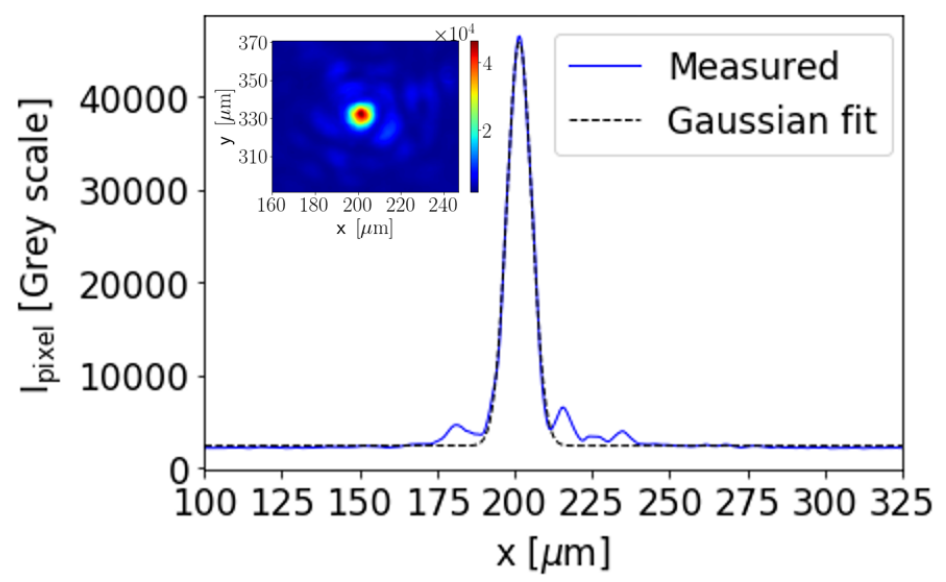

Figure 5.4: Picture and transverse cut of the focal spot for one of the 20 acquisitions made throughout the experimental campaign. Enlarged CCD output image of the focal spot (rainbow scale inset). The focal spot diameter at FWHM was estimated about $10.5 \mu \mathrm{m}$ along $\mathrm{x}$-axis and $12 \mu \mathrm{m}$ along $\mathrm{y}$-axis.

were placed on a support in a free position using epoxy-type glue. The holder has a capacity of 100 shots, and each hole has a diameter of $800 \mu \mathrm{m}$ and separated from each other by $3.5 \mathrm{~mm}$ (see Figure 5.5). The laser-target interaction point occurs at

a)

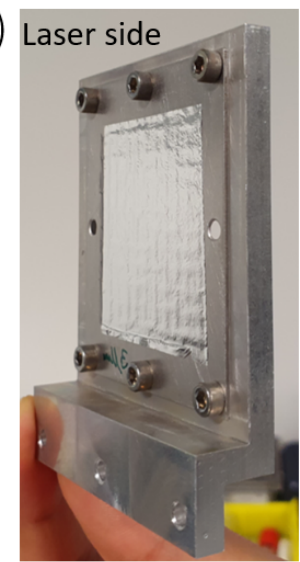

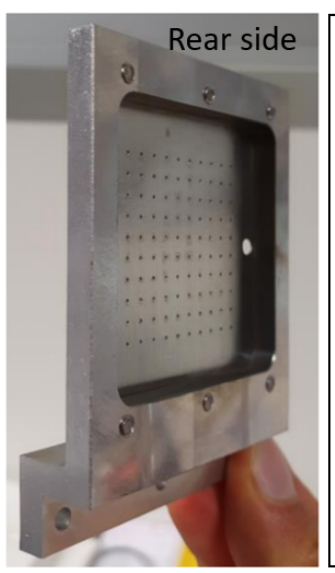

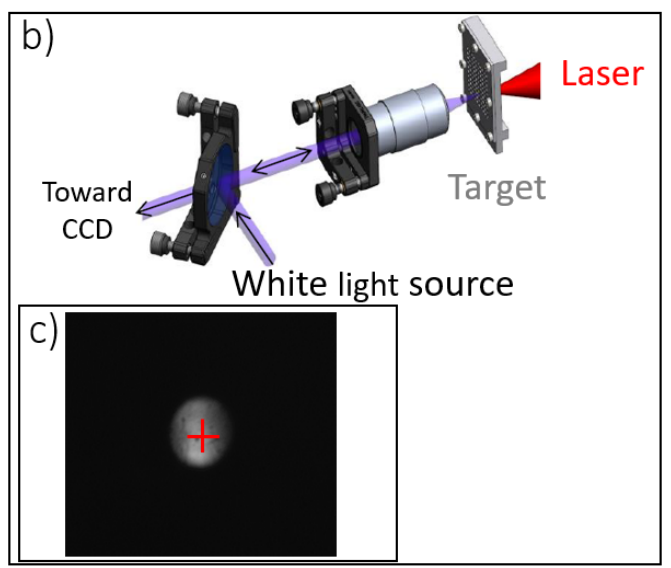

Figure 5.5: a) Free standing aluminum target. b) Illustration of the target alignment method. A white light source is sent from the rear side of the objective using a beam splitter where it is then focused on the rear side of the target. The light reflected from the target returns through the objective and part of the light is transmitted through the beam splitter and goes to the CCD. c) CCD output image of the back side of aluminum target illuminated with a white light source. 
the Target Chamber Center (TCC) which is defined by a $10 \mu \mathrm{m}$ diameter gold coated TIP which can be displaced in and out thanks to calibrated motorized translation stages. Target and focal spot are aligned with the same imaging system (we will call it focal spot (FP) imaging system for simplicity). For daily basis alignment, the TIP is first moved at TCC in order to check whether the FP imaging system is still focusing it properly. The TIP is then moved out and TCC position reference is now set by the FP imaging system, therefore marking the TCC reference point on the CCD. Laser focal spot can then be placed at TCC using FP imaging system reference. For target alignment, a white light source illuminates the target rear surface thanks to a beam splitter set outside the interaction chamber, on the optical path of the FP imaging system (see Figure 5.5). The white light is sent on the beam splitter to co-propagate along the optical path of the FP imaging system in the opposite direction towards the rear side of the microscope objective from which it is focused onto the rear side of the target. The light reflected by the target surface is then returned along the optical path and the light transmitted by the beam splitter reaches the CCD. The target is then aligned in lateral thanks to the TCC references of the CCD. Motorized stages allow for target positioning in all three spatial directions with a resolution in the micrometer-range.

\subsubsection{Overview of the diagnostic system}

\section{Scintillator detector}

For this experiment, the scintillator detector was only composed by the central metallic box of the detector built for the experiment made at the HZDR facility, placed at $2.4 \mathrm{~cm}$ downstream from the target. The detector was composed by ten square scintillator layers BC-400 of $54 \mathrm{~mm} \times 54 \mathrm{~mm}$, each of them separated by a relative angle of $25^{\circ}$. The first seven layers had a nominal thickness of $100 \mu \mathrm{m}$ and the last three $250 \mu \mathrm{m}$ in order to compensate the signal reduction inversely proportional to the square of the distance from the source and the lower particle number at high energy. Each layer was preceded by a lexan-type opaque material with a fixed thickness of a few microns, in order to isolate the signals emitted by each layer (Figure 5.6). A central hole has been made in each layer of lexan and BC-400 with the intention of letting the high energy protons propagate to the TP located $52 \mathrm{~cm}$ farther on the proton axis. The scintillation emission was collected from the rear face of the scintillators by two imaging systems, one for the odd layers (imaging system 1) and another for the even layers (imaging system 2). Each imaging system consisted of a Point Gray Blackfly monochrome model CCD camera and a NIKON AF-S DX NIKKOR 18-105mm $\mathrm{m} / 3.5-5.6 \mathrm{G}$ ED VR lens placed outside the chamber. The detector was surrounded by two aluminum tubes on each side to isolate the signal from background noise. Position of the CCD 1 with respect to the first and last layer was 91.5 and $89.5 \mathrm{~cm}$, respectively. Position of the CCD 2 with respect to the first and last layer was 100 and $98 \mathrm{~cm}$, respectively. The composition of the detector was made in such a way that it could detect proton energies from $860 \mathrm{keV}$ up to $10.7 \mathrm{MeV}$. The vertical 
spatial resolution of the detector was estimated to 112 and $94 \mu \mathrm{m}$ for I.S 1 and I.S 2, respectively. The horizontal resolution was lowered by a factor 3.2, thus leading to 360 and $300 \mu \mathrm{m}$ for I.S 1 and I.S 2, respectively. The detector was placed on a linear motorized stage in order to be moved out when other diagnostics were inserted close to the rear side of the target.

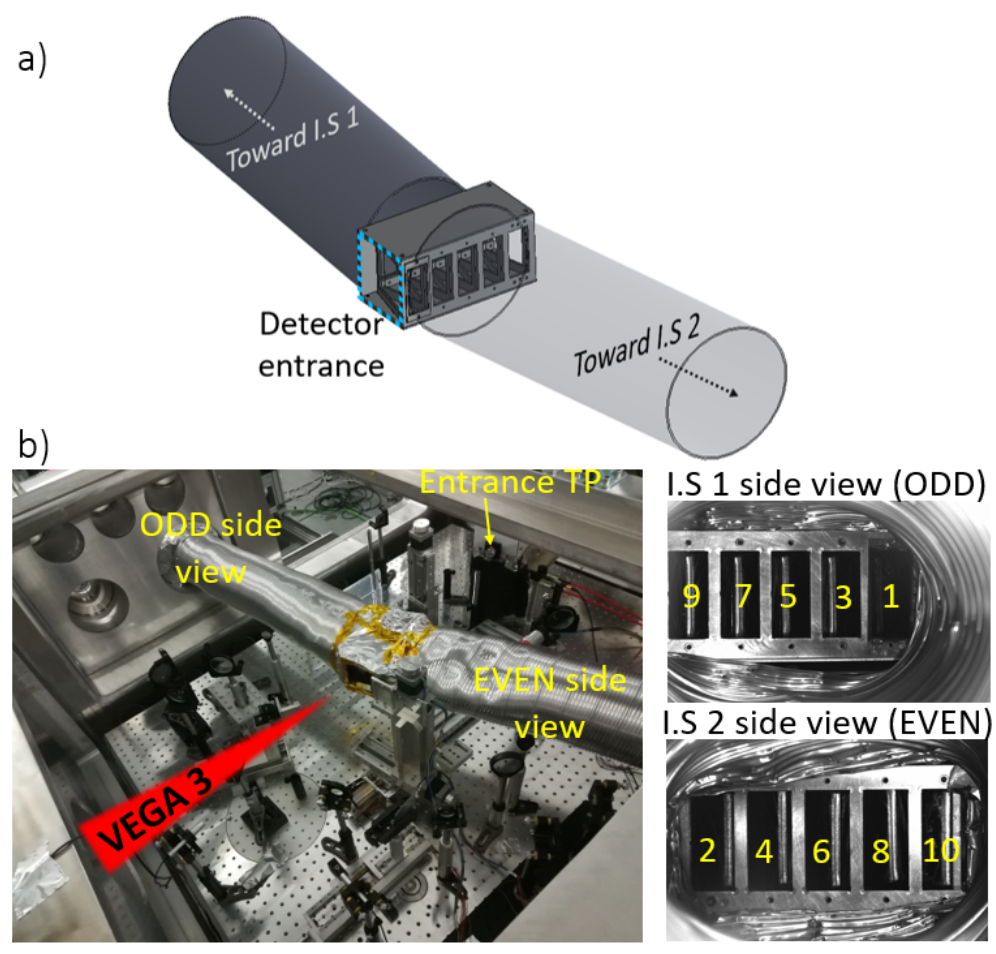

Figure 5.6: a) Scintillator detector design. b)Top view of the scintillator detector in the interaction chamber (right) and imaging system view for both odd and even sides (left).

\section{Other particle diagnostics}

Additional diagnostics were used to cross check the results obtained with the scintillator detector. The energy-resolved spatial distribution of the accelerated proton beam was measured with stacks of RCF (Gafchromic HDV2) when the scintillator detector was moved out. The stack was placed at $3 \mathrm{~cm}$ downstream from the target and was composed by 13 HDV2 Gafchromic layers (see section 3.5.3) alternating with aluminum shielding layers in order to resolved proton energies up to $24.8 \mathrm{MeV}$. Energy range resolution for each layer varying between 2.2 and $1.1 \mathrm{MeV}$. A Thomson parabola spectrometer (TP) was placed at $52 \mathrm{~cm}$ downstream from the target, along the target normal axis. Signal was recorded using (MCP) detectors coupled with fluorescent screens and were read out with CCD cameras. In addition, a Polycrystalline CVD diamond detector was used for Time of Flight (TOF) measurements. It was 
placed at $188 \mathrm{~cm}$ downstream from the target with a relative angle with respect to the normal target axis of $5.2^{\circ}$ and $9^{\circ}$ along the lateral and vertical axis, respectively (see Figure 5.3).

\subsection{Experimental results}

The general purpose of this experimental campaign was to study the performance of laser-driven proton acceleration with VEGA-3 laser system in the TNSA regime and compare it to established results from other laboratories with similar laser system. A large amount of data have been acquired during the experimental campaign with the intention of developing a Machine Learning method to study VEGA according to different parameters such as target thickness, Pulse duration or laser energy. The goal of this preliminary study was to get the basic characterization of laser-driven ion acceleration such as proton cut-off, divergence and spectrum distribution. In this chapter, part of the experimental results are shown together with a preliminary evaluation and comparison with existing scaling laws.

The experiment was performed by scanning different pulse durations in the range of (30-500 fs) for fixed laser energies. Here we report one example where VEGA-3 was focused on a $6 \mu \mathrm{m}$ thick aluminum target with a $10^{\circ}$ incidence angle with respect to the normal of the target and an energy $E_{L}$ of $10 \mathrm{~J}$ (on target) corresponding to maximum intensity of $\approx 3 \times 10^{20} \mathrm{~W} . \mathrm{cm}^{-2}$ (about $70 \%$ of the laser energy within the focal spot) with a temporal intensity contrast conditions presented above.

\section{Proton energy scaling}

Results for the maximum proton energy as a function of the laser pulse duration are summarized in Figure 5.7. The pulse duration $\tau_{L}$ was scanned over of wide range, from $30 \mathrm{fs}$ up to $500 \mathrm{fs}$ and data were simultaneously recorded using a TP and CVD diamond detector. The proton cut-off energy is in agreement for both diagnostics, with a maximum proton energy close to $14 \mathrm{MeV}$ and obtained for pulse compression around 200 fs. However, a low stability of the proton cut-off energy is observed when repeating a shot with the same laser parameters and one of the reasons could be the uncontrollable target surface inhomogeneities and/or the shot-to-shot laser pointing instability. The general profile behavior of the protons cut-off energy seems to follow the prediction of the scaling model by Schreiber et al [Schreiber et al., 2006]. It shows the presence of an optimum pulse duration close to $200 \mathrm{fs}$. The decrease of the proton cut-off energy for shorter pulse duration can be explained by the reduction of the interaction time which, in consequence, influences the acceleration efficiency. Experimental data have been compared with the electrostatic scaling model from Schreiber et al (see Figure 5.7). We considered $70 \%$ (7 J) of the laser energy concentrated inside the focal spot and the laser efficiency dependent on the laser intensity according to equation (3.72). In order to match the profile behavior of our experimental data with the analytic model, the focal spot radius has been set as $r_{L}=9 \mu \mathrm{m}$ (considering that the focal spot dimension has been estimated at low power, this parameter is not 
measured and consequently uncertain when shotting at high energy laser) and the half angle electron divergence $\theta=30^{\circ}$, in agreement with the range of the accessible data in the literature [Adam et al., 2006; Fuchs et al., 2003; Stephens et al., 2004]. As a first sight, this preliminary analysis shows experimental data behavior in agreement with the model. However, the electron divergence is a complex mechanism [Debayle et al., 2010] depending on several processes such as preplasma or laser intensity, thus making difficult its estimation due to the lack of reliable models. In addition, the low-intensity wings present in the spatial distribution of the focal spot profile contain part of the incident laser energy and may reduce the laser efficiency.

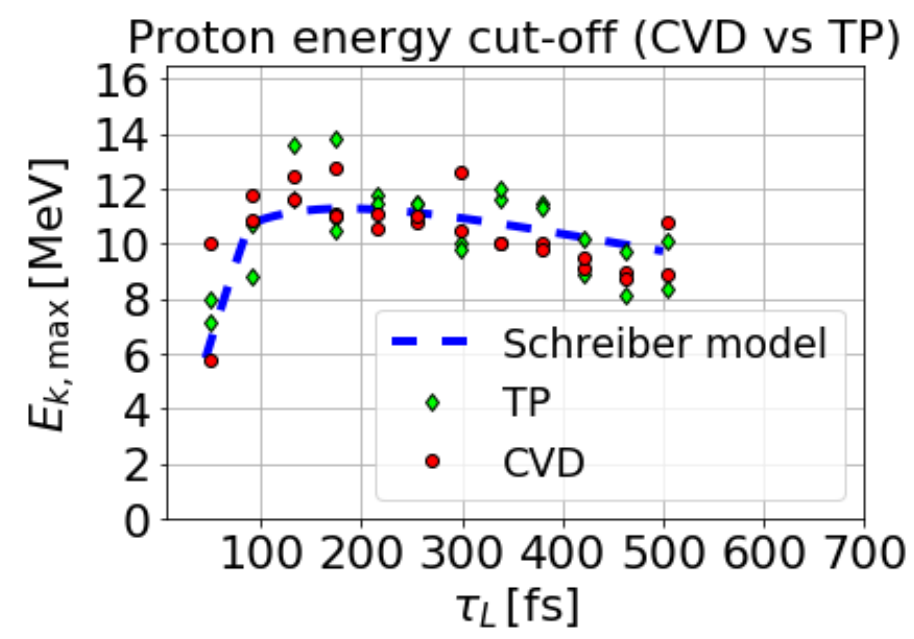

Figure 5.7: Laser pulse dependence of maximum proton energies for TP (green lozenges) and CVD diamond detector (red circles). The blue dashed line corresponds to Schreiber model for: focal spot radius $r_{L}=9 \mu \mathrm{m}$, target thickness $d=6 \mu \mathrm{m}$, laser energy $E_{L}=10 \mathrm{~J}$ (70\% within the focal spot) and electron half-divergence $\theta=30^{\circ}$.

\section{Proton spectrum and beam divergence}

Simultaneous shots with half-RCF geometry and TP were performed and are presented in Figure 5.8. The proton cut-off energy is in agreement for both diagnostics. A slight increase can be observed for the RCF which come from a possible misplacement of the diagnostics regarding the central axis of proton propagation. The spatially integrated proton spectrum shows a typical TNSA-like spectrum and an increase of the proton number per energy bin $d N / d E$ with decreasing proton energy. The RCF diagnostic has been calibrated with a well known proton source and gives an estimation of the total number of protons per shot about $\sim 10^{9}$. TP detector is at the moment not absolutely calibrated and can only give information about the proton energy range.

Figure 5.9 shows a proton beam profile recorded with the RCF diagnostic for a maximum energy of $13.1 \mathrm{MeV}$ (lower limit for the maximum proton energy). The diver- 
a)

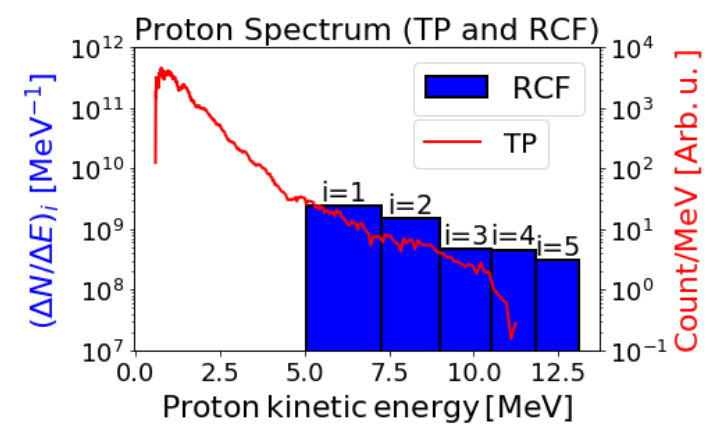

b)

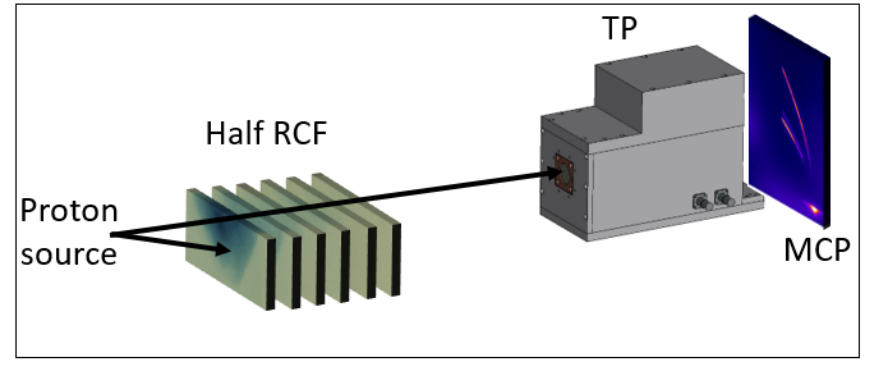

Figure 5.8: a) Proton spectrum distribution for TP and scintillator detector. b) Illustration of the set-up for simultaneous data acquisition.

gence was evaluated up to $40^{\circ}$ for the lowest proton energies and decreases for higher proton kinetic energy, in agreement with the characteristics of laser-driven ions generally reported [Cowan et al., 2004]. One can observe a very small angular deflection of the proton distribution from the expected target normal direction toward the laser propagation axis. One hypothesis for this behavior may be the target rear surface pre-heating. Some studies have shown that the ASE pedestal of the laser pulse may initiate a shock wave at the target front surface, which may break through the target rear surface and generate a deformation [Lundh et al., 2007]. 

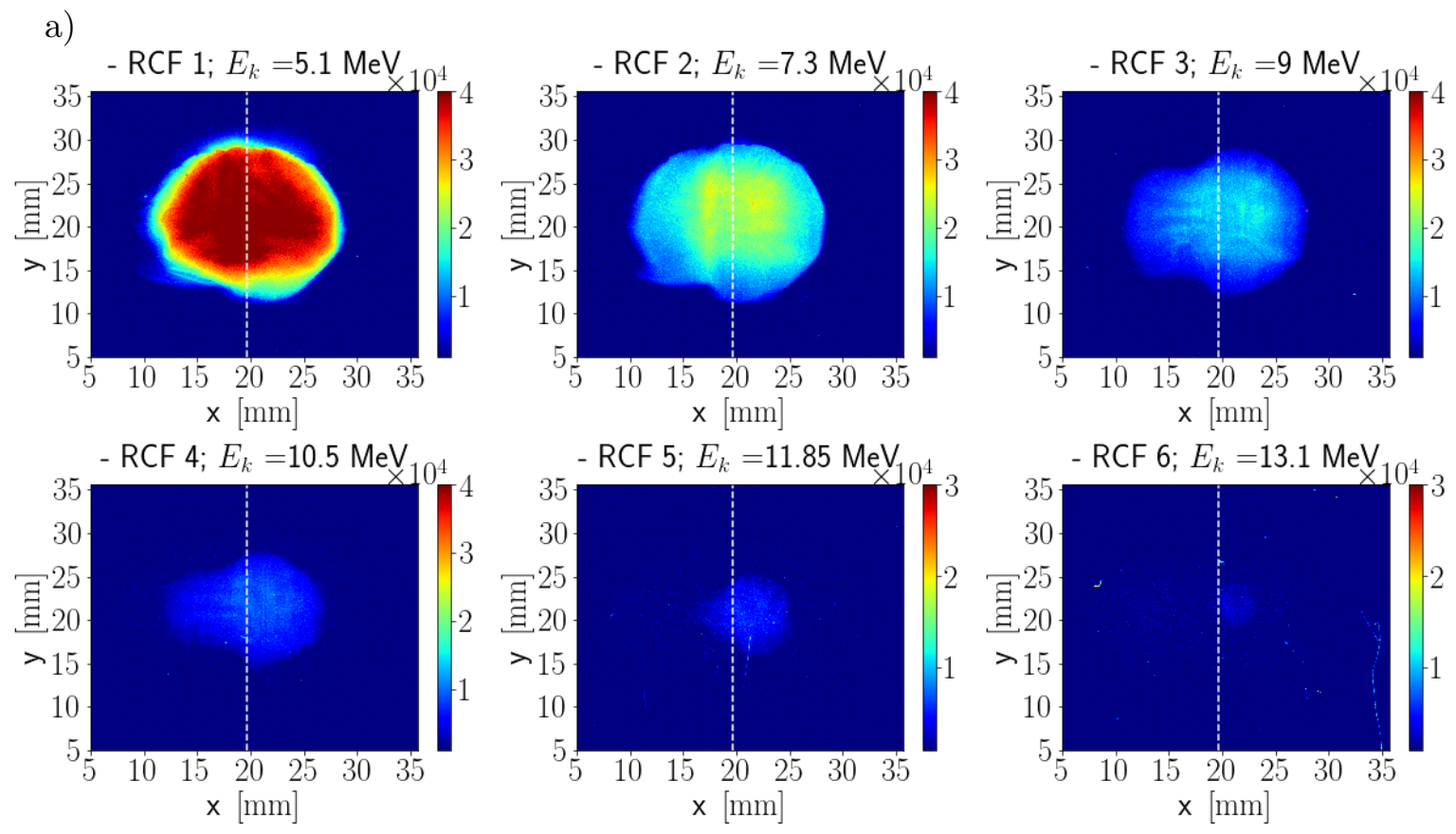

b)
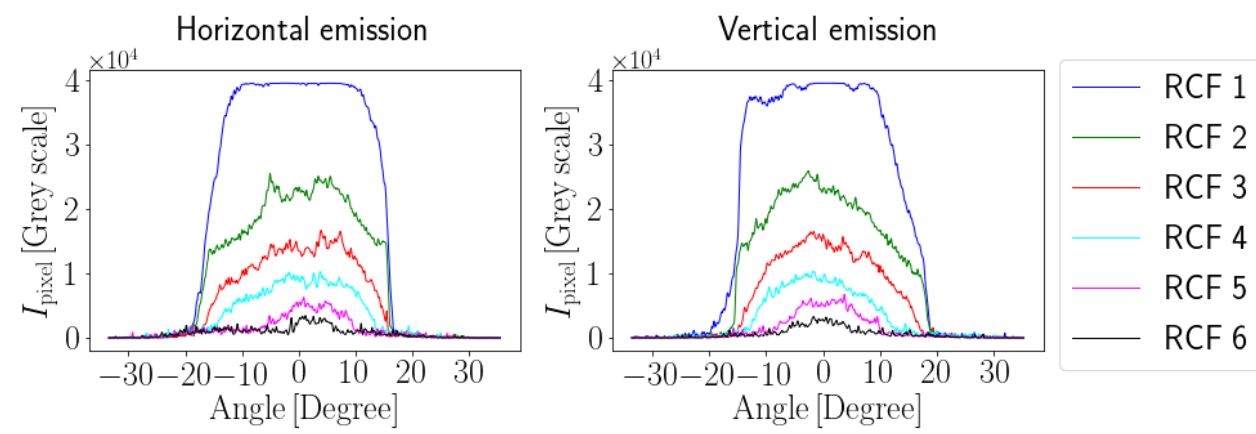

Figure 5.9: Proton emission distribution from RCF stack resulting from the interaction of VEGA-3 laser of energy $E_{L}=10 \mathrm{~J}$ with an aluminum target of $6 \mu \mathrm{m}$ thick. Each RCF layer represents protons with energies at or above the respective kinetic energy $E_{k}$. a) Proton beam profile from the scanned RCF layers (Grey scale). b) Estimation of the divergence for the horizontal and vertical emission of the proton beam. The vertical white dashed lines in a) indicates the target normal axis. 


\section{Comparison with the scintillator detector}

Data recorded with the scintillator seems to underestimate the proton cut-off energy. Several shots recorded by the TP and the scintillator detector (not simultaneously) have shown a difference in energy between the two detectors up to $3 \mathrm{MeV}$, with energy always lower for the detector scintillator. As reported in the previous section, the last layers receive a small number of protons per area due to the signal intensity reduction that scales as $\propto 1 / r^{2}$ with the distance. This affects the resolution in the high energy tail of the exponential proton spectrum. Figure 5.10 shows the spatially resolved proton spectrum estimated with the scintillator detector for a proton cut-off of $5.1 \mathrm{MeV}$. Although the shot is not the same than for the RCF spectrum presented in Figure 5.8, with a different proton cut-off energy, we can observe a consistency in the quantity of proton obtained. Figure 5.11 shows the proton beam

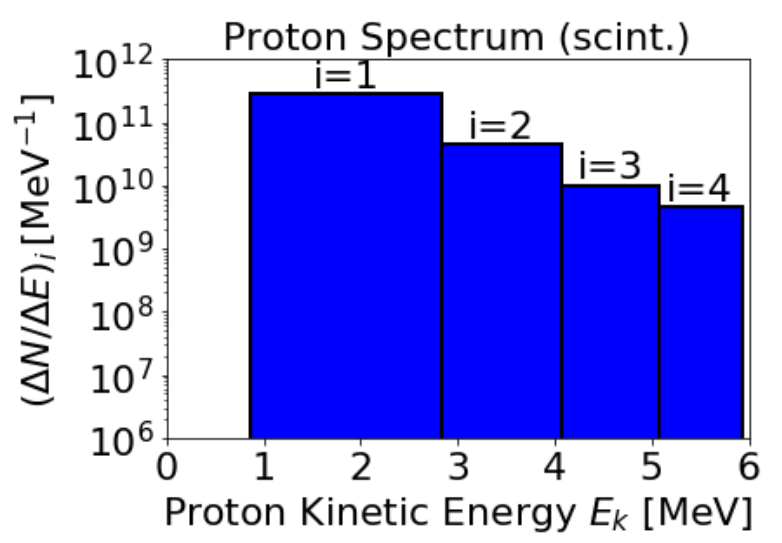

Figure 5.10: a) Spatially integrated proton spectrum for the scintillator diagnostic.

divergence reconstruction from initial CCD output image. A super-Gaussian function with $f_{x}^{f i t}=A \exp \left(\frac{\left(x-x_{0}\right)^{2}}{2 . \sigma_{x}^{2}}\right)^{P}$ was fitted along the horizontal (x) and vertical (y) axis of the initial signal. The resulting obtained function was then applied to built the Gaussian-like signal distribution. Using our model for multiple scattering correction (see Section 4.4.4), the initial beam divergence was estimated and is represented in Figure 5.11(c).

\subsection{Conclusion}

This experiment aimed to promote the VEGA-3 laser system performance, where the capability of generating a stable and controlled proton source driven by laser at HRR for application has been established. We successfully demonstrated the online characterization of the laser driven proton source through the implementation of multiple diagnostics coupled with calibrated RCF diagnostic. Comparison of the 
experimental data with literature and scaling model showed low efficiency of the maximum energy of the accelerated proton beam with VEGA-3. The presence of an ASE pedestal affecting the proton acceleration performance at higher intensity can be one of the possible reasons. Indeed, the maximum proton energy is strongly correlated with the plasma conditions at the target surfaces which can be deteriorated by prolongation of the ASE pedestal [Kaluza et al., 2004]. Therefore, future improvement may be done concerning the laser contrast and its stability to optimize and raise up the proton energy. In addition, we have shown the successful implementation of the scintillator detector as a complementary diagnostic for the proton beam reconstruction profile. Results from the detector are consistent with the other diagnostics even if a general underestimation in the determination of the proton maximum energy is observed with the scintillator detector due to a lack of signal resolution in the last layers of the detector and will have to be improved for the future experiments, as discussed in previous chapter. 
a)
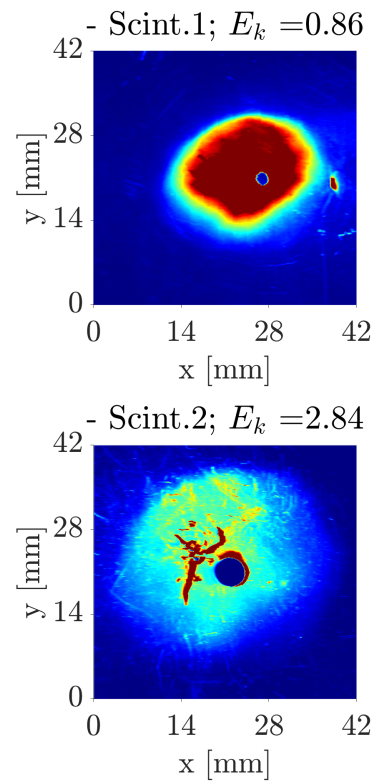

- Scint. $3 ; E_{k}=4.07$
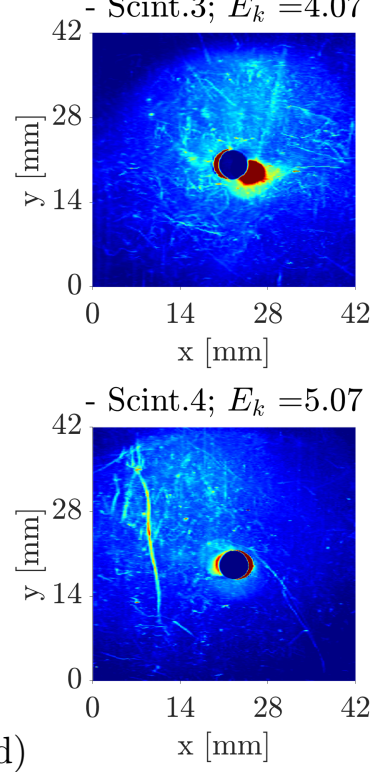

b)
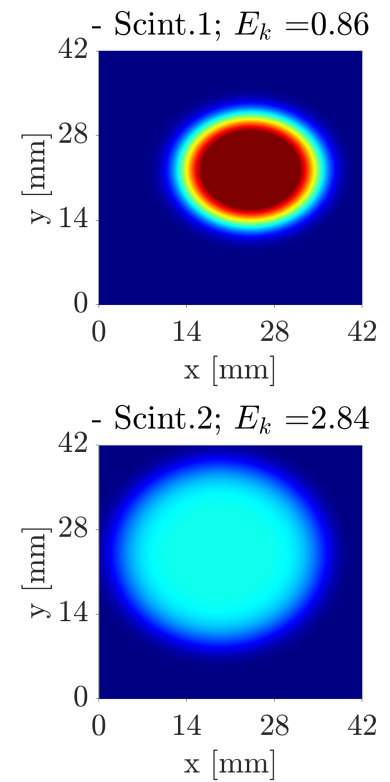

- Scint. $3 ; E_{k}=4.07$
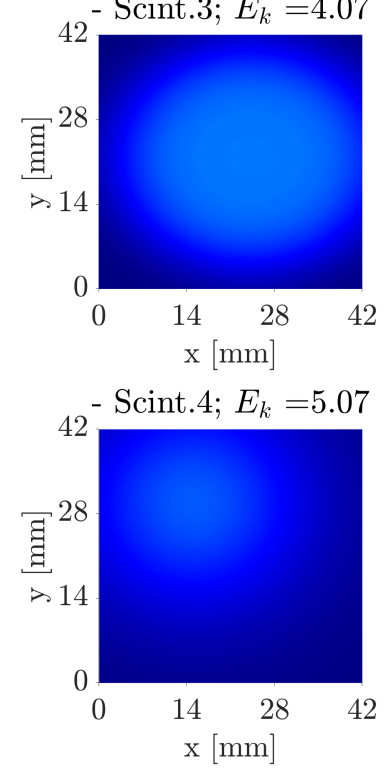

c)
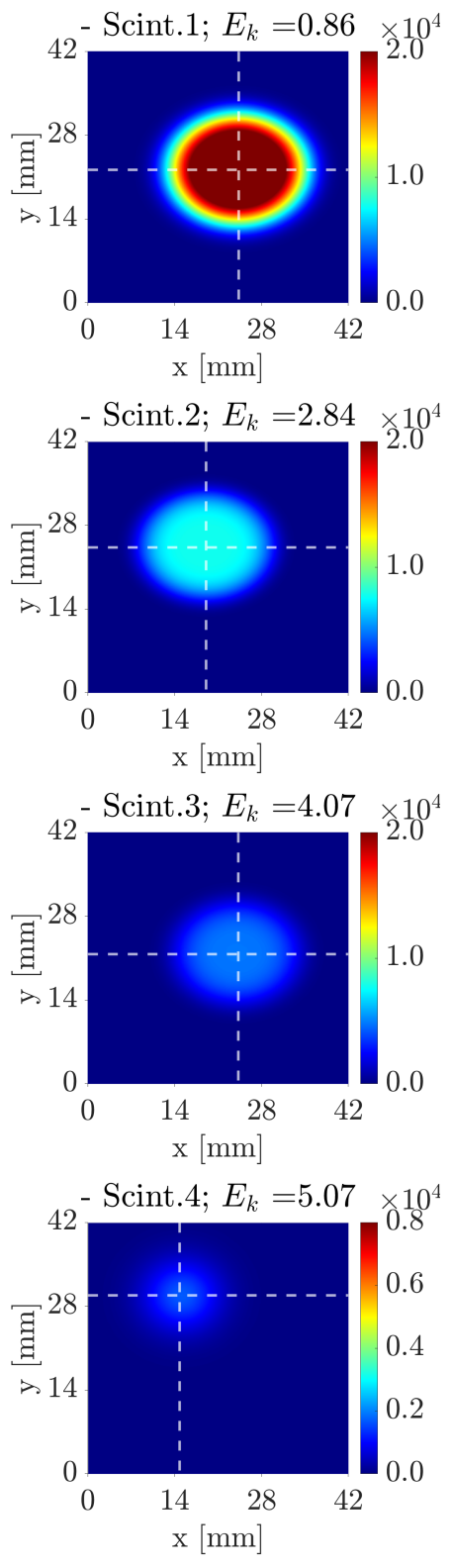
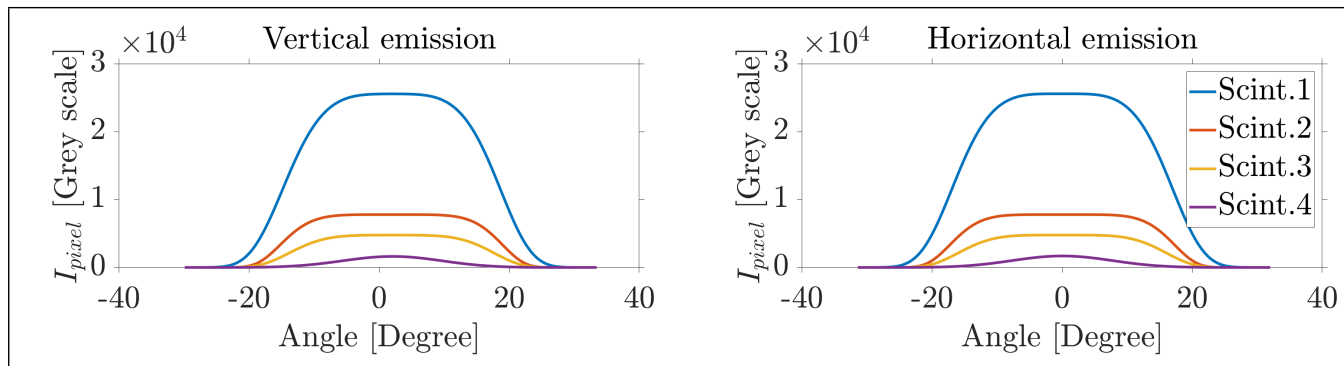

Figure 5.11: Proton emission distribution from scintillator detector. a) Reconstructed geometry of CCD output image in grey scale. b) Super-Gaussian fit of images represented in a); c) Super-Gaussian fit representing the reconstructed initial proton beam divergence. White dashed lines in c) represent the location of the transverse cuts for the divergence representation in d). Each scintillator layer represents protons with energies at or above the respective kinetic energy $E_{k}$. 


\section{Chapter 6}

\section{Conclusions and Perspectives}

The work presented in this thesis has been dedicated to the study of the generation, transport and optimization of proton sources driven by ultra-short (fs) and ultra-intense $\left(>10^{18} \mathrm{~W} . \mathrm{cm}^{-2}\right)$ laser pulses working at HRR with the aim to use such sources for several applications. The main result presented was the characterization of proton sources through the development of dedicated 2D scintillator based spatialenergy on-line detector finally successfully implemented in an experimental campaign at the CLPU in Salamanca, Spain.

This innovative detector showed the possibility to reproduce the performance of conventional passive detectors such as RCF at HRR, thus paving the way for a new class of online detectors in laser-plasma physic experiments. The characterization of the detector with a conventional proton source at the tandem accelerator from the CMAM demonstrated that the proton spatial distribution can be successfully imaged by our detector. The scintillator has shown a response in agreement with the literature: i) a linear response for proton flux up to $10^{11}$ protons. $\mathrm{cm}^{-2} \cdot \mathrm{s}^{-1}$ ii) a strong attenuation of the response to deposited energy at high stopping power due to quenching effect and appears for specific deposited energy higher than $30 \mathrm{MeV} / \mathrm{cm}$. Although the first steps of calibration to be able to use it as a $2 \mathrm{D}$ scintillator based proton detector were performed, the next step would be to characterize the scintillator response with a proton energy covering a wider range, in order to explore a response outside the quenching regime.

On the other side, the capabilities of our detector under extreme conditions of laserplasma interaction have been tested. For this, the detector was implemented in real experimental conditions in which laser driven proton sources have been generated. As a proof of principle, the detector has been successfully tested in a laser-based acceleration experiment with capability of being set at HRR, giving a direct feedback about the $2 \mathrm{D}$ spatial and energy resolution of the proton beam. However, uncertainties on the scintillator thickness up to $30 \%$ have been observed and care must be taken when interpreting the detector response for the proton spectrum reconstruction. In the future, the development of a metrology method must be set in order to characterize 
the surface roughness and thickness of the scintillator layer. Although adjustments are necessary in order to be able to use it as an absolute measurement diagnostic (background correction, compactness of the detector, viewing system...), the detailed analysis reported in this study have demonstrated promising capabilities of such a diagnostic.

A proton source driven by ultra-short and ultra-intense laser pulses working at HRR has been established with VEGA-3 laser. The preliminary results presented in this thesis were related to the study of VEGA-3 performance on solid target (target of 6 $\mu \mathrm{m}$ thick $\mathrm{Al}$, laser energy $E_{L}=10 \mathrm{~J}$ ) for pulse durations ranging from 30 fs to 500 fs. The profile behavior of the protons maximum energy according to pulse duration seems to follow the prediction of scaling model from Schreiber at al., with the existence of an optimal pulse duration close to $200 \mathrm{fs}$ ( $14 \mathrm{MeV}$ maximum proton energy) corresponding to a laser intensity of around $I_{L}=4 \times 10^{19} \mathrm{~W} \cdot \mathrm{cm}^{-2}$. Simultaneous shots done with radiochromic films and Thomson parabola spectrometer showed a concordance in the proton cut-off energy. In addition, the new developed detector has been implemented in the experimental set-up to test its response with respect to other diagnostics. The scintillator detector response has shown consistency on the proton cut-off energy behavior with respect to other diagnostics, even if it appears that the detected proton energy is always lower for the scintillator detector. The main reason of such underestimation is coming from its dimension that is several times bigger than the conventional RCF stack. The last layers receive a small number of protons per area due to the signal intensity reduction that scales as $\propto 1 / r^{2}$ with the distance. This affects the resolution in the high energy tail of the exponential proton spectrum.

Finally, this was the first experiment where on-line measurements and on-line analysis where simultaneous done at CLPU. However, targetry systems need further development to match the VEGA repetition rate potential. The target movement and alignment still need to be enhanced for working modes above $0.1 \mathrm{~Hz}$; on the other side the present proton diagnostics for the HRR acquisition have shown to be perfectly prepared and calibrated to substitute in performance the conventional passive detectors. 


\section{Chapter 7}

\section{Resumen y conclusiones de la tesis}

El trabajo presentado en esta tesis consiste en el estudio de la generación, transporte y optimizació de fuentes de protones generadas por pulsos de láser ultracorto (fs) y ultra intensos $\left(>10^{18}\right.$ W.cm $\left.{ }^{-2}\right)$ que pueden trabajar a alta tasa de repetición con el objetivo de utilizar dichas fuentes para aplicaciones civiles. El resultado principal presentado es la caracterización de dichas fuentes de protones mediante el desarrollo de un detector a tiempo real de energía espacial basado en centelleo 2D, finalmente implementado con éxito en una campaña experimental en el Centro de Láseres Pulsados (CLPU) en Salamanca, España.

La primera parte de la tesis se ha centrado en la descripción de la teoría de los protones y los electrones rápidos generados por la interacción del pulso láser ultra intenso en el plasma sobredenso. Los principales mecanismos de calentamiento de electrones y su transporte en un blanco sólido se han presentado primero para luego centrarse en los haces de protones generados por láser generados por Target Normal Sheath Acceleration (TNSA) y su transporte en la materia.

La segunda parte de la tesis se dedicó al desarrollo del primer detector de protones basado en centelleo 2D capaz de ser usado a alta repetición. Este detector innovador mostró la posibilidad de reproducir el rendimiento de los detectores pasivos convencionales tal como RCF en alta repetición, allanando así el camino para una nueva clase de detectores a tiempo real en experimentos físicos de láser-plasma. El detector está compuesto por centelleadores rápidos de plástico BC-400, colocados con un ángulo relativo entre ellos, lo que permite medir tanto la energía del haz de protones como su distribución angular. El detector ha sido diseñado y construido en el CLPU y probado en colaboración con instalaciones de toda la UE. Por un lado, el detector se ha caracterizado con una fuente de protones convencional en el acelerador tándem del Centro de Micro-An álisis de Materiales de Madrid (CMAM) con el fin de demostrar que la distribución espacial de protones puede ser captado con éxito por nuestro de- 
tector. El centelleador ha demostrado una respuesta de acuerdo con la literatura: i) una respuesta lineal para el flujo de protones hasta $10^{11}$ protones. $\mathrm{cm}^{-2} \cdot \mathrm{s}^{-1}$ ii) una fuerte atenuación de la respuesta a la energía depositada a una alta potencia de frenado debido al efecto de extinción que aparece para la energía depositada específica superior a $30 \mathrm{MeV} / \mathrm{cm}$. Por otro lado, se han probado las capacidades de nuestro detector en condiciones extremas de interacción láser-plasma. Para ello, el detector se implementó en condiciones experimentales reales en las que se han generado fuentes de protones generadas por láser. El detector se probó con éxito en un experimento de aceleración láser con la ventaja de usarse a alta repetición, lo que proporciona una retroalimentación directa sobre la resolución espacial y de energía 2D del haz de protones. Sin embargo, se han observado incertidumbres en el espesor del centelleador de hasta un $30 \%$ y se debe tener cuidado al interpretar la respuesta del detector para la reconstrucción del espectro de protones. En el futuro, se debe establecer el desarrollo de un método de metrología para caracterizar la rugosidad superficial y el espesor de la capa de centelleo. Aunque se necessitan ajustes para poder utilizarlo como un diagnóstico de medición absoluto (corrección de fondo, compacidad del detector, sistema de visualización ...), el análisis detallado reportado en este estudio ha demostrado capacidades prometedoras de dicho diagnóstico.

La última parte de la tesis se refiere al establecimiento de una fuente de protones generada por pulsos de láser ultracortos y ultra intensos que trabajan en alta repetición con el láser VEGA-3. Los resultados preliminares presentados en esta tesis están relacionados con el estudio del rendimiento de VEGA-3 en un blanco sólido (blanco de $6 \mu \mathrm{m}$ de $\mathrm{Al}$ de espesor, energía láser $E_{L}=10 \mathrm{~J}$ ) para duraciones de pulso que van desde $30 \mathrm{fs}$ a $500 \mathrm{fs}$. El comportamiento de la curva de la energía máxima de los protones según la duración del pulso parece seguir la predicción del modelo de escala con la existencia de una duración óptima del pulso cercana a 200 fs (energía protónica máxima de $14 \mathrm{MeV}$ ) correspondiente a una intensidad del láser de alrededor de $I_{L}=4 \times 10^{19} \mathrm{~W} . \mathrm{cm}^{-2}$. Las adquisiciones simultáneas realizadas con películas radiocrómicas y detector tipo parábola de Thomson mostraron una concordancia en el máximo de energía de los protónes. Además, el nuevo detector desarrollado se ha implementado en la configuración experimental para probar su respuesta con respecto a otros diagnósticos. La respuesta del detector de centelleo ha mostrado coherencia en el comportamiento de máximum de energía de los protones con respecto a otros diagnósticos, incluso si parece que la energía de los protones detectada es siempre menor para el detector de centelleo. La razón principal de tal subestimación proviene de su dimensión, que es varias veces más grande que la pila RCF convencional. Las últimas capas reciben una pequea cantidad de protones por área debido a la reducción de la intensidad de la señal que escala como $\propto 1 / r^{2}$ con la distancia. Esto afecta la resolución en la cola de alta energía del espectro de protones exponencial.

Por último, cabe señalar que este ha sido el primer experimento en el que se han realizado mediciones y análisis en tiempo real simultáneamente en CLPU. Sin embargo, el sistema de blancos necesita un mayor desarrollo para igualar el potencial 
de la alta tasa de repetición de VEGA. El movimiento y la alineación del blanco aún deben mejorarse para los modos de trabajo por encima de $0,1 \mathrm{~Hz}$; por otro lado, los diagnósticos de protones utilizados para la adquisición de HRR han demostrado estar perfectamente preparados y calibrados para sustituir en rendimiento a los detectores pasivos convencionales. 


\section{Appendix A}

\section{$.1 \quad$ PN and PIN junction Photodiodes}

Nowadays, $p$ - $n$ junction diodes and $p-i-n$ junctions are one of the most frequently used technology in semiconductor photodiodes, with the advantages to be both sensitive and robust, low thermal noise, fast, and easily biased. A $p$ - $n$ junction is composed by a piece of donor-doped (n-type) semiconductor material in which the charge carrier are in majority electrons and by an acceptor-doped (p-type) semiconductor material with holes as major carriers (see Figure 1a). When the two pieces are joined together, the $p$ - $n$ junction is produced and electrons from the $n$ region migrate by diffusion across this newly formed junction to fill up the holes in the $p$ region, leaving behind them the immobile positive donor ions. Similarly, holes migrate from $p$ to $n$ region, leaving negative acceptor ions behind (see Figure 1b). The immobile donor and acceptor ions creates an internal electric field around the $p$ - $n$ junction, acting as a barrier potential that prevents further migration of the carriers. As a result, the regions on either sides of the junction now becomes completely depleted of any more free majority carriers. This area around the $p-n$ junction is called the depletion layer.

The small quantity of hole in the $n$-region and electrons in the $p$-region are called minority carriers. Due to the internal electric field generated by the diffusion, holes from the $n$-region close to the $p$ - $n$ junction will be immediately drifted toward the $p$ side and electron from the $p$-region toward the $n$ side. Both processes contribute to the drift current. At zero bias, the diffusion current in the depletion region is balanced by the drift current.

In our experiment, the photodiode was used in photoconductive mode (reverse biased) as represented in Figure 2a). In this configuration, almost all the majority carriers close to the $p$ - $n$ junction are pushed away by the bias electric field, leaving behind them more immobile charged ions, and in consequence a wider depletion area. The electric field will become stronger, the drift current becomes dominant (dark current), but still is very small, on the order of microampere.

For the incident light to access, an optical window is provided with junction photodiodes. Upon illumination, i.e exposed to incident light, the drift current crossing the $p$ - $n$ junction can greatly increase. When a photon interacts within the depletion area, the electron-hole pair will be separated and the electron will drift away toward 
a)

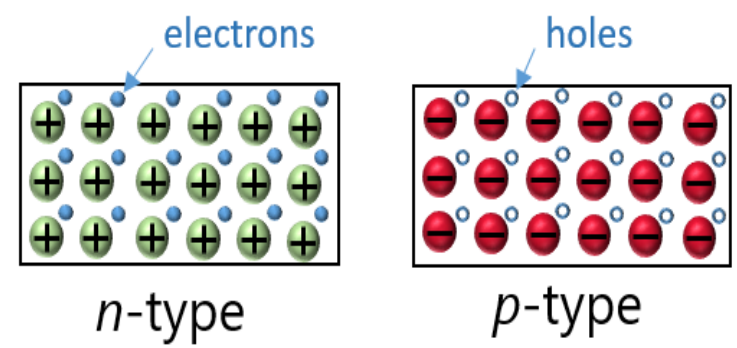

b) $p-n$ junction

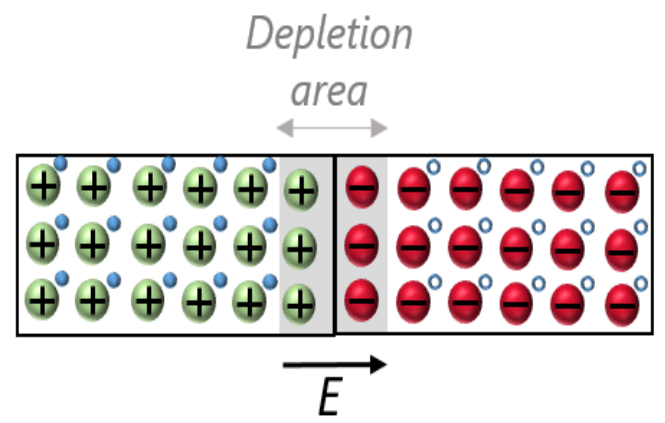

Figure 1: a) $n$-type: Donor-doped semiconductor material with electron as dominant charge carrier. $p$-type: Acceptor-doped semiconductor material. b) $p$ - $n$ junction: At zeros applied bias, an internal electric field creates a potential barrier that prevents further migration of carriers.

the $n$ side and hole toward the $p$ side, both contributing to the current flow. In a $p-n$ junction diode, only the electron-hole pairs generated with the depletion area can be influenced by the field and contribute to the external current.

By adding an intrinsec region with high resistivity between the $p$ and $n$ layers, the response time of a photodiode can be improved. Indeed, if the intrinsec layer is wide enough such that the incident light is absorbed inside it, the electron-hole pair will be generated where the electric field is strong, making the charge transport essentially due to the fast drift. This type of diode is called PIN $(p-i-n)$ diode. In a PIN diode, the depletion region is almost composed by the intrinsic region and thus much larger than in a $p-n$ junction diode and independent of the reverse bias applied. The volume where the electron-hole pairs can be generated by an incident photon is bigger and thus increases the quantum efficiency. 

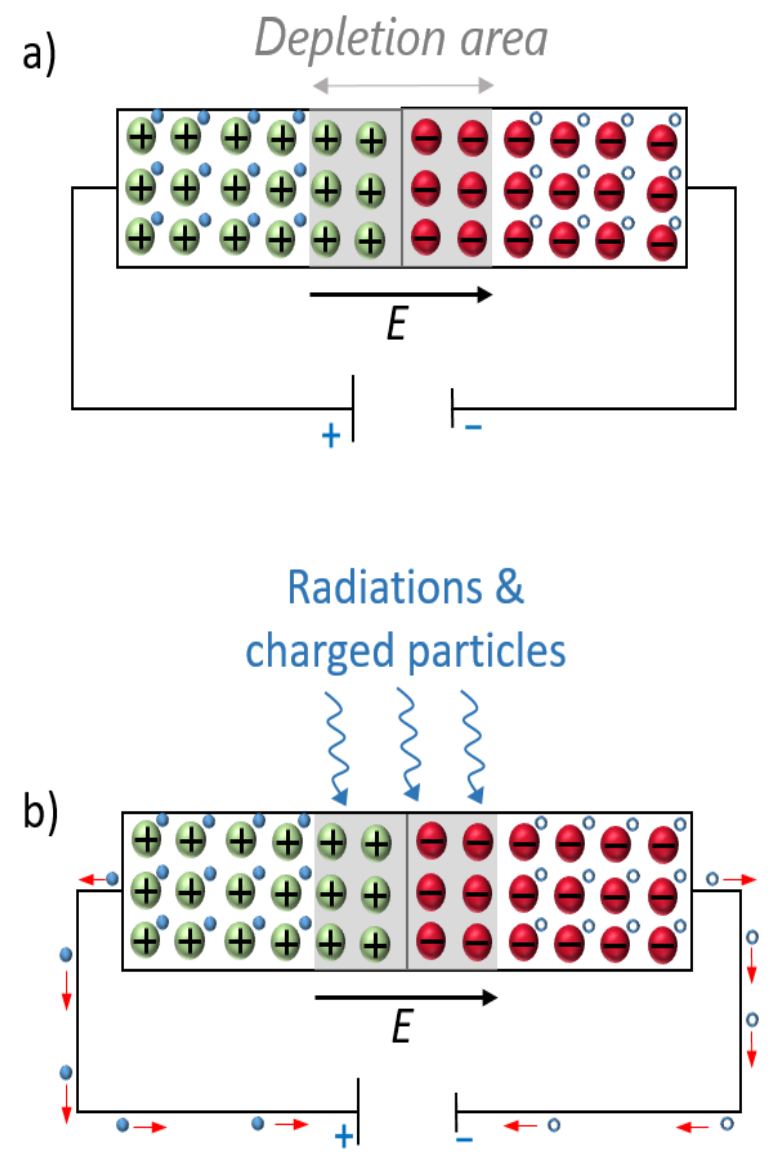

Figure 2: Under reverse bias. a) The majority charge carriers around the $p$ - $n$ junction are swept by the bias voltage and a wider depletion zone is created. b) When a photon interacts within the depletion area, an electron-hole pair is created and will be separated, the electron toward the $n$ side and the hole toward the $p$ side. 
. APPENDIX A 


\section{Appendix B}

\section{.2 Draco laser system}

The DRACO laser (the Dresden Laser Acceleration Source) is a dual beam system providing full Petawatt (30 J in $30 \mathrm{fs}$ ) and $150 \mathrm{TW}(4.5 \mathrm{~J}$ in $30 \mathrm{fs})$ after compression using CPA technique with optimized temporal pulse contrast [Obst et al., 2018] and high beam quality. The laser is based on the PULSAR laser system provided by Amplitude Technologies and uses titanium doped sapphire (Ti:Sa) crystals as gain medium at a central wavelength of $800 \mathrm{~nm}$ and a pulse a pulse bandwidth of up to $80 \mathrm{~nm}$. A schematic representation of the DRACO laser system is shown in figure 3. The laser chain starts at a Ti:Sapphire oscillator which delivers pulses at $800 \mathrm{~nm}$ central wavelength with a spectral width above $100 \mathrm{~nm}$ resulting in $10 \mathrm{fs}$ pulses at the nanojoule level. The oscillator operates at $78 \mathrm{MHz}$ repetition rate of which a Pockels cell reduces the repetition to $10 \mathrm{~Hz}$ pulse train. The pulse energy is then amplified in the booster amplifier up to microjoules level and then stretched to a duration of about 500 ps in an all-reflective stretcher module upon entering in the first CPA stage. The stretched pulses are then amplified in a regenerative amplifier to about $0.5 \mathrm{~mJ}$ for then being amplified to $25 \mathrm{~mJ}$ in a multipass amplifier. The pulse is then compressed in an air compressor to a pulse length of $30 \mathrm{fs}$ in order to optimize the temporal contrast by a cross-polarized wave (XPW) pulse cleaning. Due to the low efficiency of the non-linear cleaning process, the energy per pulse decrease to about $0.3 \mathrm{~mJ}$. These pulses are then coupled into a second CPA stage of the laser system and are amplified by successively passing through a stretcher, regenerative amplifier and a multipass amplifier up to $25 \mathrm{~mJ}$. Following, two additional multi-pass amplifiers increase the pulse energy to $100 \mathrm{~mJ}$ and then $1.5 \mathrm{~J}$. At this point, the laser is divided into 2 branches, one goes to the $40 \mathrm{~J}$ cryostat amplifier for the $\mathrm{PW}$ arm and the other to the $6 \mathrm{~J}$ cryostat amplifier for the $150 \mathrm{TW}$ arm. The two branches can be used in parallel or separatly (in this thesis only the $150 \mathrm{TW}$ arm is concerned). The last multipass amplifier is seed by a Pockels cell pulser picker to control the operation rate from single shot to continuous $10-\mathrm{Hz}$ operation mode and is cryogenically cooled to stand various repetition rate without thermal lensing. Finally, the beam is expand to its final size of $100 \mathrm{~mm}$ diameter in order to reduce the fluence on the optics and transported to the compressor to get the 30 fs pulse duration. The compression stage, the beam transport and interaction occur in vacuum to avoid possible filamentation. 
A deformable mirror is used for wavefront optimization operating in a closed loop with a sensor (PHASICS SID4) situated at the experimental cave.

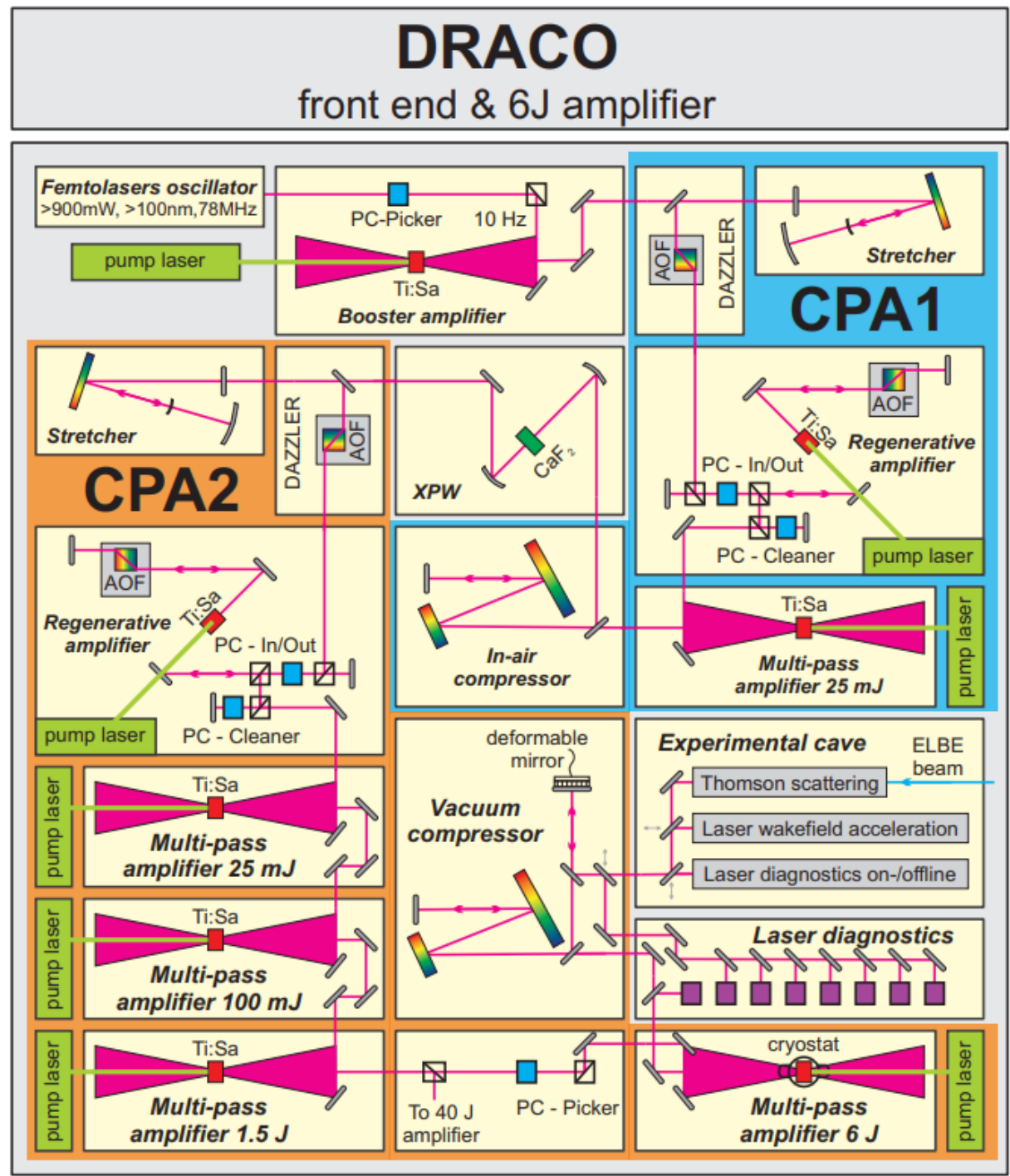

Figure 3: Setup of the DRACO laser system 150 TW arm. The used abbreviations are PC for Pockels cell. From [Couperus, 2018]. 


\section{Bibliography}

[Adam et al., 2006] Adam, J. C., Héron, A., \& Laval, G. 2006. Dispersion and Transport of Energetic Particles due to the Interaction of Intense Laser Pulses with Overdense Plasmas. Phys. Rev. Lett., 97(Nov), 205006. 123

[Aea-Tecdoc-1070, 1999] Aea-Tecdoc-1070. 1999. Techniques for High-Dose Dosimetry in Industry, Agriculture and Medicine - Proceedings of a symposium held in Vienna, 9-13 November 1998. Proceedings of a symposium held in Vienna, 25 November 1998, INIS Clearinghouse. 65

[Ageno et al., 1950] Ageno, M., Chiozzotto, M., \& Querzoli, R. 1950. Scintillations in Liquids and Solutions. Phys. Rev., 79(Aug), 720-720. 68

[Alfvén, 1939] Alfvén, Hannes. 1939. On the Motion of Cosmic Rays in Interstellar Space. Phys. Rev., 55(Mar), 425-429. 43

[Amaldi \& Kraft, 2005] Amaldi, Ugo, \& Kraft, Gerhard. 2005. Radiotherapy with beams of carbon ions. Reports on Progress in Physics, 68(8), 1861-1882. 25

[Andersen \& Ziegler, n.d.] Andersen, H H, \& Ziegler, J F. Hydrogen. Stopping powers and ranges in all elements. [10 $\mathrm{keV}$ to $20 \mathrm{MeV}$ (per amu); book]. 80

[Andersen \& Ziegler, 1977] Andersen, H. H. (Hans Henrik), \& Ziegler, J. F. (James F.), (joint author.). 1977. Hydrogen stopping powers and ranges in all elements. New York : Pergamon Press. Includes bibliographical references. 57

[Apiñaniz et al., 2021] Apiñaniz, J. I., Malko, S., Fedosejevs, R., Cayzac, W., Vaisseau, X., de Luis, D., Gatti, G., McGuffey, C., Bailly-Grandvaux, M., Bhutwala, K., Ospina-Bohorquez, V., Balboa, J., Santos, J. J., Batani, D., Beg, F., Roso, L., Pérez-Hernández, J. A., \& Volpe, L. 2021. A quasi-monoenergetic short time duration compact proton source for probing high energy density states of matter. Scientific Reports, 11(1), 6881. 3

[Badhwar et al., 1967] Badhwar, G.D., Deney, C.L., Dennis, B.R., \& Kaplon, M.F. 1967. The non-linear response of the plastic scintillator NE102. Nuclear Instruments and Methods, 57, 116-120. 81, 88 
[Barberio \& Antici, 2019] Barberio, M., \& Antici, P. 2019. Laser-PIXE using laseraccelerated proton beams. Nature Communications, 9, 6855. 2

[Barkas et al., 1956] Barkas, Walter H., Birnbaum, Wallace, \& Smith, Frances M. 1956. Mass-Ratio Method Applied to the Measurement of $L$-Meson Masses and the Energy Balance in Pion Decay. Phys. Rev., 101(Jan), 778-795. 57

[Barkas et al., 1963] Barkas, Walter H., Dyer, John N., \& Heckman, Harry H. 1963. Resolution of the $\Sigma^{-}$-Mass Anomaly. Phys. Rev. Lett., 11(Jul), 26-28. 57

[Basov \& Prokhorov, 1956] Basov, N. G., \& Prokhorov, A.M. 1956. Theory of the Molecular Generator and Molecular Power Amplifier. J. Exptl. Theoret. Phys., 30(March), 560-563. 7

[Battistoni et al., 2007] Battistoni, G., Cerutti, F., Fass, A., Ferrari, A., Muraro, S., Ranft, J., Roesler, S., \& Sala, P. R. 2007. The FLUKA code: description and benchmarking. AIP Conference Proceedings, 896(1), 31-49. 80

[Battistoni et al., 2016] Battistoni, Giuseppe, Bauer, Julia, Boehlen, Till T., Cerutti, Francesco, Chin, Mary P. W., Dos Santos Augusto, Ricardo, Ferrari, Alfredo, Ortega, Pablo G., Kozowska, Wioletta, Magro, Giuseppe, Mairani, Andrea, Parodi, Katia, Sala, Paola R., Schoofs, Philippe, Tessonnier, Thomas, \& Vlachoudis, Vasilis. 2016. The FLUKA Code: An Accurate Simulation Tool for Particle Therapy. Frontiers in Oncology, 6, 116. 80

[Beg et al., 1997] Beg, F. N., Bell, A. R., Dangor, A. E., Danson, C. N., Fews, A. P., Glinsky, M. E., Hammel, B. A., Lee, P., Norreys, P. A., \& Tatarakis, M. 1997. A study of picosecond lasersolid interactions up to $1019 \mathrm{Wcm} 2$. Physics of Plasmas, 4(2), 447-457. 23, 36

[Bell, 1948] Bell, P. R. 1948. The Use of Anthracene as a Scintillation Counter. Physical Review, 73, 1405-1406. 68

[Berger \& Seltzer, 1964] Berger, M. J., \& Seltzer, S. M. 1964. Tables of energy losses and ranges of electrons and positrons. NASA technical report SP-3012. 42

[Berger et al., 1993a] Berger, M. J., Inokuti, M., Andersen, H. H., Bichsel, H., Powers, D., Seltzer, S . M., Thwaites, D ., \& Watt, D. E. 1993a. Report 49. Journal of the International Commission on Radiation Units and Measurements, os25(2), NP-NP. 56

[Berger et al., 1993b] Berger, M. J., Inokuti, M., Andersen, H. H., Bichsel, H., Powers, D., Seltzer, S . M., Thwaites, D ., \& Watt, D. E. 1993b. Report 49. Journal of the International Commission on Radiation Units and Measurements, os25(2), NP-NP. 80

[Bethe, 1930] Bethe, H. 1930. Zur Theorie des Durchgangs schneller Korpuskularstrahlen durch Materie. Annalen der Physik, 397(3), 325-400. 55 
[Bethe, 1932] Bethe, H. 1932. Bremsformel fr Elektronen relativistischer Geschwindigkeit. Zeitschrift fr Physik, 76(Jan), 293-299. 40

[Bethe, 1933] Bethe, H. 1933. Quantenmechanik der Ein- und Zwei-Elektronen Probleme. Handbuch derPhysik, 24/1, 273P. 55, 56

[Bethe, 1953] Bethe, H. A. 1953. Molière's Theory of Multiple Scattering. Phys. Rev., 89(Mar), 1256-1266. 57

[Bethe \& Ashkin, 1953] Bethe, Hans A, \& Ashkin, Julius. 1953. Experimental nuclear physics. Wiley, New York. 56

[Bichsel, 1991] Bichsel, H. 1991 (Apr). Stopping power of fast charged particles in heavy elements. Tech. rept. 57

[Bichsel, 1983] Bichsel, Hans. 1983. Stopping power of $M$-shell electrons for heavy charged particles. Phys. Rev. A, 28(Aug), 1147-1150. 57

[Bichsel, 1992] Bichsel, Hans. 1992. Stopping power and ranges of fast ions in heavy elements. Phys. Rev. A, 46(Nov), 5761-5773. 57

[Birks, 1951] Birks, J. B. 1951. Scintillations from Organic Crystals: Specific Fluorescence and Relative Response to Different Radiations. Proceedings of the Physical Society. Section A, 64(10), 874-877. 71

[Birks, 1964] Birks, J.B. 1964. CHAPTER 3 - The scintillation process in organic materials - I. Pages 39-67 of: Birks, J.B. (ed), The Theory and Practice of Scintillation Counting. International Series of Monographs in Electronics and Instrumentation. Pergamon. 69

[Bloch, 1933] Bloch, F. 1933. Zur Bremsung rasch bewegter Teilchen beim Durchgang durch Materie. Annalen der Physik, 408(3), 285-320. 57

[Bohr, 1913] Bohr, N. 1913. On the theory of the decrease of velocity of moving electrified particles on passing through matter. The London, Edinburgh, and Dublin Philosophical Magazine and Journal of Science, 25(145), 10-31. 55

[Bohr, 1948] Bohr, Niels. 1948. The penetration of atomic particles through matter. Matt.-Fys. Medd., 18. 57

[Borghesi et al., 2003] Borghesi, M., Schiavi, A., Campbell, D. H., Haines, M. G., Willi, O., Mackinnon, A. J., Patel, P., Galimberti, M., \& Gizzi, L. A. 2003. Proton imaging detection of transient electromagnetic fields in laser-plasma interactions (invited). Review of Scientific Instruments, 74(3), 1688-1693. 24

[Brannen \& Olde, 1962] Brannen, Eric, \& Olde, Garth L. 1962. The Response of Organic Scintillators to Electron Energy Deposited in Them. Radiation Research, 16(1), 1-6. 71 
[Broser \& Kallmann, 1947] Broser, I., \& Kallmann, H. 1947. Über den Elementarprozeß der Lichtanregung in Leuchtstoffen durch $a$ Teilchen, schnelle Elektronen und $\gamma$ Quanten II. Z. Naturforschg., 2a, 642-650. 68

[Brown et al., 2008] Brown, C G, Throop, A, Eder, D, \& Kimbrough, J. 2008. Electromagnetic pulses at short-pulse laser facilities. Journal of Physics: Conference Series, 112(3), 032025. 65

[Brunel, 1987] Brunel, F. 1987. Not-so-resonant, resonant absorption. Phys. Rev. Lett., 59(Jul), 52-55. 34

[Buffechoux et al., 2010] Buffechoux, S., Psikal, J., Nakatsutsumi, M., Romagnani, L., Andreev, A., Zeil, K., Amin, M., Antici, P., Burris-Mog, T., Compant-LaFontaine, A., d'Humières, E., Fourmaux, S., Gaillard, S., Gobet, F., Hannachi, F., Kraft, S., Mancic, A., Plaisir, C., Sarri, G., Tarisien, M., Toncian, T., Schramm, U., Tampo, M., Audebert, P., Willi, O., Cowan, T. E., Pépin, H., Tikhonchuk, V., Borghesi, M., \& Fuchs, J. 2010. Hot Electrons Transverse Refluxing in Ultraintense Laser-Solid Interactions. Phys. Rev. Lett., 105(Jul), 015005. 53, 54

[Bulanov et al., 2002] Bulanov, S.V, Esirkepov, T.Zh, Khoroshkov, V.S, Kuznetsov, A.V, \& Pegoraro, F. 2002. Oncological hadrontherapy with laser ion accelerators. Physics Letters A, 299(2), 240 - 247. 24

[Bulanov \& Khoroshkov, n.d.] Bulanov, S V, Wilkens J J Molls M Esirkepov T Zh Korn G Kraft G Kraft S D, \& Khoroshkov, V S. Laser ion acceleration for hadron therapy. 2

[Butson et al., 2003] Butson, Martin J, Yu, Peter K.N, Cheung, Tsang, \& Metcalfe, Peter. 2003. Radiochromic film for medical radiation dosimetry. Materials Science and Engineering: R: Reports, 41(3), $61-120.65$

[Bychenkov et al., 2001] Bychenkov, V. Yu., Rozmus, W., Maksimchuk, A., Umstadter, D., \& Capjack, C. E. 2001. Fast ignitor concept with light ions. Plasma Physics Reports, 27, 10171020. 24

[Ceccotti et al., 2007] Ceccotti, T., Lévy, A., Popescu, H., Réau, F., D’Oliveira, P., Monot, P., Geindre, J. P., Lefebvre, E., \& Martin, Ph. 2007. Proton Acceleration with High-Intensity Ultrahigh-Contrast Laser Pulses. Phys. Rev. Lett., 99(Oct), 185002. 53

[Chopineau et al., 2019] Chopineau, L., Leblanc, A., Blaclard, G., Denoeud, A., Thévenet, M., Vay, J-L., Bonnaud, G., Martin, Ph., Vincenti, H., \& Quéré, F. 2019. Identification of Coupling Mechanisms between Ultraintense Laser Light and Dense Plasmas. Phys. Rev. X, 9(Mar), 011050. 35

[Clark et al., 2000] Clark, E. L., Krushelnick, K., Davies, J. R., Zepf, M., Tatarakis, M., Beg, F. N., Machacek, A., Norreys, P. A., Santala, M. I. K., Watts, I., \& Dangor, A. E. 2000. Measurements of Energetic Proton Transport through Magnetized 
Plasma from Intense Laser Interactions with Solids. Phys. Rev. Lett., 84(Jan), 670-673. 1, 23

[Climent-Font et al., 2004] Climent-Font, A., Pászti, F., García, G., FernándezJiḿnez, M.T., \& Agulló, F. 2004. First measurements with the Madrid 5 MV tandem accelerator. Nuclear Instruments and Methods in Physics Research Section B: Beam Interactions with Materials and Atoms, 219-220, 400 - 404. Proceedings of the Sixteenth International Conference on Ion Beam Analysis. 81

[CMAM, 2021] CMAM. 2021. Website of the CMAM center: https://www. cmam. uam. es/. 82

[Couperus, 2018] Couperus, Jurjen Pieter. 2018. Optimal beam loading in a nanocoulomb-class laser wakefield accelerator. Ph.D. thesis. 140

[Cowan et al., 2004] Cowan, T. E., Fuchs, J., Ruhl, H., Kemp, A., Audebert, P., Roth, M., Stephens, R., Barton, I., Blazevic, A., Brambrink, E., Cobble, J., Fernández, J., Gauthier, J.-C., Geissel, M., Hegelich, M., Kaae, J., Karsch, S., Le Sage, G. P., Letzring, S., Manclossi, M., Meyroneinc, S., Newkirk, A., Pépin, H., \& RenardLeGalloudec, N. 2004. Ultralow Emittance, Multi-MeV Proton Beams from a Laser Virtual-Cathode Plasma Accelerator. Phys. Rev. Lett., 92(May), 204801. 50, 51, 124

[Craun \& Smith, 1970] Craun, R.L., \& Smith, D.L. 1970. Analysis of response data for several organic scintillators. Nuclear Instruments and Methods, 80(2), 239-244. 81,88

[Crookes, 1902] Crookes, W. 1902. The Emanations of Radium. Proceedings of the Royal Society of London, 71, 405-408. 67

[Curcio \& Volpe, 2019] Curcio, A, \& Volpe, L. 2019. A quasi-static model for hot-electron interaction with self-generated magnetic fields. Plasma Physics and Controlled Fusion, 61(5), 055013. 44

[Davies, 2008] Davies, J R. 2008. Laser absorption by overdense plasmas in the relativistic regime. Plasma Physics and Controlled Fusion, 51(1), 014006. 36

[Davies et al., 1997] Davies, J. R., Bell, A. R., Haines, M. G., \& Guérin, S. M. 1997. Short-pulse high-intensity laser-generated fast electron transport into thick solid targets. Phys. Rev. E, 56(Dec), 7193-7203. 44

[Debayle et al., 2010] Debayle, A., Honrubia, J. J., d'Humières, E., \& Tikhonchuk, V. T. 2010. Divergence of laser-driven relativistic electron beams. Phys. Rev. E, 82(Sep), 036405. 123

[Drake et al., 1974] Drake, J. F., Kaw, P. K., Lee, Y. C., Schmid, G., Liu, C. S., \& Rosenbluth, Marshall N. 1974. Parametric instabilities of electromagnetic waves in plasmas. The Physics of Fluids, 17(4), 778-785. 35 
[Ebenau et al., 2016] Ebenau, Melanie, Radeck, Dsire, Bambynek, Markus, Sommer, Holger, Flhs, Dirk, Spaan, Bernhard, \& Eichmann, Marion. 2016. Energy dependent response of plastic scintillation detectors to photon radiation of low to medium energy. Medical Physics, 43(8Part1), 4598-4606. 81

[Einstein, 1917] Einstein, A. 1917. Zur Quantentheorie der Strahlung. Physikalische Zeitschrift, 18, 121-128. 7

[Fassò et al., 1995] Fassò, A., Ferrari, A., Ranft, J., \& Sala, P. 1995. AN UPDATE ABOUT FLUKA. 80

[Feng et al., 2018] Feng, Yiwei, Tiedje, Henry F., Gagnon, Katherine, \& Fedosejevs, Robert. 2018. Spectral calibration of EBT3 and HD-V2 radiochromic film response at high dose using $20 \mathrm{MeV}$ proton beams. Review of Scientific Instruments, 89(4), 043511. 65

[Fermi, 1940] Fermi, Enrico. 1940. The Ionization Loss of Energy in Gases and in Condensed Materials. Phys. Rev., 57(Mar), 485-493. 41, 57

[Ferrari et al., 1992] Ferrari, A., Sala, P.R., Guaraldi, R., \& Padoani, F. 1992. An improved multiple scattering model for charged particle transport. Nuclear Instruments and Methods in Physics Research Section B: Beam Interactions with Materials and Atoms, 71(4), 412 - 426. 80

[Ferrari et al., 2005] Ferrari, A, Sala, P R, /CERN /INFN, Milan, Fasso, A, /SLAC, Ranft, J, \& U., /Siegen. 2005. FLUKA: A Multi-Particle Transport Code. 12. 80

[Fill, 2005] Fill, Ernst E. 2005. Ultrashort-pulse laser plasmas: Fraction of hot electrons escaping from the target and electron spectra in planar and spherical geometry. Physics of Plasmas, 12(5), 052704. 45

[Flacco et al., 2010] Flacco, A., Sylla, F., Veltcheva, M., Carrié, M., Nuter, R., Lefebvre, E., Batani, D., \& Malka, V. 2010. Dependence on pulse duration and foil thickness in high-contrast-laser proton acceleration. Phys. Rev. E, 81(Mar), 036405. 53

[Fleischer et al., 1965] Fleischer, R. L., Price, P. B., \& Walker, R. M. 1965. Ion Explosion Spike Mechanism for Formation of ChargedParticle Tracks in Solids. Journal of Applied Physics, 36(11), 3645-3652. 65

[Flippo et al., 2008] Flippo, K. A., dHumires, E., Gaillard, S. A., Rassuchine, J., Gautier, D. C., Schollmeier, M., Nrnberg, F., Kline, J. L., Adams, J., Albright, B., Bakeman, M., Harres, K., Johnson, R. P., Korgan, G., Letzring, S., Malekos, S., Renard-LeGalloudec, N., Sentoku, Y., Shimada, T., Roth, M., Cowan, T. E., Fernndez, J. C., \& Hegelich, B. M. 2008. Increased efficiency of short-pulse lasergenerated proton beams from novel flat-top cone targets. Physics of Plasmas, 15(5), 056709. 53 
[Forslund et al., 1975] Forslund, D. W., Kindel, J. M., Lee, Kenneth, Lindman, E. L., \& Morse, R. L. 1975. Theory and simulation of resonant absorption in a hot plasma. Phys. Rev. A, 11(Feb), 679-683. 32

[Forslund et al., 1977] Forslund, D. W., Kindel, J. M., \& Lee, K. 1977. Theory of Hot-Electron Spectra at High Laser Intensity. Phys. Rev. Lett., 39(Aug), 284-288. 33

[Fourkal et al., 2002] Fourkal, E., Shahine, B., Ding, M., Li, J. S., Tajima, T., \& Ma, C.-M. 2002. Particle in cell simulation of laser-accelerated proton beams for radiation therapy. Medical Physics, 29(12), 2788-2798. 24

[Freidberg et al., 1972] Freidberg, J. P., Mitchell, R. W., Morse, R. L., \& Rudsinski, L. I. 1972. Resonant Absorption of Laser Light by Plasma Targets. Phys. Rev. Lett., 28(Mar), 795-799. 32

[Fritzler et al., 2003] Fritzler, S., Malka, V., Grillon, G., Rousseau, J. P., Burgy, F., Lefebvre, E., dHumires, E., McKenna, P., \& Ledingham, K. W. D. 2003. Proton beams generated with high-intensity lasers: Applications to medical isotope production. Applied Physics Letters, 83(15), 3039-3041. 2, 24

[Fuchs et al., 2003] Fuchs, J., Cowan, T. E., Audebert, P., Ruhl, H., Gremillet, L., Kemp, A., Allen, M., Blazevic, A., Gauthier, J.-C., Geissel, M., Hegelich, M., Karsch, S., Parks, P., Roth, M., Sentoku, Y., Stephens, R., \& Campbell, E. M. 2003. Spatial Uniformity of Laser-Accelerated Ultrahigh-Current MeV Electron Propagation in Metals and Insulators. Phys. Rev. Lett., 91(Dec), 255002. 123

[Fuchs et al., 2006] Fuchs, J., Antici, P., dH́umiéres, E., Lefebvre, E., Borghesi, M., Brambrink, E., Cecchetti, C. A., Kaluza, M., Malka, V., Manclossi, M., Meyroneinc, S., Mora, P., Schreiber, J., Toncian, T., Ppin, H., \& Audebert, P. 2006. Laser-driven proton scaling laws and new paths towards energy increase. Nature Phys, 2(jan), 4854. 54

[Gafchromic, 2020] Gafchromic, Ashland. 2020. Available at http: //www. gafchromic. com/gafchromic-film/index. asp and http: //www. gafchromic. com/documents/gafchromic-hdv2.pdf for HDV2 Gafchromic films. 66, 102

[Gaillard et al., 2007] Gaillard, S., Fuchs, J., Renard-Le Galloudec, N., \& Cowan, T. E. 2007. Study of saturation of CR39 nuclear track detectors at high ion fluence and of associated artifact patterns. Review of Scientific Instruments, 78(1), 013304. 65

[Gaillard et al., 2011] Gaillard, S. A., Kluge, T., Flippo, K. A., Bussmann, M., Gall, B., Lockard, T., Geissel, M., Offermann, D. T., Schollmeier, M., Sentoku, Y., \& 
Cowan, T. E. 2011. Increased laser-accelerated proton energies via direct laserlight-pressure acceleration of electrons in microcone targets. Physics of Plasmas, 18(5), 056710. 2, 54

[Gibbon, 2005] Gibbon, Paul. 2005. Short Pulse Laser Interactions with Matter. imperial college press. $14,33,34$

[Gitomer et al., 1986] Gitomer, S. J., Jones, R. D., Begay, F., Ehler, A. W., Kephart, J. F., \& Kristal, R. 1986. Fast ions and hot electrons in the laserplasma interaction. The Physics of Fluids, 29(8), 2679-2688. 24, 65

[Gooding \& Pugh, 1960] Gooding, T.J., \& Pugh, H.G. 1960. The response of plastic scintillators to high-energy particles. Nuclear Instruments and Methods, 7(2), 189192. 88

[Gordon et al., 1955] Gordon, J. P., Zeiger, H. J., \& Townes, C. H. 1955. The Maser: New Type of Microwave Amplifier, Frequency Standard, and Spectrometer. Phys. Rev., 99(Aug), 1264-1274. 7

[Goudsmit \& Saunderson, 1940] Goudsmit, S., \& Saunderson, J. L. 1940. Multiple Scattering of Electrons. Phys. Rev., 57(Jan), 24-29. 57

[Green et al., 2008] Green, J. S., Ovchinnikov, V. M., Evans, R. G., Akli, K. U., Azechi, H., Beg, F. N., Bellei, C., Freeman, R. R., Habara, H., Heathcote, R., Key, M. H., King, J. A., Lancaster, K. L., Lopes, N. C., Ma, T., MacKinnon, A. J., Markey, K., McPhee, A., Najmudin, Z., Nilson, P., Onofrei, R., Stephens, R., Takeda, K., Tanaka, K. A., Theobald, W., Tanimoto, T., Waugh, J., Van Woerkom, L., Woolsey, N. C., Zepf, M., Davies, J. R., \& Norreys, P. A. 2008. Effect of Laser Intensity on Fast-Electron-Beam Divergence in Solid-Density Plasmas. Phys. Rev. Lett., 100(Jan), 015003. 37, 38

[Green et al., 2011] Green, J. S., Borghesi, M., Brenner, C. M., Carroll, D. C., Dover, N. P., Foster, P. S., Gallegos, P., Green, S., Kirby, D., Kirkby, K. J., McKenna, P., Merchant, M. J., Najmudin, Z., Palmer, C. A. J., Parker, D., Prasad, R., Quinn, K. E., Rajeev, P. P., Read, M. P., Romagnani, L., Schreiber, J., Streeter, M. J. V., Tresca, O., Wahlstrm, C.-G., Zepf, M., \& Neely, David. 2011. Scintillator-based ion beam profiler for diagnosing laser-accelerated ion beams. Pages 147-154 of: Leemans, Wim P., Esarey, Eric, Hooker, Simon M., \& Ledingham, Kenneth W. D. (eds), Laser Acceleration of Electrons, Protons, and Ions; and Medical Applications of Laser-Generated Secondary Sources of Radiation and Particles, vol. 8079SPIE, for International Society for Optics and Photonics. 75

[Green et al., 2014] Green, J. S., Robinson, A. P. L., Booth, N., Carroll, D. C., Dance, R. J., Gray, R. J., MacLellan, D. A., McKenna, P., Murphy, C. D., Rusby, D., \& Wilson, L. 2014. High efficiency proton beam generation through target thickness control in femtosecond laser-plasma interactions. Applied Physics Letters, 104(21), 214101. 54, 65 
[Gremillet, 2001] Gremillet, Laurent. 2001. Etude exprimentale et numrique du transport des lectrons rapides dans l'interaction laser - solide trs haut flux. Theses, Ecole Polytechnique. 37

[Guemnie-Tafo, 2007] Guemnie-Tafo, Alain. 2007. Faisceau de protons generes par linteraction dun laser ultra court avec une cible solide. Theses, Ecole Polytechnique. 13

[Hammer \& Rostoker, 1970] Hammer, D. A., \& Rostoker, N. 1970. Propagation of High Current Relativistic Electron Beams. The Physics of Fluids, 13(7), 18311850. 43

[Hargrove et al., 1964] Hargrove, L. E., Fork, R. L., \& Pollack, M. A. 1964. LOCKING OF HeNe LASER MODES INDUCED BY SYNCHRONOUS INTRACAVITY MODUlation. Applied Physics Letters, 5(1), 4-5. 11

[Hatchett et al., 2000] Hatchett, Stephen P., Brown, Curtis G., Cowan, Thomas E., Henry, Eugene A., Johnson, Joy S., Key, Michael H., Koch, Jeffrey A., Langdon, A. Bruce, Lasinski, Barbara F., Lee, Richard W., Mackinnon, Andrew J., Pennington, Deanna M., Perry, Michael D., Phillips, Thomas W., Roth, Markus, Sangster, T. Craig, Singh, Mike S., Snavely, Richard A., Stoyer, Mark A., Wilks, Scott C., \& Yasuike, Kazuhito. 2000. Electron, photon, and ion beams from the relativistic interaction of Petawatt laser pulses with solid targets. Physics of Plasmas, 7(5), 2076-2082. 49

[Hegelich et al., 2002] Hegelich, M., Karsch, S., Pretzler, G., Habs, D., Witte, K., Guenther, W., Allen, M., Blazevic, A., Fuchs, J., Gauthier, J. C., Geissel, M., Audebert, P., Cowan, T., \& Roth, M. 2002. MeV Ion Jets from Short-Pulse-Laser Interaction with Thin Foils. Phys. Rev. Lett., 89(Aug), 085002. 49, 50

[Heitler \& Sauter, 1933] Heitler, W., \& Sauter, F. 1933. Stopping of Fast Particles with Emission of Radiation and the Birth of Positive Electrons. Nature, 132(892). 42

[Henig et al., 2009] Henig, A., Kiefer, D., Geissler, M., Rykovanov, S. G., Ramis, R., Hörlein, R., Osterhoff, J., Major, Zs., Veisz, L., Karsch, S., Krausz, F., Habs, D., \& Schreiber, J. 2009. Laser-Driven Shock Acceleration of Ion Beams from Spherical Mass-Limited Targets. Phys. Rev. Lett., 102(Mar), 095002. 53

[Higginson et al., 2018] Higginson, A., Gray, R. J., King, M., Dance, R. J., Williamson, S. D. R., Butler, N. M. H., Wilson, R., Capdessus, R., Armstrong, C., Green, J. S., Hawkes, S. J., Martin, P., Wei, W. Q., Mirfayzi, S. R., Yuan, X. H., Kar, S., Borghesi, M., Clarke, R. J., Neely, D., \& McKenna, P. 2018. Near-100 $\mathrm{MeV}$ protons via a laser-driven transparency-enhanced hybrid acceleration scheme. Nature Communications, 9, 724. 2 
[Highland, 1975] Highland, Virgil L. 1975. Some practical remarks on multiple scattering. Nuclear Instruments and Methods, 129(2), 497-499. 57

[Huault et al., 2019] Huault, M., De Luis, D., Apiñaniz, J. I., De Marco, M., Salgado, C., Gordillo, N., Gutiérrez Neira, C., Pérez-Hernández, J. A., Fedosejevs, R., Gatti, G., \& et al. 2019. A 2D scintillator-based proton detector for high repetition rate experiments. High Power Laser Science and Engineering, 7, e60. 4

[ISO/ASTM51275, 2013] ISO/ASTM51275. 2013. Practice for use of a radiochromic film dosimetry system. 65

[Kallmann, 1950] Kallmann, H. 1950. Scintillation Counting with Solutions. Physical Review, 78(Jun), 621-622. 68

[Kaluza et al., 2004] Kaluza, M., Schreiber, J., Santala, M. I. K., Tsakiris, G. D., Eidmann, K., Meyer-ter Vehn, J., \& Witte, K. J. 2004. Influence of the Laser Prepulse on Proton Acceleration in Thin-Foil Experiments. Phys. Rev. Lett., 93(Jul), 045003. 51, 52, 53, 127

[Kastler, 1950] Kastler, Alfred. 1950. Quelques suggestions concernant la production optique et la détection optique d'une inégalité de population des niveaux de quantifigation spatiale des atomes. Application à l'expérience de Stern et Gerlach et à la résonance magnétique. J. Phys. Radium, 11(6), 255-265. 7

[Kaufman et al., 2015] Kaufman, J., Margarone, Daniele, Candiano, Giacomo, Kim, I Jong, Jeong, Tae Moon, Pikal, Jan, Romano, F., Cirrone, P., Scuderi, V., \& Korn, Georg. 2015. Radiochromic film diagnostics for laser-driven ion beams. Pages $174-$ 181 of: Korn, Georg, \& Silva, Luis O. (eds), Research Using Extreme Light: Entering New Frontiers with Petawatt-Class Lasers II, vol. 9515SPIE, for International Society for Optics and Photonics. 102

[Keldysh, 1965] Keldysh, L. V. 1965. Ionization in the Field of a Strong Electromagnetic Wave. JETP, 20(5), 1307. 14

[Kellogg et al., 1976] Kellogg, E., Henry, P., Murray, S., Van Speybroeck, L., \& Bjorkholm, P. 1976. Highresolution imaging xray detector. Review of Scientific Instruments, 47(3), 282-290. 59

[Khandelwal, 1968] Khandelwal, Govind S. 1968. Shell corrections for K- and Lelectrons. Nucl. Phys., A116, 97-111. 57

[Kim et al., 2013] Kim, I Jong, Pae, Ki Hong, Kim, Chul Min, Kim, Hyung Taek, Sung, Jae Hee, Lee, Seong Ku, Yu, Tae Jun, Choi, Il Woo, Lee, Chang-Lyoul, Nam, Kee Hwan, Nickles, Peter V., Jeong, Tae Moon, \& Lee, Jongmin. 2013. Transition of Proton Energy Scaling Using an Ultrathin Target Irradiated by Linearly Polarized Femtosecond Laser Pulses. Phys. Rev. Lett., 111(Oct), 165003. 54 
[Knoll, 2010] Knoll, G. F. 2010. Radiation Detection and Measurement. 3rd edn. John Wilsey \& Sons. Page 219, Chapter 8, Scintillation Detector Principles. 67, 70,71

[Koenig et al., 2005] Koenig, M, Benuzzi-Mounaix, A, Ravasio, A, Vinci, T, Ozaki, N, Lepape, S, Batani, D, Huser, G, Hall, T, Hicks, D, MacKinnon, A, Patel, P, Park, H S, Boehly, T, Borghesi, M, Kar, S, \& Romagnani, L. 2005. Progress in the study of warm dense matter. Plasma Physics and Controlled Fusion, 47(12B), B441-B449. 24

[Kruer, 1988] Kruer, W. L. 1988. The Physics of Laser Plasma Interactions. Addisonwesley,new-york edn. Avalon Publishing. 32

[Kruer \& Estabrook, 1985] Kruer, W. L., \& Estabrook, Kent. 1985. JB heating by very intense laser light. The Physics of Fluids, 28(1), 430-432. 34

[Krushelnick et al., 1999] Krushelnick, K., Clark, E. L., Najmudin, Z., Salvati, M., Santala, M. I. K., Tatarakis, M., Dangor, A. E., Malka, V., Neely, D., Allott, R., \& Danson, C. 1999. Multi-MeV Ion Production from High-Intensity Laser Interactions with Underdense Plasmas. Phys. Rev. Lett., 83(Jul), 737-740. 23

[Ladislas Wiza, 1979] Ladislas Wiza, Joseph. 1979. Microchannel plate detectors. Nuclear Instruments and Methods, 162(1), 587 - 601. 60, 61

[Lawson, 1959] Lawson, J D. 1959. On the classification of electron streams. Journal of Nuclear Energy. Part C, Plasma Physics, Accelerators, Thermonuclear Research, 1(1), 31-35. 43

[Lawson, 1979] Lawson, J D. 1979. Lasers and Accelerators. IEEE Transactions on Nuclear Science, 26(3), 4217-4219. 29

[Lee \& Sudan, 1971] Lee, Roswell, \& Sudan, R. N. 1971. Return Current Induced by a Relativistic Beam Propagating in a Magnetized Plasma. The Physics of Fluids, 14(6), 1213-1225. 43

[Lindhard \& Winther, 1964] Lindhard, J., \& Winther, A. 1964. Stopping Power of Electron Gas and Equipartition Rule. Kgl. Danske Videnskab. Selskab Mat.-fys. Medd., 34(4). 57

[Lontano \& Passoni, 2006] Lontano, Maurizio, \& Passoni, Matteo. 2006. Electrostatic field distribution at the sharp interface between high density matter and vacuum. Physics of Plasmas, 13(4), 042102. 47

[Lundh et al., 2007] Lundh, O., Lindau, F., Persson, A., Wahlström, C.-G., McKenna, P., \& Batani, D. 2007. Influence of shock waves on laser-driven proton acceleration. Phys. Rev. E, 76(Aug), 026404. 124 
[Lynch \& Dahl, 1991] Lynch, Gerald R., \& Dahl, Orin I. 1991. Approximations to multiple Coulomb scattering. Nuclear Instruments and Methods in Physics Research Section B: Beam Interactions with Materials and Atoms, 58(1), 6-10. 57, 58,111

[Macchi et al., 2013] Macchi, Andrea, Borghesi, Marco, \& Passoni, Matteo. 2013. Ion acceleration by superintense laser-plasma interaction. Rev. Mod. Phys., 85(May), 751-793. 25

[Mackinnon et al., 2001] Mackinnon, A. J., Borghesi, M., Hatchett, S., Key, M. H., Patel, P. K., Campbell, H., Schiavi, A., Snavely, R., Wilks, S. C., \& Willi, O. 2001. Effect of Plasma Scale Length on Multi-MeV Proton Production by Intense Laser Pulses. Phys. Rev. Lett., 86(Feb), 1769-1772. 49

[Mackinnon et al., 2002] Mackinnon, A. J., Sentoku, Y., Patel, P. K., Price, D. W., Hatchett, S., Key, M. H., Andersen, C., Snavely, R., \& Freeman, R. R. 2002. Enhancement of Proton Acceleration by Hot-Electron Recirculation in Thin Foils Irradiated by Ultraintense Laser Pulses. Phys. Rev. Lett., 88(May), 215006. 45, 51

[Maiman, 1960] Maiman, T. H. 1960. Stimulated Optical Radiation in Ruby. Nature, 187, 493494. 7

[Maksimchuk et al., 2000] Maksimchuk, A., Gu, S., Flippo, K., Umstadter, D., \& Bychenkov, V. Yu. 2000. Forward Ion Acceleration in Thin Films Driven by a High-Intensity Laser. Phys. Rev. Lett., 84(May), 4108-4111. 1, 23

[Malka et al., 2004] Malka, Victor, Fritzler, Sven, Lefebvre, Erik, d'Humires, Emmanuel, Ferrand, Rgis, Grillon, Georges, Albaret, Claude, Meyroneinc, Samuel, Chambaret, Jean-Paul, Antonetti, Andre, \& Hulin, Danile. 2004. Practicability of protontherapy using compact laser systems. Medical Physics, 31(6), 1587-1592. 24

[Marco et al., 2016] Marco, M. De, Krása, J., Cikhardt, J., Pfeifer, M., Krouský, E., Margarone, D., Ahmed, H., Borghesi, M., Kar, S., Giuffrida, L., Vrana, R., Velyhan, A., Limpouch, J., Korn, G., Weber, S., Velardi, L., Side, D. Delle, Nassisi, V., \& Ullschmied, J. 2016. Measurement of electromagnetic pulses generated during interactions of high power lasers with solid targets. Journal of Instrumentation, 11(06), C06004-C06004. 58

[Margarone et al., 2011] Margarone, D., Krsa, J., Giuffrida, L., Picciotto, A., Torrisi, L., Nowak, T., Musumeci, P., Velyhan, A., Prokpek, J., Lska, L., Mocek, T., Ullschmied, J., \& Rus, B. 2011. Full characterization of laser-accelerated ion beams using Faraday cup, silicon carbide, and single-crystal diamond detectors. Journal of Applied Physics, 109(10), 103302. 59

[McClung \& Hellwarth, 1962] McClung, F. J., \& Hellwarth, R. W. 1962. Giant Optical Pulsations from Ruby. Journal of Applied Physics, 33(3), 828-829. 11 
[Méndez et al., 2019] Méndez, C., Varela, O., García, E., Hernández, I., Ajates, J., Pisonero, J. D., Sagredo, J. L., Olivar, M., \& Roso, L. 2019. VEGA laser facility beamlines management for pump-probe experiments. Pages $431-436$ of: Costa, Manuel Filipe P. C. M. Martins (ed), Fourth International Conference on Applications of Optics and Photonics, vol. 11207SPIE, for International Society for Optics and Photonics. 116

[Mendonça \& Doveil, 1982] Mendonça, J. T., \& Doveil, F. 1982. Stochasticity in plasmas with electromagnetic waves. Journal of Plasma Physics, 28(3), 485493. 35

[Metzkes et al., 2012] Metzkes, J., Karsch, L., Kraft, S. D., Pawelke, J., Richter, C., Schrer, M., Sobiella, M., Stiller, N., Zeil, K., \& Schramm, U. 2012. A scintillatorbased online detector for the angularly resolved measurement of laser-accelerated proton spectra. Review of Scientific Instruments, 83(12), 123301. 74, 75, 97

[Metzkes et al., 2016] Metzkes, J., Zeil, K., Kraft, S. D., Karsch, L., Sobiella, M., Rehwald, M., Obst, L., Schlenvoigt, H.-P., \& Schramm, U. 2016. An online, energyresolving beam profile detector for laser-driven proton beams. Review of Scientific Instruments, 87(8), 083310. 74, 75, 97

[Metzkes, 2015] Metzkes, Josefine. 2015. Studying the interaction of ultrashort, intense laser pulses with solid targets. Ph.D. thesis. 98

[Meyer \& Murray, 1962] Meyer, Axel, \& Murray, R. B. 1962. Effect of Energetic Secondary Electrons on the Scintillation Process in Alkali Halide Crystals. Phys. Rev., 128(Oct), 98-105. 81

[Mirani et al., 2021] Mirani, F., Maffini, A., Casamichiela, F., Pazzaglia, A., Formenti, A., Dellasega, D., Russo, V., Vavassori, D., Bortot, D., Huault, M., Zeraouli, G., Ospina, V., Malko, S., Apiñaniz, J. I., Pérez-Hernández, J. A., De Luis, D., Gatti, G., Volpe, L., Pola, A., \& Passoni, M. 2021. Integrated quantitative PIXE analysis and EDX spectroscopy using a laser-driven particle source. Science Advances, 7(3). 2

[Molière, 1947] Molière, Gert. 1947. Theorie der Streuung schneller geladener Teilchen I. Einzelstreuung am abgeschirmten Coulomb-Feld. Zeitschrift fr Naturforschung A, 2(3), 133 - 145. 39, 80

[Molière, 1948] Molière, Gert. 1948. Theorie der Streuung schneller geladener Teilchen II Mehrfach-und Vielfachstreuung. Zeitschrift fr Naturforschung A, 3(2), 78 - 97. $39,57,80$

[Molière, 1955] Molière, Gert. 1955. Theorie der streuung schneller geladener teilchen. III. die viel- fachstreuung von bahn-spuren unter bercksichtigung der statistischen kopplung. Zeitschrift fr Naturforschung A, 10, 177. 80 
[Møller, 1932] Møller, Chr. 1932. Zur Theorie des Durchgangs schneller Elektronen durch Materie. Annalen der Physik, 406(5), 531-585. 40

[Mora, 2003] Mora, P. 2003. Plasma Expansion into a Vacuum. Phys. Rev. Lett., 90(May), 185002. 45, 47, 48

[More, 1985] More, R.M. 1985. Pressure Ionization, Resonances, and the Continuity of Bound and Free States. Pages 305 - 356 of: Bates, D.R., \& Bederson, Benjamin (eds), Advances in Atomic and Molecular Physics. Advances in Atomic and Molecular Physics, vol. 21. Academic Press. 41

[Mulser \& Bauer, 1988] Mulser, P., \& Bauer, D. 1988. High power laser-matter interaction. Springer Tracts in Modern Physics. 30

[Myatt et al., 2007] Myatt, J., Theobald, W., Delettrez, J. A., Stoeckl, C., Storm, M., Sangster, T. C., Maximov, A. V., \& Short, R. W. 2007. High-intensity laser interactions with mass-limited solid targets and implications for fast-ignition experiments on OMEGA EP. Physics of Plasmas, 14(5), 056301. 45

[Nardi \& Zinamon, 1978] Nardi, E., \& Zinamon, Z. 1978. Energy deposition by relativistic electrons in high-temperature targets. Phys. Rev. A, 18(Sep), 12461249. 42

[Neumayer et al., 2010] Neumayer, P., Aurand, B., Basko, M., Ecker, B., Gibbon, P., Hochhaus, D. C., Karmakar, A., Kazakov, E., Khl, T., Labaune, C., Rosmej, O., Tauschwitz, An., Zielbauer, B., \& Zimmer, D. 2010. The role of hot electron refluxing in laser-generated K-alpha sources. Physics of Plasmas, 17(10), 103103. 35

[Newton et al., 1975] Newton, Marshall D., Lucas, Larry L., \& Root, John W. 1975. Proton stopping powers: Binary encounter calculations based on accurate speed distributions for target electrons. Chemical Physics Letters, 34(3), 552 - 556. 57

[Nicolaï et al., 2011] Nicolaï, Ph., Feugeas, J.-L., Regan, C., Olazabal-Loumé, M., Breil, J., Dubroca, B., Morreeuw, J.-P., \& Tikhonchuk, V. 2011. Effect of the plasma-generated magnetic field on relativistic electron transport. Phys. Rev. E, 84(Jul), 016402. 44

[Nigam et al., 1959] Nigam, B. P., Sundaresan, M. K., \& Wu, Ta-You. 1959. Theory of Multiple Scattering: Second Born Approximation and Corrections to Molière's Work. Phys. Rev., 115(Aug), 491-502. 39

[Obst et al., 2017] Obst, L., Göde, S., Rehwald, M., Brack, F.E., Branco, J., Bock, S., Bussmann, M., Cowan, T.E., Curry, C.B., Fiuza, F., Gauthier, M., Gebhardt, R., Helbig, U., Huebl, A., Hübner, U., Irman, A., Kazak, L., Kim, J.B., Kluge, T., Kraft, S., Loeser, M., Metzkes, J., Mishra, R., Rödel, C., Schlenvoigt, H.P., Siebold, M., Tiggesbäumker, J., Wolter, S., Ziegler, T., Schramm, U., Glenzer, 
S.H., \& Zeil, K. 2017. Efficient laser-driven proton acceleration from cylindrical and planar cryogenic hydrogen jets. Scientific Report, 7(1), 10248. 97, 99

[Obst et al., 2018] Obst, L., Metzkes-Ng, J., Bock, S., Cochran, G. E., Cowan, T. E., Oksenhendler, T., Poole, P. L., Prencipe, I., Rehwald, M., Rödel, C., Schlenvoigt, H-P., Schramm, U., Schumacher, D. W., Ziegler, T., \& Zeil, K. 2018. On-shot characterization of single plasma mirror temporal contrast improvement. Plasma Physics and Controlled Fusion, 60(5), 054007. 97, 139

[Ogura et al., 2012] Ogura, Koichi, Nishiuchi, Mamiko, Pirozhkov, Alexander S., Tanimoto, Tsuyoshi, Sagisaka, Akito, Esirkepov, Timur Zh., Kando, Masaki, Shizuma, Toshiyuki, Hayakawa, Takehito, Kiriyama, Hiromitsu, Shimomura, Takuya, Kondo, Shyuji, Kanazawa, Shuhei, Nakai, Yoshiki, Sasao, Hajime, Sasao, Fumitaka, Fukuda, Yuji, Sakaki, Hironao, Kanasaki, Masato, Yogo, Akifumi, Bulanov, Sergei V., Bolton, Paul R., \& Kondo, Kiminori. 2012. Proton acceleration to 40\&\#xA0;MeV using a high intensity, high contrast optical parametric chirpedpulse amplification/Ti:sapphire hybrid laser system. Opt. Lett., 37(14), 2868-2870. 54

[Pascual, 2004] Pascual, C. 2004. Experimental determination of stopping forces for ions in matter. Theses, Universidad Autónoma de Madrid. 81

[Perego et al., 2012] Perego, C., Batani, D., Zani, A., \& Passoni, M. 2012. Target normal sheath acceleration analytical modeling, comparative study and developments. Review of Scientific Instruments, 83(2), 02B502. 45

[Perego, 2013] Perego, Claudio. 2013. Target Normal Sheath Acceleration for LaserDriven Ion Generation: Advances in Theoretical Modeling. Theses, University of Milano-Bicocca. 47

[Perez, 2010] Perez, F. 2010. Étude du transport des électrons suprathermiques en milieu solide ou comprimé dans le cadre de lállumeur rapide. Theses, Ecole Polytechnique. 39,41

[Pines \& Bohm, 1952] Pines, David, \& Bohm, David. 1952. A Collective Description of Electron Interactions: II. Collective vs Individual Particle Aspects of the Interactions. Phys. Rev., 85(Jan), 338-353. 42

[Poyé et al., 2015] Poyé, A., Hulin, S., Bailly-Grandvaux, M., Dubois, J.-L., Ribolzi, J., Raffestin, D., Bardon, M., Lubrano-Lavaderci, F., D'Humières, E., Santos, J. J., Nicolaï, Ph., \& Tikhonchuk, V. 2015. Physics of giant electromagnetic pulse generation in short-pulse laser experiments. Phys. Rev. E, 91(Apr), 043106. 65

[Prasad et al., 2010] Prasad, R., Doria, D., Ter-Avetisyan, S., Foster, P.S., Quinn, K.E., Romagnani, L., Brenner, C.M., Green, J.S., Gallegos, P., Streeter, M.J.V., Carroll, D.C., Tresca, O., Dover, N., Palmer, C.A.J., Schreiber, J., Neely, D., Najmudin, Z., McKenna, P., Zepf, M., \& Borghesi, M. 2010. Calibration of Thomson 
parabola-MCP assembly for multi-MeV ion spectroscopy. Nuclear Instruments and Methods in Physics Research Section A: Accelerators, Spectrometers, Detectors and Associated Equipment, 623(2), 712 - 715. 1rs International Conference on Frontiers in Diagnostics Technologies. 65

[Psikal et al., 2008] Psikal, J., Tikhonchuk, V. T., Limpouch, J., Andreev, A. A., \& Brantov, A. V. 2008. Ion acceleration by femtosecond laser pulses in small multispecies targets. Physics of Plasmas, 15(5), 053102. 53

[Quesnel \& Mora, 1998] Quesnel, Brice, \& Mora, Patrick. 1998. Theory and simulation of the interaction of ultraintense laser pulses with electrons in vacuum. Phys. Rev. E, 58(Sep), 3719-3732. 31

[Reynolds et al., 1950] Reynolds, G. T., Harrison, F. B., \& Salvini, G. 1950. Liquid Scintillation Counters. Phys. Rev., 78(May), 488-488. 68

[Robinson et al., 2008] Robinson, A. P. L., Sherlock, M., \& Norreys, P. A. 2008. Artificial Collimation of Fast-Electron Beams with Two Laser Pulses. Phys. Rev. Lett., 100(Jan), 025002. 44

[Robson et al., 2007] Robson, L, Simpson, PT, McKenna, P, Ledingham, KWD, Clarke, RJ, McCanny, T, Neely, D, Lundh, Olle, Lindau, Filip, Wahlström, ClaesGöran, \& Zepf, M. 2007. Scaling of proton acceleration driven by petawatt-laserplasma interactions. Nature Physics, 3(58), 58-62. 54

[Rohrlich \& Carlson, 1954] Rohrlich, F., \& Carlson, B. C. 1954. Positron-Electron Differences in Energy Loss and Multiple Scattering. Phys. Rev., 93(Jan), 38-44. 40

[Romagnani et al., 2005] Romagnani, L., Fuchs, J., Borghesi, M., Antici, P., Audebert, P., Ceccherini, F., Cowan, T., Grismayer, T., Kar, S., Macchi, A., Mora, P., Pretzler, G., Schiavi, A., Toncian, T., \& Willi, O. 2005. Dynamics of Electric Fields Driving the Laser Acceleration of Multi-MeV Protons. Phys. Rev. Lett., 95(Oct), 195001. 24, 50

[Rossi \& Greisen, 1941] Rossi, Bruno, \& Greisen, Kenneth. 1941. Cosmic-Ray Theory. Rev. Mod. Phys., 13(Oct), 240-309. 57

[Roth et al., 2001] Roth, M., Cowan, T. E., Key, M. H., Hatchett, S. P., Brown, C., Fountain, W., Johnson, J., Pennington, D. M., Snavely, R. A., Wilks, S. C., Yasuike, K., Ruhl, H., Pegoraro, F., Bulanov, S. V., Campbell, E. M., Perry, M. D., \& Powell, H. 2001. Fast Ignition by Intense Laser-Accelerated Proton Beams. Phys. Rev. Lett., 86(Jan), 436-439. 2, 24

[Roth et al., 2013] Roth, M., Jung, D., Falk, K., Guler, N., Deppert, O., Devlin, M., Favalli, A., Fernandez, J., Gautier, D., Geissel, M., Haight, R., Hamilton, C. E., Hegelich, B. M., Johnson, R. P., Merrill, F., Schaumann, G., Schoenberg, 
K., Schollmeier, M., Shimada, T., Taddeucci, T., Tybo, J. L., Wagner, F., Wender, S. A., Wilde, C. H., \& Wurden, G. A. 2013. Bright Laser-Driven Neutron Source Based on the Relativistic Transparency of Solids. Phys. Rev. Lett., 110(Jan), 044802. 46

[Rozmus et al., 1996] Rozmus, W., Tikhonchuk, V. T., \& Cauble, R. 1996. A model of ultrashort laser pulse absorption in solid targets. Physics of Plasmas, 3(1), 360-367. 32

[Rutherford, 1911] Rutherford, E. 1911. The scattering of $a$ and $b$ particles by matter and the structure of the atom. Philosophical Magazine, 92(4), 379-398. 38, 67

[Saint-Gobain-Crystals, 2020] Saint-Gobain-Crystals. 2020. Website: https:// www. crystals. saint-gobain. com/. $71,82,83,90$

[Santala et al., 2001] Santala, M. I. K., Zepf, M., Beg, F. N., Clark, E. L., Dangor, A. E., Krushelnick, K., Tatarakis, M., Watts, I., Ledingham, K. W. D., McCanny, T., Spencer, I., Machacek, A. C., Allott, R., Clarke, R. J., \& Norreys, P. A. 2001. Production of radioactive nuclides by energetic protons generated from intense laser-plasma interactions. Applied Physics Letters, 78(1), 19-21. 2, 24

[Sawada et al., 2014] Sawada, H, Wei, MS, Chawla, S, Morace, A, Akli, K, Yabuuchi, T, Nakanii, N, Key, MH, Patel, PK, Mackinnon, AJ, McLean, HS, Stephens, RB, \& Beg, FN. 2014. Investigation of fast-electron-induced K x rays in laser-produced blow-off plasma. Physical review. E, Statistical, nonlinear, and soft matter physics, 89(3), 033105. 36, 109

[Schawlow \& Townes, 1958] Schawlow, A. L., \& Townes, C. H. 1958. Infrared and Optical Masers. Phys. Rev., 112(Dec), 1940-1949. 8

[Schollmeier et al., 2014] Schollmeier, M., Geissel, M., Sefkow, A. B., \& Flippo, K. A. 2014. Improved spectral data unfolding for radiochromic film imaging spectroscopy of laser-accelerated proton beams. Review of Scientific Instruments, 85(4), 043305. 65

[Schorr \& Torney, 1950] Schorr, M. G., \& Torney, F. L. 1950. Solid Non-Crystalline Scintillation Phosphors. Physical Review, 80(9), 474-474. 68

[Schreiber et al., 2006] Schreiber, J., Bell, F., Grüner, F., Schramm, U., Geissler, M., Schnürer, M., Ter-Avetisyan, S., Hegelich, B. M., Cobble, J., Brambrink, E., Fuchs, J., Audebert, P., \& Habs, D. 2006. Analytical Model for Ion Acceleration by High-Intensity Laser Pulses. Phys. Rev. Lett., 97(Jul), 045005. 45, 47, 49, 55, 122

[Scott et al., 2012] Scott, R. H. H., Beaucourt, C., Schlenvoigt, H.-P., Markey, K., Lancaster, K. L., Ridgers, C. P., Brenner, C. M., Pasley, J., Gray, R. J., Musgrave, I. O., Robinson, A. P. L, Li, K., Notley, M. M., Davies, J. R., Baton, S. D., Santos, 
J. J., Feugeas, J.-L., Nicolaï, Ph., Malka, G., Tikhonchuk, V. T., McKenna, P., Neely, D., Rose, S. J., \& Norreys, P. A. 2012. Controlling Fast-Electron-Beam Divergence Using Two Laser Pulses. Phys. Rev. Lett., 109(Jul), 015001. 44

[Scott, 1963] Scott, William T. 1963. The Theory of Small-Angle Multiple Scattering of Fast Charged Particles. Rev. Mod. Phys., 35(Apr), 231-313. 57

[Scullion et al., 2016] Scullion, C., Doria, D., Romagnani, L., Ahmed, H., Alejo, A., Ettlinger, O.C., Gray, R.J., Green, J., Hicks, G.S., Jung, D., Naughton, K., Padda, H., Poder, K., Scott, G.G., Symes, D.R., Kar, S., McKenna, P., Najmudin, Z., Neely, D., Zepf, M., \& Borghesi, M. 2016. Angularly resolved characterization of ion beams from laser-ultrathin foil interactions. Journal of Instrumentation, 11(09), C09020-C09020. 65

[Seltzer, 1974] Seltzer, S M. 1974. Transmission of electrons through foils. Nuclear Instruments and Methods, 119(4). 40

[Shikhaliev et al., 2004] Shikhaliev, Polad M., Xu, Tong, Le, Huy, \& Molloi, Sabee. 2004. Scanning-slit photon counting x-ray imaging system using a microchannel plate detector. Medical Physics, 31(5), 1061-1071. 60

[Sinenian et al., 2011] Sinenian, N., Rosenberg, M. J., Manuel, M., McDuffee, S. C., Casey, D. T., Zylstra, A. B., Rinderknecht, H. G., Gatu Johnson, M., Sguin, F. H., Frenje, J. A., Li, C. K., \& Petrasso, R. D. 2011. The response of CR-39 nuclear track detector to $19 \mathrm{MeV}$ protons. Review of Scientific Instruments, 82(10), 103303. 65

[Smith et al., 1968] Smith, D.L., Polk, R.G., \& Miller, T.G. 1968. Measurement of the response of several organic scintillators to electrons, protons and deuterons. Nuclear Instruments and Methods, 64(2), 157-166. 68

[Snavely et al., 2000] Snavely, R. A., Key, M. H., Hatchett, S. P., Cowan, T. E., Roth, M., Phillips, T. W., Stoyer, M. A., Henry, E. A., Sangster, T. C., Singh, M. S., Wilks, S. C., MacKinnon, A., Offenberger, A., Pennington, D. M., Yasuike, K., Langdon, A. B., Lasinski, B. F., Johnson, J., Perry, M. D., \& Campbell, E. M. 2000. Intense High-Energy Proton Beams from Petawatt-Laser Irradiation of Solids. Phys. Rev. Lett., 85(Oct), 2945-2948. 1, 23, 24, 45, 49, 50, 65

[Solodov \& Betti, 2008] Solodov, A. A., \& Betti, R. 2008. Stopping power and range of energetic electrons in dense plasmas of fast-ignition fusion targets. Physics of Plasmas, 15(4), 042707. 42

[Spencer et al., 2003] Spencer, I., Ledingham, K. W. D., McKenna, P., McCanny, T., Singhal, R. P., Foster, P. S., Neely, D., Langley, A. J., Divall, E. J., Hooker, C. J., Clarke, R. J., Norreys, P. A., Clark, E. L., Krushelnick, K., \& Davies, J. R. 2003. Experimental study of proton emission from 60-fs, 200-mJ high-repetitionrate tabletop-laser pulses interacting with solid targets. Phys. Rev. E, 67(Apr), 046402. 51 
[Stephens et al., 2004] Stephens, R. B., Snavely, R. A., Aglitskiy, Y., Amiranoff, F., Andersen, C., Batani, D., Baton, S. D., Cowan, T., Freeman, R. R., Hall, T., Hatchett, S. P., Hill, J. M., Key, M. H., King, J. A., Koch, J. A., Koenig, M., MacKinnon, A. J., Lancaster, K. L., Martinolli, E., Norreys, P., Perelli-Cippo, E., Rabec Le Gloahec, M., Rousseaux, C., Santos, J. J., \& Scianitti, F. 2004. $K_{\alpha}$ fluorescence measurement of relativistic electron transport in the context of fast ignition. Phys. Rev. E, 69(Jun), 066414. 123

[Sternheimer, 1952] Sternheimer, R. M. 1952. The Density Effect for the Ionization Loss in Various Materials. Phys. Rev., 88(Nov), 851-859. 57

[Sternheimer, 1966] Sternheimer, R. M. 1966. Density Effect for the Ionization Loss of Charged Particles. Phys. Rev., 145(May), 247-250. 40

[Sternheimer et al., 1982] Sternheimer, R. M., Seltzer, S. M., \& Berger, M. J. 1982. Density effect for the ionization loss of charged particles in various substances. Phys. Rev. B, 26(Dec), 6067-6076. 57

[Strickland \& Mourou, 1985] Strickland, Donna, \& Mourou, Gerard. 1985. Compression of amplified chirped optical pulses. Optics Communications, 56(3), $219-221$. $1,7,12,26$

[Tajima \& Dawson, 1979] Tajima, T., \& Dawson, J. M. 1979. Laser Electron Accelerator. Phys. Rev. Lett., 43(Jul), 267-270. 21

[Taylor et al., 1951] Taylor, C. J., Jentschke, W. K., Remley, M. E., Eby, F. S., \& Kruger, P. G. 1951. Response of Some Scintillation Crystals to Charged Particles. Phys. Rev., 84(Dec), 1034-1043. 81

[Temporal et al., 2002] Temporal, M., Honrubia, J. J., \& Atzeni, S. 2002. Numerical study of fast ignition of ablatively imploded deuteriumtritium fusion capsules by ultra-intense proton beams. Physics of Plasmas, 9(7), 3098-3107. 24

[Thomson, 1913] Thomson, J.J. 1913. Bakerian lecture: Rays of positive electricity. Proceedings of the Royal Society of London. Series A, 89(607), . 120. 62

[Torrisi, 1997] Torrisi, L. 1997. Radiation damage in polyvinyltoluene (PVT) induced by $50400 \mathrm{keV}$ helium beams. Radiation Effects and Defects in Solids, 143(1), 19-31. 90

[Torrisi, 2000] Torrisi, L. 2000. Plastic scintillator investigations for relative dosimetry in proton-therapy. Nuclear Instruments and Methods in Physics Research Section B: Beam Interactions with Materials and Atoms, 170(3), 523-530. 89

[Town et al., 2005] Town, R.P.J., Chen, C., Cottrill, L.A., Key, M.H., Kruer, W.L., Langdon, A.B., Lasinski, B.F., Snavely, R.A., Still, C.H., Tabak, M., Welch, D.R., \& Wilks, S.C. 2005. Simulations of electron transport for fast ignition using LSP. 
Nuclear Instruments and Methods in Physics Research Section A: Accelerators, Spectrometers, Detectors and Associated Equipment, 544(1), 61 - 66. Proceedings of the 15th International Symposium on Heavy Ion Inertial Fusion. 36

[Tsai, 1974] Tsai, Yung-Su. 1974. Pair production and bremsstrahlung of charged leptons. Rev. Mod. Phys., 46(Oct), 815-851. 58

[Varelas \& Biersack, 1970] Varelas, C., \& Biersack, J. 1970. Reflection of energetic particles from atomic or ionic chains in single crystals. Nuclear Instruments and Methods, 79(2), 213 - 218. 57

[Volpe et al., 2011] Volpe, L., Batani, D., Vauzour, B., Nicolai, Ph., Santos, J. J., Regan, C., Morace, A., Dorchies, F., Fourment, C., Hulin, S., Perez, F., Baton, S., Lancaster, K., Galimberti, M., Heathcote, R., Tolley, M., Spindloe, Ch., Koester, P., Labate, L., Gizzi, L. A., Benedetti, C., Sgattoni, A., Richetta, M., Pasley, J., Beg, F., Chawla, S., Higginson, D. P., \& MacPhee, A. G. 2011. Proton radiography of laser-driven imploding target in cylindrical geometry. Physics of Plasmas, 18(1), 012704. 24

[Volpe et al., 2013] Volpe, L., Batani, D., Birindelli, G., Morace, A., Carpeggiani, P., Xu, M. H., Liu, F., Zhang, Y., Zhang, Z., Lin, X. X., Liu, F., Wang, S. J., Zhu, P. F., Meng, L. M., Wang, Z. H., Li, Y. T., Sheng, Z. M., Wei, Z. Y., Zhang, J., Santos, J. J., \& Spindloe, C. 2013. Propagation of a short-pulse laser-driven electron beam in matter. Physics of Plasmas, 20(3), 033105. 45

[Volpe et al., 2014] Volpe, L., Feugeas, J-L., Nicolai, Ph., Santos, J. J., Touati, M., Breil, J., Batani, D., \& Tikhonchuk, V. 2014. Controlling the fast electron divergence in a solid target with multiple laser pulses. Phys. Rev. E, 90(Dec), 063108. 44

[Volpe et al., 2019] Volpe, L., Fedosejevs, R., G. Gatti, G., Pérez-Hernández, J A, Méndez, C., Apiñaniz, J., Vaisseau, X., Salgado, C., Huault, M., Malko, S., Zeraouli, G., Ospina, V., Longman, A., De Luis, D., Li, K., Varela, O., García, E., Hernández, I., Pisonero, J.D., García Ajates, J., Alvarez, J.M, García, C., Rico, M., Arana, D., Hernández-Toro, J., \& Roso, L. 2019. Generation of high energy laser-driven electron and proton sources with the 200 TW system VEGA 2 at the Centro de Laseres Pulsados. High Power Laser Science and Engineering, 7, e25. 3

[Wagner et al., 2016] Wagner, F., Deppert, O., Brabetz, C., Fiala, P., Kleinschmidt, A., Poth, P., Schanz, V. A., Tebartz, A., Zielbauer, B., Roth, M., Stöhlker, T., \& Bagnoud, V. 2016. Maximum Proton Energy above $85 \mathrm{MeV}$ from the Relativistic Interaction of Laser Pulses with Micrometer Thick $\mathrm{CH}_{2}$ Targets. Phys. Rev. Lett., 116(May), 205002. 2, 54

[Walske, 1952] Walske, M. C. 1952. The Stopping Power of K-Electrons. Phys. Rev., 88(Dec), 1283-1289. 57 
[Wilks et al., 1992] Wilks, S. C., Kruer, W. L., Tabak, M., \& Langdon, A. B. 1992. Absorption of ultra-intense laser pulses. Phys. Rev. Lett., 69(Aug), 1383-1386. 35, 36

[Wilks et al., 2001] Wilks, S. C., Langdon, A. B., Cowan, T. E., Roth, M., Singh, M., Hatchett, S., Key, M. H., Pennington, D., MacKinnon, A., \& Snavely, R. A. 2001. Energetic proton generation in ultra-intense lasersolid interactions. Physics of Plasmas, 8(2), 542-549. 1, 45

[Wolff \& Stephens, 1953] Wolff, M. M., \& Stephens, W. E. 1953. A Pulsed Mass Spectrometer with Time Dispersion. Review of Scientific Instruments, 24(8), 616617. 65

[Woodward, 1948] Woodward, P.M. 1948. The theoretical precision with which an arbitrary radiation-pattern may be obtained from a source of finite size. Journal of the Institution of Electrical Engineers - Part III: Radio and Communication Engineering, 95(September), 363-370(7). 29

[Woryna et al., 1996] Woryna, E., Parys, P., Woowski, J., \& Mrz, W. 1996. Corpuscular diagnostics and processing methods applied in investigations of laser-produced plasma as a source of highly ionized ions. Laser and Particle Beams, 14(3), 293321. 58

[Zeil et al., 2010] Zeil, K, Kraft, S D, Bock, S, Bussmann, M, Cowan, T E, Kluge, T, Metzkes, J, Richter, T, Sauerbrey, R, \& Schramm, U. 2010. The scaling of proton energies in ultrashort pulse laser plasma acceleration. New Journal of Physics, 12(4), 045015. 54, 55

[Zeraouli et al., 2019] Zeraouli, G., Gatti, G., Longman, A., Pérez-Hernández, J. A., Arana, D., Batani, D., Jakubowska, K., Volpe, L., Roso, L., \& Fedosejevs, R. 2019. Development of an adjustable Kirkpatrick-Baez microscope for laser driven x-ray sources. Review of Scientific Instruments, 90(6), 063704. 3

[Zeraouli, 2020] Zeraouli, Ghassan. 2020. Experimental studies for generation, transport and applications of ultra-fast laser driven X-ray sources. Theses, University of Salamanca. 21 